\title{
Extracellular vesicle release of $\alpha$-Synuclein is mediated by SUMOylation
}

\author{
Dissertation \\ for the award of the degree \\ "Doctor rerum naturalium" \\ of the Georg-August-Universität Göttingen
}

within the Program Molecular Physiology of the Brain

of the Georg-August University School of Science (GAUSS)

\author{
submitted by \\ Marcel Kunadt \\ from Eisenhüttenstadt
}

Göttingen, 2015 


\section{Members of Thesis Committee}

\section{Prof. Dr. Mikael Simons}

Dept. of Cellular Neuroscience, MPI for Experimental Medicine

Prof. Dr. André Fischer

Dept. of Psychiatry and Psychotherapy, University Medical Center Göttingen, DZNE Göttingen

\section{Prof. Dr. Silvio Rizzoli}

Dept. of Neuro-and Sensory Physiology, University Medical Center Göttingen

\section{Members of the Examination Board}

Prof. Dr. Dr. Hannelore Ehrenreich

Dept. of Clinical Neuroscience, MPI for Experimental Medicine

\section{Prof. Dr. Tiago Fleming Outeiro}

Dept. of Neurodegeneration and Restorative Research, University Medical Center Göttingen

\section{Prof. Dr. Alexander Flügel}

Institute for Multiple Sclerosis Research, University Medical Center Göttingen, Dept. of Neuroimmunology 


\section{Affirmation}

I hereby declare that this thesis was written independently and with no other sources and aids than quoted.

Göttingen, 08.05.2015

Marcel Kunadt 


\section{Acknowledgements - Danksagung}

Mein besonderer Dank gilt in erster Linie meiner Doktormutter Frau Prof. Dr. Anja Schneider, für das mir entgegengebrachte Vertrauen, für ihre exzellente Betreuung dieser Doktorarbeit, für die aktuelle und interessante Themenstellung, ihre wissenschaftlichen Ratschläge und Anregungen, sowie ihr aufrichtiges Interesse am Fortgang dieser Arbeit. Ein weiterer Dank geht an Thomas M. Jovin für seine großes Engagement bei der Analyse der SUMOLipidbindungsstudien und seine stets fruchtvollen Ratschläge bei der Verbesserung dieser neuen Messmethode.

Weiterhin gebührt mein Dank den Mitgliedern meines „Thesis Committees“, Prof. Dr. Mikael Simons, Prof. Dr. André Fischer und Prof. Dr. Silvio Rizzoli, für die Unterstützung und die stets fruchtvollen Diskussionen und Kommentare, die zum Abschluss dieser Arbeit beigetragen haben. Ein weiterer Dank gilt den Mitgliedern meines erweiterten Prüfungsausschusses, Prof. Dr. Dr. Hannelore Ehrenreich, Prof. Dr. Tiago Fleming Outeiro, sowie Prof. Dr. Alexander Flügel.

Einen speziellen Dank möchte ich an die vielen Kollaborationspartner richten, die maßgeblich zum Erfolg dieser Arbeit beigetragen haben. Für die finanzielle Unterstützung Danke ich der DFG (Deutsche Forschungsgemeinschaft), sowie dem DFG Research Center Nanoscale Microscopy and Molecular Physiology of the Brain (CNMPB).

Danke sagen möchte ich auch an alle derzeitigen und ehemaligen Mitglieder der AG Schneider. Ich danke euch allen für das wundervolle Arbeitsklima, die Mittagspausen in der Sonne, oder einfach mal für das „Käffchen“ nebenbei. Weiterhin danke ich euch für die große Unterstützung in der letzten Zügen dieser Arbeit. Danke, dass ihr immer bemüht wart mich aufzumuntern und immer ein offenes Ohr für mich hattet. Ein spezieller Dank gilt vor allem Anne, Belisa und Beate für die Korrekturen dieser Arbeit. Petra Wilken gebührt ein weiterer Dank für die vielen lustigen Stunden auch außerhalb des Labors. Aus der AG Simons danke ich Tina Kling, für die tollen Gespräche und für deinen Benni, der mich immer ein wenig aufgebaut und im höchsten Maße zum Lachen gebracht hat.

Anne dir gilt mein besonderer Dank. Zuerst möchte ich dir danken, dass es dich überhaupt gibt und dass du trotz diverser Macken immer zu mir gehalten hast und mich so akzeptierst wie ich bin. Ich weiß die letzte Zeit war nicht einfach für uns beide. Du hast mich in der ganzen Zeit immer wieder aufgebaut und warst auch in schwierigen Situationen immer für mich da und hast an mich geglaubt. 
Ich danke dir von ganzem Herzen für deine scheinbar unerschöpfliche Kraft, sowie deine seelische und moralische Unterstützung beim Anfertigen dieser Arbeit. Danke Anne für alles.

Meine „Schergen“ haben ebenfalls einen besonderen Dank verdient. Ich danke euch für die gemeinsame Zeit und all den Spaß den wir zusammen erlebt haben. Ingo dir gebührt ein besonderer Dank. Du bist der beste Mitbewohner und Freund den Man(n) sich wünschen kann. Ich danke dir dafür, dass du immer da warst, mich immer wieder aufgerichtet hast und immer ein offenes Ohr für mich hattest. An all die anderen Verrückten, danke für eure Freundschaft. Danke an Boscabana und seine anderen Kollegen.

Zum Schluss geht ein ganz besonderer Dank an meine ganze Familie. Insbesondere möchte ich meinen Eltern Viola und Andreas danken, sowie dir Oma Inge. Ich danke euch, dass ihr mir das Studium ermöglicht habt, dass ihr immer für mich da wart wenn ich euch gebraucht habe und immer ein offenes Ohr für mich hattet und versucht habt, auch wenn es nicht immer leicht war, mich zum weiter machen zu motivieren. Auch dir Linda möchte ich danken, danke, dass ich dich als Schwester habe und auch wenn wir uns selten sehen, es immer schön ist wenn wir uns mal treffen und ein wenig Zeit zusammen haben.

Zum Schluss noch ein Dank an dich Opa. Leider hast du es nicht mehr geschafft so lange zu kämpfen, um den Abschluss meiner Doktorarbeit zu erleben. Ich weiß, dass du voller Stolz von oben herab schaust und mitbekommen wirst, dass ich es geschafft habe. Danke Opa für alles was du mir beigebracht hast, dir sei diese Arbeit gewidmet. 


\section{Table of Contents}

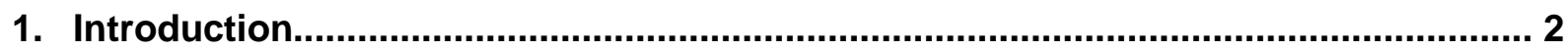

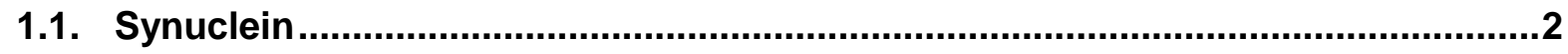

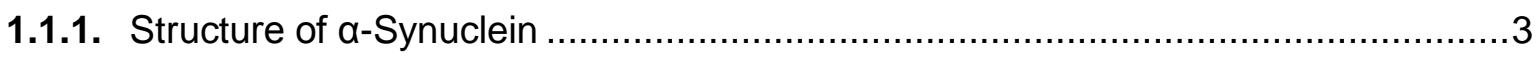

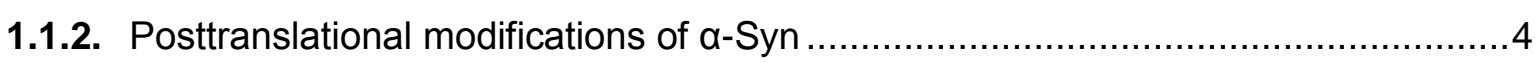

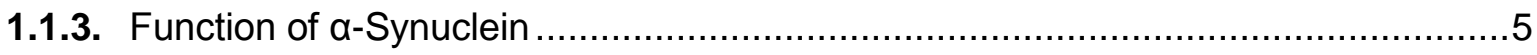

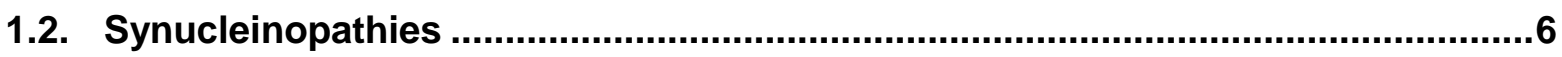

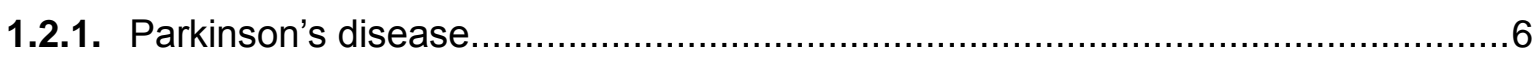

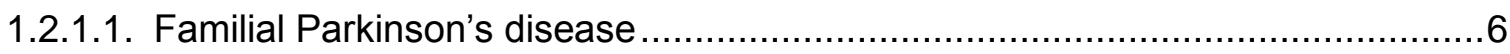

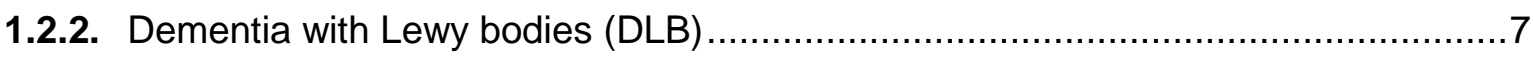

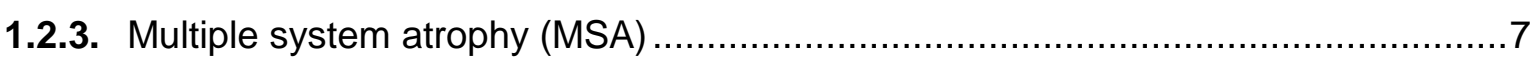

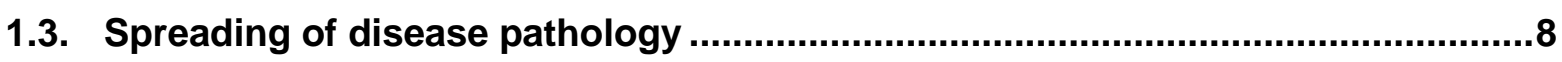

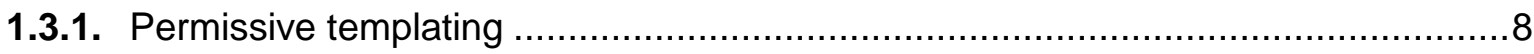

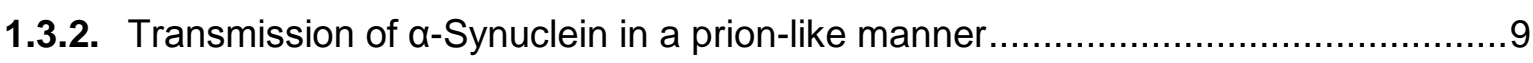

1.4. Extracellular vesicles.................................................................................11

1.4.1. Morphology and composition of extracellular vesicles ....................................11

1.4.2. Biogenesis of extracellular vesicles ............................................................ 14

1.4.3. Secretion of extracellular vesicles............................................................. 16

1.4.4. Function of extracellular vesicles ................................................................... 17

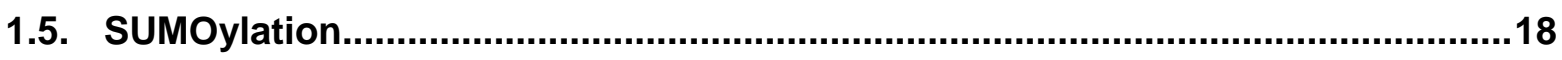

1.5.1. The family of small ubiquitin like modifiers SUMO ........................................... 18

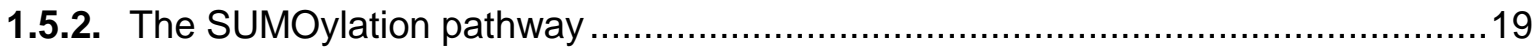

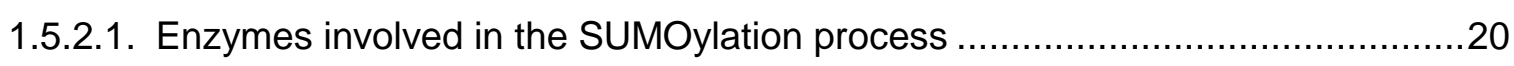

1.5.2.2. Non covalent SUMO binding mediated by SIM .........................................21

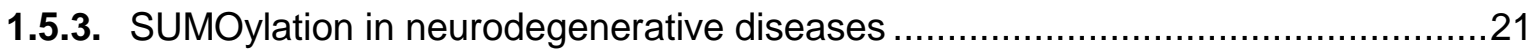

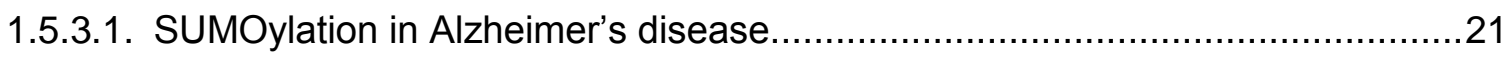

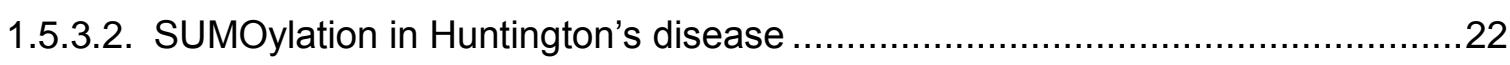

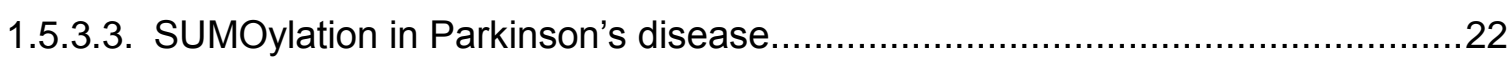

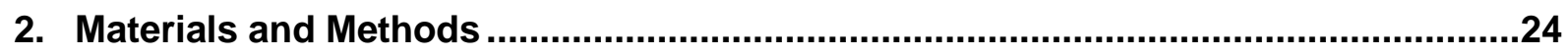

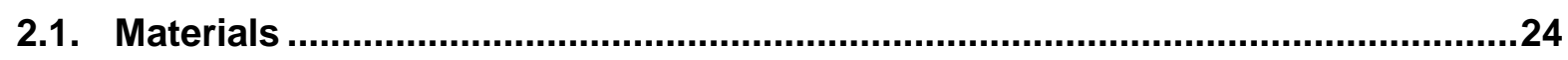

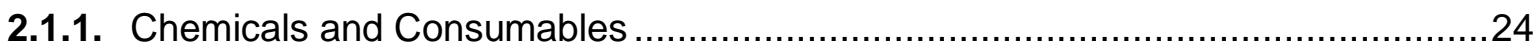

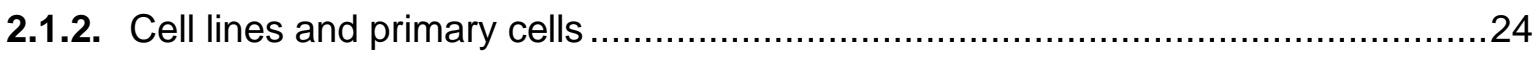

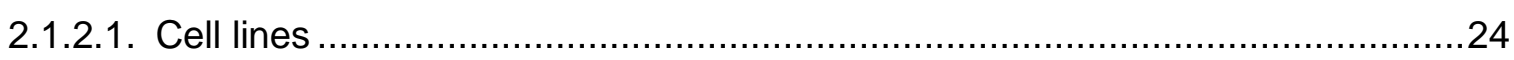

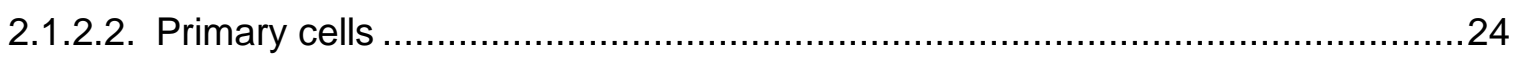

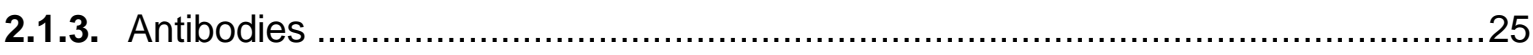

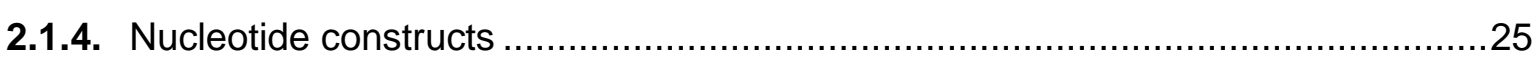

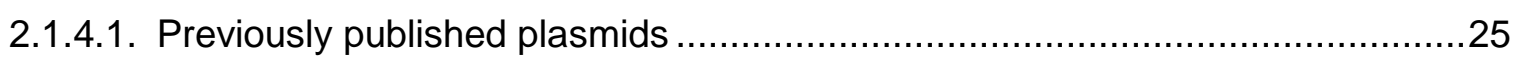

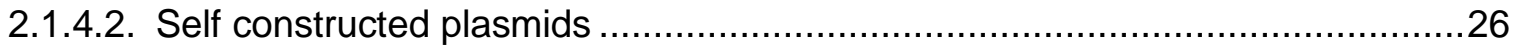

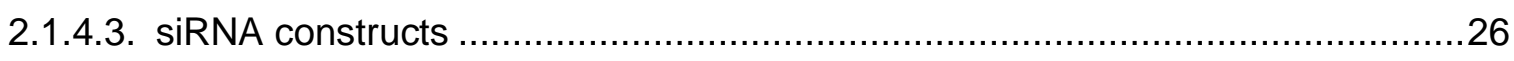




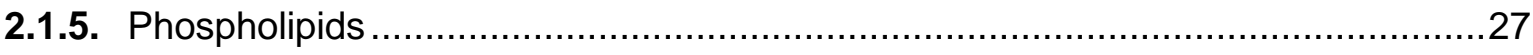

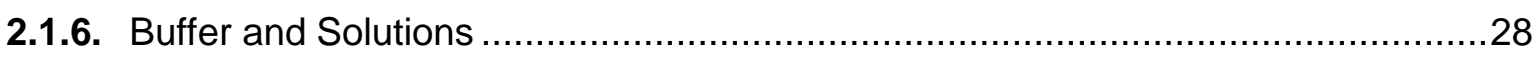

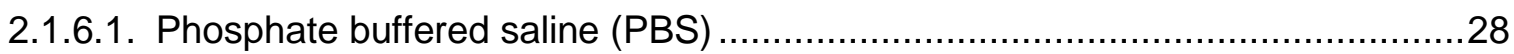

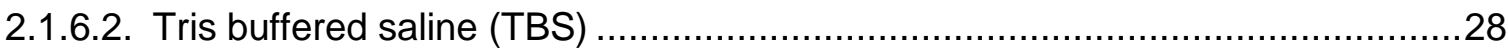

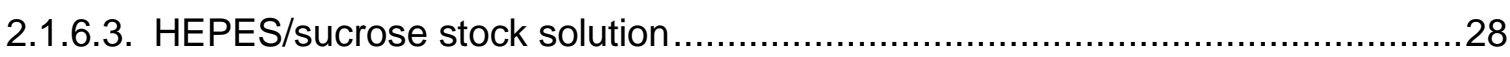

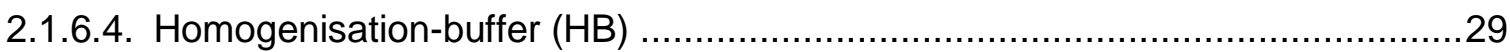

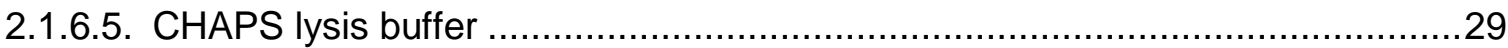

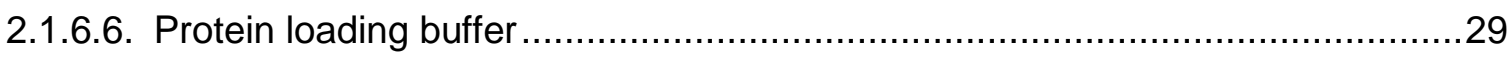

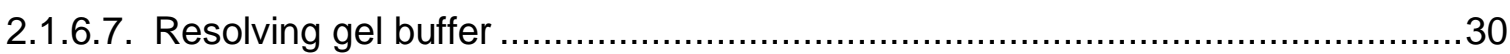

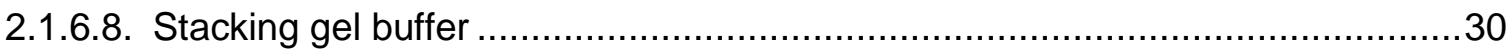

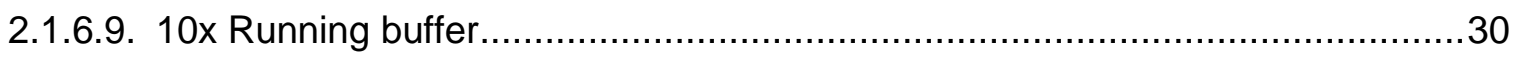

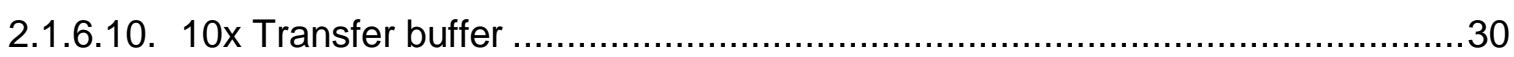

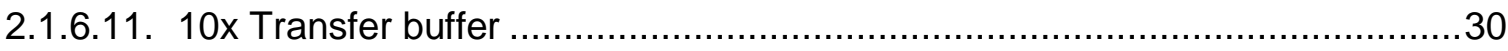

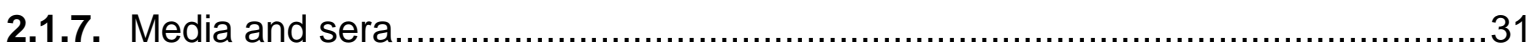

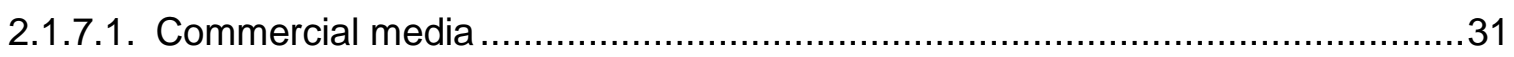

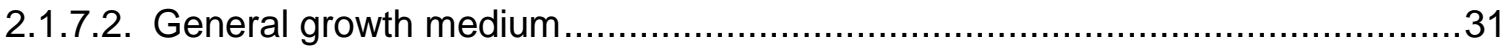

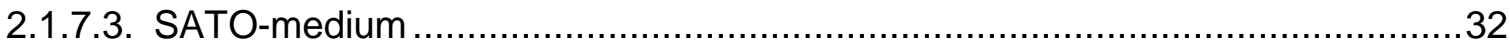

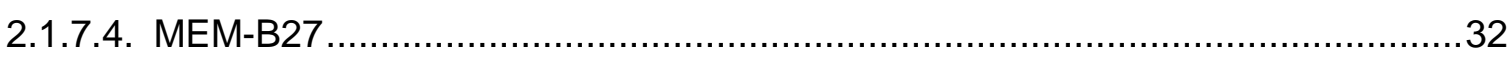

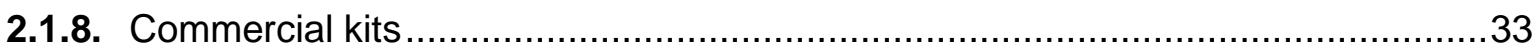

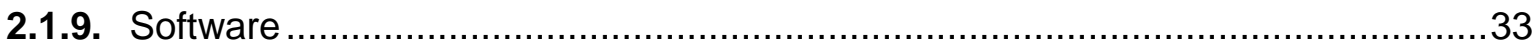

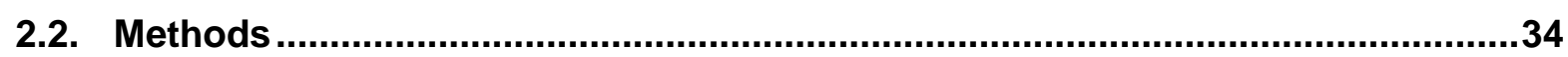

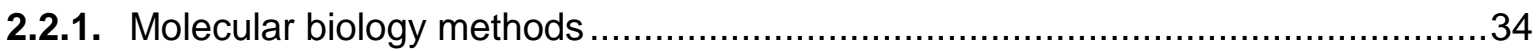

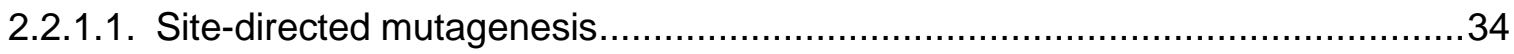

2.2.1.2. Transformation of Escherichia coli (E. coli) .................................................. 34

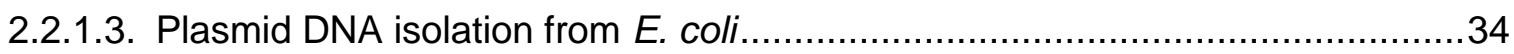

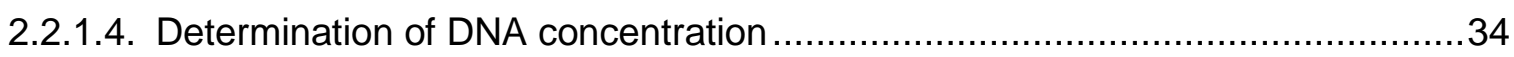

2.2.1.5. Expression and purification of sumoylated $\alpha$-Synuclein.....................................35

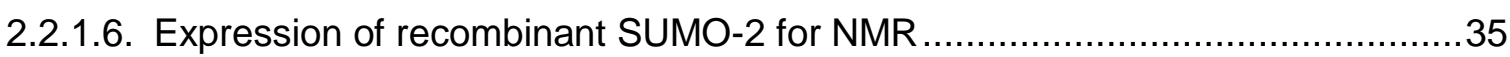

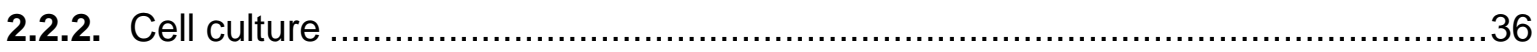

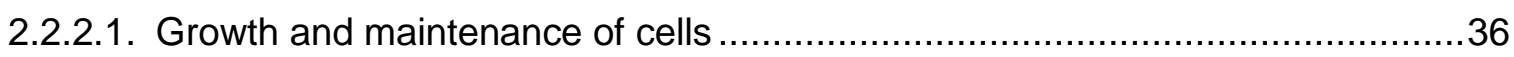

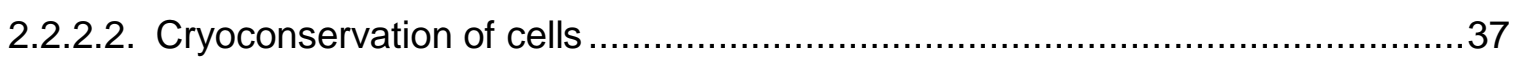

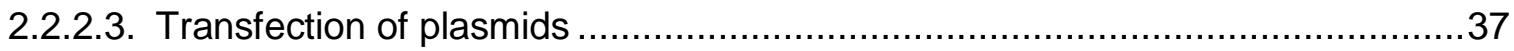

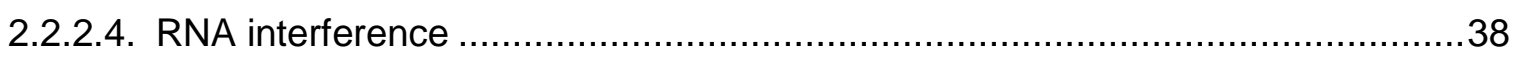

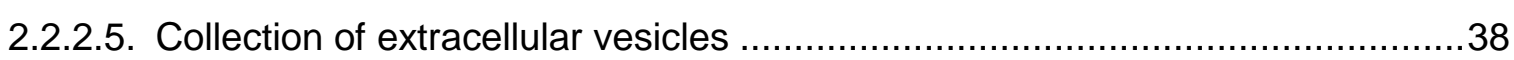

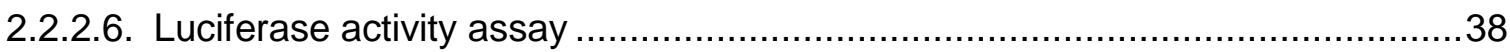

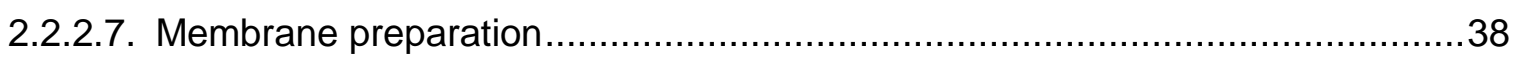

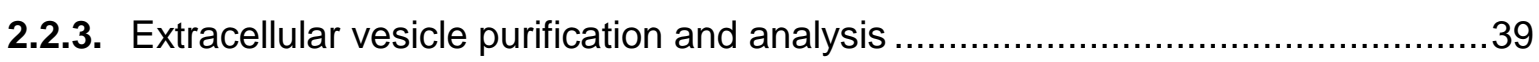

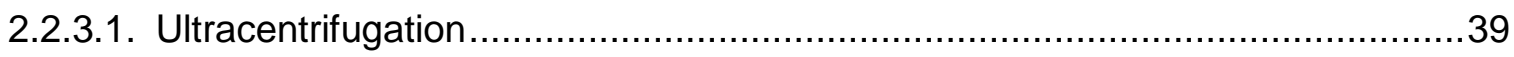

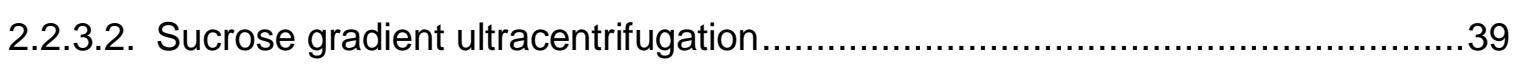

2.2.3.3. Nanoparticle tracking analysis (NTA) ................................................... 40 


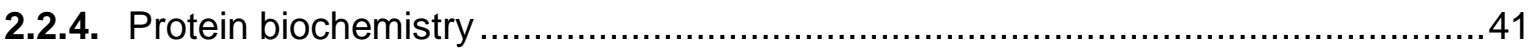

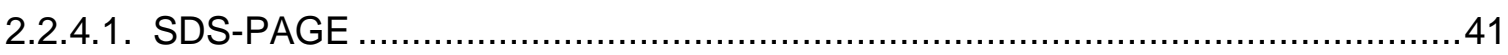

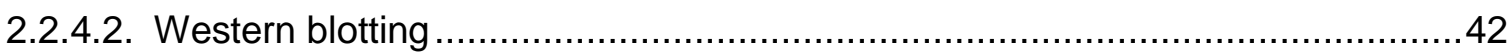

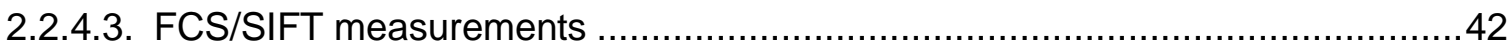

2.2.4.4. Electrochemiluminescence assay for quantification of $\alpha$-Synuclein ..................43

2.2.4.5. Labelling of SUMO-2 with the ESPIT dye MFM............................................43

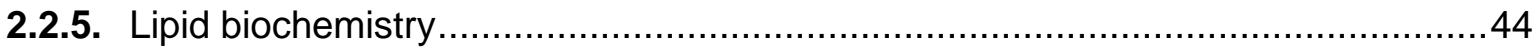

2.2.5.1. Preparation of Small Unilamellar Vesicles (SUVs) ......................................44

2.2.5.2. Membrane binding assay of SUMO-2 ...................................................... 44

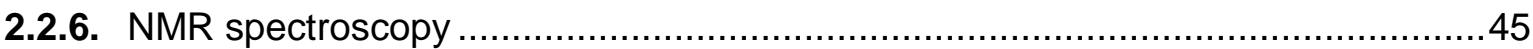

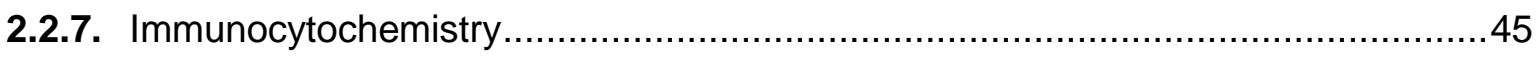

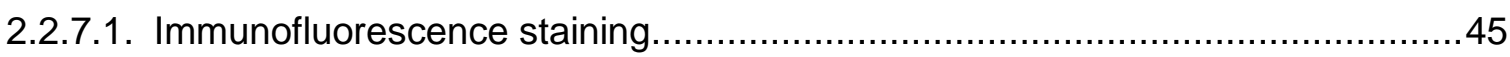

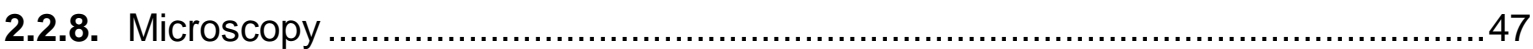

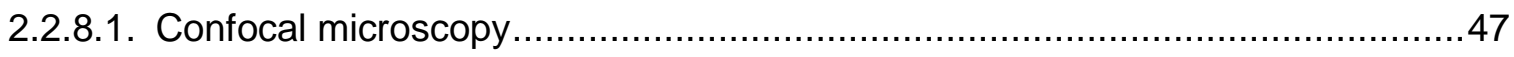

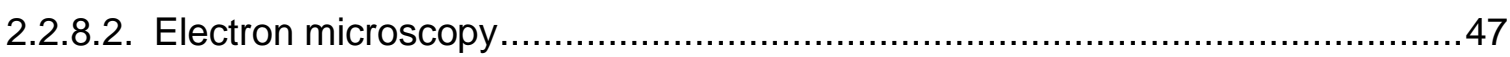

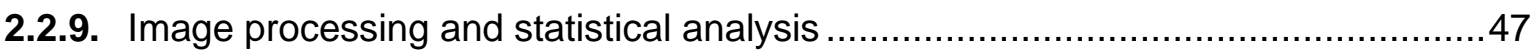

2.2.9.1. Quantification of extracellular vesicle secretion .......................................... 47

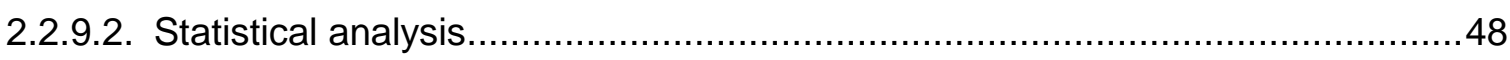

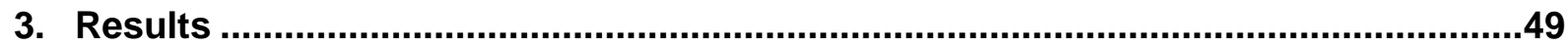

3.1. $\alpha$-Synuclein is released in extracellular vesicles............................................... 49

3.1.1. $\alpha$-Synuclein is released in extracellular vesicles derived from N2a cells ...............49

3.1.2. $\alpha$-Synuclein is localized in extracellular vesicles in vivo .....................................51

3.1.3. $\alpha$-Synuclein is predominantly localized in the lumen of EVs.............................53

3.2. The extracellular release of $\alpha$-Synuclein is regulated by membrane binding.....56

3.3. SUMOylation regulates membrane binding and extracellular vesicle release

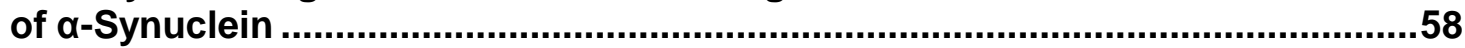

3.3.1. SUMOylation modulates membrane binding of $\alpha$-Synuclein ..............................58

3.3.2. Extracellular vesicle release of $\alpha$-Synuclein is regulated by SUMOylation .............62

3.3.2.1. Silencing of Ubc9 decreases the release of $\alpha$-Synuclein within extracellular vesicles.

3.3.2.2. $\alpha$-Synuclein fusion with SUMO-2 increases the release of $\alpha$-Synuclein within extracellular vesicles

3.3.2.3. Co-expression of SUMO-2 increases the release of $\alpha$-Synuclein within extracellular vesicles

3.3.2.4. Isopeptidase acitivity in extracellular vesicles results in a rapid deconjugation of SUMO.

\subsection{SUMOylation can act as sorting signal for the release within extracellular} vesicles.

3.4.1. SUMO-2 targets the cytosolic protein GFP to extracellular vesicle release. .76

3.4.2. SUMO-1 also modulates extracellular vesicle sorting of the cytosolic protein GFP

3.4.3. SUMOylation increases the extracellular vesicle release of the transmembrane protein amyloid precursor protein (APP)... 
3.5. Extracellular vesicle release of SUMO-2 is ESCRT-dependent.

3.5.1. RNA Interference with the ESCRT components Alix and Tsg101 decrease extracellular vesicle release of a SUMO-2-GFP fusion protein

3.5.2. Co-expression of the dominant negative mutant of VPS4 decreases the extracellular vesicles release of a GFP-SUMO-2 fusion protein.

3.5.3. Extracellular vesicle release of SUMO-2 does not depend on the canonical SUMO protein interaction motif Q30 F31 I33 .................................................87

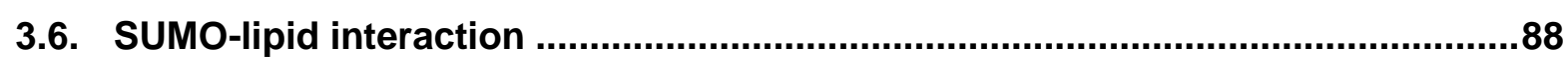

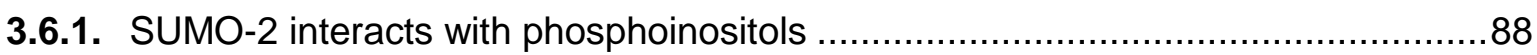

3.7. Identification of the membrane interaction motif of SUMO-2 .............................90

3.7.1. The membrane interaction motif of SUMO-2 is localised to the hydrophobic cleft and nearby loops

3.7.1.1. Mutations in the hydrophobic cleft and N-terminally loop domains of SUMO-2 decreases the membrane binding propensity of SUMO-2 ................92

3.7.1.2. Mutations in the hydrophobic cleft and N-terminally loop domains of SUMO-2 decreases sorting into extracellular vesicles.

3.8. Co-expression of the dominant negative mutant of VPS4 decreases extracellular vesicle release of $\alpha$-Synuclein.

3.9. Inhibition of endosome maturation by overexpression of dominant negative Rab5 does not trap $\alpha$-Syn or SUMO-2 in enlarged intraluminal vesicles .............96

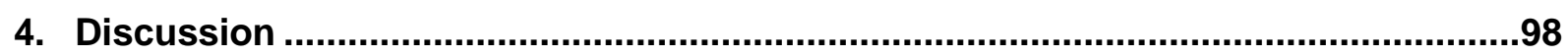

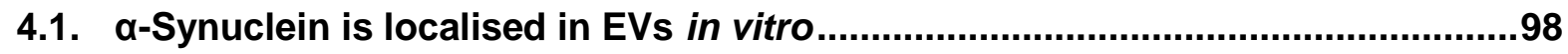

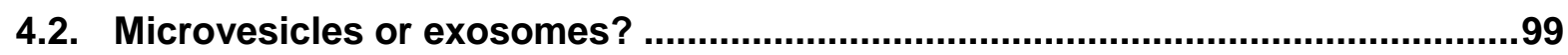

4.3. SUMO-2 interacts with phosphoinositols......................................................100

4.3.1. SUMO binding to plasma membranes is mediated by $\mathrm{PI}(3,4,5) \mathrm{P}_{3} \ldots \ldots \ldots \ldots \ldots \ldots . . . . . . .100$

4.4. $\alpha-S y n u c l e i n$ is localised in EVs in vivo...........................................................101

4.5. Extracellular vesicles as carrier for pathogenic proteins ................................101

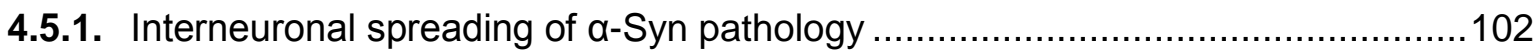

4.6. SUMO modification in neurodegenerative diseases .......................................103

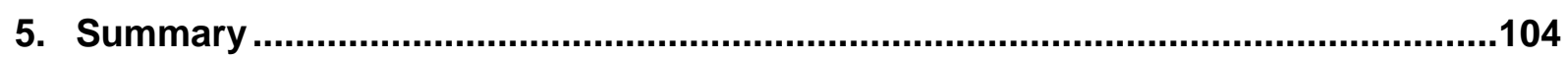

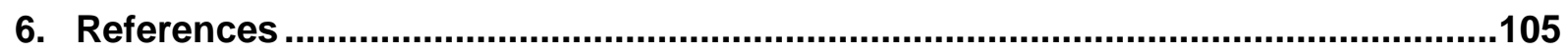

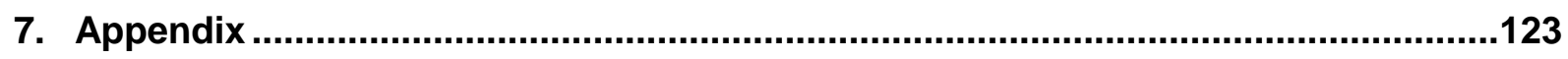

Curriculum Vitae..................................................................................................124

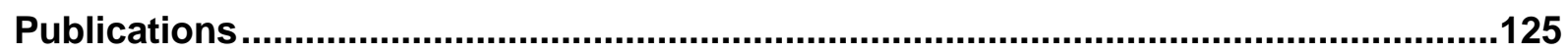




\section{List of Figures}

Fig. 1: Schematic representation of the $\alpha$-Syn amino acid sequence 3

Fig. 2: Possible mechanisms for the intercellular transmission of a-Synuclein .10

Fig. 3: Schematic view of the protein and lipid composition of exosomes. .13

Fig. 4: Biogenesis of extracellular vesicles. .14

Fig. 5: Schematic representation of the SUMO cycle 19

Fig. 6: $\alpha$-Synuclein is released in extracellular vesicles derived from N2a cells. .50

Fig. 7: Characterization of extracellular vesicles in cerebrospinal fluid .52

Fig. 8: $\alpha$-Syn is localised in the intraluminal compartment of extracellular vesicles .54

Fig. 9: Membrane binding regulates release of $\alpha$-Synuclein within extracellular vesicles .....57

Fig. 10: Membrane binding of SUMO-deficient $\alpha$-Syn mutants $2 \mathrm{KR}$ and $2 \mathrm{AA}$ .59

Fig. 11: Membrane binding of a myc-a-Syn-SUMO fusion construct .60

Fig. 12: SIFT assay for vesicle binding properties of sumoylated and non-sumoylated a-Syn.....

Fig. 13: Extracellular vesicle release of SUMOylation deficient $\alpha$-Syn

Fig. 14: Primary cortical neurons were infected with AAV to either express $\alpha$-Syn-wt or the SUMOylation-deficient mutant aSyn-2 KR .64

Fig. 15: Down-regulation of Ubc9 protein levels with siRNA. .66

Fig. 16: SUMO-2 fusion increases extracellular vesicle release of $\alpha$-Syn .67

Fig. 17: Co-expression of SUMO-2 increases release of $\alpha$-Syn with extracellular vesicles...68

Fig. 18: Co-expression of SUMO-2 does not increase the production and release of extracellular vesicles itself .69

Fig. 19: Summary of $\alpha$-Syn membrane binding and release with extracellular vesicles ........70

Fig. 20: Extracellular vesicles contain desumoylase activity ..........................................71

Fig. 21: Sumoylated $\alpha$-Syn is enriched in extracellular vesicles ......................................73

Fig. 22: SUMO-2 is released within extracellular vesicles ............................................75

Fig. 23: SUMO-2 fusion leads to extracellular vesicle sorting of GFP ..............................77

Fig. 24: Comparison of SUMO-1 and SUMO-2 release within extracellular vesicles ............78

Fig. 25: SUMO-2 increases extracellular vesicle release of the transmembrane protein APP .80

Fig. 26: Down-regulation of Tsg 101 and Alix with siRNA .82

Fig. 27: Alix and TSG101 are required for the extracellular vesicle release of SUMO-2 ......83

Fig. 28: Release of SUMO-2 with extracellular vesicles is dependent on ESCRT .84

Fig. 29: Interference with VPS4 function inhibits release of SUMO-2 within extracellular vesicles. 
Fig. 30: Mutation of the SIM motif in SUMO-2 increases extracellular vesicle release of SUMO-2 .87

Fig. 31: Microplate titration assay of SUMO-2-MFM. .89

Fig. 32: Membrane binding of SUMO-2 analysed by NMR spectroscopy .91

Fig. 33: Mutation of amino acids H16A, Q30A, F31A, K32A, I33A, H36, L42A, Y46A and D62 in the cleft and loop domains of SUMO-2 decreases membrane binding .93

Fig. 34: Mutated amino acids H16A, Q30A, F31A, K32A, I33A, H36, L42A, Y46A and D62 in the cleft and loop domains of SUMO-2 decreases extracellular vesicle sorting ...94

Fig. 35: Release of $\alpha$-Syn-SUMO-2 fusion protein with extracellular vesicles is dependent on the ESCRT .95

Fig. 36: Subcellular distribution of SUMO-2 and $\alpha$-Synuclein . .97 


\section{List of Tables}

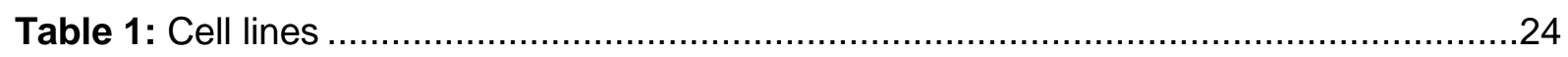

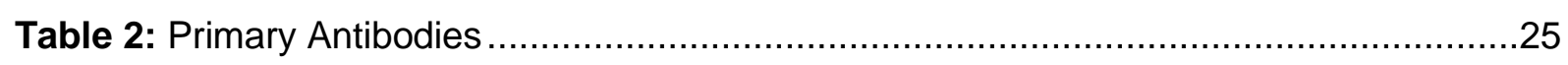

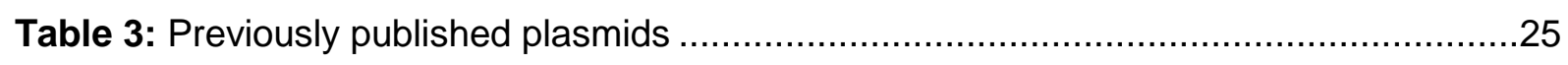

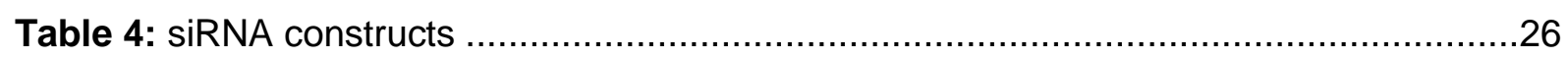

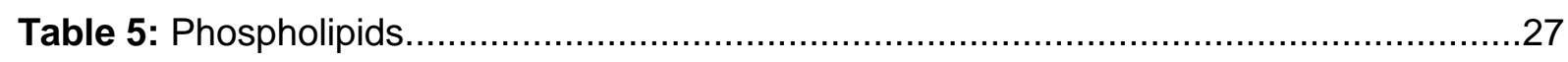

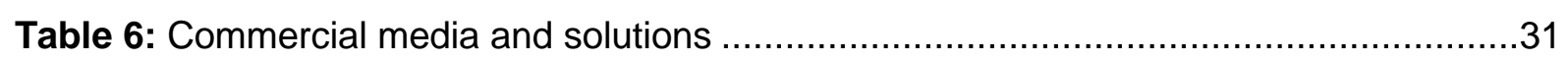

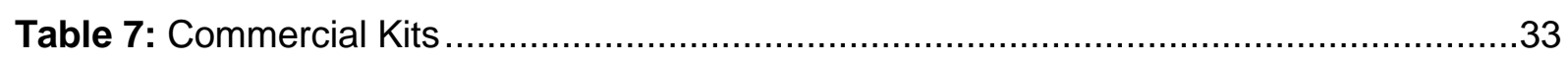

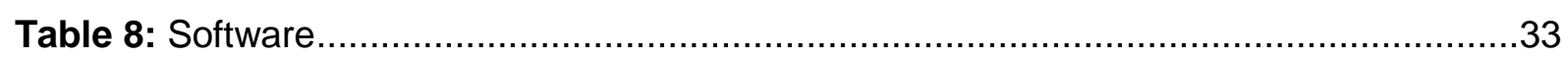

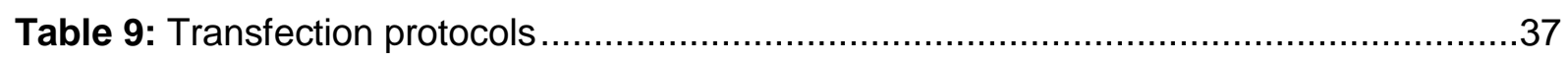

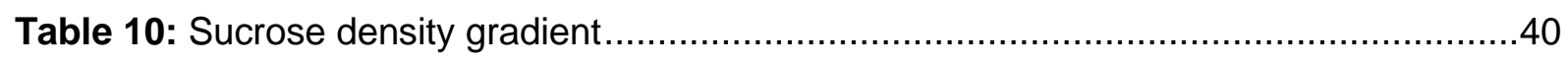

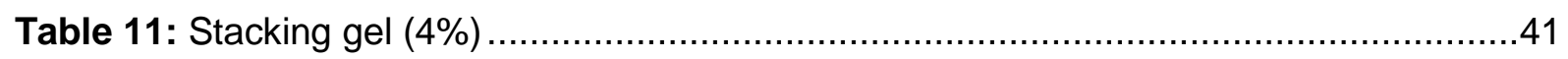

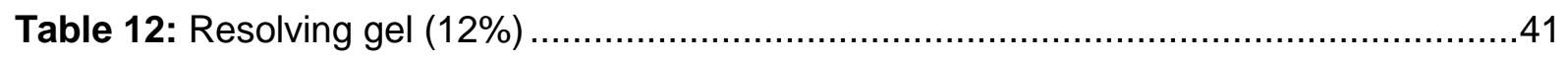

Table 13: Summary of NTA measurements ..........................................................123 


\section{IV.List of Abbreviations}

aa

$A D$

APC

APS

APP

a-Syn

AAV

$A \beta$

CHAPS

CNS

CSF

$\mathrm{CSPa}$

DABCO

DLB

DNA

DTT

E. coli

EDTA

ELISA

ER

ESCRT

ESPIT

EVs

FCS

FCS

g

GFP

$\mathrm{h}$

$\mathrm{HB}$

HBSS

HEPES

HIV

HRP

ILV

$\mathrm{kDa}$ amino acid

Alzheimer's disease

antigen presenting cell

ammonium persulfate

amyloid precursor protein

a-Synuclein

adeno-associated virus

amyloid beta protein

3-[3-(Cholamidopropyl)dimethylammonio]-1-proanesulfonate

central nervous system

cerebral spinal fluid

cysteine-string protein alpha

1,4-Diazabicyclo[2.2.2]octan

dementia with Lewy Bodies

desoxyribonuclein acid

dithiothreitol

Escherichia coli

ethylenediaminetetraacetic acid

enzyme-linked immunosorbent assay

endoplasmic Reticulum

endosomal complex required for transport

excited state intramolecular proton transfer

extracellular vesicles

fetal calf serum

fluorescence correlation spectroscopy

gramm

green fluorescent proteine

hour

homogenisation Buffer

Hanks' balanced salt solution

hydroxyethylpiperazine-N-2-ethanesulfonic acid

human immunodefciency virus

horse radish peroxidise

intraluminal vesicle

kilo Dalton 
L

LB

$\min$

MSA

MVE

NEM

NAC

NTA

PAGE

PBS

PD

PFA

PIAS

$\mathrm{PI}(3) \mathrm{P}$

$\mathrm{PI}(3,4,5) \mathrm{P} 3$

$\mathrm{PI}(3,5) \mathrm{P} 2$

$\mathrm{PI}(4,5) \mathrm{P} 2$

$\mathrm{PI}(5) \mathrm{P}$

PIPs

PLL

PLP

POPC

POPS

PTM

Rab

RanBP2

rpm

SDS

SEM

SOD1

SUMO

SIFT liter

Lewy Body

minute

multiple system atrophy

multivesicular endosome

$\mathrm{N}$-ethylmaleimide

non-amyloid beta component

nanoparticle tracking analysis

polyacrylamide gel electrophoresis

phosphate buffered saline

parkinson's disease

paraformaldehyde

protein inhibitors of activated STAT

1,2-dioleoyl-sn-glycero-3-phospho-(1'-myo-inositol-3'-

phosphate)

1,2-dioleoyl-sn-glycero-3-phospho-(1'-myo-inositol-3',4',5'-

trisphosphate)

1,2-dioleoyl-sn-glycero-3-phospho-(1'-myo-inositol-3',5'-

bisphosphate)

1,2-dioleoyl-sn-glycero-3-phospho-(1'-myo-inositol-4',5'-

bisphosphate)

1,2-dioleoyl-sn-glycero-3-phospho-(1'-myo-inositol-5'-

phosphate)

Posphatidylinositol-Phosphates

poly-L-lysine

proteolipid protein

1-palmitoyl-2-oleoyl-sn-glycero-3-phosphocholine

1-palmitoyl-2-oleoyl-sn-glycero-3-phospho-L-serine

post translational modification

Ras like protein in brain

Ran binding protein 2

revolutions per minute

Sodium dodecyl sulphate

standard error of the mean

$\mathrm{Cu} / \mathrm{Zn}$ superoxide dismutase

small ubiquitin-like modifier

scanning for intensely fluorescent targets 
SIM

SNARE

$\mathrm{SNC}$

SUV

TBS

TDP43

TEMED

Tnt

Tris

Tsg101

wt

WB

YFP
SUMO interacting motif

soluble $\mathrm{N}$-ethylmaleimide-sensitive-factor attachment receptor

substantia nigra pars compacta

Small Unilamellar Vesicle

Tris buffered saline

TAR-DNA binding protein 43

N'N'N'-tetramethylethylene diamine

tunneling nanotube

Tris(hydroxymethyl)aminomethane

tumor susceptibility gene 101

wild-type

Western blot

yellow fluorescent protein 


\section{Abstract}

Extracellular $\alpha$-Synuclein has been implicated in interneuronal propagation of disease pathology in Parkinson's disease. How a-Synuclein is released into the extracellular space is still unclear. Here, we show that $\alpha$-Synuclein is present in extracellular vesicles in the central nervous system (CNS). We find that sorting of $\alpha$-Synuclein in extracellular vesicles is regulated by sumoylation and that sumoylation acts as a sorting factor for targeting of both, cytosolic and transmembrane proteins, to extracellular vesicles. We provide evidence that the SUMO-dependent sorting utilizes the endosomal sorting complex required for transport (ESCRT) by interaction with phosphoinositols. Ubiquitination of cargo proteins is so far the only known determinant for ESCRT-dependent sorting into the extracellular vesicle pathway. Our study reveals a function of SUMO protein modification as an ubiquitin-independent ESCRT sorting signal, regulating the extracellular vesicle release of a-Synuclein. We deciphered in detail the molecular mechanism which directs $\alpha$-Synuclein into extracellular vesicles which is of highest relevance for the understanding of Parkinson's disease pathogenesis and progression at the molecular level.

We furthermore propose that SUMO-dependent sorting constitutes a mechanism with more general implications for cell biology. 


\section{Introduction}

\subsection{Synuclein}

$\alpha$-Synuclein ( $\alpha-S y n)$ is part of a protein family called the synuclein family. $\alpha$-Syn was first discovered during the purification of cholinergic vesicles from the electric organ of a Torpedo californica fish (Maroteaux et al. 1988), which was the first hint for a presynaptic role of a-Synuclein. Maroteaux and colleagues were also able to detect this protein at the nuclear envelope of neurons and therefore the researchers called the unknown protein "synuclein". Other researchers found that $\alpha$-Syn is localised in the nucleus (McLean et al. 2000, Mori et al. 2002, Goncalves et al. 2013). Later, Maroteaux et al. were able to identify another protein in rat brains which was highly homologous to a-Syn (Maroteaux et al. 1991). In amyloid plaques from Alzheimer's disease patients a peptide was identified, called non-amyloid beta component (NAC). Interestingly, the precursor protein of NAC, the NACP, was homologous to rat synuclein protein (Ueda et al. 1993). Further investigations on these synuclein proteins led to the discovery of two additional synuclein-related proteins with a length of 134 and 140 amino acids (aa) in human brain samples (Jakes et al. 1994). The protein with a length of 140 aa was found to be homolog to the precursor protein of NAC peptide (NACP). Furthermore, this human protein also shared a high conformity with the protein found in rat brains and in Torpedo californica. Thus, this protein was finally called $\alpha-S y n$. Subsequently it was possible to identify two isoforms of $\alpha$-Syn with a length of 112 aa and 126 aa which were identified as products of an alternative splicing pathway of the gene encoding for $\alpha$-Syn (Xia et al. 2001, Uversky 2007). Besides $\alpha$-Syn, the synuclein family also includes two other proteins, $\beta$-Synuclein ( $\beta$-Syn) and $\gamma$-Synuclein ( $\gamma$-Syn). $\beta$-Syn was firstly identified in extracts of human brains (Jakes et al. 1994). In $1984 \gamma$-Syn was identified as the third member of the Synuclein family with a $75.3 \%$ homology to the Torpedo californica Synuclein (Lavedan 1998, Lavedan et al. 1998).

The expression of $\alpha$-Syn and $\beta$-Syn has been primarily shown in the CNS, especially in presynaptic nerve terminals in the neocortex, hippocampus, striatum, thalamus, cerebellum, cerebellar cortex, substantia nigra and brain stem (Jakes et al. 1994, Iwai et al. 1995, Irizarry et al. 1996) and located in the cytosol. Nakajo and co-workers have shown that both proteins are located at presynaptic terminals (Nakajo et al. 1994). In contrast to $\alpha$-Syn and $\beta$-Syn, the third protein $\gamma$-Syn is mainly expressed in the peripheral nervous system (Buchman et al. 1998). Furthermore, it is known that $\gamma$-Syn is also moderately expressed in heart, skeletal muscles, and to a lesser extent in the kidney, liver and pancreas (Lavedan et al. 1998) and in many types of cancers, such as breast tumours (Jia et al. 1999). 


\subsubsection{Structure of $\alpha$-Synuclein}

a-Syn is a small 140 aa protein. It is natively unfolded and is present in neuronal cytosol and enriched in synapses. It is known that a-Syn is unstructured in aqueous solutions and that it is mainly localised to presynaptic areas, where it has also been found associated with synaptic vesicles (Cookson 2005, Lee et al. 2006). The sequence of $\alpha$-Syn can be divided into three main regions, as indicated in Fig. 1. The amino terminal region consists of 60 aa characterised by four imperfect repeats of 11 aa, with the highly conserved KTKEGV motif, which is known to bind phospholipids (Perrin et al. 2000). The binding of $\alpha$-Syn to lipids and thereby to membranes is a two-step process. The first step involves the binding of amino acids 3-25 and the second step includes a conformational shift of aa 26-100 into a helical structure. This conformational change further leads to the binding of $\alpha$-Syn to membranes in a cooperative manner (Bartels et al. 2010, Bodner et al. 2010). A lack of aa 2-19 of $\alpha$-Syn results in a decreased membrane binding of $\alpha$-Syn (Karube et al. 2008, Bartels et al. 2010), indicating that this portion of the protein mediates lipid interaction.

Point mutations

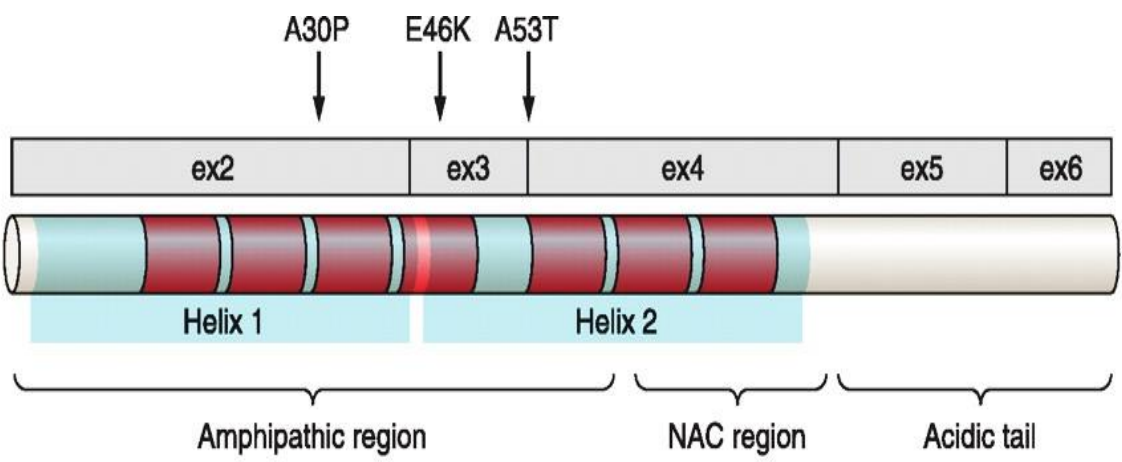

Fig. 1: Schematic representation of the $\alpha$-Syn amino acid sequence

a-Syn consist three main domains. The $\mathrm{N}$-terminally located amphipathic region, the hydrophobic NAC domain in the centre and an acidic C-terminal domain. Autosomal dominant mutations found in familial cases of PD are indicated with arrows. Adapted and modified from (Corti et al. 2011).

A hydrophobic centre domain corresponding to the NAC domain is located between aa 61-95. The NAC region contains two additional imperfect repeats and is believed to form $\beta$-rich fibrils of $\alpha$-Syn. The aa 71-82 within the NAC are mainly responsible for fibril formation of $\alpha$-Syn (Bodles et al. 2001, Giasson et al. 2001, Uversky et al. 2002) and deletion of aa 71-82 in human $\alpha$-Syn prevents the protein's aggregation (Giasson et al. 2001). Furthermore, the NAC domain shares sequence homology with the aa sequence which is responsible for the aggregation of other amyloidogenic peptides, such as $A \beta$ and prion protein (El-Agnaf et al. 1998). Aa 96-140 represents a highly negatively charged region at the C-terminal end of a-Syn which mainly consists of acidic residues. 
It has been shown that a C-terminally truncated version of $\alpha$-Syn is able to aggregate more rapidly compared to the full length protein, indicating that the $\mathrm{C}$-terminus might play a role in the regulation of $\alpha$-Syn aggregation (Murray et al. 2003).

\subsubsection{Posttranslational modifications of $\alpha$-Syn}

The C-terminus of $\alpha$-Syn is a potential target for post-translational modifications (PTM) of a-Syn. It has been shown that phosphorylation is the most common posttranslational modification of $\alpha$-Syn, which predominantly occurs at aa S129 and to a lesser extent at S87 and additionally at the aa residues Y125, Y133 and Y135 (Okochi et al. 2000, Nakamura et al. 2001). Furthermore, insoluble $\alpha$-Syn is extensively phosphorylated at Ser 129 in DLB brain tissue (Fujiwara et al. 2002). It has been shown that under physiological conditions only $4 \%$ of the soluble monomeric form of $\alpha$-Syn appears phosphorylated in vivo. In contrast to this, $90 \%$ of $\alpha$-Syn in its aggregated form is phosphorylated in Lewy bodies (LB) (Fujiwara et al. 2002, Anderson et al. 2006). This fact lead to the assumption that the phosphorylation of a-Syn at aa S129 directly correlates with the aggregation level of a-Syn (Tenreiro et al. 2014).

Another post-translational modification of $\alpha$-Syn is the nitration of aa residues $Y 39, Y 125$, $\mathrm{Y} 133$ and $\mathrm{Y} 136$, these residues are also predominantly located in the C-terminal region of a-Syn (Giasson et al. 2000). Interestingly, high concentrations of nitrated a-synuclein have been found in LB (Giasson et al. 2000). During increased oxidative stress conditions it has been shown that $\alpha$-Syn is nitrated to a higher level, suggesting an important role of oxidative stress in LB diseases (Kim et al. 2014). Furthermore, in vitro studies on the nitration of $\alpha$-Syn have shown that nitration is able to induce the oligomerisation of $\alpha$-Syn, which is leading to mitochondrial defects and results in apoptosis and cell death (Liu et al. 2011), as well as the overproduction of nitric oxide, which mediates the increase of neurotoxic a-Syn species (Danielson et al. 2009). Another PTM of $\alpha$-Syn is SUMOylation. Dorval and Co-workers postulate that $\alpha-S y n$ is preferentially sumoylated by SUMO-1 (Dorval et al. 2006).

In contrast to this, Krumova and colleagues have shown that $\alpha$-Syn can be modified by SUMO-1 and SUMO-2 in different cell lines (Krumova et al. 2011). In addition they demonstrate that $\alpha$-Syn is sumoylated in rat brains in vivo and additionally that covalent attached SUMO is able to regulate aggregation induced toxicity of $\alpha$-Syn (Krumova et al. 2011). 


\subsubsection{Function of $\alpha$-Synuclein}

a-Syn plays a major role in a variety of neurodegenerative diseases, so called Synucleinopathies. The exact physiological role of $\alpha$-Syn remains poorly understood. As indicated above, $\alpha$-Syn is able to bind to membranes (lipids); together with the findings of Maroteaux et al. in 1988 that showed synaptic localisation of Synuclein protein, this led to the assumption of a synaptic function of $\alpha$-Syn. Interestingly, it has been shown by Abeliovich et al. that $\alpha$-Syn deficient mice display a reduction in the levels of striatal dopamine including released dopamine (Abeliovich et al. 2000).

Overexpression of $\alpha$-Syn in yeast resulted in the appearance of cytosolic lipid inclusions and the accumulation of vesicles, indicating impaired ER-Golgi trafficking induced by a-Syn (Outeiro et al. 2003). This notion was supported by the finding that overexpression of the small GTPase Rab1 could partially restore $\alpha$-Syn toxicity and the $\alpha$-Syn induced block of ERGolgi trafficking (Outeiro et al. 2003, Cooper et al. 2006, Gitler et al. 2008).

Similar results have been also reported in non-neuronal cell lines (Thayanidhi et al. 2010), in the nematode Caenorhabditis elegans (Cooper et al. 2006, Gitler et al. 2008, Kuwahara et al. 2008, van Ham et al. 2008) and as well in Drosophila melanogaster (Cooper et al. 2006). These data lead to the assumption that $\alpha$-Syn might play a role in the blocking of vesicle trafficking pathways. The majority of $\alpha$-Syn is physiologically located at distal pre-synapses. Scott and Co-workers suggested that impairment of vesicle trafficking first occurs at synapses and might be mediated by neuronal $\alpha$-Syn (Scott et al. 2012). They found that an excess of $\alpha$-Syn is involved in the impaired mobility of recycling pool vesicles and also inhibits inter-synaptic trafficking (Scott et al. 2012). Furthermore, an additional study has shown that the over-expression of $\alpha$-Syn significantly inhibits the release of neurotransmitters, mediated through a significant reduction in the amount of the vesicle recycling pool (Nemani et al. 2010). Additionally, Nemani and co-workers ruled out by ultrastructural analysis that an over-expression of $\alpha$-Syn also resulted in a reduction of the density of synaptic vesicles in the active zone, combined with an impairment of vesicle reclusteringafter endocytosis (Nemani et al. 2010) These findings are consistent with the previous findings of Scott et al. In conclusion it can be assumed that $\alpha$-Syn plays a role in synaptic vesicle trafficking although a-Syn knockout mice display no obvious phenotype (Abeliovich et al. 2000). However, subtle memory deficits were recently described in these animals, supporting a potential function of a-Syn in synapse function (Kokhan et al. 2012). Further research will be needed to uncover to complete physiological role of $\alpha$-Syn. 


\subsection{Synucleinopathies}

Neurodegenerative diseases which are characterised by the pathological aggregation of $\alpha$-Syn are termed synucleinopathies. Filamentous intracytoplasmic $\alpha$-Syn inclusions are called Lewy bodies and Lewy neuritis, which are the pathological hallmarks in Parkinson's disease (PD) and dementia with Lewy bodies (DLB) (Spillantini et al. 1997, Spillantini et al. 1998b). Multiple system atrophy (MSA), a disease which is characterised by oligodendroglial inclusions of a-Syn, so called Papp-Lantos bodies (Spillantini et al. 1998a, Tu et al. 1998, Wakabayashi et al. 1998), also belongs to the group of synucleinopathies.

\subsubsection{Parkinson's disease}

Parkinson's disease (PD) is one of the most common neurodegenerative disorders affecting $1-2 \%$ of the global population at the age of 65 years (de Lau et al. 2006) and about $5 \%$ of the individuals older than 85 years of age. PD is a progressive disease characterised by a specific loss of neurons, most notably dopaminergic neurons in the substania nigra pars compacta (SNc) of basal ganglia in the midbrain. The primary symptoms in PD, which occur due to the neuronal loss, are severe motordeficits including bradykinesia, postural instability, rigidity and resting tremor, usually accompanied with a shuffling gait. The first evidence of an involvement of a-Syn pathology in PD came up in 1997,due to the identification of the missense mutation A53T in the a-Syn gene locus (SNCA) of familial PD patients (Polymeropoulos et al. 1997). Moreover, Spillantini and colleagues could demonstrate that a-Syn is the major component of Lewy bodies (Spillantini et al. 1997). In further investigations two additional mutations in the SNCA were discovered. In 1998 Krüger et al. discovered the familial A30P mutation and six years later the E46K mutation in SNCA was indentified (Zarranz et al. 2004). In addition it has been shown that triplication of the $\alpha$-Syn wt form is also responsible for autosomal dominant forms of PD (Krüger et al. 1998, Zarranz et al. 2004).

\subsubsection{Familial Parkinson's disease}

Genetically induced cases of PD are relatively rare, compared to sporadic PD cases. Several gene mutations have been described in patients with a familial form of PD. Three of the most prominent mutations are already mentioned in section 1.2.1 (A53T, A30P and E46K). In addition to mutations of the SNCA genes, mutations in the Parkin (PARK2) gene have been identified, as a potential source for an early onset Parkinsonism (Klein et al. 2007). 
Interestingly, a mutation in the PARK2 gene causes similar symptoms compared to idiopathic PD patients. Another mutation which causes familial PD has been identified in a German family in the UCH-L1 (PARK5) gene, which is encoding for the ubiquitin C-terminal hydrolase-1 (Leroy et al. 1998). Additional mutations have been found in PINK1 (PARK6) (Valente et al. 2002a, Valente et al. 2002b), the DJ-1 gene (PARK7) (Bonifati et al. 2003) and the LRRK2 gene (PARK8) (Mata et al. 2006).

\subsubsection{Dementia with Lewy bodies (DLB)}

DLB was described as a neurodegenerative dementia with Lewy body pathology (McKeith et al. 2005). Clinically, DLB is characterized by early cognitive impairment, visual hallucinations, Parkinson syndrome, REM sleep behavior disorder and fluctuating cognition and alertness and neuroleptic sensitivity (McKeith et al. 2006). The distribution of Lewy body pathology differs from that observed in PD and includes cortex and brainstem (McKeith et al. 2005). Some patients with PD will progress towards PD dementia (PDD) which is paralleled by an emerging cortical distribution of Lewy body pathology. This led to the assumption that PDD and DLB may represent a disease continuum rather than 2 distinct disease entities (Donaghy et al. 2014).

\subsubsection{Multiple system atrophy (MSA)}

MSA is a progressive neurodegenerative disorder characterised by a Parkinson syndrome,, cerebellar symptoms, autonomic failure. Neuronal loss was observed in the substantia nigra, the cerebellum, the pons and in the spinal cord (Bendor et al. 2013). In contrast to DLB and PD, a-Syn deposits predominantly occur in oligodendroglia rather than in neurons (Kim et al. 2014). This is followed by demyelination and subsequent neurodegeneration (Baker et al. 2006, Song et al. 2007, Huang et al. 2008). In contrast to PD, no familial mutations are known in the case of MSA (Ozawa et al. 1999, Morris et al. 2000, Jin et al. 2008). 


\subsection{Spreading of disease pathology}

In many neurodegenerative disorders, misfolded proteins play an important role in the pathogenesis. The misfolding of these proteins promotes the fibrillar aggregation of these proteins which are neuropathological hallmarks of the respective diseases.

\subsubsection{Permissive templating}

The concept of permissive templating of protein misfolding and aggregation in neurodegenerative diseases is widely discussed. Permissive templating describes the induction of a disease-causing confirmation by exposure of a protein to a misfolded seed, occurring in a susceptible environment. This is followed by abnormal aggregation. Induced aggregates can initiate misfolding of further proteins. Therefore, the process can proceed independently of the initial pathogenic protein, because the pathogenesis, if once initiated, becomes self-propagating (Hardy 2005). The process is characterised by a propagation phase in which the native protein will be changed to pathogenic seed, which is mediated by efficient templating of the native protein (Hardy 2005).

In the case of $\alpha$-Syn it has been assumed that misfolded, monomeric $\alpha$-Syn can act as a template for other monomeric $\alpha$-Syn species, to convert the non-pathogenic $\alpha$-helix form into the pathogenic $\beta$-sheet rich structure of $\alpha$-Syn (Brundin et al. 2008). This is consistent with the assumption that $\alpha$-Syn fibrils or rather their breakdown products are able to act as seeds. These seeds can further interact with monomeric a-Syn and are capable to induce the fibrillization of monomeric $\alpha$-Syn species (Wood et al. 1999). According to these findings, it has been shown that seeds derived from the A30P mutant version of $\alpha$-Syn are able to convert wt a-Syn into A30P fibrils (Yonetani et al. 2009). The process described above is comparable to the templated conversion of the non-infectious prion protein $\operatorname{PrP}^{\mathrm{c}}$ to the infectious scrapie form $\operatorname{PrP}^{\mathrm{Sc}}$ (Angot et al. 2010). 


\subsubsection{Transmission of $\alpha$-Synuclein in a prion-like manner}

Braak and Co-workers have shown that the progression of $\alpha$.Syn pathology seems to follow a stereotypical anatomical path throughout the brain. According to Braak et al, $\alpha$-Syn pathology starts in the nucleus vagus from where it spreads to the substantia nigra, followed by spreading to higher basal ganglia and neocortical regions of the brain (Braak et al. 2003). The notion of intracerebral propagation of $\alpha$-Syn pathology gained much attention following in vivo evidence of interneuronal diseases propagation in human brains. In these studies the researchers transplanted successfully foetal dopaminergic neurons in patients with PD pathology, to compensate for the loss of dopaminergic neurons in the substantia nigra (Bjorklund et al. 2003, Olanow et al. 2003, Kordower et al. 2008a, Kordower et al. 2008b, Li et al. 2008, Mendez et al. 2008). The grafted neurons showed a robust survival and no loss in dopaminergic activity when the tissue was analysed 18 months after the surgery when one of the patients died (Kordower et al. 1995). In contrast, in post mortem brain tissues of several patients who died 14 years after the transplantation, the grafted neurons revealed Lewy body pathology (Kordower et al. 2008a, Kordower et al. 2008b, Li et al. 2008), as assessed by $\alpha$-Syn, $\alpha$-Syn S129p and Thioflavin staining. The obtained data by Kordower et al. and Li et al. supporting the idea of cell to cell transfer of a-Syn in vitro and in vivo, lead to the assumption of an intercellular (interneuronal) spreading of PD disease pathology Recently, several studies with cell culture and animal models have found evidence for transcellular spreading and induction of aggregation of $\alpha$-Syn (Hansen et al. 2011, Rey et al. 2013, Ulusoy et al. 2013, Luk et al. 2014, Recasens et al. 2014).

Fig. 2 displays a short overview of possible mechanisms for the intercellular (interneuronal) transmission of a-Syn including tunnelling nanotubes (Tnt), active and passive secretion of aSyn or extracellular vesicles (EVs). Tnts are thin extensions of cell membranes that are able to connect cells over long distances. It is known that these tubes can develop by subsequent membrane fusion, during cell division, or via Actin mediated overlapping from one cell to another cell (Angot et al. 2010). However, in contrast to Huntington (Costanzo et al. 2013), $\alpha$-Syn has never been observed in Tnts. Another possibility is the uptake of free interstitial $\alpha-$ Syn, which is released after either active secretion or by passive release from a dying neuron followed by uptake through a healthy neuron. Recently, Ulusoy et al. have shown that interneuronal spreading of $\alpha$-Syn is an active process which requires living neurons (Ulusoy et al. 2015). This makes a passive release from dying neurons a less likely mechanism. Alternatively, $\alpha$-Syn could be released within EVs from one cell and could be taken up by another. This could explain the directional spreading of disease pathology because EVs can carry targeting signals for cellular delivery. In addition, they could efficiently transfer large amounts of a-Syn. 


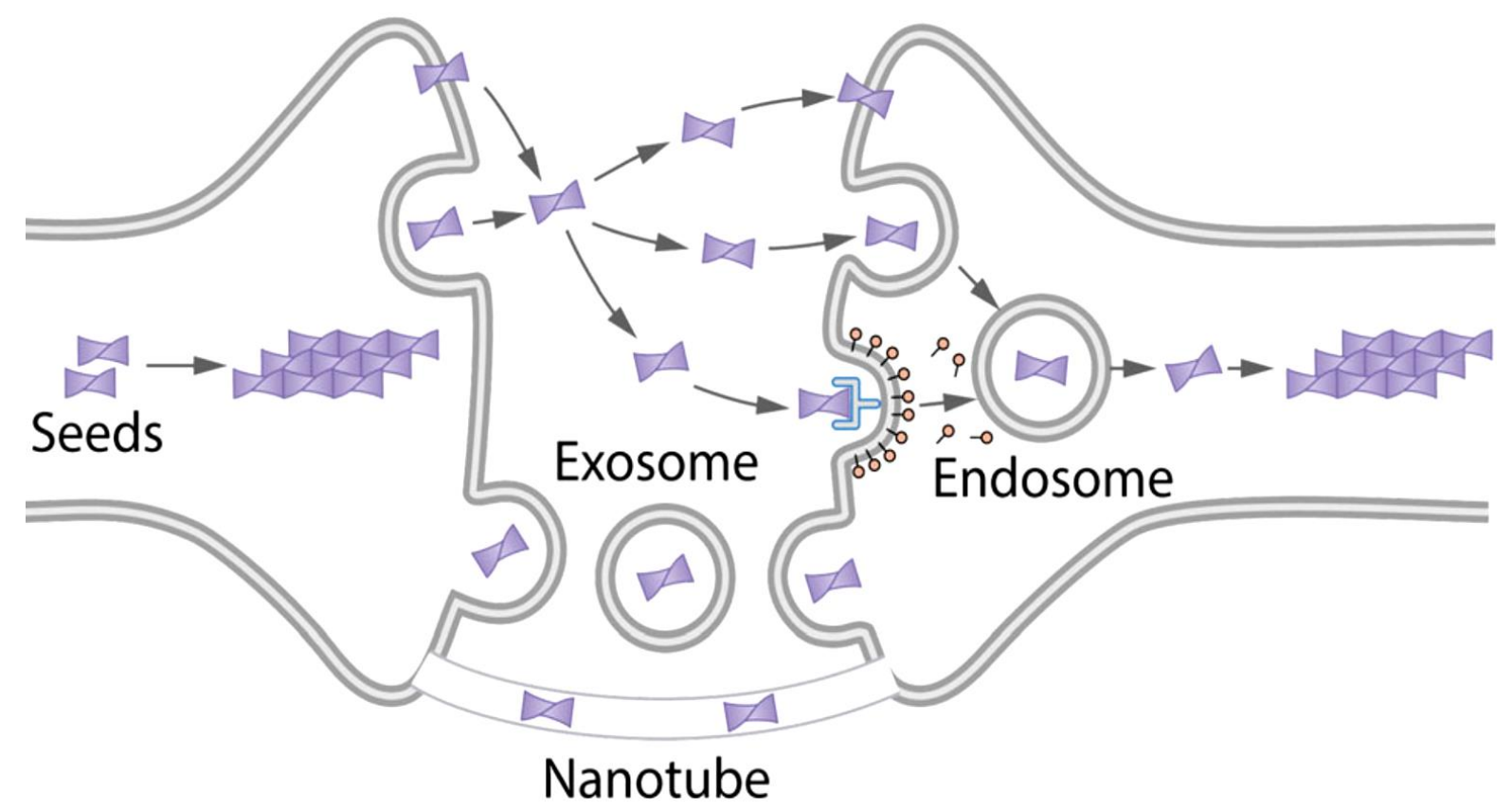

Fig. 2: Possible mechanisms for the intercellular transmission of $\alpha$-Synuclein

Potential seeds of $\alpha$-Syn can be released to the extracellular space within exosomes (extracellular vesicles) or either in a free form. Seeds which are released within vesicles can later fuse with the plasma membrane of another neuron and release the seeds, which can further act as seeds for additional $\alpha$-Syn molecules. Seeds which are not bound to membranes of vesicles might be able to directly penetrate plasma membranes of a recipient cell. Proteins could be additional transferred by tunnelling nanotube from one neuron to another. Adapted and modified from (Goedert et al. 2014). 


\subsection{Extracellular vesicles}

The communication between cells is essential for all eukaryotic organisms. Eukaryotic cells are divided in various cellular compartments, which consist of membrane enclosed organelles. Cells are in constant contact with their environment and with other cells. This exchange of information can be mediated via direct contact or via the transfer of secreted signal molecules, like cytokines, chemokines and the uptake of secreted molecules from other cells. (Keller et al. 2006, Raposo et al. 2013). In the last decades another mechanism has been intensively discussed, the intracellular communication via transfer of extracellular vesicles from one cell to another. A variety of different etxracellular vesicles are known today. Extracellular vesicles include microvesicles, exosomes and apoptotic bodies (Holme et al. 1994, Hess et al. 1999, Cocucci et al. 2009, György et al. 2011). Microvesicles or shedding vesicles have a diameter of $40-1000 \mathrm{~nm}$ and are derived by shedding directly from the plasma membrane. In contrast, exosomes are vesicles with a diameter of $40 \mathrm{~nm}-100 \mathrm{~nm}$ which are formed by inward vagination of the limiting membrane of late endosomes, giving rise to intraluminal vesicles (ILVs). The ILV filled endosomes are then termed multivesicular endosomes. Upon their fusion with the plasma membrane, ILVs are released to the extracellular space as exosomes. They were first described to be released during reticulocyte differentiation (Harding et al. 1983, Pan et al. 1983, Harding et al. 1984, Pan et al. 1985). (Johnstone et al. 1987). Based on morphology or biochemical properties it is not possible to distinguish between exosomes and microvesicles (Raposo et al. 2013). Therefore, there is now a consensus to term these vesicles extracellular vesicles (EV). EVs are released by a variety of different cells and are present in different body fluids including semen (Park et al. 2011, Aalberts et al. 2012), blood (Caby et al. 2005), urine (Pisitkun et al. 2004) and CSF (cerebrospinal fluid) (Vella et al. 2008).

\subsubsection{Morphology and composition of extracellular vesicles}

\section{Morphology}

Extracellular vesicles can be analysed via electron microscopy, where they occur in a typical cup-shaped morphology with a lipid bilayer in a diameter of $50-100 \mathrm{~nm}$, which is consistent with the observed morphology of intraluminal vesicles inside (ILVs) of MVBs (multivesicular bodies) (Fauré et al. 2006). Extracellular vesicles can be purified from conditioned cell culture medium and a variety of biological fluids via ultracentrifugation approach at $100.000 \times g$ (Théry et al. 2006). 
With this ultracentrifugation protocol it is possible that other small vesicles with a similar size might simultaneously be collected. To validate the purity of the extracellular vesicle preparation subsequent methods may be used. Thus, a sucrose density gradient is often used to obtain a relatively purer preparation of EVs. In a sucrose density ultracentrifugation approach organelles derived from the Golgi apparatus, or the ER, protein aggregates and several other contaminations, show different and specific floating behaviors, which allows for the accurate separation of potential contaminations from the exosomal fraction. It is known that in sucrose gradients EVs in the size range of $40-100 \mathrm{~nm}$ are floating at densities of 1.13 - $1.19 \mathrm{~g} / \mathrm{mL}$ (Raposo et al. 1996, Zitvogel et al. 1998, Théry et al. 2006). In contrast, contaminations derived from the ER are found to float at densities of $1.18-1.25 \mathrm{~g} / \mathrm{mL}$ (Théry et al. 2006), vesicles from the Golgi apparatus are known to float at densities around $1.05-1.12 \mathrm{~g} / \mathrm{mL}$ (Théry et al. 2006) and big apoptotic bodies float at higher densities around $1.3-2 \mathrm{~g} / \mathrm{mL}$, depending on their size (Gutwein et al. 2005).

\section{Composition of extracellular vesicles}

The protein and lipid composition of EVs depends on the releasing cell. Fig. 3 provides an overview of proteins, nucleic acids and lipids which have been identified within EVs. Proteins which are responsible for MVB formation and involved in the ESRCT complex (endosomal complex required for transport) are highly abundant in EVs (e.g. Alix and Tsg101) (van Niel et al. 2006). Another important group of proteins which are also associated with EVs in lipid rafts are so called Flotillins (Parolini et al. 2009).

Extracellular vesicles also contain heat shock proteins (e.g. like Hsp70 and Hsp90), which are known to permit peptide loading on major histocompatibility complex MHC-I and MHC-II (Gastpar et al. 2005). Notably, histocompatibility complexes are found to be highly enriched in exosomes that are released by parental cells from the immune system (Thery et al. 2001a, Thery et al. 2001b). Furthermore EVs are enriched in Integrins and Tetraspanins, like CD9, CD81, CD82 and CD63 (Schorey et al. 2008). 


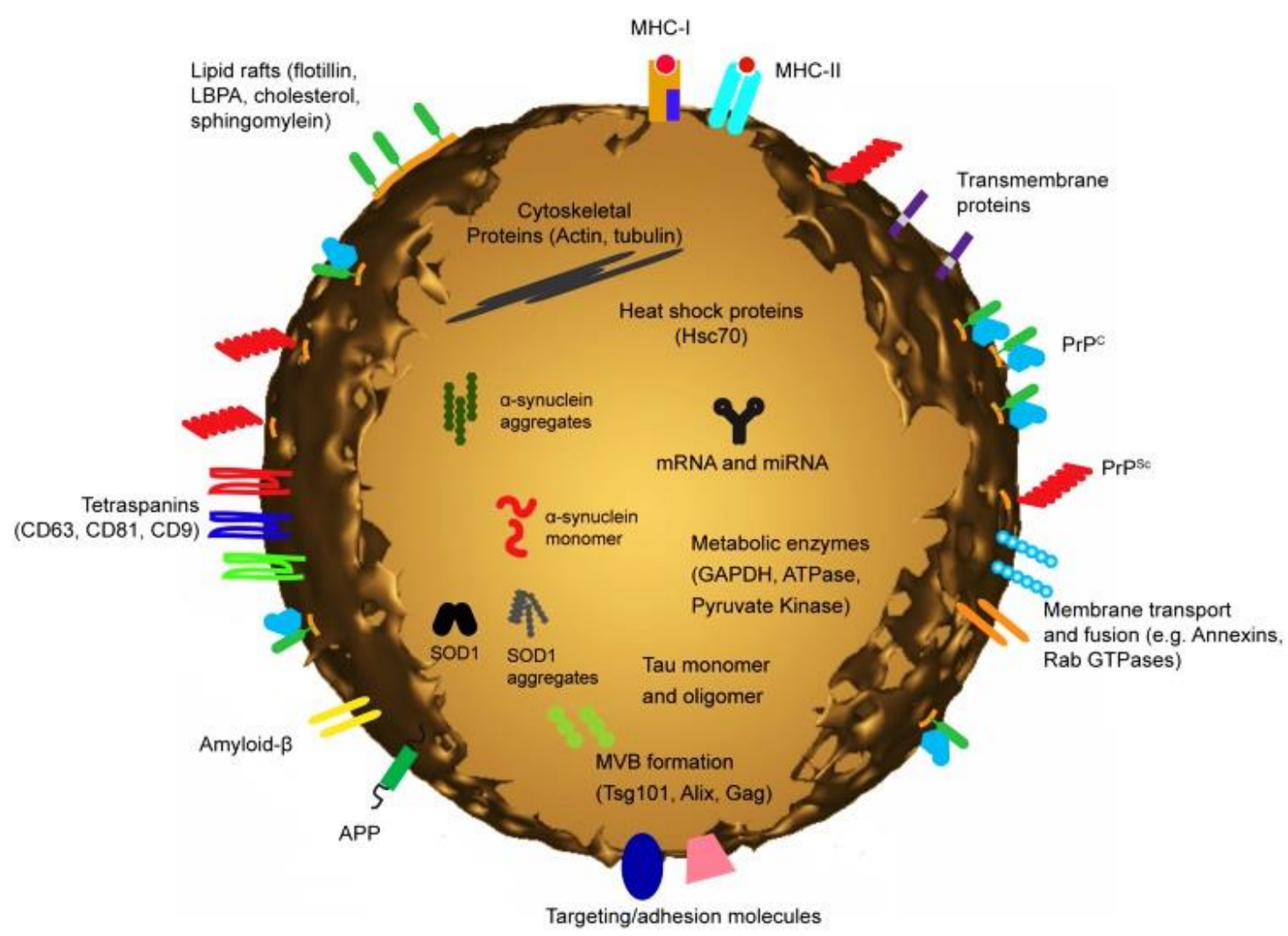

Fig. 3: Schematic view of the protein and lipid composition of exosomes

Common overview of proteins and lipids which can be resides within exosomes or attached to the outer membrane. Exosomes contain a huge quantity of proteins depending in their releasing parental cell types. Additionally they also contain nucleic acids, like different RNA species. In addition to general proteins which are involved of the formation of MVBs, exosomes can also contain proteins that are associated with neurodegenerative diseases, like PD, AD and prion diseases as well. Adapted from (Bellingham et al. 2012).

Different studies revealed that EVs are also highly enriched with different lipid molecules. EVs contain high amounts of cholesterol, sphingomyelin, ceramides, diglyceride, phosphatidylcholine, phosphatidylethanolamine and phosphatidylserine (Wubbolts et al. 2003, Laulagnier et al. 2004, Subra et al. 2007, Brouwers et al. 2013) as well as lipid-rich microdomains (de Gassart et al. 2003). The EV marker protein Flotillin-2 is known to be associated with cholesterol-rich membrane microdomains. Several studies demonstrated that EVs can also carry different RNA species, like messenger RNAs (mRNAs) and micro RNA (miRNAs) (Ratajczak et al. 2006, Valadi et al. 2007, Hunter et al. 2008, Rabinowits et al. 2009, Michael et al. 2010). 


\subsubsection{Biogenesis of extracellular vesicles}

\section{Exosomes}

Exosomes are generated in cells within the endosomal system which is composed of primary endocytic vesicles, early and late endosomes and lysosomes (Mellman 1996). During endosome maturation an accumulation of vesicles occurs inside the late endosomes. These vesicles are formed by inward budding of the limiting membrane and are termed intraluminal vesicles (ILV). Late endosomes filed with ILVs are also called multivesicular bodies (MVBs) (Fevrier et al. 2004a). The MVBs can later fuse with the plasma membrane and ILVs can be released to the extracellular space as exosomes (Fig. 4). An alternative pathway is the fusion of MVBs with lysosomes for subsequent degradation of ILVs (Luzio et al. 2010). Based on morphology and biophysical properties, exosomes cannot be distinguished from shedding vesicles/microvesicles which bud from the plasma membrane. Therefore, we will use the term extracellular vesicles (EVs) throughout the text.

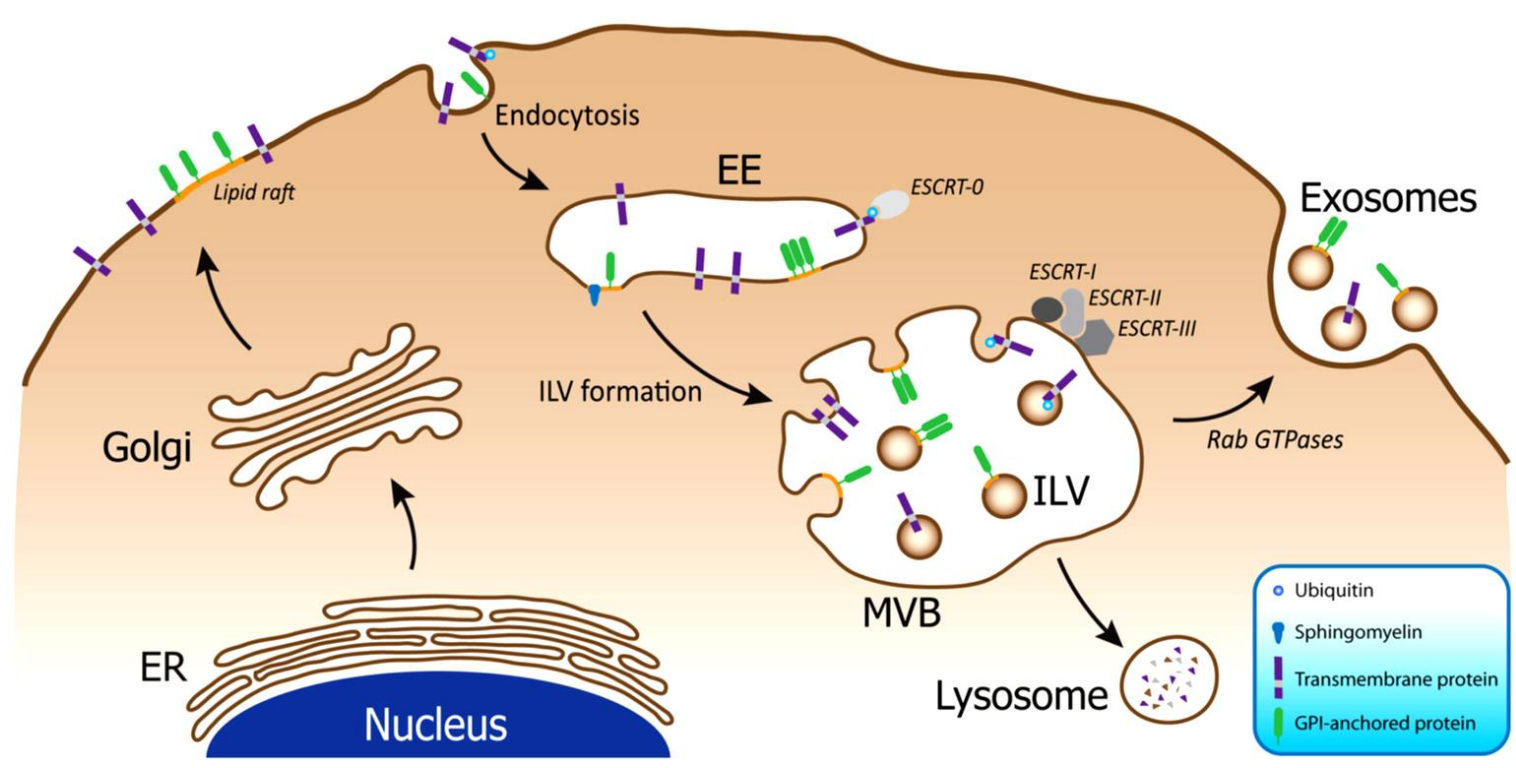

Fig. 4: Biogenesis of extracellular vesicles

Exosomes are formed within the endosomal system by inward invagination of the limiting membrane of late endosomes. This gives rise to intraluminal vesicles. The late endosome which is filled with intraluminal vesicles is then called multivesicular body. After fusion of the multivesicular body with the plasma membrane, these vesicles are released to the extracellular space as exosomes. Additionally MVBs can also fuse with lysosomes for the degradation of their cargo content. The sorting of proteins into exosomes and the biogenesis of exosomes requires the ESCRT-complexes 0 to III. Later the attachment of the MVBs and the resulting release of exosomes require several Rab GTPases. Adapted from (Bellingham et al. 2012) 


\section{ESCRT dependent sorting of proteins}

So far, no consensus sequence for protein sorting into EVs has been identified. Posttranslational modification by monoubiquitination has been shown to direct the sorting of these proteins to the ESCRT machinery (Babst 2011, Piper et al. 2011). The ESCRT consists of subcomplexes O, I, II and III. According to Hurley and Co-workers (2008), the ESCRT complexes 0 -II are responsible for the recognition and sorting of ubiquitinated proteins to ILVs. Subsequent budding of vesicles from the plasma membrane is driven by the ESCRT-III complex (Hurley 2008, Hurley 2010).

The protein hepatocyte growth factor-regulated tyrosine kinase substrate (Hrs) is able to bind mono-ubiquitinated proteins and subsequently forms a complex with the proteins Signaltransducing adaptor molecule (STAM), clathrin (Raiborg et al. 2003) and Esp15. HRS binds via its FYVE domain to the endosome specific lipid phosphatidylinositol-3-phosphate (PI(3)P) and is therefore localized to early and late endosomes (Misra et al. 1999). Later the ESCRT-I is recruited and the protein Tsg101 is supposed to form a complex with ubiquitinated cargo proteins, which subsequently binds the protein Alix/AIP, leading to the recruitment of ESCRT-II. The binding of ESCRT-II initiates the oligomerisation of small coiled proteins and finally the recruitment of ESCRT- III. This complex is then responsible for the binding of the de-ubiquitinating enzyme Doa4, which removes the ubiquitin tag from the cargo proteins and finally initiates membrane budding to form ILVs. At the end of this sorting process the activity of the AAA-ATPase Vps4 is responsible for the final fission and disassembly of the ESCRTcomplexes (Babst et al. 1998, Raiborg et al. 2003, Yeo et al. 2003, Fevrier et al. 2004a, Babst 2005, Keller et al. 2006). Recent observations revealed that ubiquitination of cargo proteins may be not the only determining factor for an interaction with the ESCRT machinery. For instance, the ESCRT-dependent sorting of the T-cell co-receptor CD4 or the delta opioid receptor DOR are not dependent on ubiquitination (Shields et al. 2011). It is, however, unclear, whether this reflects an ubiquitin independent ESCRT interaction. It is possible that both proteins bind to ubiquitinated interaction partners which mediate ESCRT dependent sorting. Thus, it is still not known whether ubiquitin-independent sorting mechanisms to the ESCRT-pathway exist. Recently, Villarroya-Beltri and co-workers demonstrated that the sumoylated heterogeneous nuclear ribonucleoprotein A2B1 (hnRNPA2B1), is able to interact with specific miRNA motifs. This interaction regulated the loading of these miRNAs into exosomes. Interestingly, it was shown that hnRNPA2B1 is sumoylated in EVs (VillarroyaBeltri et al. 2013). Based on these finding it is possible that the small ubiquitin like modifier (SUMO) could act is an ubiquitin-independent sorting determinant for the ESCRT-pathway. 


\section{ESCRT-independent sorting of proteins}

ESCRT-independent sorting into extracellular vesicles was first shown for the Melanosomal protein Pmel17 (de Gassart et al. 2003, Theos et al. 2006) via a luminal domain dependent pathway (Theos et al. 2006). Contrary to these findings, it has been shown that the tetraspanin CD63 is involved in the endosomal sorting of PMEL during melanogenesis, in a ESCRT-dependent and independent manner as well (van Niel et al. 2011). Other mechanisms of ESCRT-independent sorting include interaction with tetraspanins and a ceramide-dependent pathway. Trajkovic and co-workers showed in 2008 that the ESCRT proteins Alix and Tsg101 were not involved in the sorting of the proteolipid protein (PLP). In contrast they observed that the EV release of PLP is mediated by ceramide-induced inward budding of intraluminal vesicles. Ceramide is known to have a cone-shaped morphology, which may favour the membrane invagination of late endosomal membranes to form ILVs (Trajkovic et al. 2008). Other studies found higher order oligomerisation to play a role the in sorting of proteins for EV release (Fang et al. 2007).

\subsubsection{Secretion of extracellular vesicles}

It has been shown that different Rab proteins are able to regulate the EV release from different types of cells. (Ostrowski et al. 2010). The secretion of EVs into the extracellular space is finally driven by the fusion of MVBs with the plasma membrane. It is known that this process possibly involves different SNARE proteins (Soluble $\mathrm{N}$-ethylmaleimide-sinsitive factor attachtment protein receptors) (Pelham 2001). According to the literature, vesicular SNAREs ( $v$-SNAREs), are localised to MVBs and are able to interact with target SNAREs (tSNAREs). Both can form a membrane bridging complex and this complex can mediate the membrane fusion (Chaineau et al. 2009). As reported by Fader and co-workers in 2009, the V-SNARE complex was responsible for the fusion of MVBs with the plasma membrane in an erythroleukemia cell line ((TI-VAMP/VAMP7) vesicle associated membrane protein) (Fader et al. 2009). 


\subsubsection{Function of extracellular vesicles}

Originally, it was assumed that EVs serve to discard obsolete proteins such as cytoplasma and plasma membrane during reticulocyte maturation (Johnstone et al. 1987). It has now become increasingly clear that EVs are involved in a variety of physiological processes, including intercellular communication (Colombo et al. 2014). Different studies indicate that tetraspanins alone or together with Integrins can mediate specific target cell delivery of EVs (Rana et al. 2011, Rana et al. 2012). EVs can either be internalized by endocytic uptake or direct fusion with the plasma membrane (Raposo et al. 2013). In addition to protein transfer, EVs are also able to deliver nucleic acids, thereby leading to changes in protein expression. E.g., Valadi and Co-workers described the transfer of mRNA from murine to human mast cells via exosomes and the subsequent translation of mouse protein in the recipient human mast cells (Valadi et al. 2007). In vivo evidence of a functionally active transfer of small RNAs and miRNAs mediated by EVs was described (Pegtel et al. 2010, Zomer et al. 2010). EVs are released by immune cells and can modulate inflammatory response (Braicu et al. 2015). For example, EVs are released by antigen presenting cells (APCs), like B-lymphocytes and dendritic cells which carry factors for T-Cell stimulation and MHCs, finally leading to T-cell activation (Raposo et al. 1996). (Wolfers et al. 2001, Giri et al. 2008, Théry et al. 2009, Walker et al. 2009). Other functions of EVs include morphogenesis (Sheldon et al. 2010, Gross et al. 2012, Luga et al. 2012, Beckett et al. 2013), e.g. in Drosophila melanogaster EVs were supposed to be associated with Wnt signalling and in signal transduction (Beckett et al. 2013).

In addition to their physiological functions, EVs take part in multiple pathological processes, including cancer metastasis (Braicu et al. 2015) EVs may play a role in neurodegenerative diseases. It is known that several proteins which are related to neurodegenerative disease are released within EVs. For instance, prions (Fevrier et al. 2004b), $\beta$-amyloid peptide (Rajendran et al. 2006) and a-Syn (Emmanouilidou et al. 2010) and it is possible that EVs related to these proteins are involved in disease propagation via the interaction with recipient cells (Raposo et al. 2013). 


\subsection{SUMOylation}

SUMO (small ubiquitin like modifiers) proteins are ubiquitously expressed in all eukaryotic cells and can be conjugated to other proteins. SUMO modification is associated with regulation of gene transcription, cell cycle, DNA repair and protein localisation (Melchior 2000, Johnson 2004, Ulrich 2005).

\subsubsection{The family of small ubiquitin like modifiers SUMO}

Small ubiquitin like modifiers (SUMO-1 to SUMO-4) are a protein family that shares about $20 \%$ sequence homology to Ubiquitin. SUMO can be attached to lysine residues of various target proteins (Gareau et al. 2010). It is known that SUMO proteins are widely expressed in eukaryotic organisms. Interestingly, some lower organisms like yeast, $D$. melanogaster or $C$. elegans only encode one single SUMO gene (Geiss-Friedlander et al. 2007). In contrast, plants and vertebrates have several SUMO genes. The human genome encodes for several SUMO proteins (SUMO-1 to SUMO-4) (Melchior 2000, Guo et al. 2004).It has been shown that the SUMO proteins, SUMO-1 to SUMO-3 are widely expressed. Contrarily to SUMO-4, which has been shown to be mainly expressed in kidney, spleen and lymph nodes (Guo et al. 2004). The isoforms of SUMO-2 and SUMO-3 share a $97 \%$ sequence homology to each other, and $50 \%$ homology to SUMO-1. For all three isoforms different functions have been described (Saitoh et al. 2000, Rosas-Acosta et al. 2005, Vertegaal et al. 2006). In their conjugatable form SUMO-2 and SUMO-3 only differ in three aa residues in their N-terminus, therefore both isoforms are summarized to the subfamily SUMO-2/3 (Hay 2005). The physiological role of SUMO-4 is not uncovered till now, but it has been shown that SUMO-4 differs from the other SUMO-isoforms (Owerbach et al. 2005). Recently it has been shown that SUMO-4 is able to inhibit NFKB transcriptional activity (Hwang et al. 2012). In contrast to the other SUMO forms, SUMO-4 bears a proline residue in its C-terminus instead of a glutamine. Therefore, it seems that SUMO-4 is unable to form covalent isopeptide bonds with substrate proteins which prevent the maturation to a conjugatable form (Owerbach et al. 2005).

Interestingly, a flexible N-terminal stretch of $10-25$ aa is a common feature in all SUMO isoforms. This stretch is not found in other Ubiquitin-related proteins and is supposed to be essential for SUMO chain formation (Tatham et al. 2001). A large number of SUMO conjugation target proteins can act as transcription factors or act as other nuclear proteins which can be involved in gene expression or DNA integrity (Gareau et al. 2010). Changes in levels of SUMO conjugation to other proteins can therefore be expected to have a major impact on the fate of cells. 


\subsubsection{The SUMOylation pathway}

SUMOylation depends on the formation of an isopeptide bond between the C-terminal Glycin (Gly) residue of SUMO and the $\varepsilon$-amino group of a Lysine (Lys) residue in the target protein. SUMOylation as well as ubiquitination are dependent on an enzymatic cascade, which involves an E1-activating enzyme, an E2-conjugation enzyme and $f$ an E3-ligation enzyme (Fig. 5). Interestingly, SUMO-1 and SUMO-2/3 conjugation is driven by the identic enzymatic pathway (Tatham et al. 2001). The SUMOylation process is a reversible process, which primarily takes place at consensus motifs in the target proteins. This common consensus motif is defined as $\Psi-\mathrm{K}-\mathrm{X}-[\mathrm{D} / \mathrm{E}]$, at which $\Psi$ can be any large hydrophobic residue (I, $\mathrm{V}$ or $\mathrm{L}$ ), $\mathrm{K}$ is defined as the target lysine, $\mathrm{X}$ can be any residue and $\mathrm{D} / \mathrm{E}$ are aspartate or glutamate residues (Rodriguez et al. 2001, Sampson et al. 2001).

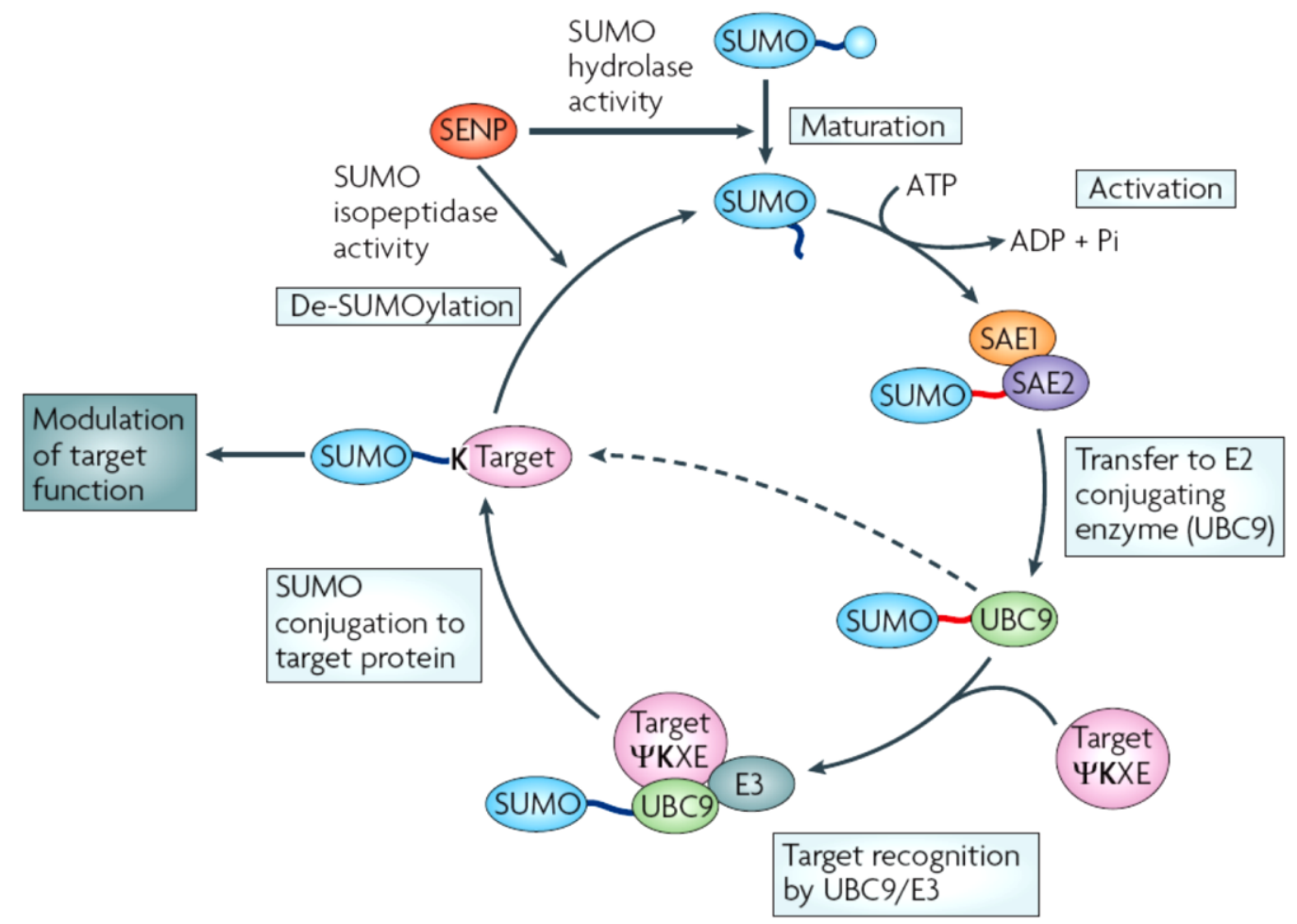

Fig. 5: Schematic representation of the SUMO cycle

SUMO-1 and SUMO-2/3 are first synthesized as precursors proteins and matured by specific SUMO proteases (SENPs), indicating the C-terminal Gly-Gly motif of SUMO. Subsequently SUMO is activated by the E1-activating enzyme, driven by the hydrolysis of ATP. Activated isoforms of SUMO are transferred to the E2-conjugating enzyme Ubc9. At the end of the cascade an isopeptide bond is formed between the $\varepsilon$-amino group of the acceptor lysine and the C-terminal carboxyl group of SUMO. Sumoylation can be reversed due to the activity of specific isopetidase of the SENP family. Adapted from (Martin et al. 2007). 
Briefly, SUMO precursor's proteins can be activated via the E1 activating enzyme (Desterro et al. 1999, Gong et al. 1999), via the hydrolysis of ATP.

Subsequent of this reaction, SUMO is transferred to the E2-activating enzyme, termed Ubc9 (Desterro et al. 1997, Johnson et al. 1997, Lee et al. 1998), which results in the formation of a thioester bond (Hay 2005) and finally in the conjugation of SUMO to the substrate, mediated by the E3-conjugating enzyme.

\subsubsection{Enzymes involved in the SUMOylation process}

The E1 activating and the E2 conjugating enzymes are identical in all SUMO paralogues and are also structurally comparable with the E1 and E2 enzymes involved in ubiquitination (Hochstrasser 2009). Enzyme Sae1 is known known to catalyse the formation of a thioester bond between Sae2 and the C-terminus of SUMO, which is driven by the hydrolysis of ATP (Lois et al. 2005). SUMO is now activated and can be transferred to Ubc9, the E2 enzyme in the SUMOylation process, which is driven by an intermolecular thiol transfer (Lois et al. 2005). Interestingly, the transfer of SUMO from Ubc9 to target proteins can also mediated via two ligase independent mechanisms. Firstly, Ubc9 can directly recognize the consensus motif $\Psi-K-X-[D / E]$. Secondly, SUMO target proteins can contain SUMO interacting motifs (SIM) (see section 1.5.2.2) to mediate the conjugation to SUMO on their own (Meulmeester et al. 2008, Zhu et al. 2008). Another group of enzymes that are involved in the SUMOylation process are so called SUMO ligases or E3 ligating enzymes. These enzymes are known to catalyse conjugation of SUMO to the lysine residue in the target protein (Wilkinson et al. 2010). It has been shown that there are three different groups of E3 ligases which can be involved in the SUMOylation pathway. So called SP-RING-finger like E3 ligases are known to function as adaptor proteins and are able to directly bind Ubc9 and the SUMO target protein (Johnson et al. 2001, Takahashi et al. 2001). In vertebrates these ligase are known as protein inhibitor of activated STAT (PIAS) (Hochstrasser 2001). In mammals five different PIAS proteins are discovered so far (Palvimo 2007). A second group of E3 ligases is defined as a nuclear pore protein and termed as Ran binding protein 2 (RanBP2) (Pichler et al. 2002). It has been shown that RanBP2 is able to raise the activity of Ubc9, but does not directly interact with the target protein (Reverter et al. 2005).

Another important group of enzymes which is involved in the SUMOylation process are sentrin specific proteases (SENPs). These enzymes are involved in the processing of SUMO peptides and in the de-conjugation process of SUMOylated target proteins (Hay 2007). In mammals six different SENPs enzymes are identified so far (Mukhopadhyay et al. 2007). 


\subsubsection{Non covalent SUMO binding mediated by SIM}

SUMO can also bind to other proteins non-covalently. This interaction is mediated by a short conserved SUMO-interaction motif (SIM) in the SUMO binding protein. The SIM motif is composed of a hydrophobic core, which is flanked $\mathrm{N}$-or $\mathrm{C}$ terminally by acidic residues or serine residues, respectively (Minty et al. 2000, Song et al. 2004, Hannich et al. 2005, Hecker et al. 2006). The SIM motif in SUMO-2 has been identified in a groove between the $\alpha$-helix and the $\beta$-sheet and includes amino acids Q30, F31 and I33 (Hecker et al. 2006, Sun et al. 2007). Mutations of these residues to alanines abrogate the interaction of SUMO-2 with SIM domains (Meulmeester et al. 2008, Zhu et al. 2008).

\subsubsection{SUMOylation in neurodegenerative diseases}

SUMO modification has been suggested to be involved in a variety of neurodegenerative disease (Dorval et al. 2007a). In the last decades it becomes more and more evident that SUMOylation plays an important role and is associated with different neurological disorders, like PD, $A D$ and Huntington's disease (HD).

\subsubsection{SUMOylation in Alzheimer's disease}

$A D$ is the most common age related neurodegenerative disorder and is characterised by extracellular plaques composed of $a \beta$ and neurofibrillary tangles, consisting of hyperphosphorylated tau (Wilkinson et al. 2010). It has been shown that both proteins are potential substrates for SUMOylation (Gocke et al. 2005, Dorval et al. 2006, Zhang et al. 2008). Li and co-workers observed that SUMO-2 decreases the a $\beta$ production (Li et al. 2003). In contrast, Dorval and co-workers showed increased a generation upon SUMO-3 overexpression which is independent of SUMO conjugation and might be mediated by indirect effect of SUMO-3 on APP and BACE expression levels (Dorval et al. 2007b). For the protein Tau, another key-player in $A D$, it has been shown that this protein can be SUMOylated by SUMO1 at aa Lys 340. (Dorval et al. 2006). Importantly, SUMOylation at K340 inhibits tau degradation through deregulation of tau phosphorylation and ubiquitination, thereby facilitating its assembly into fibrils (Luo et al. 2014) 


\subsubsection{SUMOylation in Huntington's disease}

The best characterised polyQ disorder is Huntington's disease (HD). This disease is caused by the expansion of a polyQ repeat in the $\mathrm{N}$-terminus of the Huntingtin ( $\mathrm{Htt}$ ) protein (Gil et al. 2008). PolyQ disorders are dominantly inherited disorders with variations in the age of onset of the disease, which is depends on the lengths of polyQ repeats (Walker 2007). It has been shown that a pathogenic fragment of $\mathrm{Htt}$ can be SUMOylated by SUMO-1 and as well as is ubiquitinated at the lysine residue in the N-terminus of the Htt protein (Steffan et al. 2004). SUMOylation stabilizes the pathogenic fragment of $\mathrm{Htt}$ (Httex1p) and is able to reduce its ability to form aggregates. In a $D$. melanogaster disease model of HD SUMOylation of the pathogenic Htt fragment increases neurodegeneration, contrarily to ubiquitination that has been shown to decrease neurotoxicity (Steffan et al. 2004). In a transgenic Drosophila model which is expressing both SUMO deficient and ubiquitination deficient mutations of $\mathrm{Htt}$, a reduced Htt toxicity has been observed. These findings indicate that SUMOylation and ubiquitination of $\mathrm{Htt}$ are involved in the stabilising of toxic $\mathrm{Htt}$ species and that the balance between both modifications is disturbed in HD (Steffan et al. 2004).

\subsubsection{SUMOylation in Parkinson's disease}

In PD a-Syn is preferentially SUMOylated by SUMO-1 and to a lesser extent by SUMO-2/3 (Dorval et al. 2006). The influence of SUMOylation on $\alpha$-Syn aggregation and toxicity, especially the formation of fibrils, under in vitro conditions has been shown by Krumova and co-workers (Krumova et al. 2011). They have shown by several approaches that SUMOylation of $\alpha$-Syn inhibits neurotoxic fibril formation of $\alpha$-Syn (Krumova et al. 2011). In addition, SUMOylation-deficient mutants of $\alpha$-Syn showed a higher toxicity in mouse models, compared to wt $\alpha$-Syn (Krumova et al. 2011). SUMO-1 has also been shown to be a component of Lewy bodies in brain tissue of patients with DLB and MSA (Pountney et al. 2005). Additionally, Parkin non-covalently interacts with SUMO-1, in vitro and in vivo (Um et al. 2006). This interaction results in the auto-ubiquitination and in the nuclear localisation of Parkin (Um et al. 2006). In addition SUMOylation of the protein DJ-1 has also been described (Shinbo et al. 2006). DJ-1 is known as a regulator for the expression of several genes which are linked to the cellular response to oxidative stress conditions (Taira et al. 2004). Oxidative stress conditions are known to induce the loss of dopaminergic neurons in PD (Jenner 2003). 


\section{SUMOylation of $\alpha$-Synuclein}

By mass spectrometry of SUMOylated a-Syn Krumova et al identified eleven lysine residues of $\alpha$-Syn which serve as SUMO acceptor sites (Krumova et al. 2011). Nevertheless, only two lysine residues $\mathrm{K} 96$ and $\mathrm{K} 102$ are counting for more than $50 \%$ of the $\alpha$-Syn SUMOylation. Mutations of these lysine residues (K96R K102R) impairs SUMOylation to the same extent as a D98A E104A double mutation, which disrupts the consensus sequence for the recognition of adjacent SUMO receptor lysines. These finding are consistent with the observations of Sapetschnig and colleague. They showed that SUMO acceptor sites requires the acidic residues for efficient SUMOylation (Sapetschnig et al. 2002).

Furthermore, Krumova et al. found that the ubiquitination status of $\alpha$-Syn is unaffected by mutated K96 and K102 (Krumova et al. 2011) 


\section{Materials and Methods}

\subsection{Materials}

\subsubsection{Chemicals and Consumables}

Unless stated otherwise, all chemicals which were used in this study were purchased from AppliChem (Darmstadt, Germany), Merck KGaA (Darmstadt, Germany) or Sigma Aldrich Chemie GmbH (Munich, Germany). Cell culture media, supplements, sera and antibiotics were purchased from PAA Laboratories $\mathrm{GmbH}$ (Pasching, Austria), GE Healthcare (Chalfont Buckinghamshire, UK) and Gibco ${ }^{\circledR}$ by Life Technologies (Darmstadt, Germany). Consumables which were used in cell culture, molecular biology and biochemistry analysis were purchased from Starlab GmbH (Hamburg, Germany), Eppendorf AG (Hamburg, Germany), Greiner Bio-One GmbH (Frickenhausen, Germany) or Falcon (Becton Dickinson Labware Europe, Le Pont de Claix, France).

\subsubsection{Cell lines and primary cells}

\subsubsection{1. $\quad \underline{\text { Cell lines }}$}

Cell lines used in this study are specified in Table 1.

Table 1: Cell lines

\begin{tabular}{l|l|l}
\hline \hline Name & Cell type & Obtained from \\
\hline \hline N2a & Mouse neuroblastoma cell line & (Schubert et al. 1969) \\
Oli-neu & Mouse oligodendrocyte precursor cell & J. Trotter, University of Mainz, \\
& line & Germany \\
HEK & Human embryonic kidney cell line & (Graham et al. 1973a, Graham et \\
& (HEK 293) & al. 1973b, van der Eb 1973) \\
E. coli $(\mathbf{D H} \mathbf{\alpha})$ & chemically competent E.coli strain & Invitrogen Darmstadt, Germany \\
\hline \hline
\end{tabular}

\subsubsection{2. $\quad$ Primary cells}

Primary cortical neurons were prepared from E16.5 NMRI mouse embryos (for details see section 2.2.2.1). 


\subsubsection{Antibodies}

Primary antibodies that were used in this study are specified in Table 2. Secondary fuorophore- or horseradish peroxidase (HRP) conjugated were purchase from Invitrogen (Darmstadt, Germany) and Dianova (Hamburg, Germany).

Table 2: Primary Antibodies

\begin{tabular}{l|l|l|l}
\hline \hline Antibody & Host species & Application & Obtained from \\
\hline \hline Myc (clone 9E10) & Mouse & WB & Sigma-Aldrich St. Louis, MO, USA \\
Myc (clone 9B11) & Mouse & WB, IF & Cell Signaling Danvers, MA, USA \\
Flotillin-2 & Mouse & WB & BD Biosciences Heidelberg, Germany \\
a-Synuclein & Mouse & WB, IF & Invitrogen Darmstadt, Germany \\
Alix/AIP1 & Mouse & WB & BD Biosciences Heidelberg, Germany \\
Alix(clone 3A9) & Mouse & WB & GeneTex Inc., Irvine, CA, USA \\
TSG-101 & Mouse & WB & Santa Cruz Dallas, TX, USA \\
CD63 & Mouse & WB & BD Biosciences Heidelberg, Germany \\
Beta Amyloid & Mouse & WB & Covance Inc., Princeton, NJ, USA \\
(6E10) & & WB & Chemicon Limburg, Germany \\
GluR 2/3 & Rabbit & WB & Chemicon (Limburg, Germany) \\
GluR 1 & Rabbit & WB & Sigma-Aldrich St. Louis, MO, USA \\
Calnexin & Rabitt & WB, IF & Invitrogen Darmstadt, Germany \\
GFP & Rabitt & WB & Santa Cruz Biotechnology Inc., Dallas, \\
Ubc9 & Rabbit & & TX, USA \\
\hline & &
\end{tabular}

\subsubsection{Nucleotide constructs}

\subsubsection{Previously published plasmids}

Plasmids which were kindly provided by other laboratories are listed in Table 3.

Table 3: Previously published plasmids

\begin{tabular}{|c|c|}
\hline Plasmid & Obtained from \\
\hline $\begin{array}{l}\text { pEYFP-N1 } \\
\text { Rab5Q79L GFP } \\
\text { pcDNA3.1- } \Delta N-\alpha-\text { Synuclein } \\
\text { pcDNA3.1-Myc-SUMO-2 } \\
\text { pcDNA3.1-Myc-SUMO-2 } \Delta G G^{1} \\
\text { pEYFP-SUMO-1 } \\
\text { pEYFP-SUMO-1 } \Delta \text { GG }^{1} \\
\text { pcDNA3.1- Myc- } \alpha \text {-Synuclein } \\
\text { pcDNA3.1-Myc- } \alpha \text {-Synuclein } 2 \mathrm{KR}^{2} \\
\text { pcDNA3.1-Myc- } \alpha \text {-Synuclein } 2 \mathrm{AA}^{3} \\
\text { pTE1E2S1 } \\
\text { pT7.7 } \\
\alpha-S y n u c l e i n \text { phGLuc1 (S1) } \\
\alpha-S y n u c l e i n \text { phGLuc2 (S2) } \\
\text { pcDNA3.1-Myc- } \alpha-S y n u c l e i n-S U M O-2 \Delta G G \\
\text { pcDNA3.1-GFP-SUMO-2 } \Delta G G \\
\text { pcDNA3.1-Myc-SUMO-2 } \Delta G G \text { cleft } \\
\text { pcDNA3.1-Myc-SUMO-2 } \Delta G G \text { cleft+loop }\end{array}$ & $\begin{array}{l}\text { Clonetech, Mountain View, CA, USA } \\
\text { M. Zerial, MPI-CBG, Dresden, Germany } \\
\text { (Karube et al. 2008) } \\
\text { (Krumova et al. 2011) } \\
\text { (Krumova et al. 2011) } \\
\text { (Krumova et al. 2011) } \\
\text { (Krumova et al. 2011) } \\
\text { (Krumova et al. 2011) } \\
\text { (Krumova et al. 2011) } \\
\text { (Krumova et al. 2011) } \\
\text { (Uchimura et al. 2004) } \\
\text { P. Lansbury Cambridge, MA, USA } \\
\text { (Outeiro et al. 2008) } \\
\text { (Outeiro et al. 2008) } \\
\text { K. Eckermann Neurology UMG Göttingen, Germany } \\
\text { K. Eckermann Neurology UMG Göttingen, Germany } \\
\text { K. Eckermann Neurology UMG Göttingen, Germany } \\
\text { K. Eckermann Neurology UMG Göttingen, Germany }\end{array}$ \\
\hline
\end{tabular}


pcDNA3.1-Ubiquitin-SUMO-2 $\triangle \mathrm{GG}$ pR4-PLP-Myc

pcDNA3.1-MLV Gag-GFP

GFP-VPS4dn (E233Q)

GFP-VPS4

pShuttleCMV YFP-APPsw

YFP-APPsw-SUMO-2 $\triangle \mathrm{GG}$
K. Eckermann Neurology UMG Göttingen, Germany

J. Trotter, University of Mainz, Germany

W. Mothes, Yale University New haven, CT, USA

P. Woodman, University of Manchester, UK

P. Woodman, University of Manchester, UK

P. Keller, MPI-CBG Dresden, Germany

K. Eckermann Neurology UMG Göttingen, Germany

${ }^{1} \mathrm{C}$-terminal deletion mutant that cannot be conjugated

${ }^{2}$ bearing the double mutation K96R K102R

${ }^{3}$ bearing the double mutation D98A E104A

${ }^{4}$ bearing following mutations Q30A F31A K32A I33A L42A Y46A

${ }^{5}$ bearing following mutations H16A Q30A F31A K32A I33A H36A L42A Y46A D62A

SUMO-2-luciferase construct (SUMO-2-S3) was created by cloning the amino-terminal fragment of humanized Gaussia Luciferase including the same linker as used in S2 into BamHI/EcoRI sites of pcDNA3. SUMO-2 was subsequently subcloned into EcoRI/Xhol sites.

\subsubsection{Self constructed plasmids}

PcDNA3.1-GFP-SUMO-2 $\triangle G G \Delta S I M$ was generated by site directed mutagenesis to introduce the triple amino acid point mutation Q30A F31A I33A. Mutagenesis was performed according to the manufactures protocol (Quick Change Site-Directed Mutagenesis Kit, Agilent Technologies, Waldbronn, Germany).

\subsubsection{3. $\quad$ siRNA constructs}

To down regulate expression of Alix/AIP1 or TSG-101, the following siRNAs were used as specified in Table 4.

Table 4: siRNA constructs

\begin{tabular}{l|l|l}
\hline \hline Target & Target sequence 5'-3' & Reference \\
\hline \hline Alix mouse & AAGAACCTGGATAATTGATGAA & Qiagen GmbH, Hilden, Germany \\
TSG-101 mouse & CACTGTATAAACAGATTCTAA & Qiagen GmbH, Hilden, Germany \\
Ubc9 human (UBE2I) & CAAAAAAUCCCGAUGGCACUU & GE Dhamacon, Lafayette, CO, USA \\
\hline \hline
\end{tabular}




\subsubsection{Phospholipids}

Phospholipids which were used in this study are specified in Table 5.

Table 5: Phospholipids

\begin{tabular}{|c|c|c|}
\hline "Lipid & structure & Reference \\
\hline POPS & & $\begin{array}{l}\text { Avanti Polar Lipids } \\
\text { Alabaster; AL, USA }\end{array}$ \\
\hline POPC & & $\begin{array}{l}\text { Avanti Polar Lipids } \\
\text { Alabaster; AL, US) }\end{array}$ \\
\hline $\mathrm{PI}(3) \mathrm{P}$ & & $\begin{array}{l}\text { Avanti Polar Lipids } \\
\text { Alabaster; AL, US) }\end{array}$ \\
\hline $\mathrm{PI}(5) \mathrm{P}$ & & $\begin{array}{l}\text { Avanti Polar Lipids } \\
\text { Alabaster; AL, USA }\end{array}$ \\
\hline $\mathrm{PI}(3,5) \mathrm{P}_{2}$ & & $\begin{array}{l}\text { Avanti Polar Lipids } \\
\text { Alabaster; AL, USA }\end{array}$ \\
\hline $\mathrm{PI}(4,5) \mathrm{P}_{2}$ & & $\begin{array}{l}\text { Avanti Polar Lipids } \\
\text { Alabaster; AL, USA }\end{array}$ \\
\hline $\mathrm{PI}(3,4,5) \mathrm{P}_{3}$ & & $\begin{array}{l}\text { Avanti Polar Lipids } \\
\text { Alabaster; AL, USA }\end{array}$ \\
\hline
\end{tabular}




\subsubsection{Buffer and Solutions}

\subsubsection{1. $\quad$ Phosphate buffered saline (PBS)}

PBS was prepared according to (Sambrook et al. 2001).

10x PBS (1 L)

$80.0 \mathrm{~g} \mathrm{NaCl}$

$2.0 \mathrm{~g} \mathrm{KCl}$

$14.4 \mathrm{~g} \quad \mathrm{Na}_{2} \mathrm{HPO}_{4}$

$2.4 \mathrm{~g} \quad \mathrm{KH}_{2} \mathrm{PO}_{4}$

To obtain 1x PBS, 10x PBS was diluted 10 times with bi-distilled $\mathrm{H}_{2} \mathrm{O}$ and the $\mathrm{pH}$ was adjusted to 7.4 .

\subsubsection{Tris buffered saline (TBS)}

TBS was prepared according to (Sambrook et al. 2001)

$$
\begin{array}{rl}
10 x & \text { TBS }(1 \mathrm{~L}) \\
80.0 \mathrm{~g} & \mathrm{NaCl} \\
2.0 \mathrm{~g} & \mathrm{KCl} \\
30.0 \mathrm{~g} & \text { Tris }
\end{array}
$$

To obtain $1 x$ TBS, 10x TBS was diluted 10 times with bi- distilled $\mathrm{H}_{2} \mathrm{O}$ and the $\mathrm{pH}$ was adjusted to 7.4 .

\subsubsection{HEPES/sucrose stock solution}

HEPES/sucrose stock solution was prepared according to (Théry et al. 2006).

\section{1x HEPES/sucrose ( $1 \mathrm{~L}$ )}

$4.8 \mathrm{~g}$ Hydroxyethylpiperazine-N-2-ethanesulfonic acid (HEPES), (^20 mM)

$856 \mathrm{~g}$ Protease-free sucrose, $(\bumpeq 2.5 \mathrm{M})$

The $\mathrm{pH}$ was adjusted to 7.4 . 


\title{
2.1.6.4. $\quad$ Homogenisation-buffer (HB)
}

$\begin{aligned} 320 \mathrm{mM} & \text { Sucrose } \\ 1 \mathrm{mM} & \text { EDTA (Ethylenediaminetetraacetic acid) } \\ 20 \mathrm{mM} & \text { HEPES }\end{aligned}$

The $\mathrm{pH}$ was adjusted to 7.0 and Complete protease inhibitor (Roche Diagnostics $\mathrm{GmbH}$, Mannheim, Germany) was added according to the manufactures protocol.

\subsubsection{5. $\quad$ CHAPS lysis buffer}

\section{$1 \mathrm{x}$ lysis buffer $(1 \mathrm{~L})$}

$10 \mathrm{~g}$ 3-[(3-cholamidopropyl)dimethylammonio]-1-propanesulfonate (CHAPS), (1\%)

$6.1 \mathrm{~g}$ Tris(hydroxymethyl)aminomethane (Tris), $(\bumpeq 50 \mathrm{mM})$

$1.5 \mathrm{~g} \quad \mathrm{EDTA},(\bumpeq 5 \mathrm{mM})$

The $\mathrm{pH}$ was adjusted to 8.0.

\subsubsection{Protein loading buffer}

\author{
5x loading buffer \\ $10 \%$ Glycerol \\ $50 \mathrm{mM}$ Tris- $\mathrm{HCl}$ \\ $2 \mathrm{mM}$ EDTA \\ $2 \%$ SDS \\ $144 \mathrm{mM} \quad \beta$-Mercaptoethanol \\ $0.05 \%$ Bromophenol blue
}

The $\mathrm{pH}$ was adjusted to 6.8 . 


\subsubsection{7. $\quad$ Resolving gel buffer}

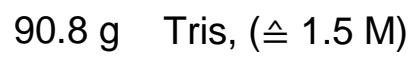

The $\mathrm{pH}$ was adjusted with $\mathrm{HCl}$ to 8.8 .

\subsubsection{Stacking gel buffer}

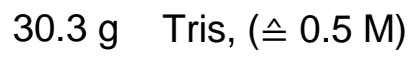

The $\mathrm{pH}$ was adjusted with $\mathrm{HCl}$ to 6.8 .

\subsubsection{9. $\quad$ 10x Running buffer}

$143 \mathrm{~g}$ Glycine

$30.3 \mathrm{~g}$ Tris

$10 \mathrm{~g}$ Sodium dodecyl sulfate (SDS)

To achieve $1 x$ running buffer, 10x running buffer was diluted 10 times with bi-distilled $\mathrm{H}_{2} \mathrm{O}$.

\subsubsection{0. $\quad \underline{10 x \text { Transfer buffer }}$}

$143 \mathrm{~g}$ Glycine

$30.3 \mathrm{~g}$ Tris

\subsubsection{1. $\quad$ 10x Transfer buffer}

$100 \mathrm{~mL} \quad 10 x$ Transfer buffer

$200 \mathrm{~mL}$ Methanol

To achieve the final volume $1 \mathrm{~L}, 1 \mathrm{x}$ transfer buffer was filled up with bi-distilled $\mathrm{H}_{2} \mathrm{O}$. 


\title{
2.1.7. Media and sera
}

\subsubsection{1. $\quad$ Commercial media}

Table 6 illustrates commercially available media, sera and additives used in this study.

Table 6: Commercial media and solutions

\begin{tabular}{|c|c|}
\hline Medium/Solution & Obtained from \\
\hline $\begin{array}{l}\text { Dulbecco's Modified Eagle Medium (DMEM) } \\
\text { Fetal Calf Serum (FCS) } \\
\text { GlutaMAX'TM-I supplement } \\
\text { Hanks' Balanced Salt Solution (HBSS) } \\
\text { Opti-MEM + GlutaMAX } \text { TM }-I^{\text {TM }} \\
\text { Penicillin/Streptomycin (Pen/Strep) 100x } \\
\text { The TransIT@-LT1 Reagent } \\
\text { Phosphate Buffered Saline (PBS) } \\
\text { LB medium + LB agar plate } \\
\text { B27-Supplement 50x } \\
0.25 \% \text { Trypsin-EDTA 1x } \\
0.05 \% \text { Trypsin-EDTA 1x } \\
\text { Oligofectamine }{ }^{\mathrm{TM}} \text { Reagent } \\
\text { MEM 10x } \\
\text { Sodium Pyruvate } 100 \text { mM, 100x } \\
\text { Sodium Bicarbonate Solution 7.5\% } \\
\end{array}$ & 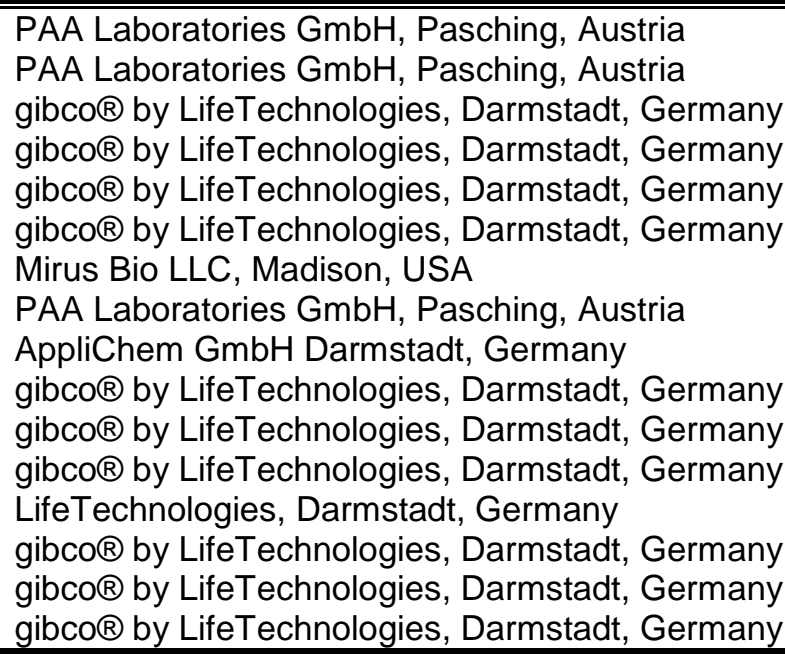 \\
\hline
\end{tabular}

\subsubsection{General growth medium}

General growth medium was used in this study to cultivate mouse neuroblastoma cells (N2a) and human embryonic kidney cells (HEK 293). For preparation of serum free medium, no fetal calf serum was added.

\section{General growth medium (500 ml)}

\author{
$5 \mathrm{~mL}$ GlutaMAX ${ }^{\mathrm{TM}}-$ I supplement, $200 \mathrm{mM}$ \\ $5 \mathrm{~mL}$ Penicillin/Streptomycin (Pen/Strep), $5000 \mathrm{U} / 5000 \mu \mathrm{g}$ \\ $50 \mathrm{~mL}$ Fetal calf serum
}

in Dulbecco's Modified Eagle Medium (DMEM) with $4.5 \mathrm{~g} / \mathrm{L}$ glucose. 


\subsubsection{3. $\quad \underline{\text { SATO-medium }}$}

SATO-medium was used to cultivate Oli-neu cell line. For preparation of serum free medium, no horse serum was added.

\section{SATO-medium (100 ml)}

$1 \mathrm{~mL}$ Insulin-Transferrin-Selenium-A Supplement ITS-A, 100x

$1 \mathrm{~mL}$ Putrescine dihydrochloride, stock $10 \mathrm{mM}$ in DMEM

$10 \mu \mathrm{L}$ Progesterone, stock $2 \mathrm{mM}$ in ethanol

$10 \mu \mathrm{L}$ Triiodothyronine (Calbiochem/Merck KGaA, Darmstadt, Germany), $5 \mathrm{mM}$ stock in ethanol

$13 \mu \mathrm{L}$ L-Thyroxine (Calbiochem/Merck KGaA, Darmstadt, Germany), $4 \mathrm{mM}$ stock in $0.26 \mathrm{~N} \mathrm{NaOH}, 25 \%$ ethanol

$1 \mathrm{~mL}$ GlutaMAX ${ }^{\mathrm{TM}}-$ I supplement, $200 \mathrm{mM}$

$1 \mathrm{~mL}$ Penicillin/Streptomycin, $5000 \mathrm{U} / 5000 \mu \mathrm{g}$

$5 \mathrm{~mL}$ Horse serum

in Dulbecco's Modified Eagle Medium (DMEM) with $4.5 \mathrm{~g} / \mathrm{L}$ glucose.

\subsubsection{4. $\quad$ MEM-B27}

MEM-B27 was used in this study to cultivate primary cortical neurons from E16.5 NMRI mouse embryos.

\section{MEM-B27 (250 ml)}

$15 \mathrm{~mL}$ 10x MEM

$7.25 \mathrm{~mL}$ Sodium- Bicarbonate

$7.5 \mathrm{~mL} \quad 20 \%$ Glucose

$25 \mathrm{~mL}$ Sodium-Pyruvate

2,5 mL Penicillin/Streptomycin (Pen/Strep), $5000 \mathrm{U} / 5000 \mu \mathrm{g}$

$2,5 \mathrm{~mL}$ GlutaMAX ${ }^{\mathrm{TM}}-\mathrm{I}$ supplement, $200 \mathrm{mM}$

$5 \mathrm{~mL}$ B27-Supplement

The final volume of $250 \mathrm{~mL}$ was adjusted with bi-distilled $\mathrm{H}_{2} \mathrm{O}$. 


\subsubsection{Commercial kits}

Commercial Kits that were used in this study are listed in Table 7.

Table 7: Commercial Kits

\begin{tabular}{l|l|l}
\hline \hline Kit & Application & Obtained from \\
\hline \hline NucleoBond Xtra Midi Kit & Plasmid DNA-Isolation & Machery-Nagel, Düren, Germany \\
$\begin{array}{l}\text { Quick Change Site- } \\
\text { Directed Mutagenesis }\end{array}$ & Point mutagenesis & $\begin{array}{l}\text { Agilent Technologies, Waldbronn, } \\
\text { Germany }\end{array}$ \\
\hline \hline
\end{tabular}

\subsubsection{Software}

Software that was used in this study is specified in Table 8.

Table 8: Software

\begin{tabular}{l|l|l}
\hline \hline Software & Application & Source \\
\hline \hline ImageJ & Image processing and analysis & http://rsbweb.nih.gov/ij/ \\
MS Office Exel 2007 & Spreadsheet analysis & Microsoft GmbH, Berlin, Germany \\
MS Office Word 2007 & Text processing & Microsoft GmbH, Berlin, Germany \\
EndNote X5 & Citization/Bibliographie & Thomson Reuters, New York City, \\
& & NY, USA \\
Leica Confocal Software, & Acquisition of confocal images & Leica Microsystems, Mannheim, \\
2.61 & Germany \\
NanoSight Tracking & Tracking and analysis of EVs & NanoSight, Amesbury, UK \\
Analysis Software 2.3 & & \\
\hline \hline
\end{tabular}




\subsection{Methods}

\subsubsection{Molecular biology methods}

\subsubsection{1. $\quad$ Site-directed mutagenesis}

To introduce amino acid point mutations into the SIM domain of the SUMO-2 protein, sitedirected mutagenesis was performed using Stratagene QuickChange ${ }^{\mathrm{TM}}$ Site-Directed Mutagenesis Kit (see Table 7). This method is based on site- directed mutagenesis using double stranded DNA templates (Braman et al. 1996).

\subsubsection{2. $\quad \underline{\text { Transformation of Escherichia coli (E. coli) }}$}

To amplify plasmid DNA constructs in bacteria, the chemo-competent $E$. coli strand "Library efficiency ${ }^{\circledR} \mathrm{DH} 5 \mathrm{a}^{\mathrm{TM}}$ " (Table 1) was used. For transformation $45 \mu \mathrm{L}$ of competent cells were thawed for $10 \mathrm{~min}$ on ice. Afterwards, $100 \mathrm{ng}$ from the plasmid DNA of interest were added to the cells and incubated on ice for $30 \mathrm{~min}$ followed by a heat shock at $45^{\circ} \mathrm{C}$ for $42 \mathrm{~s}$. After recovery on ice for $2 \mathrm{~min}, 600 \mu \mathrm{L}$ of S.O.C medium was added and the cells were incubated for $1 \mathrm{~h}$ at $37^{\circ} \mathrm{C}$ with agitation. In order to select single transformants, cells were plated on LB- Agar plates with the appropriate antibiotics (100 $\mu \mathrm{g} / \mathrm{ml}$ ampicillin or $50 \mu \mathrm{g} / \mathrm{ml}$ kanamycin) and incubated over night at $37^{\circ} \mathrm{C}$.

\subsubsection{Plasmid DNA isolation from E. coli}

To enlarge the amount of plasmid DNA from transformed $E$. coli at a medium scale, a single colony was picked from a LB-Agar plate and transferred to $150 \mathrm{~mL}$ of antibiotics supplemented LB medium. The culture was incubated for $16-20 \mathrm{~h}$ at $37^{\circ} \mathrm{C}$ with $200 \mathrm{rpm}$.

Afterwards, cells were harvested by centrifugation for $10 \mathrm{~min}$ at $6000 \mathrm{rpm}$ and $4^{\circ} \mathrm{C}$.

Plasmid DNA was isolated with the NucleoBond Xtra Midi Kit (see Table 7), according to the manufactures protocol.

\subsubsection{Determination of DNA concentration}

The concentration of plasmid DNA in the final solution was measured by a NanoDrop 2000 Spectrophotometer (PEQLAB Biotechnologie GMBH, Erlangen, Germany). 


\subsubsection{Expression and purification of sumoylated a-Synuclein}

The expression and purification of human sumoylated wild-type $\alpha$-Syn was previously described (Krumova et al. 2011). Briefly, BL21 competent E.coli cells were co-transformed with the tricistronic plasmid pTE1E2S1, which codes for the expression of SUMO-1 and the E1 and E2 enzymes of the SUMOylation pathway (Uchimura et al. 2004), and the pT7.7 encoding for human wild-type $\alpha$-Syn (courtesy of the P. Lansbury laboratory, Harvard Medical School, Cambridge, MA). After enzymatic degradation of DNA, the bacterial extracts were heat precipitated at $95{ }^{\circ} \mathrm{C}$ for $10 \mathrm{~min}$ and the supernatant was subjected to column chromatography (GE Healthcare Äkta system) with a sequence of 3 columns: Q Shepharose fast flow, HiLoad 26/600 Superdex 200, and Mono Q 4.6/100 PE. Fractions of sumoylated a-Synuclein were combined and concentrated with an Amicon Ultracel Filter (10 kDa, Millipore), and purity assessed by polyacrylamide gel electrophoresis (PAGE) and electrospray ionization mass spectroscopy (ESI-MS). The protein concentration was estimated using a molar extinction coefficient at $280 \mathrm{~nm}$ of $9080 \mathrm{M}^{-1} \cdot \mathrm{cm}^{-1}$.

\subsubsection{Expression of recombinant SUMO-2 for NMR}

SUMO-2 was cloned into pET11 vector and expressed as previously described (Pichler et al. 2002). For $\mathrm{N}^{15}$ labelling of SUMO-2 proteins, bacterial cells were grown in $1 \mathrm{~L} L B$ at $37^{\circ} \mathrm{C}$ until the culture reached an optic density (OD600) of 0.6. Bacteria cultures were then centrifuged and resuspended in $500 \mathrm{ml}$ standard Minimal M9 media containing $3 \mathrm{~g}$ glucose. After 30 min incubation, $1 \mathrm{~g} \mathrm{~N}^{15} \mathrm{H}_{4} \mathrm{Cl}$ was added to the medium, Cells were frown for $1 \mathrm{~h}$ at $37^{\circ} \mathrm{C}$, before induction with $1 \mathrm{mM}$ IPTG. SUMO purification was performed as described, except that for gel-filtration analysis a buffer containing $20 \mathrm{mM} \mathrm{NaH}_{2} \mathrm{PO}_{4} / \mathrm{Na}_{2} \mathrm{HPO}_{4} \mathrm{pH} 6.8$, 100 mM KCl, 2 mM DTT was used. 


\subsubsection{Cell culture}

All cell culture work was carried out according to security level S1 safety rules. Work was executed under sterile conditions, involving antiseptic cleaning of the equipment with $70 \%$ ethanol, UV- treatment and sterile filtration of all media and solutions with a $0.22 \mu \mathrm{m}$ polyethersulfone (PES) filter (Corning Inc., Corning, NY, USA).

\subsubsection{Growth and maintenance of cells}

In general, cells were grown at $37^{\circ} \mathrm{C}$ and $5.0 \%$ (7.5\% for primary neurons) $\mathrm{CO}_{2}$ in humidified incubators. Specific cultivation procedures are described below. For collection of EVs cells were cultured in $10 \mathrm{~cm}$ plastic dishes, with general growth medium in the absence of serum.

\section{Oli-neu cell line}

The oligodendrocyte precursor cell line Oli-neu was grown in SATO medium (see section 2.1.7.3). The cells were grown on $75 \mathrm{~cm}^{2}$ cell culture flasks or $10 \mathrm{~cm}$ petri dishes which were previously coated with poly-L-lysine (PLL, $30 \mathrm{mg} / \mathrm{L}$, Sigma, St. Louis, MO, USA) for at least 30 min or overnight. Thereafter, dishes were washed 3 times with PBS. For passaging of Olineu cells was executed 1:6 every 2-3 days after a confluence of $70-90 \%$ was reached. For passaging, cells were washed off with cultured medium to bring them in suspension.

\section{Mouse neuroblastoma (N2a) and human embryonic kidney (HEK293) cell lines}

The N2a and HEK293 cell lines were grown in general growth medium (see section 2.1.7.2). These cells were grown on $75 \mathrm{~cm}^{2}$ cell culture flasks, $10 \mathrm{~cm}$ petri dishes, 6-well plates or on coverslips which were coated with PLL. Passaging of both cell lines were conducted 1:4 every second day after a confluence of $80-90 \%$ was reached. For passaging, cells were washed once with PBS and trypsinized with $3 \mathrm{~mL}$ of $0.25 \%$ trypsin/EDTA (see Table 6) for approximately $3 \mathrm{~min}$ until cells were detached. Afterwards, $10 \mathrm{~mL}$ of general growth medium was added to inhibit trypsin activity. The cells were used up to a passage 30 .

\section{Mouse primary cortical neurons}

Primary cortical neurons were prepared from E16.5 NMRI mouse embryos and cultured on PLL coated coverslips or petri dishes, in serum free MEM-B27. 


\subsubsection{2. $\quad$ Cryoconservation of cells}

\section{Freezing of cells}

To store cells for a long term period, cells were cultured on a $75 \mathrm{~cm}^{2}$ cell culture flask, to a confluence of $80-90 \%$. Cells were then cultured as described above (see section 2.2.2.1). Afterwards, the cell suspension was centrifuged at $900 \mathrm{rpm}$ for $10 \mathrm{~min}$ and the pellet was resuspended in $1.6 \mathrm{~mL}$ freezing medium (50\% FCS and 10\% DMSO in DMEM). Thereafter, the suspension was mixed gently and transferred into a Nalgene® sterile Cryogenic vial (Thermo Fisher Scienctific,Waltham, MA, USA). The vials were transferred into a Nalgene® Cryo $1^{\circ} \mathrm{C}$ Freezing Container (Thermo Fisher Scienctific,Waltham, MA, USA) which allows a slow freezing at a temperature dropping point of $1^{\circ} \mathrm{C} / \mathrm{min}$ in a $-80^{\circ} \mathrm{C}$ freezer. For permanent storage, cells were stored in liquid nitrogen.

\section{Thawing of cells}

Cryoconservated cells were taken out of liquid nitrogen and immediately incubated in a $37^{\circ} \mathrm{C}$ water bath for thawing. Rapidly after the medium was defrosted the cell suspension was carefully and slowly resuspended. Then the suspension was transferred into $10 \mathrm{~mL}$ prewarmed general growth medium. Afterwards the suspension was centrifuged at $900 \mathrm{rpm}$ for $10 \mathrm{~min}$ and the pellet was resuspended in pre-warmed medium and plated in a $75 \mathrm{~cm}^{2}$ cell culture flask. Growing cells were further passaged according to their cell type as described in section 2.2.2.1.

\subsubsection{3. $\quad$ Transfection of plasmids}

Introduction of plasmid DNA to mammalian cell lines was done via TransIT®- LT1 (Mirus Bio LLC, Madison, USA). At the time of transfection the cells were grown to a confluence of $70-80 \%$. The plasmid DNA and the transfection reagent were added to $600 \mu \mathrm{L}$ Opti- MEM (see Table 6), mixed gently and incubated at room temperature for 30-45 min.

Based on various vessel sizes, the transfection protocol used in this study was specified in Table 9. After incubation, the mixture was added drop wise to the cells, the vessel was shaken gently and the cells were kept under cultivation conditions for 8-12 h.

Table 9: Transfection protocols

\begin{tabular}{c|c|c|c}
\hline \hline Reagent & 12 well plate & 6 cm dish & 10 cm dish \\
\hline \hline Opti-Mem & $100 \mu \mathrm{L}$ & $300 \mu \mathrm{L}$ & $600 \mu \mathrm{L}$ \\
TransIT & $3 \mu \mathrm{L}$ & $9 \mu \mathrm{L}$ & $18 \mu \mathrm{L}$ \\
Plasmid DNA & $1 \mu \mathrm{g}$ & $3 \mu \mathrm{g}$ & $6 \mu \mathrm{g}$ \\
\hline \hline
\end{tabular}




\subsubsection{4. $\quad \underline{R N A \text { interference }}$}

To down regulate protein expression, siRNA was introduced into N2a cells. SiRNA was delivered to N2a cells by Oligofectamine (see Table 6) and cells were transfected $36 \mathrm{~h}$ later with the plasmids of interest, followed by medium exchange after $8 \mathrm{~h}$ and collection for extracellular vesicles. As a control, cells were mock transfected with oligofectamine reagent in the absence of siRNA.

\subsubsection{5. $\quad$ Collection of extracellular vesicles}

In general, after 8-16 $\mathrm{h}$ of transfection cells were washed three times with PBS and EVs derived from Oli-neu/N2a cells were collected at least for $16 \mathrm{~h}$ in serum free medium to eliminate any contaminations with serum derived exosomes.

\subsubsection{Luciferase activity assay}

HEK293 cells were cultivated as described in section 2.1.2.1 and transfected with a-Synuclein and SUMO-2-luciferase constructs (a-Syn fused to full length gaussia luciferase (syn phGluc); C-or N-terminal fragments of split phGluc fused to a-Syn (syn-S2) or SUMO-2 (SUMO-2-S3)). After $16 \mathrm{~h}$ of transfection the cells were washed with PBS and the general growth medium was replaced by medium without sera and phenol red to collect EVs for $48 \mathrm{~h}$. Thereafter, vesicles were prepared as described in section 2.2.3.1. Cells were washed with PBS and lysed in PBS using sonication. Luciferase activity from protein complementation was measured using same the amounts of total protein from both cell lysates and EV fractions in an automated plate reader at $480 \mathrm{~nm}$. Afterwards the cell permeable substrate coelenterazine (40 $\mu \mathrm{M}$;PJK $\mathrm{GmbH}$, Kleinbittersdorf, Germany) was added with a signal integration time of 2 seconds.

\subsubsection{Membrane preparation}

Cells were washed twice with ice cold PBS and collected into $200 \mu \mathrm{L}$ homogenization buffer (20 mM Na-HEPES, 1mM EDTA, $0.32 \mathrm{M}$ sucrose, $\mathrm{pH}$ 7.0). The cells were mechanically disrupted by 10 times pipetting up and down through a yellow pipette tip and finally $10 \mathrm{x}$ through a $27 \mathrm{G}$ needle. Cells were centrifuged at $4,000 \mathrm{rpm}$ for $5 \mathrm{~min}$ at $4{ }^{\circ} \mathrm{C}$. The postnuclear supernatant was then ultracentrifuged with $196,000 \times g$ for 30 min at $4{ }^{\circ} \mathrm{C}$, followed by a washing step with PBS. The pellet containing membrane fraction and cytosol were resolved in sample buffer and subjected to SDS-PAGE and Western blotting. 


\subsubsection{Extracellular vesicle purification and analysis}

\subsubsection{Ultracentrifugation}

Conditioned growth medium from cultured cells was collected as described in section 2.2.2.5. To purify EVs, an adapted protocol from (Strauss et al. 2010) was applied. Conditioned medium was collected and subjected to subsequent centrifugation steps performed at $4^{\circ} \mathrm{C}$, $3,500 \times g$ for $10 \mathrm{~min}, 2$ times at 4,500 $\times \mathrm{g}$ for $10 \mathrm{~min}, 10,000 \times \mathrm{g}$ for 30 minutes and $100,000 \times g$ with a TLA 100.3 rotor (Beckman-Coulter, $k$-factor 60.6) for $60 \mathrm{~min}$. Afterwards, the EV pellet was washed once with PBS (at 100,000 $\times g$ for $60 \mathrm{~min}$ ) before resuspended in protein loading buffer (see section 2.1.6.6). For the quantification of extracellular protein release, postnuclear supernatants of cell lysates that were gained by scraping the cells in $1 \%$ CHAPS lysis buffer (see section 2.1.6.5) and EV fractions were subjected to Western Blot analysis. The ratio of EV protein versus cellular protein levels was calculated by Image $\mathrm{J}$ analysis.

For the preparation of EVs from human cerebrospinal fluid $5 \mathrm{ml}$ cerebrospinal fluid was used for Western blot analysis. The samples were collected from consent informed patients The analysis of patient cerebrospinal fluid was approved by the ethical committee of the Medical Faculty, University Medicine Göttingen (IRB 02/05/09).

\subsubsection{Sucrose gradient ultracentrifugation}

For a cleaner purification, a 100,000 $\times \mathrm{g}$ pellet containing EVs were prepared as described above and resuspended in $400 \mu \mathrm{L}$ of $0.25 \mathrm{M}$ sucrose in $10 \mathrm{mM}$ HEPES, pH 7.4. Afterwards, the suspension was pulled 5 times through a $26 \mathrm{~g}$ needle to separate potential big clusters of EVs. The extracellular vesicle-sucrose suspension was then layered on top of a discontinuous sucrose density gradient consisting of 8 layers with $400 \mu \mathrm{L}$ each as listed in Table 10. The gradient was then centrifuged for $18 \mathrm{~h}$ and $200,000 \times \mathrm{g}$, at $4^{\circ} \mathrm{C}$ in a Sw $60 \mathrm{Ti}$ or a Sw $41 \mathrm{Ti}$ rotor (Beckman Coulter $\mathrm{GmbH}$, Krefeld, Germany) to separate vesicles according to their density (see Table 10). After centrifugation 8 fractions were recovered and diluted 1:6 with PBS. Thereafter, the diluted fractions were centrifuged for $1 \mathrm{~h}$ and $100.000 \mathrm{~g}$ at $4^{\circ} \mathrm{C}$ and the resulting pellet was resuspended in $15 \mu \mathrm{L}$ sample buffer and subjected to Western Blot analysis. 
Table 10: Sucrose density gradient

\begin{tabular}{|c|c|c|c|}
\hline $\begin{array}{l}\text { Molarity of } \\
\text { Sucrose [M] }\end{array}$ & $\begin{array}{l}\text { Corresponding } \\
\text { density }\left[\mathrm{g} / \mathrm{cm}^{3}\right]\end{array}$ & $\begin{array}{c}\text { Sucrose stock solution } \\
(2.5 \mathrm{M} \text { in } 20 \mathrm{mM} \text { HEPES) } \\
\text { for } 2.5 \mathrm{~mL}[\mathrm{~mL}]\end{array}$ & $\begin{array}{c}20 \mathrm{mM} \text { HEPES for } 2.5 \\
\mathrm{~mL}[\mathrm{~mL}]\end{array}$ \\
\hline $\begin{array}{l}0.25 \\
0.57 \\
0.89 \\
1.21 \\
1.53 \\
1.86 \\
2.18 \\
2,50\end{array}$ & $\begin{array}{l}1.03 \\
1.07 \\
1.11 \\
1.16 \\
1.20 \\
1.24 \\
1.27 \\
1.32\end{array}$ & $\begin{array}{c}0.25 \\
0.57 \\
0.89 \\
1.21 \\
1.53 \\
1.86 \\
2.18 \\
2.5\end{array}$ & $\begin{array}{c}2.25 \\
1.98 \\
1.61 \\
1.29 \\
0.97 \\
0.64 \\
0.32 \\
0\end{array}$ \\
\hline
\end{tabular}

\subsubsection{Nanoparticle tracking analysis (NTA)}

N2a cells were cultured as described in section 2.2.2.1 and transfected with TransIT®-LT1 (Mirus Bio LLC, Madison, WI, USA) according to the manufacturer's protocol. $8 \mathrm{~h}$ after transfection cells were washed with PBS and incubated in FCS-free DMEM for $16 \mathrm{~h}$. Then $200 \mu \mathrm{l}$ of culture medium was collected and centrifuged at $4^{\circ} \mathrm{C}, 5000 \mathrm{rpm}$ and for $10 \mathrm{~min}$ to remove cell debris. All samples were carried out at 1:1 dilution with PBS. For particle size determination and particle concentration, nanoparticle tracking analysis (NTA) was performed with a NanoSight LM10 instrument (NanoSight, Amesbury, United Kingdom). This experimental set up consists of a conventional optical microscope with a high resolution camera, which uses a $(<60 \mathrm{~mW}) 532 \mathrm{~nm}$ laser light to illuminate particles within a size of 50 $1000 \mathrm{~nm}$. The diluted samples were introduced into the sample chamber of the NanoSight LM10 analysis unit. While the particles in the laser beam undergo Brownian motion a video of these particle movements is recorded.

The NanoSight Tracking Analysis Software 2.3 then allows the automatic tracking of these particles and determines the particle concentration and the size distribution of the particles. Three videos with duration of 30 s and a camera level of 11 were recorded for each sample. For the analysis the detection threshold was set to 10 and at least 800 tracks were analysed for each video. The concentration of vesicles smaller than $120 \mathrm{~nm}$ was analysed, all bigger vesicles were excluded from the analysis. 


\subsubsection{Protein biochemistry}

\subsubsection{SDS-PAGE}

For protein separation according to their molecular weight, under denaturating conditions, sodium dodecyl sulfate polyacrylamide gel electrophoresis (SDS-PAGE) (Laemmli 1970) was performed by using the Bio-Rad Mini-PROTEAN® Tetra electrophoresis system (Bio-Rad Laboratories GmbH, Munich, Germany). The Bio-Rad Mini-PROTEAN® Tetra casting system was used to prepare two layered polyacrylamide gels. Composition of the upper stacking gel and the lower resolving gel are specified below in Table 11 and Table 12.

Table 11: Stacking gel (4\%)

\begin{tabular}{c|c|c|c|c}
\hline \hline Chemicals & $\mathbf{1 x}$ & $\mathbf{2 x}$ & $\mathbf{3 x}$ & $\mathbf{4 x}$ \\
\hline \hline $\mathrm{H}_{2} \mathrm{O}$ & $1.21 \mathrm{~mL}$ & $2.42 \mathrm{~mL}$ & $3.63 \mathrm{~mL}$ & $4.48 \mathrm{~mL}$ \\
Stacking buffer & $500 \mu \mathrm{L}$ & $1 \mathrm{~mL}$ & $1.5 \mathrm{~mL}$ & $2 \mathrm{~mL}$ \\
Acrylamide $(37.5: 1)$ & $540 \mu \mathrm{L}$ & $1.08 \mathrm{~mL}$ & $1.62 \mathrm{~mL}$ & $2.16 \mathrm{~mL}$ \\
$10 \%$ SDS & $20 \mu \mathrm{L}$ & $40 \mu \mathrm{L}$ & $60 \mu \mathrm{L}$ & $80 \mu \mathrm{L}$ \\
APS & $20 \mu \mathrm{L}$ & $40 \mu \mathrm{L}$ & $60 \mu \mathrm{L}$ & $80 \mu \mathrm{L}$ \\
TEMED & $3 \mu \mathrm{L}$ & $6 \mu \mathrm{L}$ & $9 \mu \mathrm{L}$ & $12 \mu \mathrm{L}$ \\
\hline \hline
\end{tabular}

Table 12: Resolving gel (12\%)

\begin{tabular}{c|c|c|c|c}
\hline \hline Chemicals & $\mathbf{1 x}$ & $\mathbf{2 x}$ & $\mathbf{3 x}$ & $\mathbf{4 x}$ \\
\hline \hline $\mathrm{H}_{2} \mathrm{O}$ & $1.66 \mathrm{~mL}$ & $3.32 \mathrm{~mL}$ & $4.98 \mathrm{~mL}$ & $6.64 \mathrm{~mL}$ \\
Resolving buffer & $1.3 \mathrm{~mL}$ & $2.6 \mathrm{~mL}$ & $3.9 \mathrm{~mL}$ & $5.2 \mathrm{~mL}$ \\
Acrylamide $(37.5: 1)$ & $2.04 \mathrm{~mL}$ & $4.08 \mathrm{~mL}$ & $6.12 \mathrm{~mL}$ & $8.16 \mathrm{~mL}$ \\
$10 \% \mathrm{SDS}$ & $50 \mu \mathrm{L}$ & $100 \mu \mathrm{L}$ & $150 \mu \mathrm{L}$ & $200 \mu \mathrm{L}$ \\
APS & $50 \mu \mathrm{L}$ & $100 \mu \mathrm{L}$ & $150 \mu \mathrm{L}$ & $200 \mu \mathrm{L}$ \\
TEMED & $2 \mu \mathrm{L}$ & $4 \mu \mathrm{L}$ & $6 \mu \mathrm{L}$ & $8 \mu \mathrm{L}$ \\
\hline \hline
\end{tabular}

For loading on the gel, samples (EVs and cell lysates) were mixed with denaturating protein loading buffer (see section 2.1.6.6) and incubated for $5 \mathrm{~min}$ at $95^{\circ} \mathrm{C}$. For the detection of PLP-myc protein, samples were incubated only at $55^{\circ} \mathrm{C}$ for $10 \mathrm{~min}$ to avoid assembly of multimers. After loading the sample were separated at $100 \mathrm{~V}$ for approximately $90 \mathrm{~min}$. To estimate the molecular weights of the analyzed proteins, the protein marker PageRuler $\circledast$ Plus Prestained Protein Ladder (Fermentas, St. Leon- Rot, Germany), was used for every run. 


\subsubsection{Western blotting}

After completion of gel electrophoresis, proteins were subjected to Western blot (Towbin et al. 1979). For the Western blot procedure a Mini-Trans Blot cell set up (Bio-Rad Laboratories $\mathrm{GmbH}$, Munich, Germany), according to the manufacturer's protocol, was used. By this procedure, proteins were transferred from a SDS-polyacrylamide gel onto a Whatman® Protran Nitrocellulose Transfer Membrane (Whatman GmbH, Dassel, Germany), at $100 \mathrm{~V}$ for $55 \min$ at $4^{\circ} \mathrm{C}$.

After the protein transfer a blocking step in $4 \%(\mathrm{w} / \mathrm{v})$ non-fat milk powder (AppliChem, Darmstadt, Germany) in 1\% PBS was applied, for $30 \mathrm{~min}$ at room temperature to avoid nonspecific binding of immunoglobulins. Thereafter, the membrane was incubated with primary antibodies in $0.05 \%$ PBST (Tween-20 in PBS) in dilutions according to Table 2, for $10-12 \mathrm{~h}$ at $4^{\circ} \mathrm{C}$. After washing three times for $15 \mathrm{~min}$ a specific secondary horse-radish peroxidase (HRP) coupled antibody was added to the membrane (1:2000 in PBST for EV fractions and 1:4500 in PBST for cell lysates) and incubated for $1 \mathrm{~h}$ at RT and washed 3 times for 25 min. Subsequently, detection of HRP coupled antibodies was achieved by an enhanced chemiluminescent reaction (Haan et al. 2007). Briefly, proteins were visualized by using ECL Western Blotting Substrate 1 and 2 (Thermo Fisher Scientific Inc.,Rockford, IL, USA) in equal volumes. Through, the enzymatic activity of the peroxidase, light was emitted. The signal of the light was then captured on X- Ray Films (CL-XPosure ${ }^{\text {TM }}$ Film, Thermo Fisher Scientific, Rockford,IL, USA) and the films were scanned and analyzed for light intensities by ImageJ (see Table 8).

\subsubsection{3. $\quad$ FCS/SIFT measurements}

For this assay the expression and purification of $\alpha$-Syn and sumoylated $\alpha$-Syn was performed as described previously (Krumova et al. 2011). The labelling of both proteins with Alexa Fluor-647-O-succinimidylester (Molecular Probes ${ }^{\circledR}$, USA) was carried out as described previously (Giese et al. 2005). Green labelled small unilamellar Dipalmitoyl-sn-glycero-3phospho-choline lipid vesicles (DPPC-SUV) were generated as described previously (Högen et al. 2012). Scanning for intensely fluorescent targets (SIFT) and Fluorescence correlation spectroscopy (FCS) measurements for the quantification of a-Syn vesicle binding were performed with an Insight Reader (Evotec-Technologies) with dual colour excitation at 488 and $633 \mathrm{~nm}$ as described before (Högen et al. 2012). All measurements were carried out after an incubation period of at least $30 \mathrm{~min}$ of DPPC-SUV with labelled $\alpha$-Syn. For equilibrium conditions, measurements were performed at least $2 \mathrm{~h}$ after addition of unlabelled non-sumoylated $\alpha$-Syn. 


\subsubsection{Electrochemiluminescence assay for quantification of $\alpha$-Synuclein}

For the quantification of $\alpha-S y n$ in cell lysates and EVs, derived from primary neurons, a slightly modified electrochemiluminescence assay was used (Kruse et al. 2012). Briefly, the antibody MJF-1, clone 12.1 (kindly provided by Dr. Liyu Wu, Epitopics Burlingame, USA), was coated on standard 96-well Multi-Array plates (Meso Scale Discovery, Gaithersburg, USA) and incubated over night at $4^{\circ} \mathrm{C}$. All additional steps were performed at room temperature. The plates were washed three times with $150 \mu \mathrm{L}$ PBS + $0.05 \%$ Tween-20. Subsequent blocking was performed with $150 \mu \mathrm{g}$ BSA (Meso Scale Discovery, Gaithersburg, USA) for $1 \mathrm{~h}$ with gently shaking at $300 \mathrm{rpm}$. A serial four-fold dilution of recombinant $\alpha$-Syn (kindly provided by Dr. Omar el- Agnaf, United Arab Emirates University, Al Ain, United Arab Emirates), starting at $25.000 \mathrm{pg} / \mathrm{ml}$, was used to prepare a standard curve. After washing as indicated above, $25 \mu \mathrm{L}$ of standards and samples were applied per well in duplicates. To secure a successful binding of the antibody to the samples, the plates were shaking for $1 \mathrm{~h}$ at $700 \mathrm{rpm}$ and then washed again as indicated above. Afterwards addition of $25 \mu \mathrm{L}$ of SulfoTAG labelled anti $\alpha-S y n$ clone 42 (BD Transduction Laboratories, Heidelberg, Germany) was added to achieve a final concentration of $1 \mu \mathrm{g} / \mathrm{mL}$ and incubated for $1 \mathrm{~h}$ at $700 \mathrm{rpm}$. Three washing steps followed, before $150 \mu \mathrm{L}$ of 2 x Read Buffer (Meso Scale Discovery, Gaithersburg, USA) was applied to each well and the plates were measured in a Sector Imager 6000 (Meso Scale Discovery, Gaithersburg, USA). The final data analysis was performed using MSD Discovery Workbench 3.0 Analysis Toolbox.

\subsubsection{Labelling of SUMO-2 with the ESPIT dye MFM}

SUMO-2 was labelled at its single cysteine (Cys) 52 with the ESPIT (excited state intramolecular proton transfer) probe MFM (Shvadchak et al. 2011). To uncover the Cys 52, SUMO-2 was pre-treated with $1 \mathrm{mM}$ DTT and a buffer exchange to $25 \mathrm{mM} \mathrm{PO}{ }_{4}-\mathrm{Na}, \mathrm{pH} 6.5$, without any sulfhydryl groups. Afterwards the protein concentration was measured and adjusted between $200 \mu \mathrm{M}$ and $350 \mu \mathrm{M}$, followed by the addition of the MFM dye (1-4 mg/mL) in 1-2 times excess and an incubation period for $12-24 \mathrm{~h}$ with gently mixing at $4^{\circ} \mathrm{C}$. Finally, 10 times excess of N-Methylmaleimide in DMSO was added and incubated for $30 \mathrm{~min}$ in the same conditions as before. This step is necessary to block any remaining free Cys groups. For purification the labelled protein was applied to a gravity PD 10 column (GE Healthcare Ltd., Little Chalfont, Buckinghmanshire, UK) and eluted with the same buffer, while collecting fractions of 5-10 drops. The fractions were checked for absorbance with a NanoDrop (PEQLAB Biotechnologie GmbH, Erlangen, Germany), pooled into one tube, aliquoted in small volumes and flash frozen in liquid $\mathrm{N}_{2}$. The labelled protein was stored at $-20^{\circ} \mathrm{C}$. 


\subsubsection{Lipid biochemistry}

\subsubsection{Preparation of Small Unilamellar Vesicles (SUVs)}

Small Unilamellar Vesicles (SUVs) were prepared by sonication as described previously (Huang et al. 1974), (Storch et al. 1986), (Falomir-Lockhart et al. 2011). Briefly, the composition of SUVs based on mixtures of POPC, POPC and PIPS (Avanti Polar Lipids, Inc., Alabaster, AL, USA). The relative molar compositions and approximate charge densities were as follows (POPC, 100; [0], POPC:POPS, 90:10; [-0.1], POPC:POPS:PI(3)P 85:10:5; [-0.13], POPC:POPS:PI(5)P 85:10:5; [-0.14); POPC:POPS:PI(3,5) $\mathrm{P}_{2}$ 85:10:5; [-0.2]; POPC:POPS:PI(4,5) $\mathrm{P}_{2}$ 85:10:5, [-0.2] and POPC:POPS:PI(3,4,5) $\mathrm{P}_{3}, 85: 10: 5$, [-0.25]).

At first the lipids were mixed from their chloroform stocks, in molar ratios indicated above, in clean glass balloons, followed by drying the mixture under a gently stream of nitrogen. Afterwards the dry lipid mixture was resuspended in a specific volume of buffer $(25 \mathrm{mM}$ HEPES, $100 \mathrm{mM} \mathrm{KCl}, \mathrm{pH}$ 7.26) and transferred to a falcon tube and sonicate in an ice water bath at least for $30 \mathrm{~min}$, until the solution appeared translucent.

After a $1 \mathrm{~h}$ centrifugation at $4^{\circ} \mathrm{C}$ and maximum speed, the vesicles were stored at least $5^{\circ} \mathrm{C}$ above the transition temperature of the lipid mixture and used within 10 days of their preparation. The vesicles were quantified by determining the inorganic phosphorus (Gomori 1942).

\subsubsection{Membrane binding assay of SUMO-2}

The measurements of labelled SUMO-2-MFM with SUVs were performed with a new 96 well microplate slope assay (to be published elsewhere). This assay offers several advantages compared to conventional fluorescence assays as lipids are added to proteins. Thereby, e.g. emission and scattering from lipids, photo-bleaching effects during the sequential addition of lipids and waste of material are avoided.

The strategy of "slopes" takes advantage of the maximal sensitivity of a titration performed with lipid concentrations in excess varied around the anticipated value of the dissociation constant $\mathrm{K}_{\mathrm{d}} \mathrm{S}$. The slopes measured for a small number of protein concentrations are plotted versus the lipid concentrations, from which $\mathrm{K}_{d}$ and the fluorescence enhancement factor are calculated from the relation: slope $=f O[1+(f e-1) \alpha$, where $f 0$ is the slope corresponding to 0 lipid concentration and $f e$ is the (enhanced) fluorescence of the bound protein relative to that of the free protein. Some major advantages of this assay are: the parallel readout in a microplate reader, the possible bottom readout with a small optical path length and therefore minimal scattering effects. Additionally, it is enough to use a minimal amount of reagents, endpoint determinations, that means no photo- bleaching effects. 
Solutions of SUMO-2-MFM (100 nM, $200 \mathrm{nM}$ and $300 \mathrm{nM})$ were prepared with 7 different SUV concentrations (0-120 $\mu \mathrm{M})$ in $25 \mathrm{mM}$ HEPES, $100 \mathrm{mM} \mathrm{KCl}, \mathrm{pH}$ 7.26. Afterwards $100 \mu \mathrm{l}$ of these 48 mixtures were added in duplicates to a 96 well quartz microplate (Hellma Analytics, Müllheim, Germany). After an incubation period of at least $10 \mathrm{~min}$ at room temperature, the fluorescence was recorded at $540 \mathrm{~nm}$ in BMG Pherastar plate reader (BMG Labtech, Ortenberg, Germany). The recording was applied with a bottom readout, well scan mode with a $10 \times 10$ matrix, a well scan diameter of $5 \mathrm{~mm}$ and with 25 flashes per well. Wells without lipid and/or protein were included to the data sets in order to establish blank values and the lipid contributions to the measured signal. Finally the data were analyzed with procedures implemented in Mathematica (Wolfram Research).

\subsubsection{NMR spectroscopy}

In order to study membrane binding of SUMO-2 NMR spectroscopy was performed. Thus $200 \mu \mathrm{M}$ of ${ }^{15} \mathrm{~N}$-labelled SUMO-2 in $20 \mathrm{mM} \mathrm{NaH} \mathrm{PO}_{4} / \mathrm{Na}_{2} \mathrm{HPO}_{4}, \mathrm{pH} 6.8,100 \mathrm{mM} \mathrm{KCl}, 1 \mathrm{mM}$ DTT was titrated with increasing concentrations $(8,16$ and $32 \mathrm{mM})$ of DHPC (1,2-dihexanoylsn-glycero-3-phosphocholine). ${ }^{1} \mathrm{H},{ }^{15} \mathrm{~N}$-HSQC spectra were acquired at $600 \mathrm{MHz}$ and $22 \stackrel{\circ}{\circ} \mathrm{C}$ on a triple resonance room temperature probe with 16 transients, $2084 \times 256$ total points and widths of $8418 \times 2129 \mathrm{~Hz}\left({ }^{1} \mathrm{H} \times{ }^{15} \mathrm{~N}\right)$. Carrier frequencies were set to the water resonance for ${ }^{1} \mathrm{H}$ and to $117 \mathrm{ppm}$ for ${ }^{15} \mathrm{~N}$. Resonance assignments were taken from BMRB entry 11267. The normalized weighted average chemical shift difference for the amide proton and nitrogen were calculated according to $\Delta \delta(H N)=\left[\Delta \delta_{H}{ }^{2}+\left(0.2^{*} \Delta \delta_{N}\right)^{2}\right]^{1 / 2}$.

\subsubsection{Immunocytochemistry}

\subsubsection{Immunofluorescence staining}

Proteins were labeled with specific primary antibodies and fluorophore-labeled secondary antibodies to determine their localization in cultured N2a cells. All steps of the staining protocol were carried out at RT. N2a cells were grown on PLL-coated glass coverslips, washed once with PBS and fixed then with PFA (Paraformaldehyde) (4\% PFA in PBS, $\mathrm{pH} 7.4)$ for $25 \mathrm{~min}$. Thereafter coverslips were washed three times with PBS and cells were permeabilized in $0.1 \%(\mathrm{v} / \mathrm{v})$ Triton X-100 (in PBS), that allows the antibodies to enter the cell. Subsequently the cells were washed immediately three times and covered with $100 \%$ blocking solution (see below) for 35 min to avoid unspecific binding of the antibodies. 
Primary antibodies (see Table 2) were diluted in $10 \%$ blocking solution and incubated with the cells in a dark and humidity chamber for $1 \mathrm{~h}$ at RT. After three washing steps with $1 \mathrm{x}$ PBS for 5 min, cells were incubated with fluorophor-conjugated secondary antibodies in $10 \%$ blocking solution for $1 \mathrm{~h}$, again in a dark and humidity chamber. Thereafter, the cells were washed 3 times with $1 \times$ PBS for 5 min and once with bi- distilled $\mathrm{H}_{2} \mathrm{O}$ to remove remaining salt traces, followed by mounting the glass coverslips onto glass slides with a drop of mowiol (see below) and dried overnight. For long term period the slides were kept in the dark and stored at $4^{\circ} \mathrm{C}$.

$100 \%$ Blocking solution

$2 \%$ BSA

$2 \% \quad$ FCS

$0.2 \%$ Gelatin, from cold water fish skin

add $10 \mathrm{~mL} 10 \times$ PBS

Fill up to $100 \mathrm{~mL}$ with bi- distilled $\mathrm{H}_{2} \mathrm{O}$. The solution was aliquoted to $5 \mathrm{~mL}$ and stored at $-20^{\circ} \mathrm{C}$.

\section{Preparation of $16 \%$ paraformaldehyde (PFA)}

For the preparation of $16 \%$ paraformaldehyse (PFA) solution, $16 \mathrm{~g}$ PFA (AppliChem GmbH, Darstadt, Germany) was mixed with $70 \mathrm{~mL}$ bi-distilled $\mathrm{H}_{2} \mathrm{O}$ and dissolved by heating to $60^{\circ} \mathrm{C}$. Thereafter 2-3 pellets $\mathrm{NaOH}$ were added, resulting in a noticeable cooling of the solution, followed by the addition of $10 \mathrm{~mL} 10 \times$ PBS and the chilling to room temperature. Finally the $\mathrm{pH}$ was adjusted to 7.4 and the solution was filled up to $100 \mathrm{~mL}$ with bi-distilled $\mathrm{H}_{2} \mathrm{O}$. The solution was separated to $3 \mathrm{~mL}$ aliquots and stored at $-20^{\circ} \mathrm{C}$.

\section{Preparation of mowiol solution}

To prepare the mounting solution, $2.4 \mathrm{~g}$ mowiol (GmbH, Darstadt, Germany) and $6 \mathrm{~g}$ glycerol were mixed and incubated at room temperature for $2 \mathrm{~h}$ with gentle agitation. Thereafter, $12 \mathrm{~mL} 0.2 \mathrm{M} \mathrm{Tris} / \mathrm{HCl}(\mathrm{pH}$ 8.5) were added and the solution was mixed under heating to $50^{\circ} \mathrm{C}$. A subsequent centrifugation step at $5,000 \times \mathrm{g}$ secure the clearance of the solution, followed by the addition of the anti-fading reagent 1,4-Diazabicyclo[2.2.2] octan (DABCO) in a final concentration of $24 \mathrm{mg} / \mathrm{ml}$ (Sigma-Aldrich St. Louis, MO, USA). Finally the mowiol solution was aliquoted and stored at $-20^{\circ} \mathrm{C}$. 


\subsubsection{Microscopy}

\subsubsection{Confocal microscopy}

To visualize and record the localization of proteins, which were stained with fluorescent antibodies, in PFA fixed cells, confocal microscopy was applied. The images were acquired with a Leica DMIRE2 microscope with a $63 \times$ oil-immersion objective and a Leica TCS SP2 AOBS confocal laser scanning setup (Leica Microsystems, Darmstadt, Germany).

\subsubsection{Electron microscopy}

EVs were prepared from cerebrospinal fluid and culture medium as described in section 2.2.3.1. The $100,000 \times \mathrm{g}$ pellet was fixed with $4 \%$ PFA and was adsorbed to glow-discharged Formvar-carbon-coated copper grids by floating the grid for $10 \mathrm{~min}$ on $5 \mu \mathrm{l}$ droplets on Parafilm. The grids were negatively stained with $2 \%$ uranyl acetate containing $0.7 \mathrm{M}$ oxalate, pH7.0, and imaged with a LEO EM912 Omega electron microscope (Carl Zeiss, Jena, Germany). Digital micrographs were obtained with an on-axis $2048 \times 2084$ CCD camera (Proscan GmbH, Scheuring, Germany). (Electron microscopic imaging of EVs was kindly performed by Dr. Wiebke Möbius, MPI for experimental medicine, Göttingen).

\subsubsection{Image processing and statistical analysis}

\subsubsection{Quantification of extracellular vesicle secretion}

To compare the relative EV release, EV pellets and the corresponding cell lysates were subjected to Western blotting as described in section 2.2.4.1 and 2.2.4.2. After developing of the Western blot membranes on X-ray films (CL-XPosure ${ }^{\mathrm{TM}}$ Film, Thermo Fisher Scientific, Rockford,IL, USA), the films were scanned and analysed with ImageJ software for the signal intension of protein bands on the X-ray films. As a degree of $\mathrm{EV}$ release, the ratio of signal intensities of EVs versus corresponding cell lysates was calculated from at least 4-13 independent experiments. 


\subsubsection{2. $\quad$ Statistical analysis}

Data were statistical analysed with MS Office Excel 2007 (Microsoft Deutschland GmbH, Berlin, Germany). For descriptive statistics, mean and standard error of the mean (SEM) of a data set were calculated and illustrated with MS Office Excel 2007. For the comparison of two independent groups with normal distribution of sample sets and equal variance, the parametric Student's t-test was used. A data group which displays a p-value less than 0.05 was regarded as significantly different. 


\section{Results}

Most of these results have been published in:

\section{Extracellular vesicle sorting of $\alpha$-Synuclein is regulated by sumoylation}

Marcel Kunadt, Katrin Eckermann, Anne Stuendl, Jing Gong, Belisa Russo Katrin Strauss, Surya Rai, Sebastian Kügler, Lisandro Falomir Lockhart, Martin Schwalbe, Petranka Krumova, Luis M. A. Oliveira, Mathias Bähr, Wiebke Möbius, Johannes Levin, Armin Giese, Niels Kruse, Brit Mollenhauer, Ruth Geiss-Friedlander, Albert C. Ludolph, Axel Freischmidt, Marisa S. Feiler, Karin M. Danzer, Markus Zweckstetter, Thomas M. Jovin, Mikael Simons, Jochen H. Weishaupt, Anja Schneider

Acta Neuropathol DOI 10.1007/s00401-015-1408-1

The results displayed in Fig. 9 A, Fig. 10 A, Fig. 11, Fig. 13, Fig. 17 and Fig. 18 were first performed by Surya Rai, a former master student under the supervision of Prof. Dr. Anja Schneider. In the course of this thesis, the experiments were repeated to increase the number of performed experiments and to improve the significance.

\section{1. $\alpha$-Synuclein is released in extracellular vesicles}

In neurodegenerative diseases extracellular vesicles (EVs) have been proposed to be potential carriers of misfolded proteins and thereby may be responsible for the spreading of the disease pathology (Aguzzi et al. 2009). In this study we aimed to investigate how $\alpha$-Syn is sorted into EVs.

\subsection{1. $\quad \alpha$-Synuclein is released in extracellular vesicles derived from N2a cells}

For the preparation of EVs, the conditioned medium was collected and subjected to subsequent centrifugation steps (see section 2.2.2.5 and section 2.2.3.1). In a final ultracentrifugation step at $100.000 \times g$ for $1 \mathrm{~h}, \mathrm{EVs}$ were pelleted as previously described (Trajkovic et al. 2008). We further refer to this $100.000 \times g$ pellet as EV pellet (P100). The P100 and the cell lysate of the corresponding secreting parental cells were subjected to Western blot analysis and probed with an antibody against $\alpha$-Synuclein. As shown in Fig. $6 \mathrm{~A}$ a-Syn was enriched in the P 100. 
As a positive control the EV fraction and the corresponding lysates were also stained with the EV marker proteins Alix (AIP-1) and Flotilin 2 (Flot-2). In addition to the signal for a-Syn we also found intense signals for both EV marker proteins, Alix and Flot-2 in the P100. A contamination of the P100 with cellular compartments, membrane particles or other vesicles than EVs could be excluded by the absence of a signal for cellular compartments, like the ER marker protein Calnexin (Fig. 6 A).

A

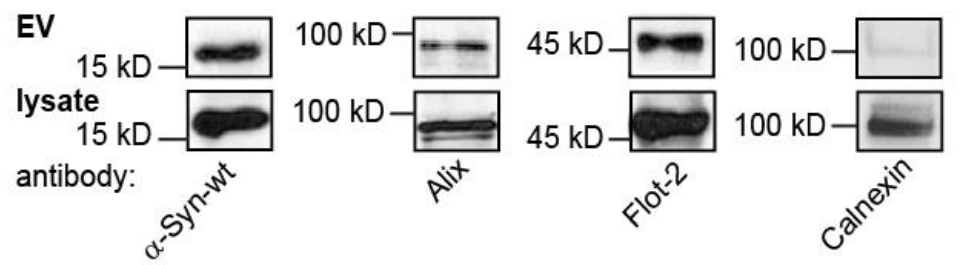

C

B
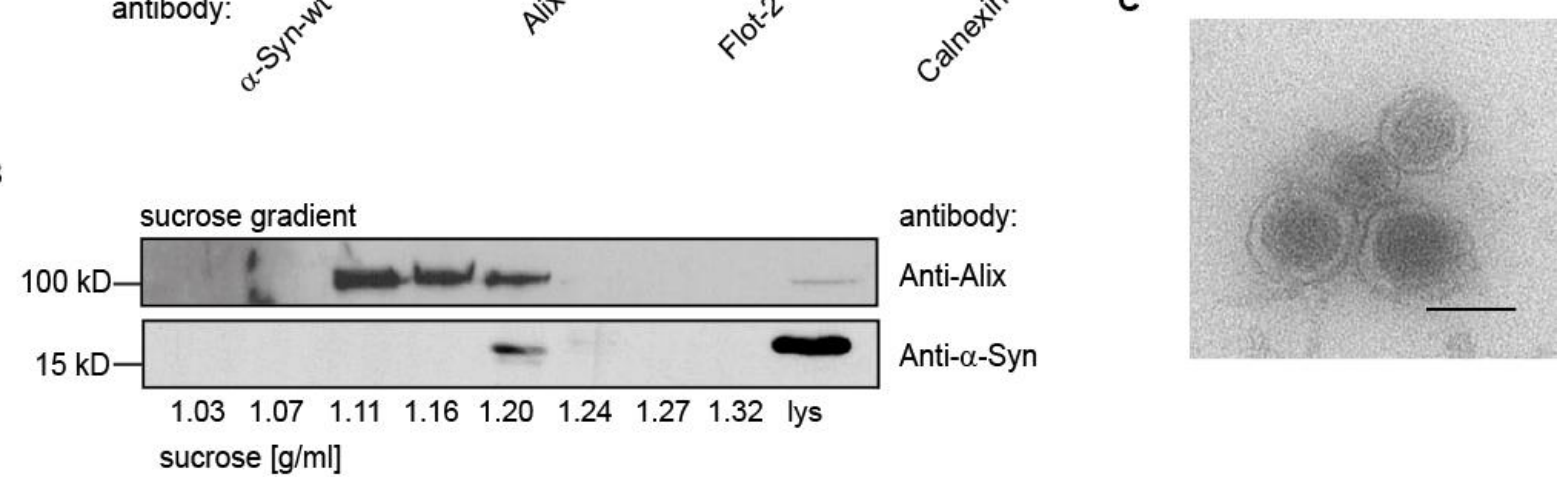

Fig. 6: $\alpha$ - Synuclein is released in extracellular vesicles derived from N2a cells

(A) Cultured medium of $\mathrm{N} 2 \mathrm{a}$ cells was collected and subjected to subsequent centrifugations steps to clear the medium from cell debris, dead cells and macrovesicles with $1 \times 10$ min at $3500 \times g, 2 \times 10$ min at $4500 \times g$ and $1 \times$ $30 \mathrm{~min}$ at $10,000 \times \mathrm{g}$. In a final centrifugation step the EVs were pelleted. The whole EV pellet and $10 \mu \mathrm{l}$ of the corresponding cell lysates were subjected to Western Blot analysis. The P100 pellet is immune positive for $\alpha$-Syn and the EV marker proteins Flot-2 and Alix, but negative for the ER marker Calnexin. (B) For a broader purification the P100 was loaded on top of a sucrose gradient (1.03-1.32 g/mL) and ultracentrifuged for $16 \mathrm{~h}$ at $200,000 \times g$. The collected fractions were ultracentrifuged again and the pellets as well as the corresponding lysates were subjected to Western Blot analysis and immune stained against $\alpha$-Syn and Alix. The detected signals corresponded to known densities for EVs ranging from 1.11 to $1.20 \mathrm{~g} / \mathrm{mL}$. (C) EVs derived from N2a cells were processed to electron microscopy and showed their typical cup shaped morphology (scale bar $100 \mathrm{~nm}$ ).

In another experiment we subjected the P100 to sucrose density ultracentrifugation, to get a higher purity level of the EV fraction as well as to further characterise the previous P100. The gradient was centrifuged at $200,000 \times g$ for $16 \mathrm{~h}$. After the ultracentrifugation step 8 fractions, corresponding to densities between $1.03-1.32 \mathrm{~g} / \mathrm{mL}(0.25-2.5 \mathrm{M})$, were collected and diluted $1: 6$ with PBS. These fractions were processed to Western Blot analysis and immunostained for a-Syn and for the EV marker protein Alix. As shown in Fig. 6 B signals were detected for $\alpha-S y n$ in the fraction of $1.20 \mathrm{~g} / \mathrm{mL}$ and for Alix in fractions of $1.11,1.16$ and $1.20 \mathrm{~g} / \mathrm{mL}$. 
This is in line with the previous described flotation behaviour of EVs (Fauré et al. 2006, Théry et al. 2006). To visualize EVs we subjected the $100.000 \times g$ pellet to electron microscopy and negatively stained the pellets with $1 \%$ uranyl acetate. We found the typical cup shaped morphology (Simons et al. 2009) with diameter between $50 \mathrm{~nm}$ and $100 \mathrm{~nm}$, as previously observed by transmission and cryo-electron microscopy (Conde-Vancells et al. 2008) (Fig. $6 \mathrm{C})$. Taken together, these data demonstrate that $\alpha-S y n$ is released within EVs derived from $\mathrm{N} 2 \mathrm{a}$ cells and that we are able to recover material with our EV purification protocol.

\subsection{2. $\quad \alpha$-Synuclein is localized in extracellular vesicles in vivo}

It is not known whether a-Syn is present in EVs in vivo. To address this issue we firstly analysed whether $\alpha-S y n$ is present in EVs in the human central nervous system (CNS). Therefore, we prepared EVs from cerebrospinal fluid (CSF) after the written informed consent was given of patients with PD. Analysis of patient CSF was approved by the ethical committee of the Medical Faculty, University Medicine Goettingen (IRB 02/05/09). The CSF was subjected to a series of centrifugation steps to clear the CSF from cell debris with $1 \mathrm{x}$ at $3500 \times g$ for $10 \mathrm{~min}$ (P3), $2 \times$ at $4500 \times g$ for $10 \mathrm{~min}$ (P4), $1 \times$ at $10.000 \times g$ for $30 \mathrm{~min}$ (P10) and a final $100.000 \times g$ ultracentrifugation step (P100). Pellets of each centrifugation step and the EV pellet (P100) were subjected to Western blot analysis and probed with Flot-2 and Calnexin antibodies. As shown in Fig. 7 A Flotillin 2 was enriched in the EV fraction and a contamination of the $100,000 \times g$ pellet could be excluded by immunostaining for the ER marker Calnexin. 
A

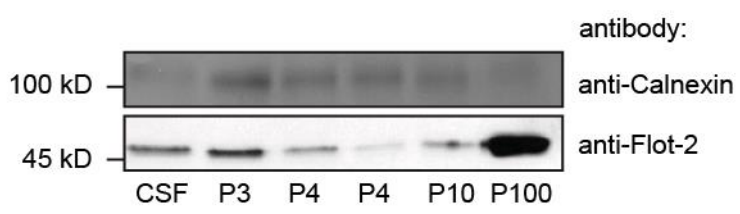

C

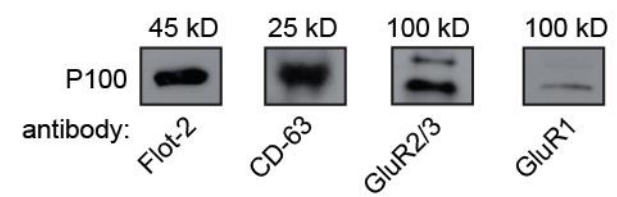

E

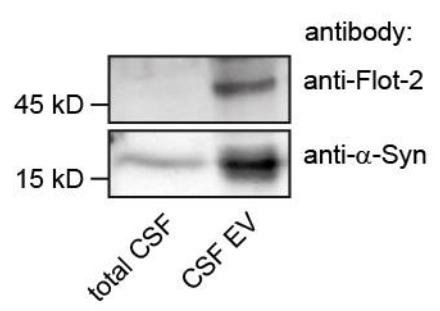

B

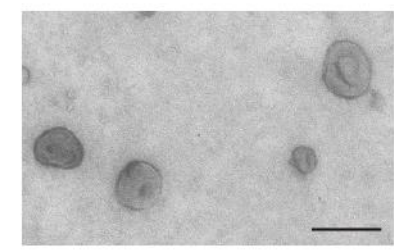

D

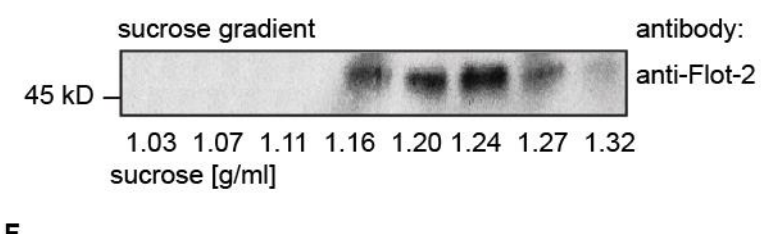

$\mathbf{F}$

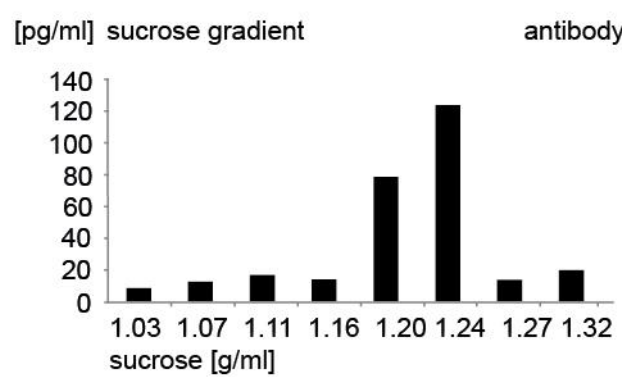

Fig. 7: Characterization of extracellular vesicles in cerebrospinal fluid

(A) Cerebrospinal fluid was processed to a series of centrifugation steps and each fraction as well as the P 100 was immunostained in Western blot. (B) Part of the P 100 was negatively stained with $1 \%$ uranyl acetate and the EVs were visualized by electron microscopy (scale bar $100 \mathrm{~nm}$ ). (C) Immunostaining of $100.000 \times \mathrm{g}$ pellets against various microsomal and EV marker proteins. (D) Discontinuous sucrose density gradient $(0.25 \mathrm{M}-2.5 \mathrm{M})$ was analysed by Western Blot for the presence of Flot-2. (E) EVs were prepared from $5 \mathrm{~mL} C S F$ and $20 \mu \mathrm{L}$ of total CSF and the corresponding $100.000 \times g$ pellet were subjected to Western blot analysis. One representative blot out of 3 different patient samples is shown. (F) A 100,000 x $g$ pellet of a Parkinson dementia CSF sample was loaded on a discontinuous sucrose gradient $(0.25 \mathrm{M}-2.5 \mathrm{M})$ and $\alpha$-synuclein was quantified in each fraction via an electrochemiluminescence assay.

Electron microscopy of the resulting P100 revealed 50-100 nm structures with the typical cup shaped morphology for EVs (Fig. 7 B). The $100.000 \times$ g pellet was also enriched for EV marker protein CD63 as well as for the Glutamate Receptors- 1, -2 and -3. This latter indicates that CSF EVs are at least partially derived from the central nervous system. Microsomal proteins such as the ER marker Calnexin and the trans golgi network (TGN) protein $\gamma$-Adaptin were absent (data not shown), thus excluding microsomal contamination of the EV preparation (Fig. $7 \mathrm{C}$ ). On a sucrose gradient Flotillin-2 positive EVs showed a consistent floating behaviour as supported by previously published results (Baietti et al. 2012). Flotillin-2 was enriched at a density of 1.16-1.24 g/mL (Fig. 7 D). 
To elucidate whether $\alpha$-Syn is enriched in the P100 of CSF in comparison to total cerebrospinal fluid, we processed total CSF and the corresponding $100.000 \times g$ pellet to Western blot analysis and the samples were immunostained for $\alpha$-Syn. As shown in Fig. $7 \mathrm{E}$, the $100,000 \times g$ pellet revealed an enriched $\alpha$-Syn signal compared to total CSF. In addition we performed a sucrose density ultracentrifugation experiment with a $100,000 \times g$ pellet of a Parkison's disease CSF sample. In this experiment EVs of CSF samples were isolated and the resulting $100.000 \times g$ pellet was subjected to a discontinuous sucrose gradient, consisting of 8 different layers (0.25 M-2.5 M, see section 2.1.6.3). Subsequent detection of a-Syn by an electrochemiluminescence assay (see section 2.2.4.4) revealed flotation behaviour of CSF derived $\alpha$-Syn, similar to the EV marker protein Flotilin-2 (Fig. 7 F). Taken together, all these findings indicate, that $\alpha$-Syn associated EVs are present in the CNS in vivo

\subsection{3. $\alpha$-Synuclein is predominantly localized in the lumen of EVs}

We next wanted to clarify whether $\alpha$-Syn is either localized in the lumen of EVs or rather attached to the outer membrane. To this end, we transiently transfected N2a cells with a wild-type a-Syn plasmid and EVs were prepared from cultured medium and processed to subsequent centrifugations steps, as described previously in section 2.2.3.1. The P100 was resuspended in PBS and divided into two equal parts. One part was digested with trypsin and the other only with PBS as a control. The silver gel shows degradation bands for the trypsin treated P100 pellet compared to the non-trypsinized control (PBS treated) (Fig. 8 A). Western Blot analysis showed that the content of the bona fide intraluminal protein Flotilin-2 and $\alpha$-Syn was unaltered by trypsin treatment, which indicates that $\alpha$-Syn is localised in the lumen of EVs (Fig. 8 B). 
A

B

\section{Silver staining}

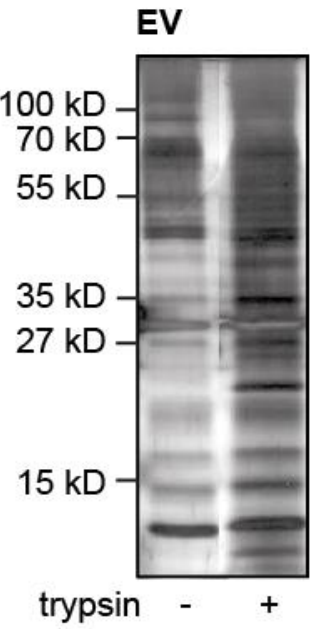

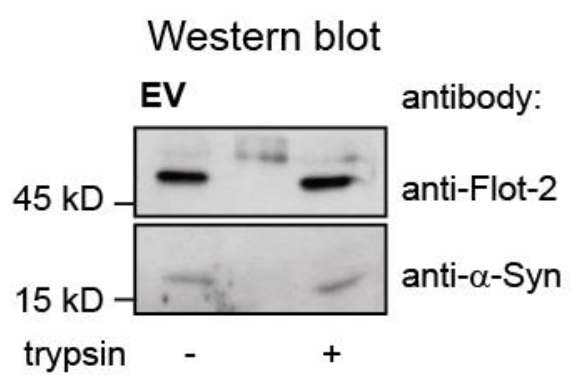

D

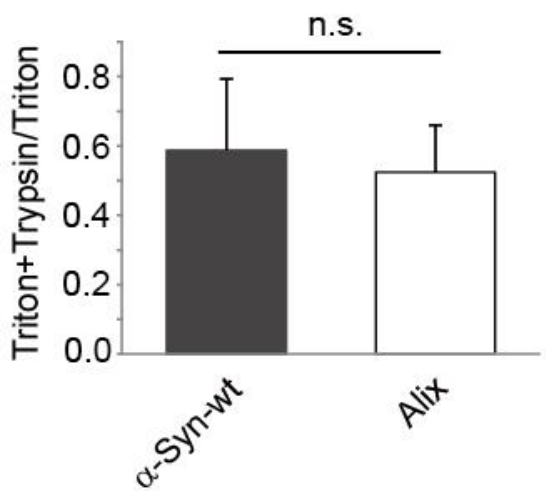

Fig. 8: $\alpha$-Syn is localised in the intraluminal compartment of extracellular vesicles

(A) The $100.000 \times g$ pellets were digested in $0.0125 \%$ and incubated for $5 \mathrm{~min}$ at $37^{\circ} \mathrm{C}$ (right lane). As a control the other half of the pellet was incubated in PBS under the same conditions as used for the trypsin treatment. Trypsination reaction was stopped by adding sample buffer. The efficiency of the reaction was controlled by SDSPAGE and silver staining of the gel. (B) Western Blot analysis of not- trypsinized (left) and trypsinized (right) EVs. The membrane was immunostained against Flotilin-2 and a-Syn. (C) EV Pellets were resuspended in PBS and $0.00084 \%$ Trypsin and incubated either in the presence (right lane) or in the absence (left lane) of $1 \%$ Triton $\mathrm{X}-100$ for $3 \mathrm{~min}$ at $37^{\circ} \mathrm{C}$. Samples were analyzed by Western Blot for staining against Alix and $\alpha$-Syn. (D) The level of degradation was quantified by calculating the ratio under trypsin plus triton condition versus trypsin without triton condition. Values are given as mean + SEM, n.s. $=$ not significant.

To further investigate whether $\alpha$-Syn is attached to the outer membrane or resides within the lumen of EVs, we performed the same experiment as described above, but incubated the pellet either in the absence or presence of $1 \%$ Triton (to disrupt the membrane of EVs). 
The reaction was stopped by adding sample buffer (see section 2.1.6.6) and the probes were subjected to Western blot analysis and stained again Alix (AIP1) which also resides in the lumen of EVs and for a-Syn.

In contrast to the treatment without $1 \%$ Triton, $\alpha$-Syn was degraded to a similar extent as Alix when the EV pellet was trypsinized in the presence of $1 \%$ Triton (Fig. $8 \mathrm{C}+\mathrm{D}$ ).

Taken together, the trypsin digestion in the absence and in the presence of $1 \%$ Triton revealed that a-Syn resides within the EVs rather than being attached to the outer membrane. 


\subsection{The extracellular release of $\alpha-S y n u c l e i n$ is regulated by membrane binding}

To answer the question how $\alpha$-Syn is targeted into the lumen of EVs, we hypothesised that cytosolic proteins such $\alpha$-Syn need to bind to the limiting membrane of late endosomes. Therefore, we hypothesized that membrane binding should have an impact on the EV release of $\alpha$-Syn. Membrane binding of $\alpha$-Syn involves the binding of amino acids 3-25 (Bartels et al. 2010, Bodner et al. 2010).

Therefore, we transiently transfected $\mathrm{N} 2 \mathrm{a}$ cells with an $\mathrm{N}$-terminal deletion construct of a-Syn, lacking the amino acids 2-19 (Karube et al. 2008, Bartels et al. 2010). To determine the membrane binding affinity of a $\Delta \mathrm{N}$-truncated version of $\alpha$-Syn, we scraped the cells in a homogenisation buffer (see section 2.1.6.4) and mechanically disrupt them by passing through a $27 \mathrm{G}$ needle. In order to remove cell and nuclei debris we processed the suspension to a subsequent centrifugation step at $4000 \mathrm{rpm}$ and $4^{\circ} \mathrm{C}$ for $5 \mathrm{~min}$. A final ultracentrifugation step at $196,000 \times g$ for $30 \mathrm{~min}$ at $4^{\circ} \mathrm{C}$ was necessary to separate cytosolic and membrane fractions. Finally, both fractions were subjected to Western blot analysis. The membrane pellets and the corresponding cytosolic fractions were immunostained with an antibody against $\alpha-S y n$.

As present in Fig. $9 \mathrm{~A}$, the membrane binding propensity of the $\mathrm{N}$-terminally deletion construct of a-Syn was significantly decreased, compared to an $\alpha$-Syn wt construct in transiently transfected N2a cells. To control the separation of cytosolic and membrane fractions, the blot membranes were probed with an antibody against Glycerinaldehyd-3phosphat-dehydrogenase (GAPDH) as a cytosolic marker or against $\beta 5$-Integrin as a membrane marker (Fig. $9 \mathrm{~B}$ and C). 
A

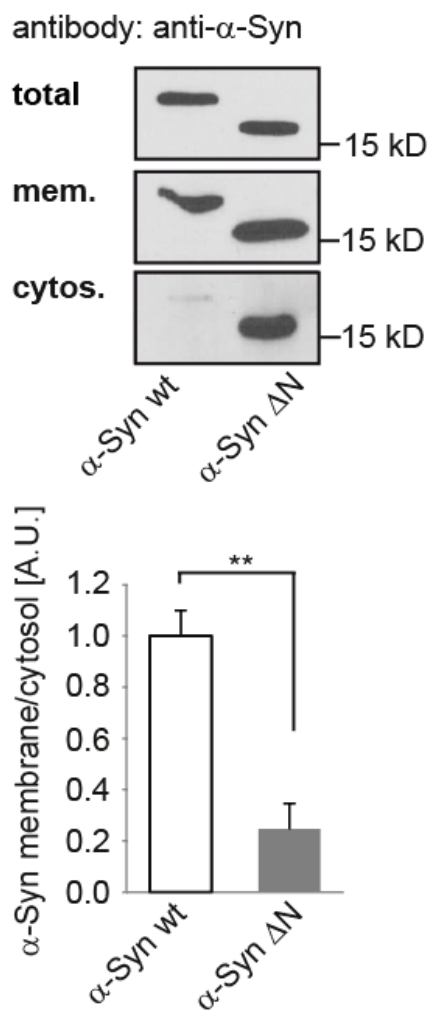

B

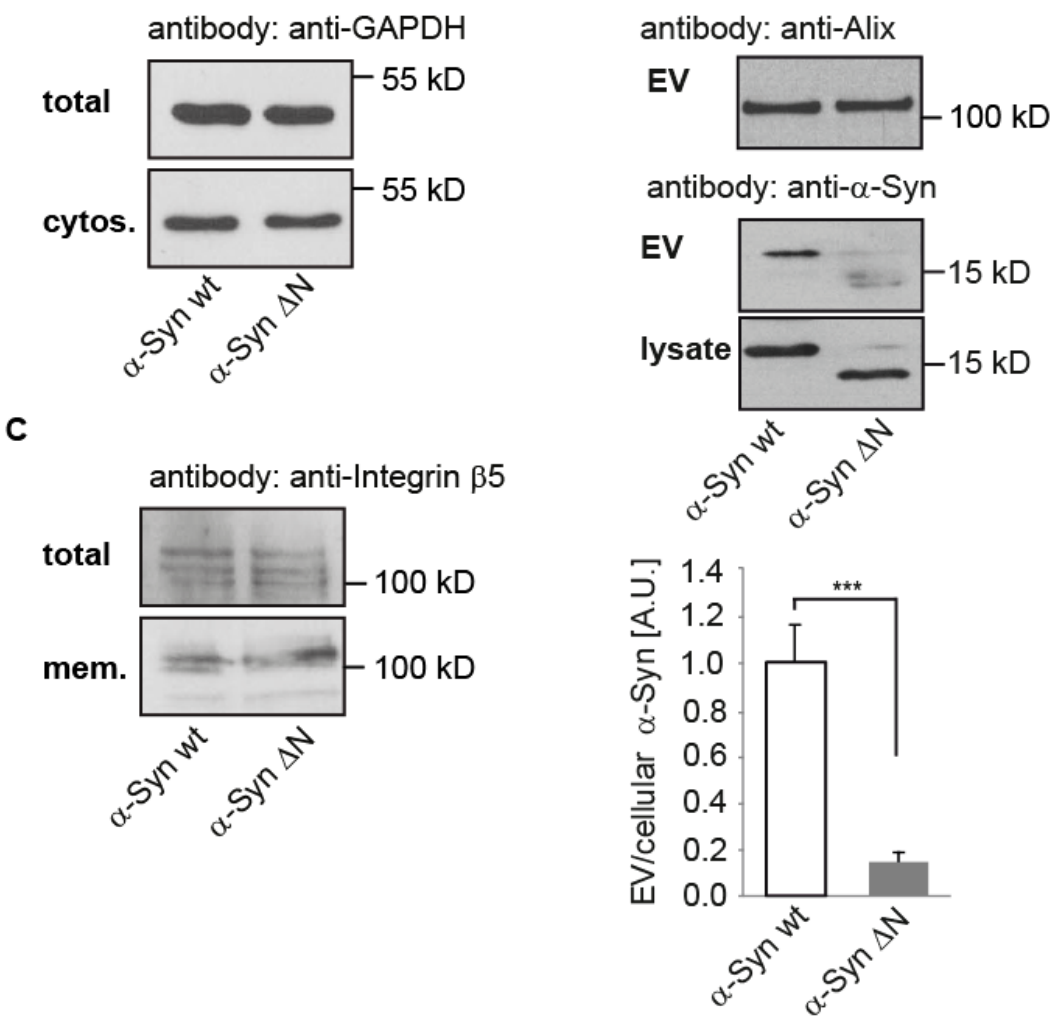

Fig. 9: Membrane binding regulates release of $\alpha$-Synuclein within extracellular vesicles

(A) Plasmids encoding for $\alpha$-Syn wt and the N-terminally truncated version $(\Delta N)$ of $\alpha$-Syn were transiently transfected in N2a cells. The cells were scraped, mechanically disrupted and subjected to different centrifugation steps to separate membrane and cytosolic fractions. Complete membrane pellet and a proportion of the cytosolic supernatant were processed to Western blot analysis (top). To quantify the ratio of membrane associated $\alpha-$ Synuclein, the ratio of protein intensity in the membrane fraction versus protein intensity of the cytosolic fraction was determined (bottom). Values are given as mean + SEM from $n=8$ experiments; ${ }^{* *}$ indicates $p<0.005$. $(\mathbf{B}+\mathbf{C})$ The blots in (A) were re-probed with an antibody against GAPDH as a cytosolic marker and against $\beta 5$-Integrin as a membrane marker, respectively. (D) N2a cells were transfected with the same constructs as in (A). EVs were prepared from cultured medium of N2a cells and the ratio of EVs to the corresponding cell lysate protein was quantified upon Western blot analysis (top) and signal intensity was measured with ImageJ (bottom). As a positive control for EVs, the membrane was re-probed with an antibody against the extracellular marker protein Alix. $(\mathrm{A}+\mathrm{D})$ Results are given as mean + SEM from $n=8$ independent experiments; ${ }^{* * *}$ indicates $p \leq 0.001$; student's 2-side t-test.

Next, we wanted to know whether membrane binding indeed regulates the release of $\alpha$-Syn within EVs. Therefore we transfected N2a cells with the N-terminal deletion construct of $\alpha$-Syn. To quantify the EV release of $\alpha$-Syn we collected and prepared vesicles as described in sections 2.2.2.5 and 2.2.3.1. To determine the EV release of both constructs, we subjected the EV pellet and the corresponding parental cell lysates to Western blot analysis and probed the membrane with an antibody against a-Syn. 
As shown in Fig. $9 \mathrm{D}$ (upper panel), the $\mathrm{N}$-terminal deletion construct of $\alpha$-Syn was largely excluded from the EV fraction in comparison to the $\alpha$-Syn wt construct. Altogehther, this data demonstrates that membrane binding is required for the sorting of a-Syn into EVs.

\subsection{SUMOylation regulates membrane binding and extracellular vesicle release of $\alpha-S y n u c l e i n$}

We hypothesized that SUMOylation might regulate the release of $\alpha$-Syn by modulating the binding of a-Syn to lipid membranes. In a previous study the two major SUMOylation sites in a-Syn (K96 and K102) were described to be in close proximity to the membrane interacting $\alpha$-helical regions of a-Syn (Krumova et al. 2011). We wondered whether SUMOylation of these sites might modulate its interaction of with lipid membranes.

\subsubsection{SUMOylation modulates membrane binding of $\alpha$-Synuclein}

We transiently transfected $\mathrm{N} 2 \mathrm{a}$ cells with myc- $\alpha$-Syn constructs either bearing the K96R $\mathrm{K} 102 \mathrm{R}$ double mutation at both sumoylation sites which account for more than $50 \%$ of protein's SUMO modification, further referred to as $2 \mathrm{KR}$ mutant, or the D98A E104A double mutation, further referred to as 2 AA mutant, which disrupts the consensus sequence for sumoylation (Krumova et al. 2011).

After transfection, N2a cells were mechanically disrupted followed by a subsequent centrifugation step to remove cell and nuclei debris. The postnuclear supernatant was then processed to an ultracentrifugation step to separate membrane pellet and cytosolic supernatant. Thereafter, SDS-PAGE and subsequent Western Blot analysis was performed, to investigate the membrane binding of both SUMO-deficient mutants.

As displayed in Fig. $10 \mathrm{~A}$ we found that the membrane binding of both SUMO-deficient mutants ( $\alpha$-Syn $2 K R$ and $\alpha$-Syn 2AA) was significantly attenuated when compared to a myc$\alpha$-Syn wt construct. To verify if the separation of cytosolic and membrane fractions was successful, we re-probed the membrane with an antibody against GAPDH, as a positive control for the cytosolic fraction and with an antibody against $\beta 5$-Integrin as well, as a positive control for the membrane fraction (Fig. $10 \mathrm{~B}$ and $\mathrm{C}$ ). 
A

antibody: anti- $\alpha-S y n$

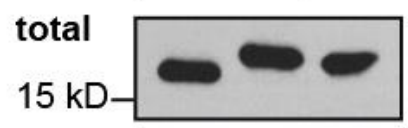

mem.

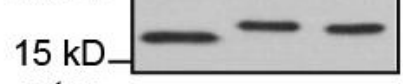

cytos.

$15 \mathrm{k}$
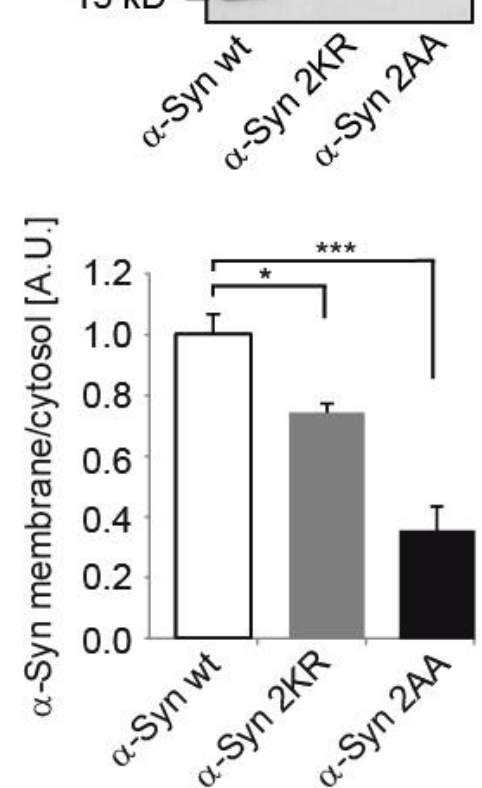

B

antibody: anti-GAPDH

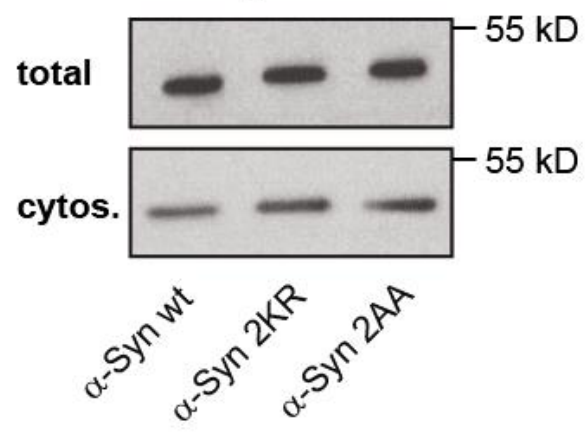

C

antibody: anti-Integrin $\beta 5$

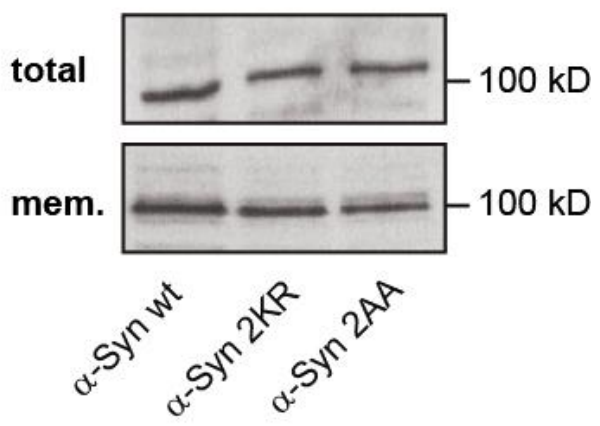

Fig. 10: Membrane binding of SUMO- deficient $\alpha$-Syn mutants 2 KR and 2 AA

(A) N2a cells were transiently transfected with the indicated $\alpha$-Syn mutant constructs and a wt construct of $\alpha$-Syn. Cells were scraped and resuspended in homogenisation buffer and mechanically disrupted by passing through a 27G needle. A final 196,000 $\mathrm{g}$ step leads to a separated membrane and to cytosolic fraction as well. Membrane pellets and the corresponding cytosolic supernatant were analysed by Western blotting (left) and immunostained against $\alpha-S y n$. For quantification of membrane binding the ratio of $\alpha$-Syn signal intensity in membrane pellets versus signal intensity in the cytosolic supernatant were determined (left, bottom). $(B+C)$ The blots in $(A)$ were restained with antibodies against GAPDH as a cytosolic marker and against $\beta 5$-Integrin as a membrane marker. All Values are given as mean + SEM from $n=12$ experiments for $\alpha$-Synuclein wild-type, $n=12$ experiments for $2 \mathrm{KR}$ and $n=12$ experiments for 2 AA with $\alpha$-Syn wt normalized to $1 .{ }^{*}$ indicates $p \leq 0.05$, ${ }^{* \star *}$ indicates $p \leq 0.001$; student's 2-side t-test.

Having confirmed that both SUMOylation sites in $\alpha$-Syn at aa 96 and 102 are required for the binding of $\alpha$-Syn to lipid membranes, we designed a myc- $\alpha$-Syn SUMO fusion construct, mimicking SUMO modification and bearing a $\triangle G G$ mutation, which prevents the SUMO conjugation to other proteins and to SUMO itself. After transient transfection, membrane pellets as well as cytosolic supernatants of transfected cells were subjected to Western blotting and probed against $\alpha$-Syn. As shown in Fig. $11 \mathrm{~A}$, membrane binding of an $\alpha$-Syn SUMO fusion protein was markedly increased in N2a cells, compared to the wild-type protein of a-Syn (Fig. 11 B). 
A

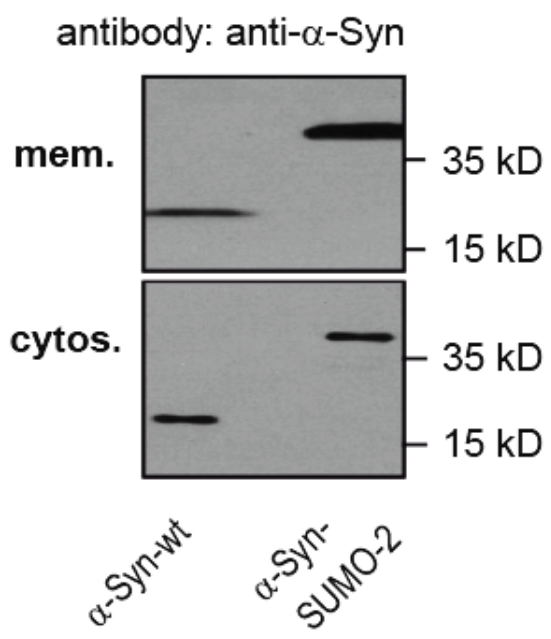

B

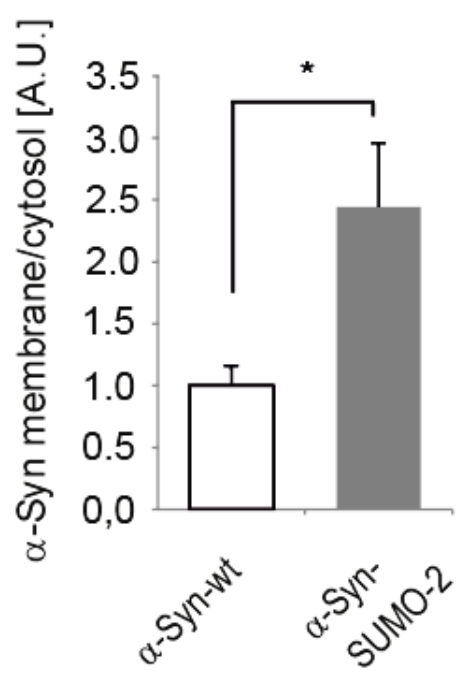

Fig. 11: Membrane binding of a myc- $\alpha-S y n-S U M O$ fusion construct

(A) Plasmids which are either encoding for $\alpha$-Syn wt or for $\alpha$-Syn-SUMO-2 $\Delta$ GG-fusion construct were transfected in cell line N2a. The membrane pellet and the cytosolic supernatant were processed to SDS-PAGE and subsequent analysed by Western blot and membranes were probed with an antibody against $\alpha$-Synuclein. (B) For quantification of membrane binding, the ratio of protein intensity of the membrane pellet versus the corresponding cytotsolic supernatant was determined (right). All values are given as the mean + SEM from $n=6$ experiments, and the mean for $\alpha$-Syn wt was normalized to 1 ; $^{*}$ indicates $p \leq 0.05$; student' 2 -side t-test.

Our collaboration partner Prof. Giese (Dept. of Neuropathology and Prion Research, LudwigMaximilians University Munich) employed fluorescence correlation spectroscopy (FCS)scanning for intensely fluorescent targets (SIFT) (Giese et al. 2005, Högen et al. 2012) as a complementary method, aiming to confirm that the membrane binding propensity of $\alpha$-Syn is regulated by SUMOylation. This method is based on a single particle analysis by adapting a method, derived from fluorescence correlation spectroscopy (Giese et al. 2005). This technique is also used for the efficient analysis of protein aggregation in neurodegenerative diseases, like prion diseases and in Alzheimer's disease (AD) (Schwille et al. 1997, Pitschke et al. 1998, Post et al. 1998, Bieschke et al. 2000, Giese et al. 2000, Giese et al. 2004, Bertsch et al. 2005).

They used a two colour scanning set up with red and green fluorophores and two different excitation lasers together with recombinant $\alpha$-Syn and recombinant sumoylated $\alpha$-Syn (for purification method, see section 2.2.1.5), labelled with Alexa Fluor-647-O-succinimidylester (Giese et al. 2005) which competed for the binding of the green labelled small unilamellar Dipalmitoyl-sn-glycero-3-phospho-choline lipid vesicles (DPPC-SUV) (Högen et al. 2012). 
The frequencies of specific combinations of green and red photon counts were recorded in a two-dimensional (2D) intensity distribution histogram Fig. $12 \mathrm{~A}$. The fluorescence intensity data were calculated by summing up high intensity bins over a defined time period (Fig. $12 \mathrm{~B}$ right panel).

A

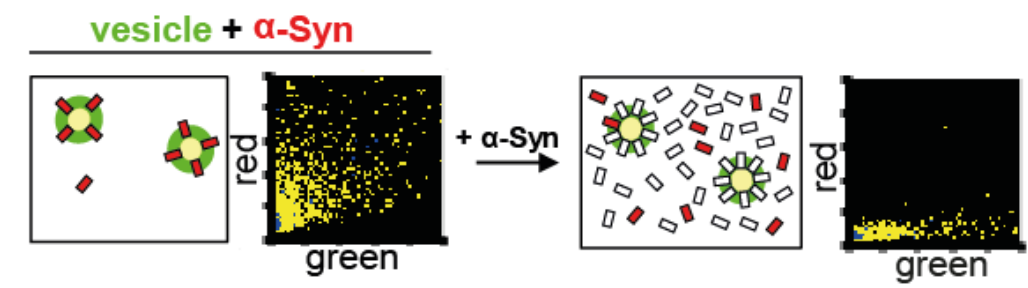

vesicle + sumoylated $\alpha-S y n$

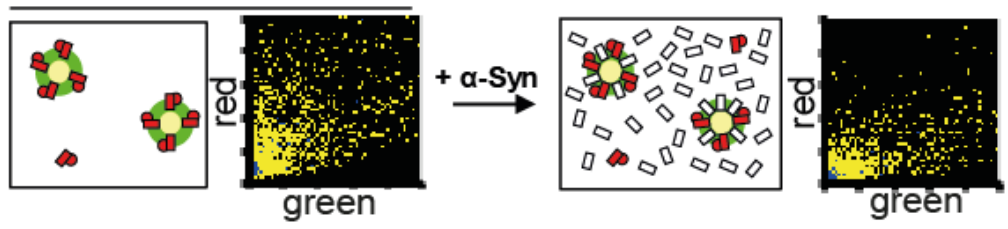

B

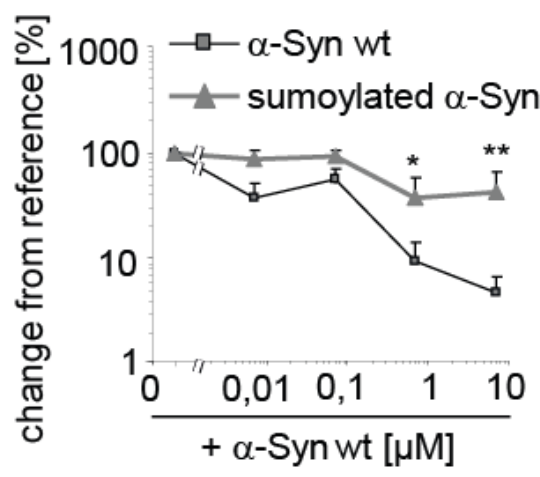

C

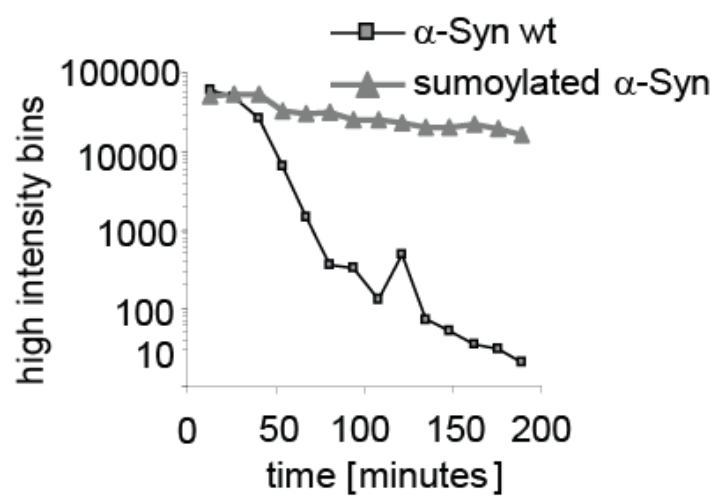

Fig. 12: SIFT assay for vesicle binding properties of sumoylated and non-sumoylated a-Syn

(A) Schematic figure of the assay and two-dimensional fluorescence intensity distribution histograms of SIFT recordings show binding of recombinant $\alpha$-Syn (red bar) and sumoylated $\alpha$-Syn (red bar with red dot) to DPPCSUVs (green circle) and unlabelled non-sumoylated $\alpha$-Syn (white bar). Red fluorescence intensity is given on the vertical axis and the green fluorescence intensity is given on the horizontal axis as photons/bin. (B) Dose response curve for the effect of non-labelled $\alpha$-Syn on the vesicle binding of sumoylated and non-sumoylated $\alpha$ Syn. Values are given as a + SEM normalized to reference (addition of buffer) of duplicate measurements of three parallel samples. (C) Time course of release of $\alpha$-Synuclein and sumoylated $\alpha$-Synuclein after addition of $7 \mu \mathrm{M}$ non-labelled $\alpha$-Syn in a representative experiment.

No change in the distribution of particles could be observed in the absence of unlabelled, non-sumoylated $\alpha$-Syn. In contrast to sumoylated $\alpha$-Syn, non-sumoylated $\alpha$-Syn is released from the lipid vesicles following addition of about 1000-fold excess of unlabelled $\alpha$-Syn (see also Fig. $12 \mathrm{~B}$ left and right panel). 
Following the addition of unlabelled non-sumoylated $\alpha$-Syn, the release of labelled nonsumoylated a-Synuclein from the green labelled DPPC-SUVs, is also visible in the two-dimensional fluorescence intensity distribution histogram (Fig. $12 \mathrm{~A}$ upper right panel) This is in accordance with our results which show increased membrane binding of sumoylated $\alpha$-Syn. Taken these data together one can conclude that SUMOylation of $\alpha$-Syn promotes its binding to (lipid)-membranes.

\subsubsection{Extracellular vesicle release of $\alpha$-Synuclein is regulated by SUMOylation}

To investigate whether SUMOylation might have an influence on the release of $\alpha$-Syn within EVs, we determined the EV secretion of both SUMOylation deficient $\alpha$-Syn mutants. To address this issue, N2a cells were transiently transfected with both SUMOylation deficient mutants of $\alpha$-Syn. After an expression time for all constructs of $8 \mathrm{~h}$, we changed the medium from DMEM (see section 2.1.7.2) with fetal calve serum, to medium without serum and collected EVs $16 \mathrm{~h}$ (see section 2.2.2.5). Collecting medium was subjected to subsequent centrifugation steps, including a final ultracentrifugation step, to pellet down EVs (see section 2.2.3.1). After preparation of EVs we subjected the P100 and the corresponding parental cell lysate to SDS-PAGE and subsequently to Western blot analysis. The membranes were probed for a-Syn and as a control for the EV marker protein Flotilin-2. As shown in Fig. 13 A, we were able to detect $\alpha$-Syn in the EV fraction and in the lysates. Both SUMOylation deficient mutants were significantly reduced in the EV fraction, compared to $\alpha$-Syn wt (Fig. $13 \mathrm{~B}$ ). With nanoparticle tracking analysis (NTA), we investigated, whether the transfection of the different mutant versions of $\alpha$-Syn, might change the total number of EVs released by the neuroblastoma cell line N2a. With this technique it is possible to analyse nanoparticles in real time. To determine the number of EVs, $200 \mu \mathrm{L}$ of cultured medium were taken and centrifuged for $5 \mathrm{~min}$ at $5.000 \mathrm{rpm}$. The supernatant was diluted 1:1 with PBS. NTA was performed with a NanoSight LM14 instrument, which consists of a conventional optical microscope with a high resolution camera, which uses a $532 \mathrm{~nm}$ laser to illuminate the nanoparticles. During the analysis the particles were illuminated by the laser beam, which results in Brownian motion of the illuminated particles.

The Brownian motion of the particles were then recorded by a high resolution camera and the analysis software of the device allows for an automatic tracking of these particles and determines both, the particle concentration and the size distribution of the recorded particles. In order to determine the concentration of released vesicles, we recorded 3 videos with duration of 30 s and a camera level of 11 , for each construct. 
The detection threshold was set at 10 and at least 800 tracks were analysed for each video. The concentration of vesicles smaller than $120 \mathrm{~nm}$ was analysed, all larger vesicles were excluded from the analysis. We found no significant differences in the release of EVs between $\alpha$-Syn wt and both SUMOylation deficient mutants (see Appendix, Table 13). This indicates that expression of $\alpha$-Syn or its mutants does not interfere with the number of released vesicles.

A

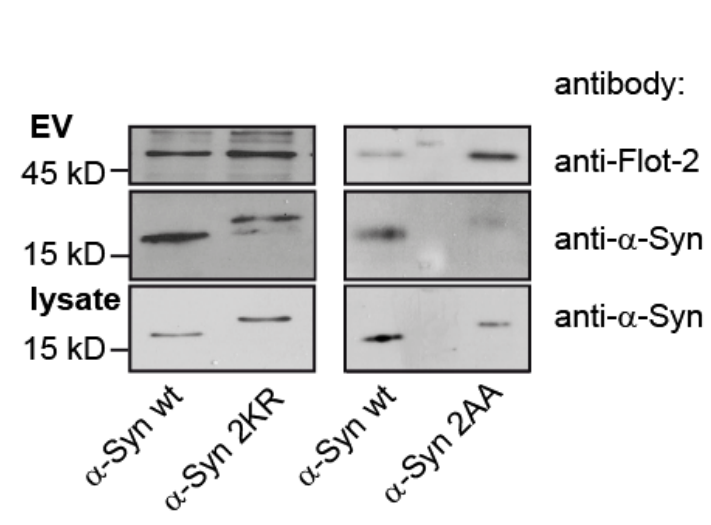

B

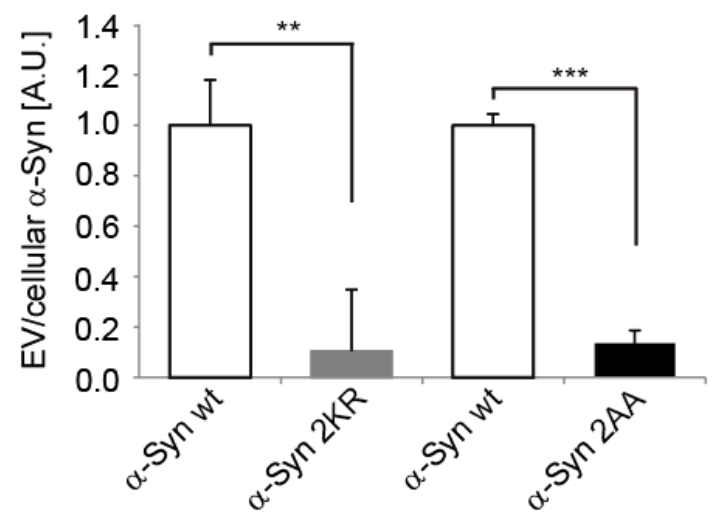

Fig. 13: Extracellular vesicle release of SUMOylation deficient $\alpha$-Syn

(A) Western blot analysis of EVs and the corresponding parental cell lysate from N2a cells transfected with both sumoylation deficient mutants (2 AA and $2 \mathrm{KR}$ ) and $\alpha$-Syn wt as well. Blots were scanned and the signal intensities of the bands were determined by ImageJ software analysis. (B) The quantification histogram shows the ratio of $\alpha$-Syn wt (white bars left and right) which was normalized to 1, and the $\alpha$-Syn mutant 2 KR (grey bar) and the a-Syn mutant $2 \mathrm{AA}$ (black bar) intensities in EV pellets versus corresponding cell lysates. The EV release of both SUMOylation deficient mutants was impaired compared to a-Syn wt. The membranes of the EV fractions were additionally probed with an antibody against Flotilin-2, as an EV marker protein. All values are given as mean + SEM from $n=6$ independent experiments; ${ }^{* *}$ indicates $p<0.01,{ }^{* * *} p<0.001$, student's 2 -side t-test.

Additionally, primary cortical neurons were infected with an adeno-associated virus to express either $\alpha$-Syn wt or the SUMOylation deficient mutant $\alpha$-Syn 2 KR (Krumova et al. 2011). After 4 days of post-infection the cultured medium was collected and further processed to EV preparation as described in section 2.2.3.1 (notably, for this approach the P-100 was not resuspended in protein loading buffer, but rather in CHAPS lysis buffer (see section 2.1.6.5)) to further quantify the amount of $\alpha$-Syn in the EV fraction and in the corresponding cell lysate by an electrochemiluminescence assay (Kruse et al. 2012). 


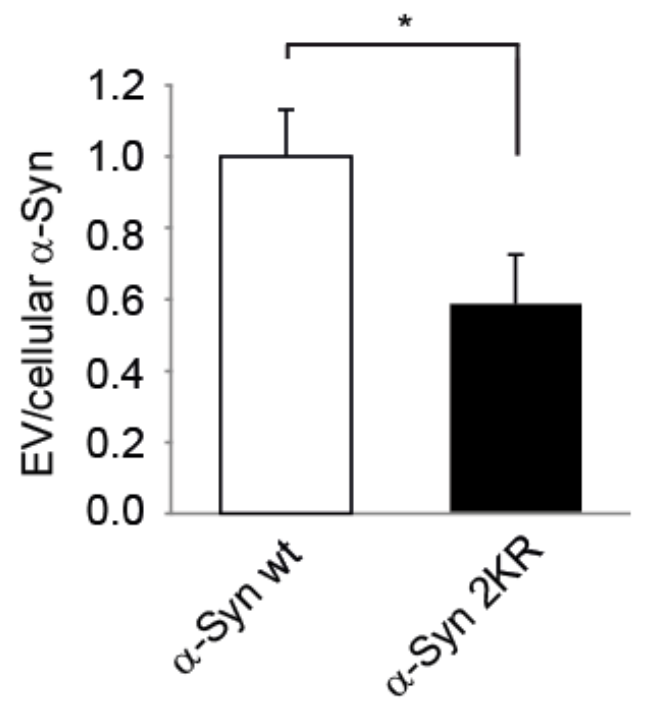

Fig. 14: Primary cortical neurons were infected with AAV to either express $\alpha$-Syn-wt or the SUMOylationdeficient mutant aSyn-2 KR

Extracellular vesicles were prepared from cultured medium of primary cortical neurons and the amount of $\alpha$-Syn was quantified in EV fractions and in the parental cell lysates by an electrochemiluminescence assy. The quantification histogram shows the calculated ratio of EVs versus cellular $\alpha$-Syn for wild-type (white bar) which was normalized to 1, and the $\alpha$-Syn mutant $2 \mathrm{KR}$ (black bar). All values are given as mean + SEM from $n=15$ independent experiments. * indicates $p<0.5$; student's 2 -side t-test.

The assay was performed in collaboration with Prof. Brit Mollenhauer and Dr. Niels Kruse, Dept. of Neuropathology, University Medical Center Göttingen. As shown in Fig. 14 we were able to detect a significant reduction of extracellular release of $\alpha$-Syn $2 \mathrm{KR}$ mutant compared to $a-S y n$ wt. In conclusion, this data from primary neurons confirms our findings obtained in the neuroblastoma cell line N2a. The results show that SUMOylation increases EV of $\alpha-S y n$.

\subsubsection{Silencing of Ubc9 decreases the release of $\alpha$-Synuclein within extracellular $\underline{\text { vesicles }}$}

To further prove our conclusion that SUMOylation increases EV release of a-Syn, we silenced the SUMO E2 conjugating enzyme Ubc9 (UBE2I) by RNA interference and assessed its effect on the secretion of a-Syn in EVs. E2 enzymes are able to catalyse the attachment of ubiquitin and ubiquitin-like proteins (e.g. SUMO) to acceptor lysines of other proteins. This reaction is mediated directly or via specific E3 enzymes (Bernier-Villamor et al. 2002). Human embryonic kidney cells (HEK), were either treated with Ubc9 siRNA or mock treated for 36 hours. After 36 hours the cells were harvested. The cell lysate was subjected to Western blotting and the membrane was probed with antibodies against Ubc9 and Actin as a loading control to quantify the down regulation of Ubc9. 
As presented shown in Fig. $15 \mathrm{~A}$, Ubc9 protein levels were significantly decreased in the cells treated with siRNA against Ubc9 compared to mock treatment. To quantify the knockdown efficiency of the Ubc9 siRNA, we calculated the ratio of $\alpha$-Syn to Actin protein levels. We normalized the ratio of Mock treated cells to 1 . The quantification revealed a knockdown efficiency of approximately $80 \%$ for cells treated with siRNA against Ubc9 (Fig. $15 \mathrm{~B})$. To determine the effect of Ubc9 down-regulation on EV release of $\alpha$-Syn, HEK cells were treated with Ubc9 siRNA or mock treated 36 hours prior to transfection with $\alpha$-Syn wt. After 8 hours post-transfection time, the cells were washed with PBS and the medium was changed to medium without FCS, to collect EVs for 16 hours. The EV containing medium was collected and purified by ultracentrifugation. The resulting pellets P100 and the corresponding cell lysates were subsequently analysed by western blot analysis with antibodies against $\alpha$-Syn and Alix. Indeed, Ubc9 RNAi resulted in a significantly decreased secretion of $\alpha$-Syn within EVs (Fig. $15 \mathrm{C}+\mathrm{D}$ ). The total number of EVs was unaltered by the siRNA treatment, as indicated the by EV marker protein Alix (Fig. $15 \mathrm{C}$, upper panel). 
A

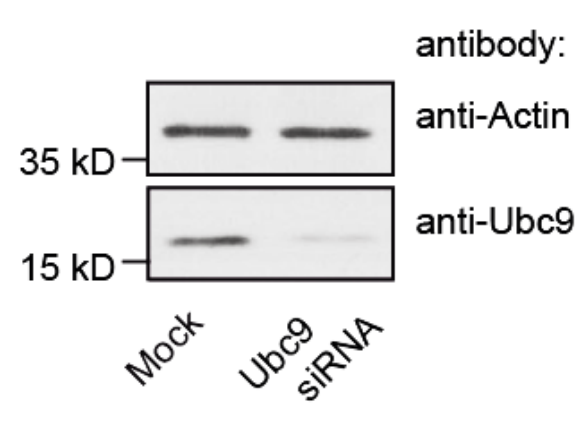

C

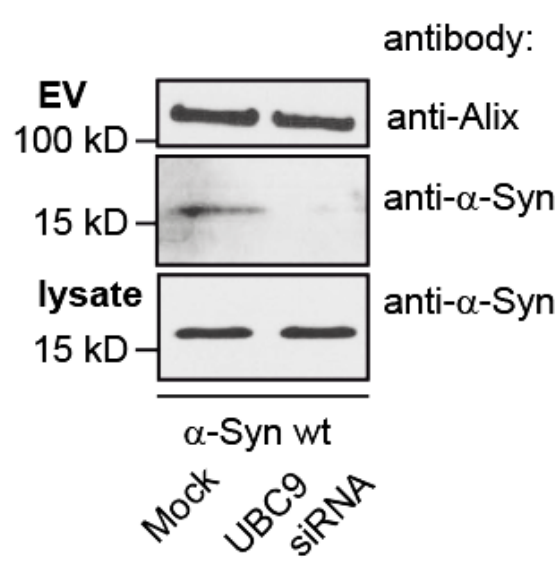

B

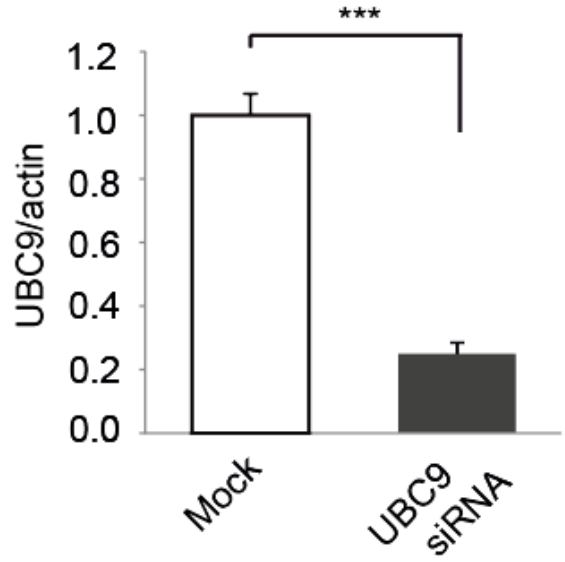

D

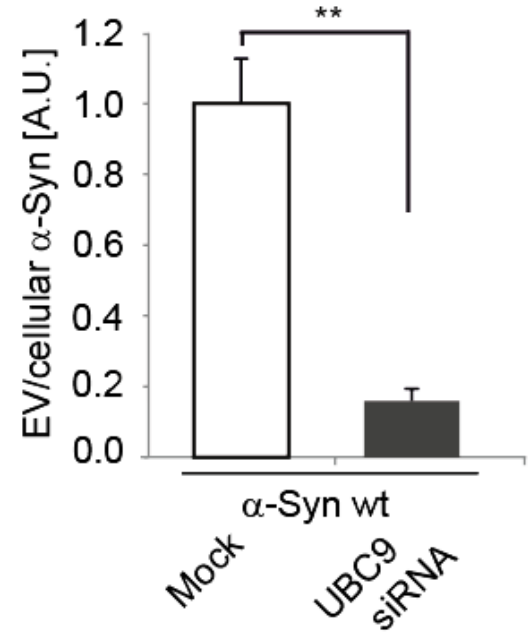

Fig. 15: Down-regulation of Ubc9 protein levels with siRNA

(A) Efficiency of siRNA-mediated down-regulation was quantified by Western blot analysis of cell lysates. Membranes were immunostained with antibodies against Ubc9 and Actin. (B) For the quantification of silencing efficiency, the ratio of Ubc9 to Actin was calculated. The silencing efficiency was around $80 \%$. Results are given as mean + SEM, student's t-test with $n=8$ individual experiments, ${ }^{* * *} \mathrm{p}<0.0005$. (C) Western blot analysis of Ubc9 siRNA and Mock treated HEK cells. Membranes were immunostained with antibodies against $\alpha$-Syn and Alix as a positive control for the purity of EV preparations. (D) The ratio of EV to cellular $\alpha$-Syn was determined by calculating a ratio between Mock (white bar) and siRNA (grey bar) treated cells. All results are given as mean + SEM, ** indicates $p<0.005$; Mock was normalized to $1 ; 2$-side students t-test with $n=6$. 


\subsubsection{2. $\quad \underline{\alpha-S y n u c l e i n ~ f u s i o n ~ w i t h ~ S U M O-2 ~ i n c r e a s e s ~ t h e ~ r e l e a s e ~ o f ~} \alpha$-Synuclein within} extracellular vesicles

To investigate, whether increased SUMOylation would promote EV release of a-Syn, N2a cells were transfected either with myc- $\alpha$-Syn-wt or with myc- $\alpha$-Syn-SUMO-2, mimicking constitutive SUMO modification. We then harvested the cell lysates and prepared EVs from the culture medium, which were subjected to SDS-PAGE and for Western blot analysis. We found that EV release of $\alpha$-Syn-SUMO-2 was increased compared to $\alpha$-Syn wt (Fig. $16 \mathrm{~A}$ and B). NTA analysis revealed no significant difference in the amount of secreted EVs in both conditions (see Appendix, Table 13).

A

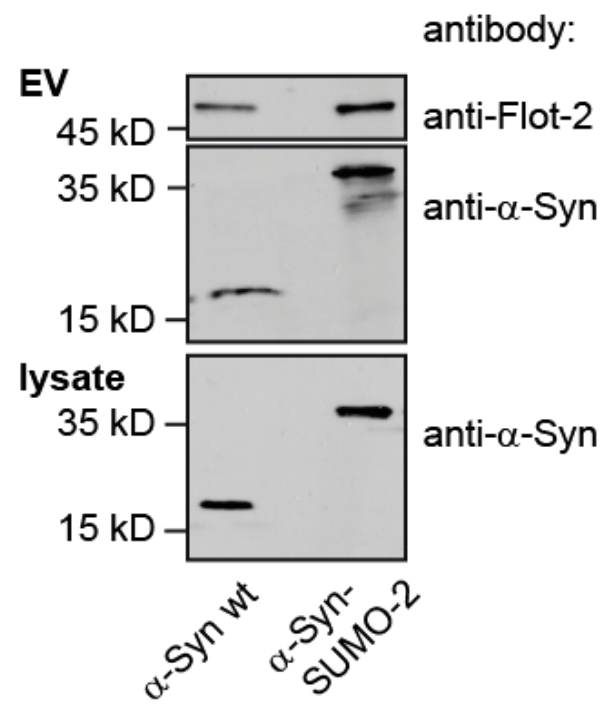

B

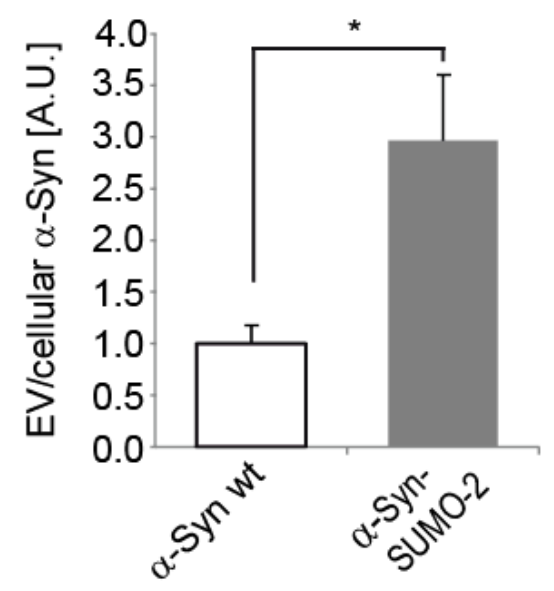

Fig. 16: SUMO-2 fusion increases extracellular vesicle release of $\alpha-S y n$

(A) EVs and corresponding cell lysates were analysed by Western blotting and immunostained with an antibody against $\alpha$-Syn. The membranes of the EV fractions were additionally probed with an antibody against Flotilin-2 as an EV marker protein. (B) For quantification of EV release, the signal intensity for $\alpha$-Syn in the EV fraction, versus the signal intensity for $\alpha$-Syn in the parental cell lysate was determined. The ratios were normalized to the wt and set to1. All values are given as mean + SEM from $n=8$ independent experiments; ${ }^{*}$ indicates $p \leq 0.05$, in student's 2-side t-test. 


\subsubsection{3. $\quad$ Co-expression of SUMO-2 increases the release of $\alpha$-Synuclein within} extracellular vesicles

In a slightly different approach we co-expressed a-Syn with either wt SUMO-2 or a conjugation deficient SUMO-2 $\triangle G G$ mutation. EV pellets as well as parent cell lysates of cotransfected cells were subjected to Western blotting and the membranes were probed with an antibody against $\alpha$-Syn (Fig. 17 A). Co-transfection of myc- $\alpha$-Syn wt together with mycSUMO-2 wt significantly increased the amount of $\alpha$-Syn in EVs compared to co-expression of the conjugation-deficient SUMO mutant myc-SUMO-2 $\triangle \mathrm{GG}$ (Fig. $17 \mathrm{~B}$ ).

A

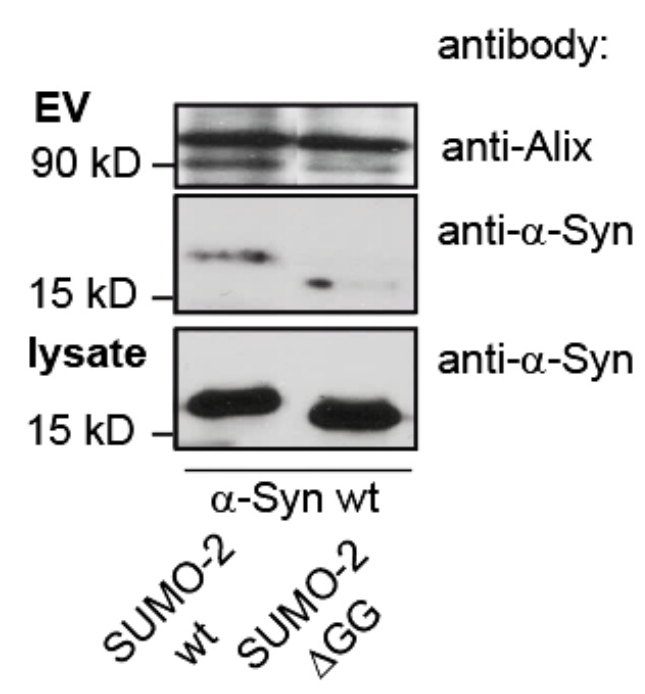

B

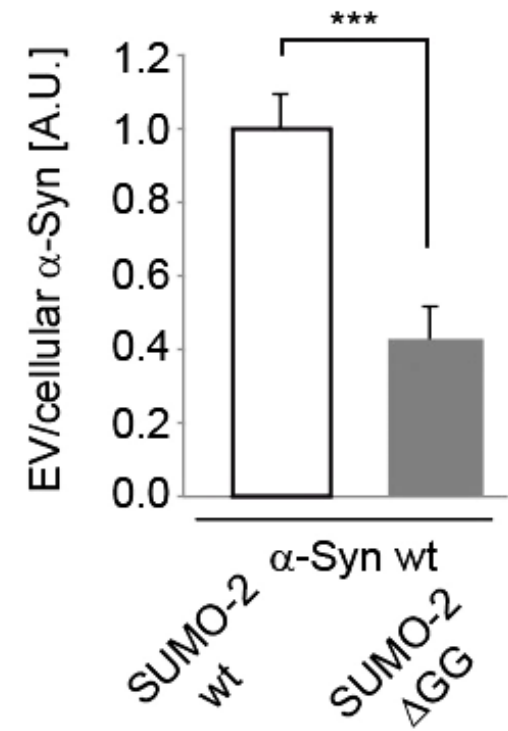

Fig. 17: Co-expression of SUMO-2 increases release of $\alpha$-Syn with extracellular vesicles

(A) N2a cells were transiently co-transfected with plasmids either encoding for the mature SUMO-2 version (mycSUMO-2 wt) or for the conjugation deficient mutant myc-SUMO-2 $\triangle G G$. Extracellular vesicles were prepared and analysed together with the parental cell lysates by SDS-PAGE und were further processed to Western blot analysis. The EV fraction and the cellular fraction were immunostained against $\alpha$-Syn and for quantification of signals subjected to signal intensity analysis via ImageJ software. (B) The histogram displays the calculated ratio between signal intensity of the extracellular fraction versus the corresponding cell lysate. (SUMO-2 wt is normalized to 1) All values are given as mean + SEM from $n=10$ independent experiements. ${ }^{* * *}$ indicates $p \leq$ 0.001 ; student's 2-side t-test.

By NTA analysis, no significant difference was observed in the amount of EVs by SUMO-2wt or SUMO-2- $\triangle$ GG overexpression (see appendix, Table 13). Likewise, WB analysis of Flotillin-2 and Alix in the EV fractions revealed no differences between SUMO-2-wt and SUMO-2- $\triangle G G$ mutant co-expression (Fig. 18). This data indicates that SUMO expression does not increase the release of EVs per se but specifically the release of $\alpha$-Syn with EVs. 
A

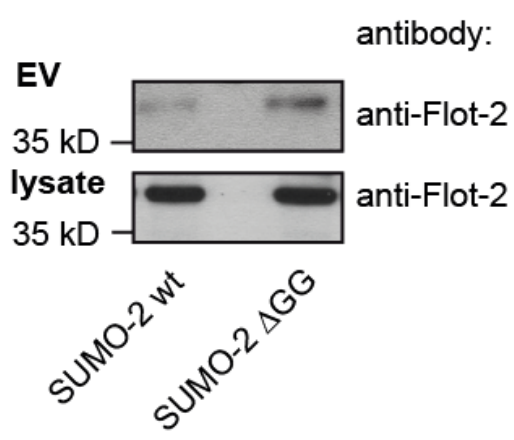

C

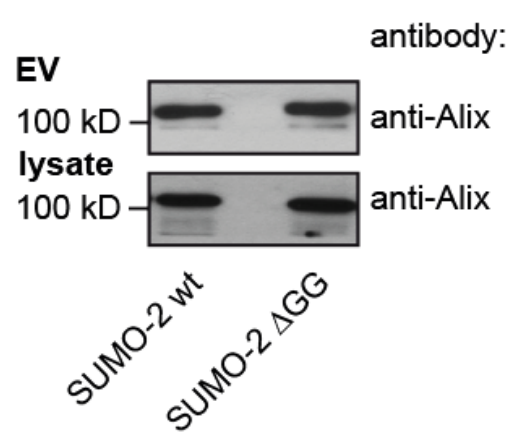

B

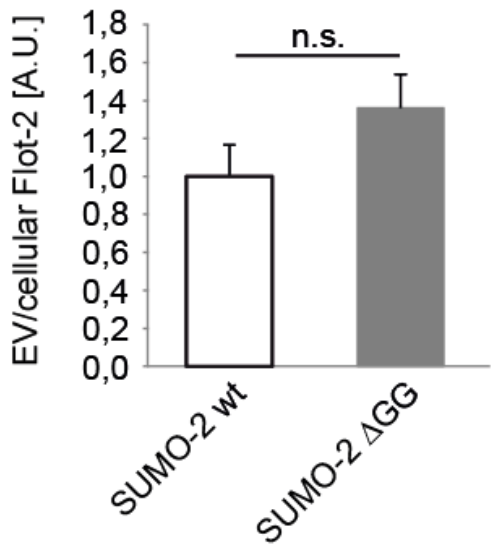

D

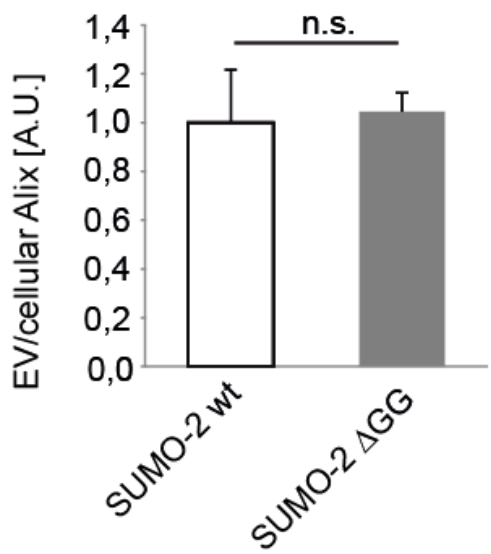

Fig. 18: Co-expression of SUMO-2 does not increase the production and release of extracellular vesicles itself

The neuroblastoma cell line N2a was co-transfected with $\alpha$-Syn wt and either myc-SUMO-2 wt or the conjugation deficient mutant myc-SUMO-2 $\triangle$ GG. (A) EVs and the corresponding cell lysates were prepared and processed by Western blot analysis. Membranes were probed with an antibody against Flotilin-2. (B) The histogram shows the ratio of Flotilin-2 signal intensities of EV pellets versus cell lysates, of myc-SUMO-2 wt (white bar) and the conjugation deficient mutant myc-SUMO-2 $\triangle G G$ (grey bar). (C) Western blot analysis of EV pellets and the parental cell lysates that were stained with an antibody against Alix. The ratios of Alix signal intensities in the EV fraction versus cellular fractions were calculated. (D) The histogram displays no significant difference for the release of Alix positive EVs, when $\alpha$-Syn was either co-transfected with myc-SUMO-2 wt (white bar), or the mutant myc-SUMO-2 $\triangle G G$ (grey bar). All values are given as mean + SEM of $n=6$ independent experiments; n.s. = not significant; student's 2-side t-test.

In summary, our data show that membrane binding is required for EV release of $\alpha$-Syn. SUMOylation of $\alpha$-Syn increases membrane binding and also EV release, whereas SUMO deficient mutants of $\alpha$-Syn show less membrane binding and decreased EV release (Fig. 19). 


\begin{tabular}{|l|l|l|}
\hline membrane binding deficient & membrane binding & release with EVs \\
\hline $\begin{array}{l}\alpha \text {-Syn wt } \\
\alpha \text {-Syn } \Delta \mathrm{N}\end{array}$ & ++ & ++ \\
\hline sumoylation deficient & & - \\
\hline $\begin{array}{l}\alpha \text {-Syn wt } \\
\alpha \text {-Syn 2AA }\end{array}$ & ++ & ++ \\
$\alpha$-Syn 2KR & - & - \\
\hline SUMO fusion & - & - \\
\hline $\begin{array}{l}\alpha \text {-Syn wt } \\
\alpha \text {-Syn-SUMO-2 }\end{array}$ & ++ & ++ \\
\hline SUMO co-transfection & & +++ \\
\hline $\begin{array}{l}\alpha \text {-Syn wt + SUMO-2 wt } \\
\alpha \text {-Syn wt + SUMO-2 } \Delta \text { GG }\end{array}$ & +++ & +++ \\
\hline
\end{tabular}

Fig. 19: Summary of $\alpha$-Syn membrane binding and release with extracellular vesicles

Schematic summary of $\alpha$-Syn membrane binding (middle column) and release within extracellular vesicles (right column). 


\subsubsection{Isopeptidase acitivity in extracellular vesicles results in a rapid de-conjugation of}

\section{SUMO}

We could not detect sumoylated- $\alpha-S y n$ in EVs by Western blots analysis. It is known that SUMO modification is transient and can be rapidly removed by SUMO specific proteases (Shin et al. 2012).

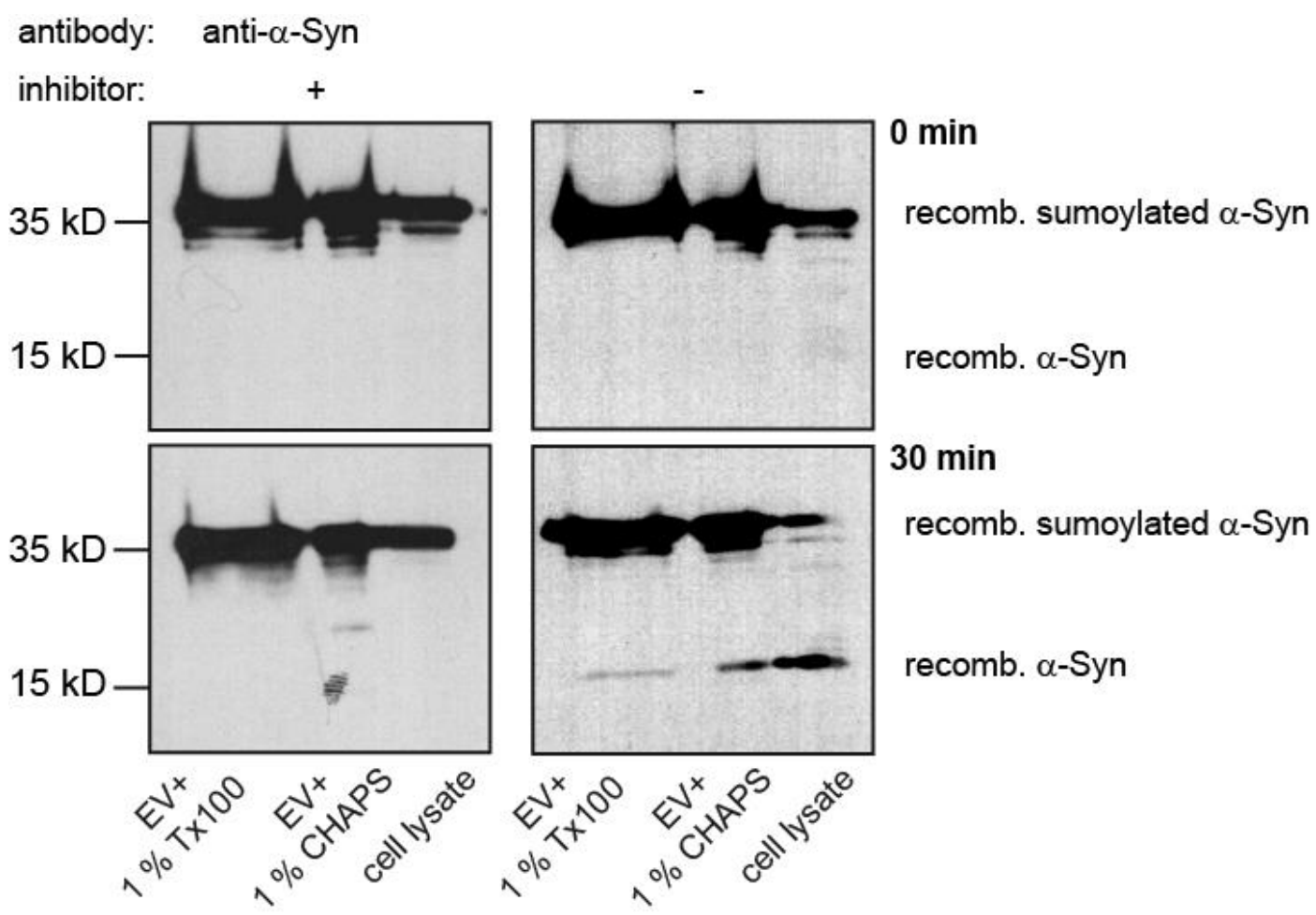

\section{Fig. 20: Extracellular vesicles contain desumoylase activity}

N2a cells were cultured for $24 \mathrm{~h}$ and the medium was changed to medium without FCS to collect EVs. After $24 \mathrm{~h}$ EVs were prepared and the corresponding cell lysates were prepared by scraping in $1 \%$ CHAPS buffer. The vesicles were lysed with either $1 \%$ Triton X 100 or $1 \%$ CHAPS in the presence (left) or in the absence (right) of $\mathrm{N}$-Ethylmaleimide, which is known to inhibit de-sumoylases (isopetidases). Lysed EVs and cell lysates were incubated for $0 \mathrm{~min}$ (top panel) or $30 \mathrm{~min}$ (right) panel at $37^{\circ} \mathrm{C}$ with recombinant sumoylated $\alpha$-Syn. The reaction was stopped by adding protein loading buffer. All samples were subjected to SDS-PAGE und subsequently analysed by Western blot with an antibody against $\alpha$-Syn. One representative experiment, out of $n=3$ is shown.

The enzymes responsible for the de-conjugation of SUMO in mammals include two ubiquitinlike-specific proteases in yeast, named Ulp1 and UIp2 and six sentrin-specific proteases (SENP1, 2, 3, 5, 6 and 7) (Hay 2007, Yeh 2009). All members of the SENP and both Ulp proteases belong to the C48 family of cysteine proteases, by sharing a conserved catalytic His-Cys-Asp triad (Schulz et al. 2012). To investigate, whether an isopeptidase (de-sumoylase) activity in EVs results in de-conjugation of SUMO within EVs, we prepared EVs from N2a cells. 
The prepared vesicles were either lysed in $1 \%$ CHAPS or $1 \%$ Triton X-100. The lysed vesicles and $\mathrm{N} 2 \mathrm{a}$ cell lysate serving as a positive control were then incubated with recombinant sumoylated $\alpha-S y n$ at $37^{\circ} \mathrm{C}$ for 0 or $30 \mathrm{~min}$ in the presence or absence of $20 \mathrm{mM}$ $\mathrm{N}$-ethylmaleimide (NEM). NEM inhibits isopeptidases by forming a stable, covalent thioether bond with cysteine residues. The reaction was stopped by adding protein loading buffer (see 2.1.6.6) and the samples were subjected to Western blot analysis and probed with an antibody against $\alpha$-Syn. After 0 min of incubation no de-sumoylated $\alpha$-Syn was detected, neither in the presence or absence of NEM. After an incubation period of $30 \mathrm{~min}$ de-sumoylated $\alpha$-Syn appeared in the absence of NEM while in the presence of NEM only sumoylated $\alpha$-Syn was detectable. Taken together, we found an isopeptidase activity in EVs, which results in a rapid cleavage of SUMO from a-Syn (Fig. 20).

Since we were unable to detect sumoylated $\alpha$-Syn by WB in EVs, we used a luciferasebased protein fragment complementation assay (Danzer et al. 2012), to detect sumoylated a-Syn in EVs. We used a bioluminescence protein-fragment complementation assay (Outeiro et al. 2008, Tetzlaff et al. 2008, Putcha et al. 2010). For this assay a-Syn was fused to full length Gaussia princeps luciferase (Remy et al. 2006) (syn phGluc), or to the amino-terminal or carboxy-terminal fragments of split phGluc ( $\alpha$-Syn-S2) or SUMO-2 (SUMO-2 S3). Close proximity of SUMO and $\alpha$-Syn will result in complementation of split luciferase which can be quantified by luminescence. 


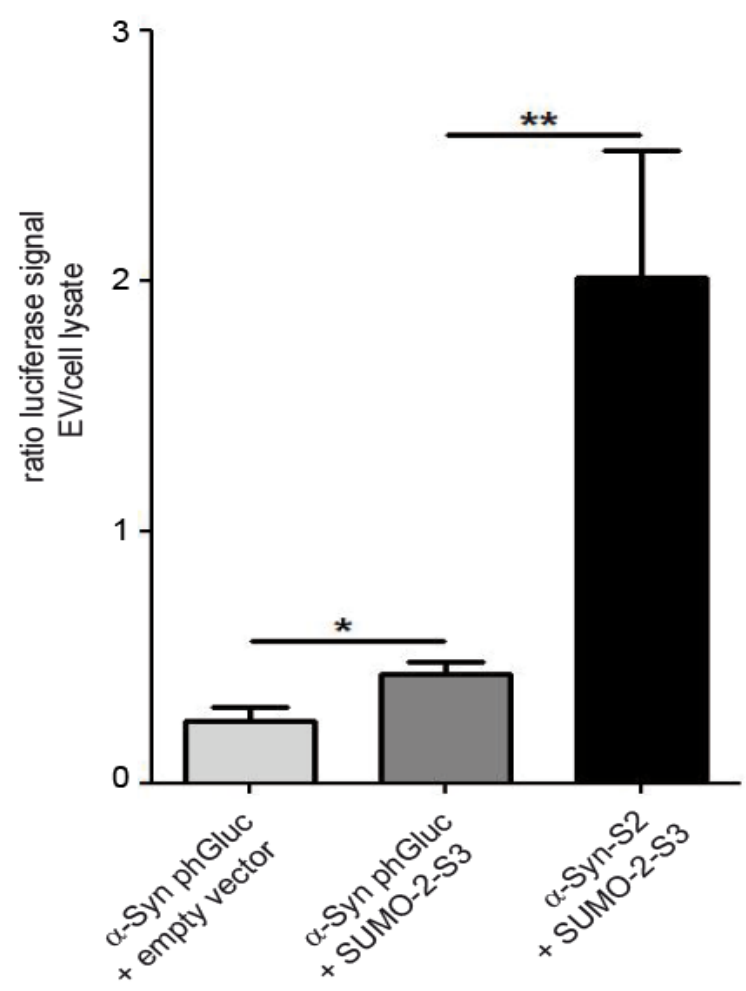

Fig. 21: Sumoylated $\alpha-S y n$ is enriched in extracellular vesicles

Constructs indicated above were transfected into HEK 293 cells. Cells were washed after $16 \mathrm{~h}$ post-transfection and PBS was replaced with serum- and phenol free media. After $48 \mathrm{~h}$ the medium was collected, EVs and cell lysates were prepared. Luciferase activity from protein complementation was measured using the same amount of total protein of the cell lysates and EV fractions. The ratio of luciferase activity signal was calculated for the EV fraction versus cell lysates. The histogram shows significant increase in luciferase activity when $\alpha$-Synuclein fused to full length gaussia luciferase was co-expressed with SUMO-2 (dark grey bar) compared to the control, expressing only the aS-full length gaussia luciferase construct (light grey bar). The highest luciferase signal was obtained when C- or N-terminal fragments of split gaussia luciferase were fused to $\alpha$-Synuclein ( $\alpha$-Syn-S2) or to SUMO-2 (SUMO-2-S3) (black bar). All values are given as mean + SEM of $n=3$ independent experiments; student's 2-side t-test; * indicates $p \leq 0.05$ and ${ }^{* *}$ indicates $p \leq 0.01$. Measurements and data analysis were performed by Marisa Feiler, Karin M. Danzer (Dept. of Neurology, Ulm University, Germany)

To address the question whether sumoylated a-Syn is enriched in EVs, we transfected either (a) $\alpha$-Syn coupled to luciferase, (b) a-Syn coupled to luciferase plus Sumo-2 split luciferase or (c) a-Syn coupled to split luciferase plus SUMO-2 coupled to split luciferase into HEK 293 cells. EVs were prepared and cells were washed with PBS and lysed by sonication. The probes were subjected to luciferase measurements in an automatic plate reader at $480 \mathrm{~nm}$. As shown in Fig. 21 only a low luciferase signal was obtained in EVs when a-Syn was fused to the full length construct of Gaussia luciferase (light grey bar). In contrast, co-transfection of a-Syn fused to full length Gaussia luciferase ( $\alpha$-Syn-phGluc), co-expressed with SUMO-2 (SUMO-2-S3), resulted in a significantly increased luciferase activity signal (dark grey bar). These findings indicate that $\alpha-S y n$ is targeted to EVs, when sumoylated to a higher degree. 
In a similar fashion, when C-or N-terminal fragments of split luciferase were fused to $\alpha$-Syn (a-Syn-S 2) or SUMO-2 (SUMO-2-S 3) and co-transfected into HEK 293 cells, only a-Syn which was modified by SUMO-2, resulted in a dramatic increase of luciferase signal (black bar). These findings indicate that sumoylated $\alpha$-Syn is present and also enriched in EVs.

\subsection{SUMOylation can act as sorting signal for the release within extracellular vesicles}

Next, we wanted to clarify whether SUMOylation acts as a sorting signal for release within EVs. To answer this question we designed a GFP-SUMO-2- $\triangle G G$ fusion construct and as a positive control a GFP-Ub- $\Delta G G$ construct, both constructs bearing, as described above, the $\triangle G G$ mutation to prevent the conjugation of SUMO or Ubiquitin (Ub) to other proteins or themselves. We decided to use ubiquitin as a positive control, because it is known that mono-ubiquitination (Hicke et al. 2003, Haglund et al. 2005, Duncan et al. 2006, Huang et al. 2006) directs cargo for EV release. As a negative control we used GFP because as a cytosolic protein, GFP is excluded from extracellular vesicle release. We prepared EVs and corresponding cell lysates according to the previously described protocols (see section 2.2.3.1) from cultured medium of N2a cells. For further analysis we subjected cell lysates and EV fractions to SDS-PAGE and subsequently to Western blot analysis. The membranes were probed with an antibody against GFP. As shown in Fig. 22 A, GFP was nearly absent from the EV fraction GFP signal intensity was normalized to 1. Results of the quantification are displayed in the histogram in Fig. $22 \mathrm{~B}$ (right) indicating that the release of the GFP-Ub fusion protein within EVs (positive control) was increased up to 16-fold compared to GFP. In a similar fashion, the GFP-SUMO-2 fusion protein was released within EVs with an increase up to 6 -fold compared to GFP, but to a lesser extend when compared to the GFP-Ub fusion protein. 
A
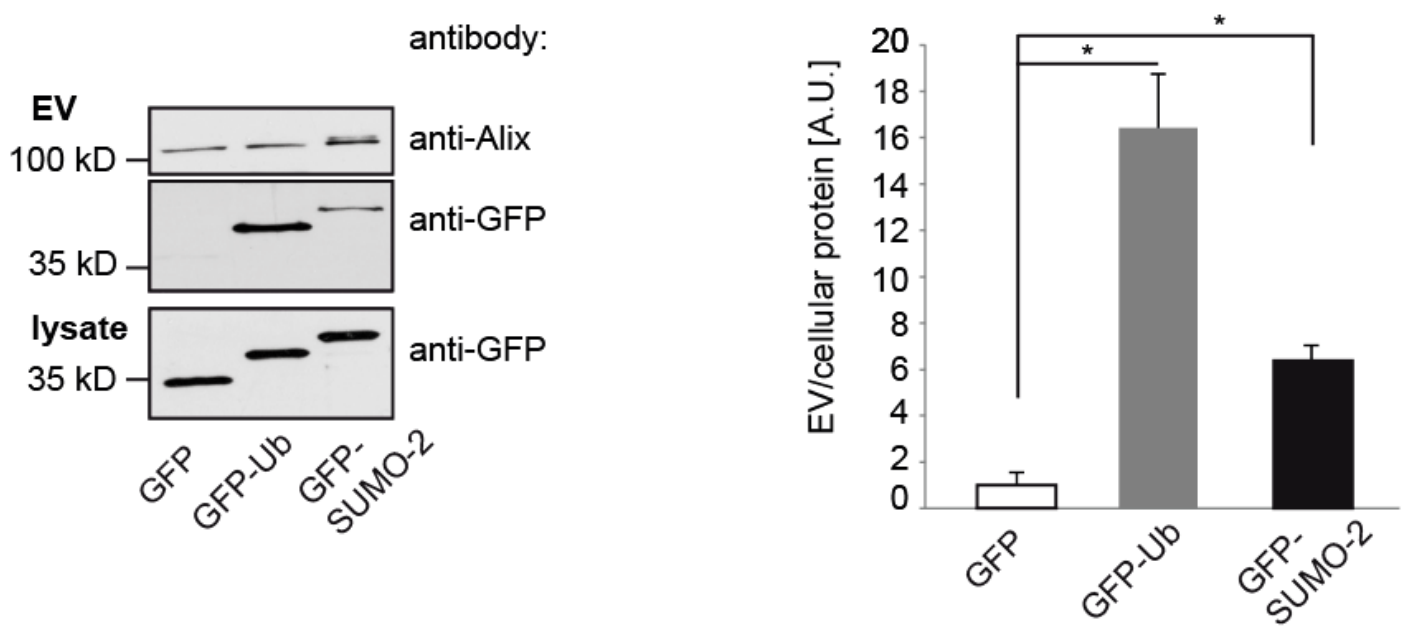

C

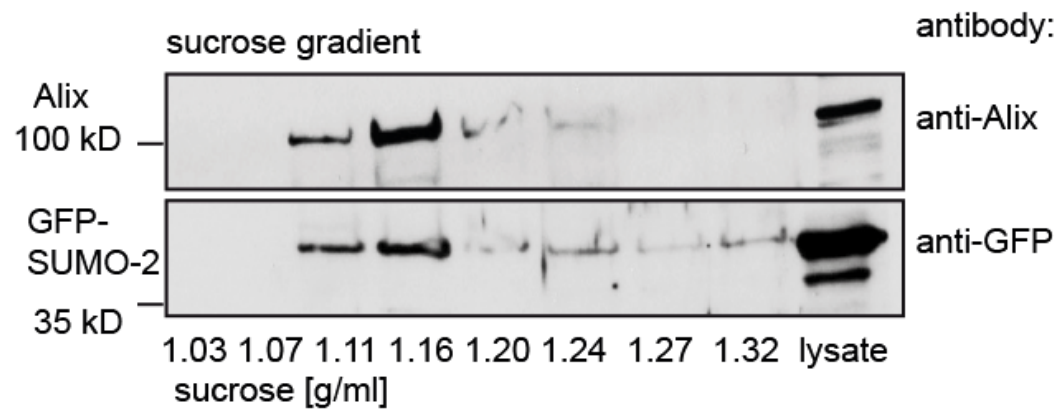

\section{Fig. 22: SUMO-2 is released within extracellular vesicles}

N2a cells were transiently transfected with GFP or GFP either fused to a conjugation deficient Ubiquitin mutant (GFP-Ub $\triangle G G$ ) or to the conjugation deficient SUMO-2 mutant (GFP-SUMO-2 $\Delta G G$ ). (A) EVs and the parental cell lysates were prepared and subjected to SDS-PAGE and further analysed by Western blotting. The membranes were probed with an antibody against GFP and the EV protein Alix, as an internal loading control. (B) The blots were scanned and analysed with ImageJ software, to calculate the ratios of GFP signal intensities in the EV fraction versus cellular fractions. The histogram displays an increase of EV release of GFP-Ub $\triangle G G$ (grey bay) up to 16 -fold compared to GFP (white bar) and an increase of GFP-SUMO-2 $\Delta$ GG (black bar) up to 6 -fold when compared to GFP. All values are given as mean + SEM of $n=8$ independent experiments; student's 2-side t-test; * indicates $p \leq 0.05$. (C) N2a cells were transiently transfected with a construct encoding for the GFPSUMO-2 fusion protein. EVs were prepared as described previously and the EV pellet was loaded for further purification on top of discontinuous sucrose gradient with a range of $1.03 \mathrm{~g} / \mathrm{mL}$ to $1.32 \mathrm{~g} / \mathrm{mL}$. The gradient was centrifuged for $16 \mathrm{~h}$ at 200,000 $\times \mathrm{g}$ and the 8 different layers were diluted 1:6 in PBS and re-centrifuged again at $100,000 \times \mathrm{g}$. The obtained pellets and one representative cell lysate were analysed by Western blot and membranes were stained against GFP and Alix.

To verify that SUMO -2 GFP is sorted into EVs, we additionally performed a sucrose gradient centrifugation. The P100 pellet was resuspended in $0.25 \mathrm{M}$ sucrose and loaded on top of a discontinuous sucrose density gradient $0.25 \mathrm{M}-2.5 \mathrm{M}$ sucrose $(1.03 \mathrm{~g} / \mathrm{mL}-1.32 \mathrm{~g} / \mathrm{mL})$. After centrifugation at $200,000 \times g$ for $16 \mathrm{~h}, 8$ fractions were collected corresponding to the densities indicated above, diluted 1:6 with PBS and subsequent re-centrifuged at 100,000 $\mathrm{g}$ for $1 \mathrm{~h}$. 
For further analysis the 8 fractions were subjected to Western blotting and blot membranes were probed with an antibody against GFP and additionally against the EV marker protein Alix as a control. As presented in Fig. $22 \mathrm{C}$, we were able to detect GFP-SUMO-2 $\Delta G G$ positive exosomes floating at a density of $1.11-1.16 \mathrm{~g} / \mathrm{mL}$. A similar floating behaviour was observed for the EV marker protein Alix as shown in Fig. $22 \mathrm{C}$ upper panel, which is consistent with previously described floating behaviour for EVs on sucrose gradients (Fauré et al. 2006, Théry et al. 2006).

\subsubsection{SUMO-2 targets the cytosolic protein GFP to extracellular vesicle release}

Next, we wanted to rule out the unspecific sorting of GFP-SUMO-2 into EVs mediated by the GFP-fusion. Thus, we transiently transfected N2a cells with SUMO-2 either fused to a GFPor a myc-tag. We prepared EVs and parental cell lysates as described previously in this thesis and subjected the P100 and the corresponding cell lysate to SDS-PAGE and to a subsequent Western blot analysis with an antibody against GFP and the myc-tag (Fig. 23 A). The blots were scanned and the signal intensity was quantified by ImageJ. The ratio between the proteins in the EV fraction und the parent cell lysates was calculated. As shown in the histogram in Fig. 23 B, the release of either GFP-SUMO-2 (white bar) or myc-SUMO-2 (grey bar) within EVs was indistinguishable. 
A

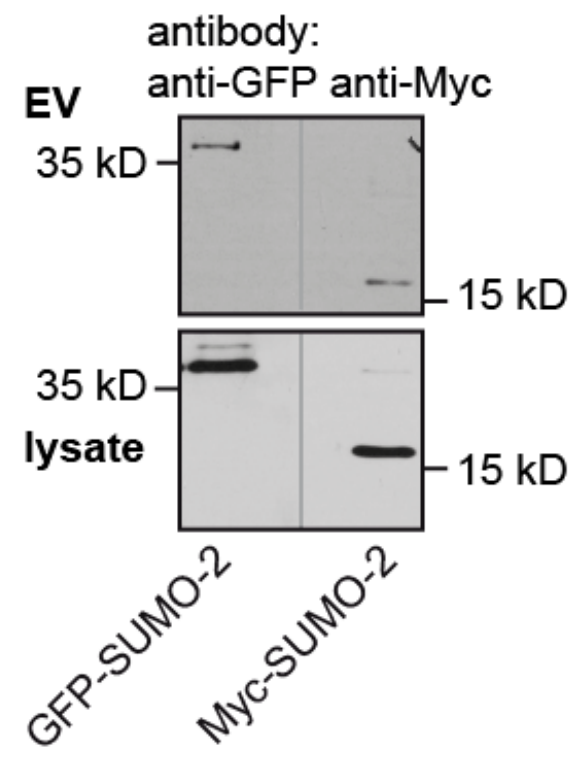

B

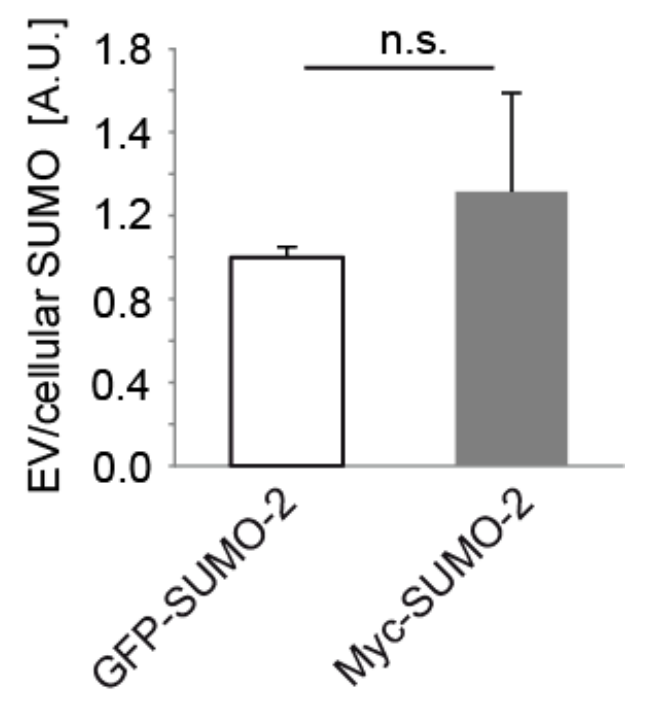

Fig. 23: SUMO-2 fusion leads to extracellular vesicle sorting of GFP

N2a cells were transfected with constructs either encoding for GFP-SUMO-2 $\triangle$ GG or myc-SUMO-2 $\triangle$ GG. (A) EVs and corresponding cell lysates (lys) were analysed by Western blot with antibody against the GFP-tag or the myctag (Please note that the EV and lysate blots were cut for incubation with either anti-GFP or anti-myc antibodies. Exposure times were indentical). Blots were scanned and signal intensities of the bands were quantified. To determine the EV release of both constructs, ratios of SUMO-2 signal intensities in the EV fraction versus cellular fractions were calculated. (B) The histogram (right) displays no significant differences for the EV release of GFPSUMO-2 $\triangle G G$ (white bar), compared to the EV release of a myc-tagged SUMO $\triangle G G$ (grey bar). All values are given as mean + SEM from $n=6$ independent experiments; student's 2-side t-test; n.s. indicates not significant. 


\subsubsection{SUMO-1 also modulates extracellular vesicle sorting of the cytosolic protein GFP}

After demonstrating that SUMO-2 can act as a sorting factor for EV release, we wondered whether SUMO-1 could also mediate sorting to EVs. We transiently transfected N2a cells with either GFP-SUMO-2- $\triangle G G$ or with GFP-SUMO-1- $\triangle G G$ construct. EVs as well as the corresponding cell lysates of transfected cells were processed for Western blot analysis and probed with an antibody against GFP. We found that the GFP-SUMO-1- $\Delta$ GG is sorted to EVs, albeit to a lesser extent as GFP-SUMO-2- $\triangle \mathrm{GG}$ (Fig. $24 \mathrm{~A}$ ).

A

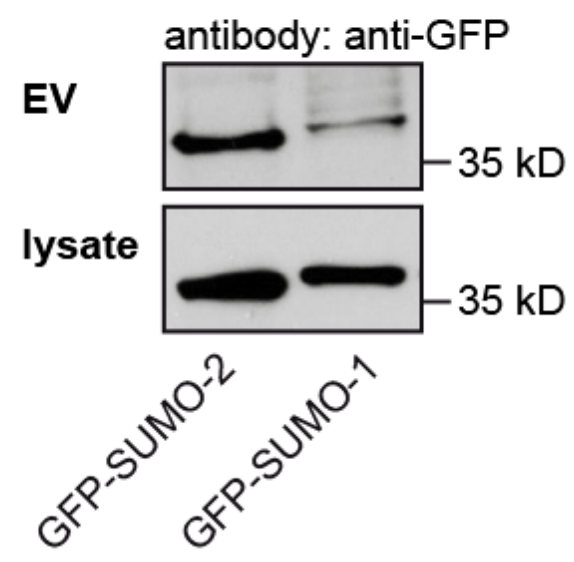

B

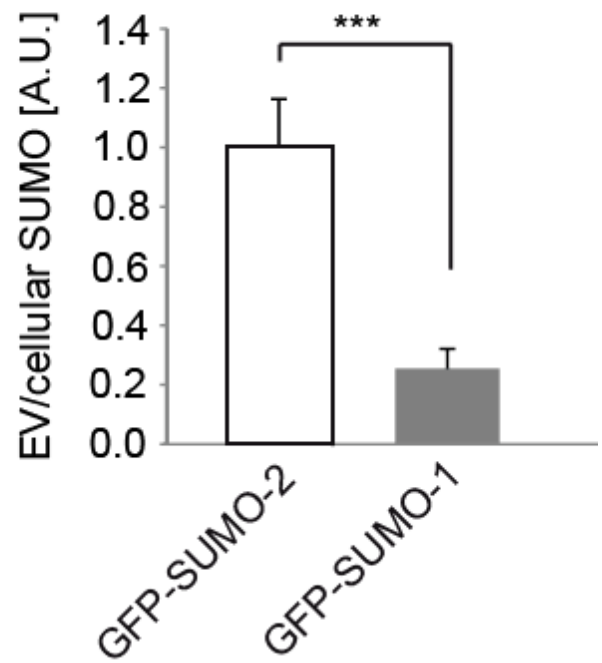

\section{Fig. 24: Comparison of SUMO-1 and SUMO-2 release within extracellular vesicles}

For the determination of EV release, (A) Western blot analysis of EV pellets and parental cell lysates of transfected mouse neuroblastoma cells, with the plasmids indicated above was conducted. (B) For the quantification of EV release, the ratio of GFP signal in the EV fraction versus the cell lysate was calculated. The histogram shows a decrease for GFP-SUMO-1- $\triangle$ GG (grey bar) release within EVs up to 5-fold, compare to the release of GFP-SUMO-2- $\triangle$ GG (white bar). All values are given as mean + SEM of $n=12$ independent experiments; SUMO-2 was arbitrarily normalised to 1 ; student's 2 -side t-test; ${ }^{* \star *}$ indicates $p \leq 0.001$. 


\subsubsection{SUMOylation increases the extracellular vesicle release of the transmembrane protein amyloid precursor protein (APP)}

The amyloid precursor protein (APP) is an integral type I membrane protein .After identifying SUMOylation as a potential sorting factor for the EV release of cytosolic proteins, we wanted to explore whether SUMOylation might also target transmembrane proteins into EVs. Therefore N2a cells were transfected with plasmids either encoding for YFP-APP sw $_{\text {(bearing }}$ the Swedish mutation K670N M671L) or the corresponding C-terminal SUMO-2 fusion

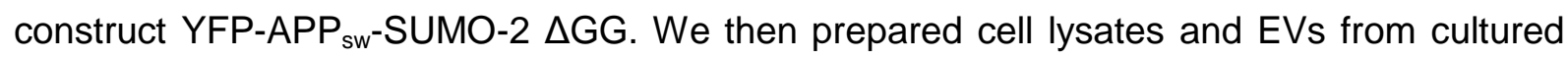
medium, which were subsequently subjected to SDS-PAGE and further subjected to Western blot analysis (Fig. $25 \mathrm{~A}$ ) and probed with an antibody against APP (6E10 see Table 2). To quantify APP secretion with EVs, the ratio of APP in EVs to cell lysates was determined. We found that the EV release of a SUMO fusion protein of YFP-APP $P_{s w}$, is increased compared to YFP-APP $_{\text {sw, }}$ (Fig. 25 B). 
A

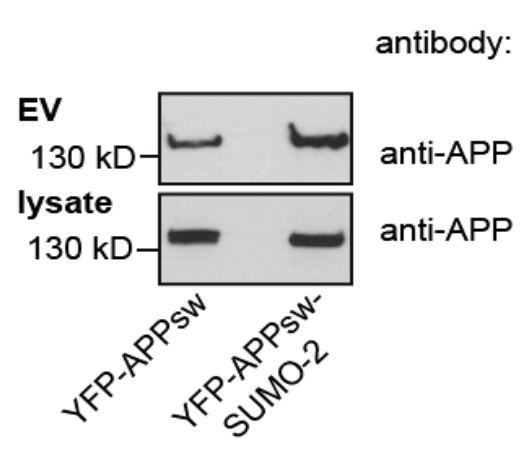

C

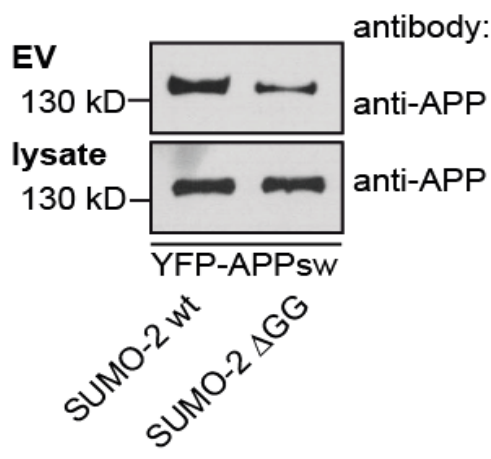

B

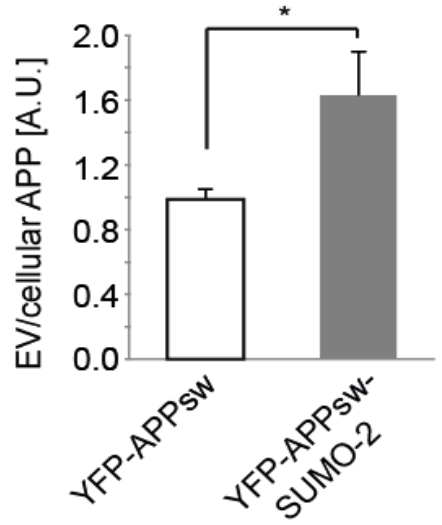

D

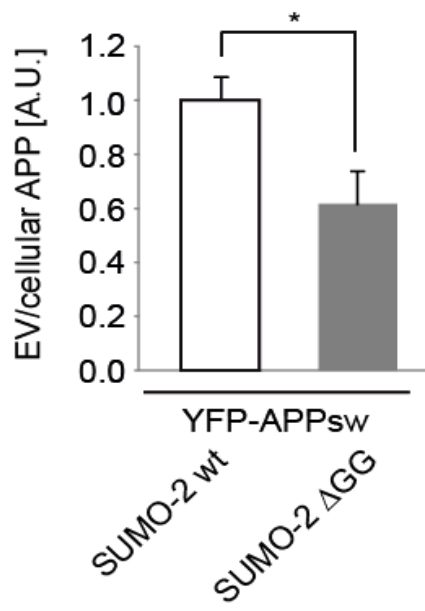

E

sucrose gradient: YFP-APPsw

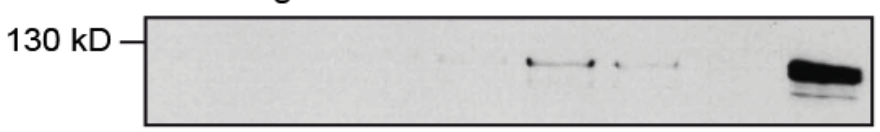

antibody: anti-APP

1.031 .071 .111 .161 .201 .241 .271 .32 lysate sucrose $[\mathrm{g} / \mathrm{ml}]$

sucrose gradient: YFP-APPsw-SUMO-2

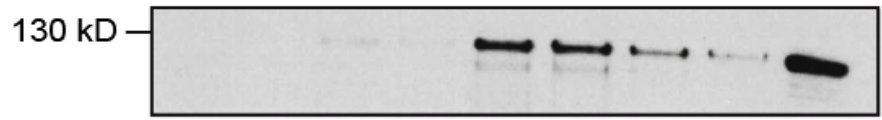

antibody:

anti-APP

1.031 .071 .111 .161 .201 .241 .271 .32 lysate sucrose $[\mathrm{g} / \mathrm{ml}]$

\section{Fig. 25: SUMO-2 increases extracellular vesicle release of the transmembrane protein APP}

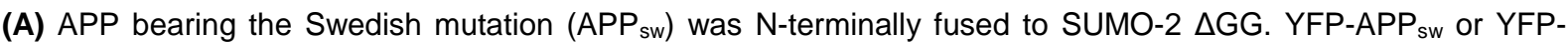

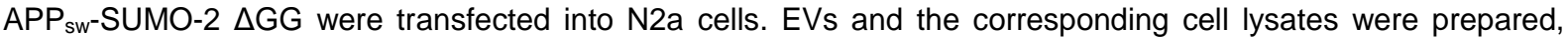
according, to the protocol described previously. Obtained EV pellets and the cell lysates were analysed via Western Blot. (B) Signal intensities of the blots were analysed using Image $\mathrm{J}$ and by calculating the ratio between EV signals versus total cellular APP signals, YFP-APP sw $_{\text {w }}$ was normalised to 1 (histogram upper right panel.). Values are given as mean + SEM from $n=9$ independet experiments; student's 2-side t-test; * indicates $p \leq 0.05$. (C) YFP-APP sw was either co-transfected with wildtype SUMO-2 or with the conjugation deficient mutant SUMO-2 $\triangle G G$. EVs and parental cell lysates were subjected to Western blot analysis. Blots were scanned an analysed for signal intensities. (D) The histogram displays the calculated ratios for EVs versus the total cellular APP (Iys), for SUMO-2 (normalised to 1, white bar) and for the conjugation deficient mutant SUMO-2 $\triangle$ GG (grey bar). Values are given as mean + SEM from $n=9$ independent experiments; student ${ }^{*}$ s 2 -side t-test; ${ }^{*}$ indicates $p \leq 0.05$. 
Likewise, co-transfection of YFP-APP ${ }_{\text {sw }}$ either with SUMO-2 wildtype or with the conjugation deficient mutant SUMO-2- $\triangle G G$ into $\mathrm{N} 2$ a cells resulted in increased release of APP co-transfected with SUMO-2 wt (Fig. $25 \mathrm{D}$ histogram, white bar) as compared to the conjugation deficient mutant SUMO-2- $\triangle \mathrm{GG}$ (Fig. $25 \mathrm{D}$ histogram, grey bar).

Additionally, we used sucrose density gradient to show that APP and APP-SUMO-2 fusion are truly released with EVs. As shown in Fig. $25 \mathrm{E}$ both float at the same density as the EV marker protein Flotillin-2 at 1.20 to $1.27 \mathrm{~g} / \mathrm{mL}$ sucrose. Supporting our quantitative results with ultracentrifugation, a higher proportion of the fusion construct YFP-APP sw $-S U M O-2$ was

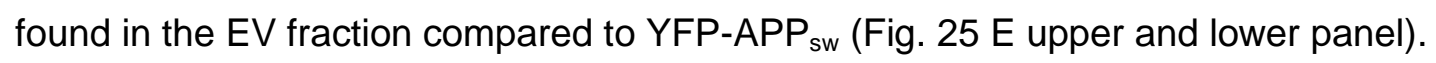

Thus, our data show that SUMO modification not only increases EV release of cytosolic but also at least of one transmembrane protein.

\subsection{Extracellular vesicle release of SUMO-2 is ESCRT-dependent}

We next tried to elucidate the molecular mechanism of SUMO-dependent sorting into EVs. Protein delivery to EVs can be mediated by ESCRT dependent and independent pathways. Therefore, we first blocked components of the ESCRT machinery and assessed SUMO release with EVs under these conditions.

\subsubsection{RNA Interference with the ESCRT components Alix and Tsg101 decrease extracellular vesicle release of a SUMO-2-GFP fusion protein}

To answer the question whether SUMO-2 is targeted to EVs by the ESCRT pathway, we used RNA interference (RNAi) against the ESCRT proteins Tumor susceptibility gene 101 (Tsg 101) and Alix (see Table 4).To test the knockdown efficiency of the used siRNA constructs, cells were treated either with Tsg 101 siRNA, with Alix siRNA or mock treated. After $36 \mathrm{~h}$ incubation time, the cells were lysed with CHAPS buffer as described before. The obtained cell lysates were subjected to Western blot analysis (Fig. 26 A and Fig. 26 C) with antibodies directed against Tsg101, Alix and either Actin or Calnexin as a loading control.

The signal intensities were determined with ImageJ software and the ratios for Tsg 101 to Calnexin and Alix to Actin were calculated. Protein levels of Tsg 101 were down-regulated by approx. $70 \%$ and protein levels of Alix by approx. $90 \%$ (Fig. 26 B and Fig. 26 D). 
A

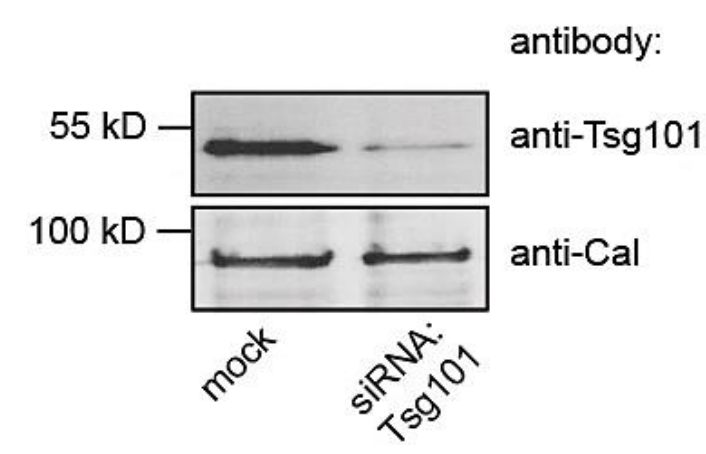

C

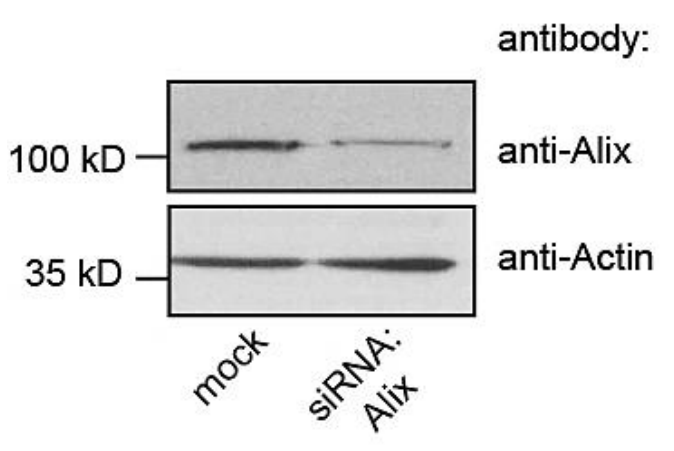

B

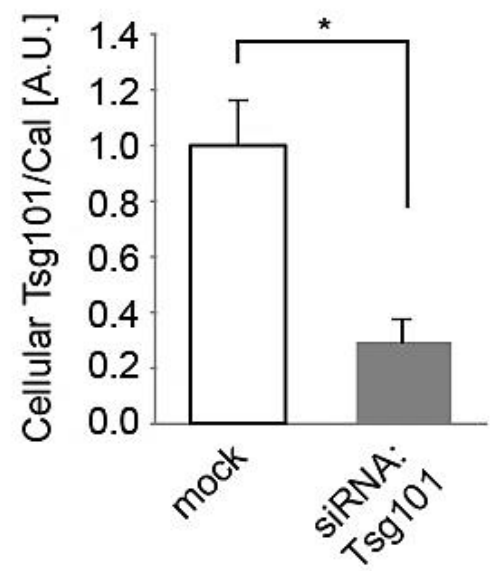

D

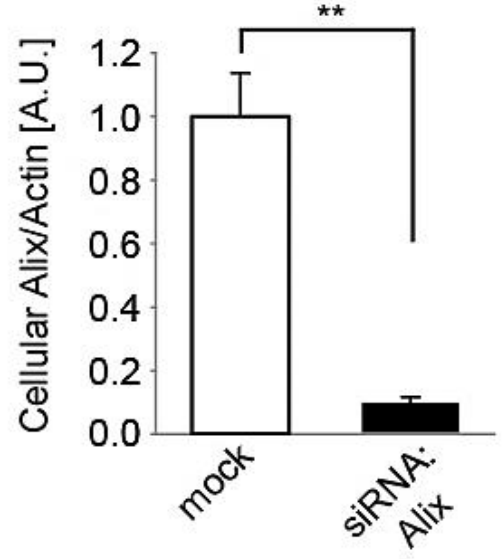

Fig. 26: Down-regulation of Tsg 101 and Alix with siRNA

Efficiency of siRNA-mediated down-regulation was determined by Western blot analysis. (A) Western blot of cell lysates was performed with antibodies against Tsg 101 and Calnexin as loading control. (B) The ratio of signal intensities for Tsg 101 versus Calnexin was calculated for mock (white bar) transfected and siRNA against Tsg 101 treated cells (grey bar). Efficiency of Tsg 101 down-regulation was around $70 \%$. (C) Western blot analysis of mock and Alix siRNA transfected cells with antibodies against Alix and Actin (loading control). (D) The ratio Alix/Actin was quantified for mock treated cells (white bar, normelized to 1 ) and for siRNA transfected cells (grey bar). Efficiency of Alix down-regulation was around $90 \%$. Results are given as mean + SEM from $n=6$ independent experiments for Alix and $n=3$ independent experiments for Tsg101; student's 2-side t-test; * indicates $p \leq 0.05$.

Next, we down-regulated the expression of both ESCRT complex proteins, Alix and Tsg101 and subsequently determined the EV release of a GFP-SUMO-2 fusion protein (see section 2.1.4.2). N2a cells were treated either with siRNA against Alix or with siRNA against Tsg101. As a control cells were also mock treated. After $36 \mathrm{~h}$, the cells were transfected with a construct expressing a GFP-SUMO-2 fusion protein. After $16 \mathrm{~h}$ we harvested the parental cell lysates and prepared EVs from the culture medium. Western blot analysis of lysates and EV fractions revealed RNAi mediated down-regulation of Tsg101 (left panel) or Alix (right panel), resulted in a marked decrease of GFP-SUMO-2 release with EVs. 
A

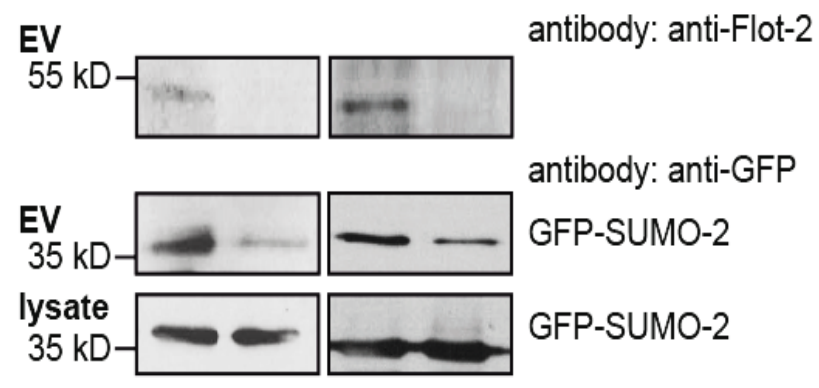

siRNA: Mock Tsg101 Mock Alix
B

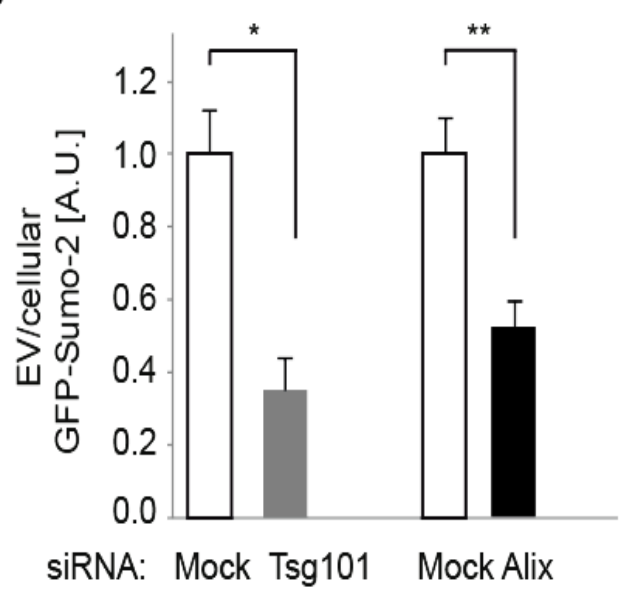

Fig. 27: Alix and TSG101 are required for the extracellular vesicle release of SUMO-2

N2a cells were transfected with siRNAs directed against Alix or Tsg101 and Mock transfected (only treated with oligofectamin). (A) EVs and corresponding cell lysates were prepared and analysed by Western blotting by staining wit antibodies against GFP and Flotilin-2. (B) The histogram shows the calculated ratios for extracellular vesicle GFP signal versus cellular GFP signal in cells treated with siRNA against Tsg101 (grey bar) versus Mock treated cells and for cells treated with siRNA against Alix (black bar) versus Mock treated cells. All results are given as means + SEM for $n=12$ for Alix siRNA and $n=6$ for Tsg101 siRNA experiments. * indicates $p<0.05$ and ${ }^{* *} \mathrm{p}<0.005$; 2-side t-test.

The quantification revealed an approximately 2.8-fold reduction of the EV/cell lysate ratio of GFP-SUMO-2 for Tsg101 RNAi and an approximately 2-fold reduction for Alix siRNA treated $\mathrm{N} 2 \mathrm{a}$ cells (black bar) compared to mock treated controls (Fig. $27 \mathrm{~B}$ ). The responsible protein for the final fission of vesicles is the AAA (ATPase associated in various cellular activities) ATPase VPS4 (vacoular protein sorting 4) (Roxrud et al. 2010). The dominant negative mutation E233Q abrogates the ATP hydrolysis of VPS4 (Bishop et al. 2000) and prevents the budding of vesicles (Roxrud et al. 2010). To elucidate the influence of the dominant negative mutation E233Q on the EV release of SUMO-2, we transiently co-transfected a myc-SUMO2- $\triangle G G$ construct ( $\triangle G G$ mutation prevents the conjugation to SUMO and other proteins) with a plasmid encoding for the dominant negative (dn) mutant of VPS4 E233Q. EVs and the corresponding parental lysates were prepared from conditioned cultured medium and conducted to SDS-PAGE and subsequently conducted to Western blot analysis (Fig. 28 A). We detected a significant decrease in the EV release of a myc-SUMO-2 protein (Fig. 28 B). As an internal control, the WB blot membranes were also probed with an antibody against the EV marker protein Alix (Fig. $28 \mathrm{~A}$ upper panel). Alix release with EVs was decreased upon Vps4dn expression which is consistent with the fact that Alix interacts with the ESCRT machinery. In line with the function of VPS4, expression of its dominant negative form also decreased the total amount of EV release. The number of total released EVs was analysed by nanoparticle tracking analysis in the collecting medium (see Appendix, Table 13). 
A

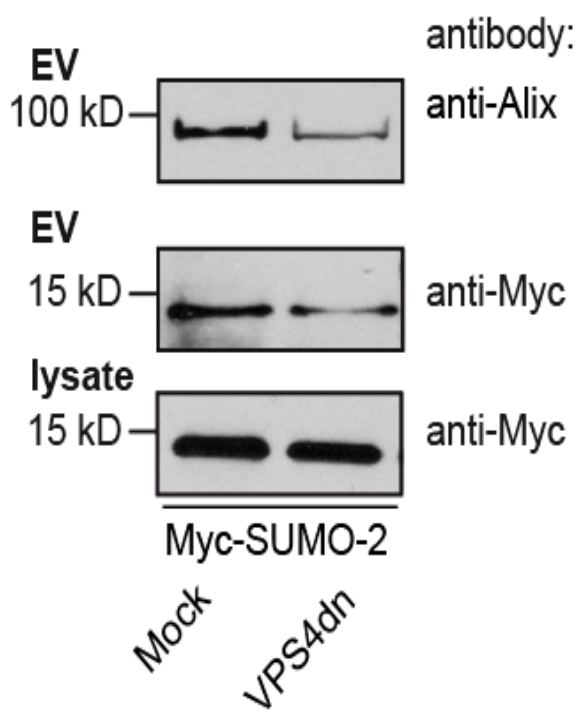

B

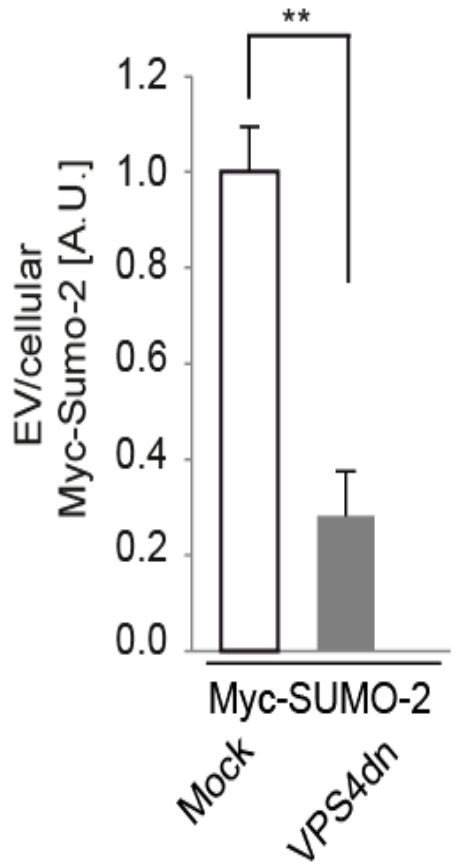

Fig. 28: Release of SUMO-2 with extracellular vesicles is dependent on ESCRT

(A) Myc-SUMO-2 and was transiently co-transfected with the dominant negative VPS4 E233Q mutant and the EV release was determined by Western blot analysis with an antibody against $\alpha$-Syn. The blots of the extracellular vesicle fractions were accessorily probed with an antibody against the EV marker protein Alix. (B) The histogram displays the calculated ratio between signal intensity of the extracellular fraction versus the corresponding cell lysate. All values are given as mean + SEM from $n=4$ independent experiments. ${ }^{* *}$ indicates $p \leq 0.005$; student's 2-side t-test. 


\subsubsection{Co-expression of the dominant negative mutant of VPS4 decreases the extracellular vesicles release of a GFP-SUMO-2 fusion protein}

As positive and negative controls for the effect of VPS4dn overexpression on the release of EVs, we studied the VPS4dn effect on a GFP-SUMO-2 fusion protein, for the Moloney murine leukemia virus Gag protein, fused to a GFP (MLV-Gag-GFP) and for PLP-myc (proteo-lipid protein 1). MLV-Gag is known to be released with EVs in an ESCRT dependent manner (Fang et al. 2007), whereas PLP release with EVs is ESCRT independent and requires ceramide (Trajkovic et al. 2008). As shown in Fig. 29 A-B, VPS4dn co-expression inhibited the EV release of GFP-SUMO-2 and MLV-Gag-GFP compared to mock co-transfection. As expected, there was no change in the EV secretion of PLP-myc, when N2a cells were co-transfected with VPS4dn (Fig. 29 C). Additionally, the membranes were probed with antibodies directed against Tsg101 or Alix as an internal control. As expected, the VPS4dn expression reduced the amount of Tsg101 and Alix in the EV fractions (Fig. 29 A and $\mathrm{B}$ ). For quantification, the blots were scanned and quantified for their signal intensities to calculate the ratio of EV versus cellular protein. This quantification revealed a significant reduction of the EV release of MLV-Gag-GFP (grey bar) and GFP-SUMO-2 (black bar), due to the inference with VPS4dn E233Q (Fig. 29 D). Taken together, the EV release of SUMO-2 requires several components of the ESCRT machinery. 
A

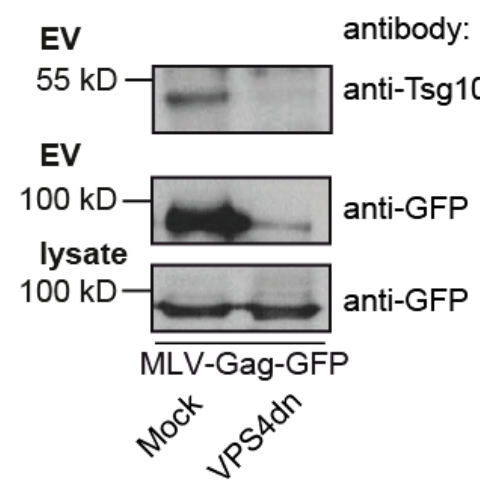

B

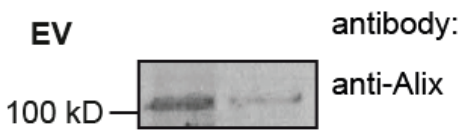

EV

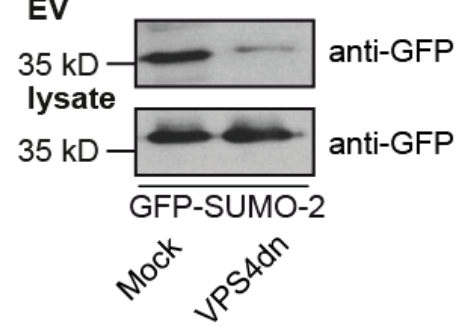

C

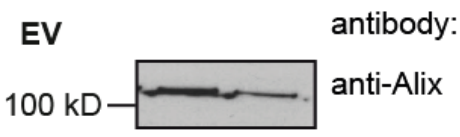

EV

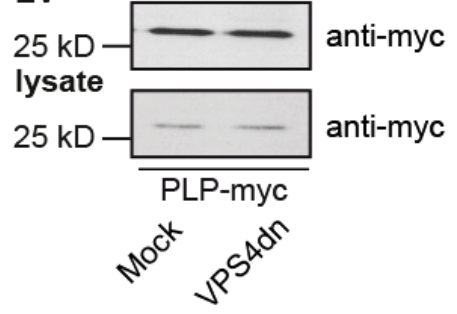

D

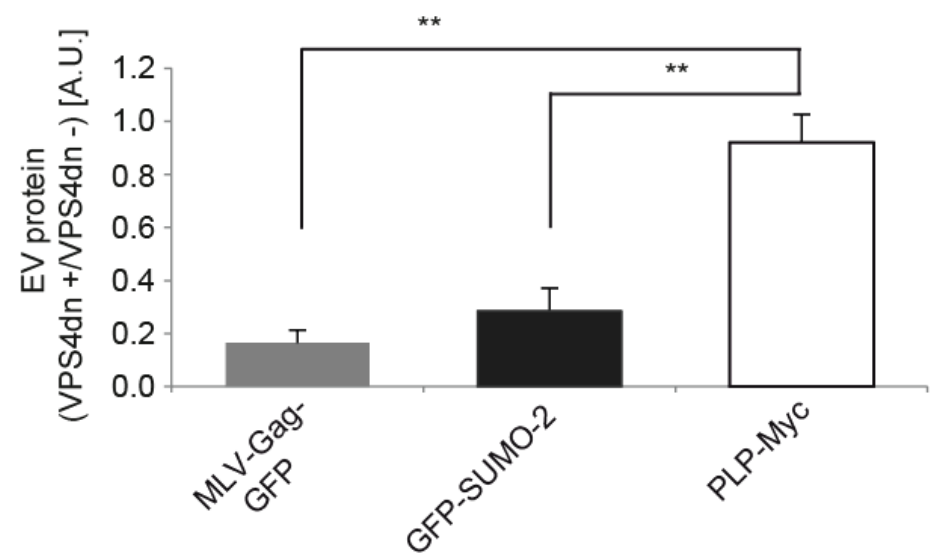

Fig. 29: Interference with VPS4 function inhibits release of SUMO-2 within extracellular vesicles

(A-C) N2a cells were co-transfected with VPS4dn and either MLV-Gag-GFP or GFP-SUMO-2 $\triangle$ GG or PLP-Myc. Cells transfected with MLV-Gag-GFP, GFP-SUMO-2 $\triangle$ GG or PLP-Myc alone were used as controls. EVs were prepared and cell lysates and vesicle pellets were subjected to Western blotting and probed with anti-GFP and anti-Myc antibodies. Blot membranes were also re-probed with antibodies against the EV marker proteins Tsg101 and Alix (AIP1). (D) The ratio of protein in the EV fraction from cells co-transfected with VPS4dn to mocktransfected cells was quantified by analysing signal intensities on the Western blots via ImageJ software. The negative control PLP-myc was normalised to 1 . All values are given as mean + SEM for $n=5$ independent experiments, ${ }^{* *}$ indicates $\mathrm{p}<0.005,2$-side t-test. 


\subsubsection{Extracellular vesicle release of SUMO-2 does not depend on the canonical SUMO protein interaction motif Q30 F31 I33}

We next asked how SUMO-2 would interact with the ESRCT machinery. Tsg101 contains a SUMO-interaction motif for non-covalent interaction with SUMO proteins. We therefore assumed that SUMO interaction with the ESCRT could be mediated by protein-protein binding. Mutation of a canonical protein interaction motif Q30, F31, I33 in SUMO-2 had recently been shown to disrupt binding to SUMO interacting proteins (Hecker et al. 2006, Sun et al. 2007). We therefore compared EV release of SUMO-2 wt-GFP and the Q30A F31A I33A triple mutant of SUMO-2 GFP (SUMO-2- $\Delta$ SIM). For further analysis we subjected cell lysates and EV fractions to SDS-PAGE and subsequently to Western blot analysis. The membranes were probed with an antibody against GFP (Fig. $30 \mathrm{~A}$ ). The ratio of EV/cellular SUMO-2GFP was increased for SUMO-2 bearing the $\triangle$ SIM mutant as compared to wt SUMO-2 GFP (Fig. 30 B).

A

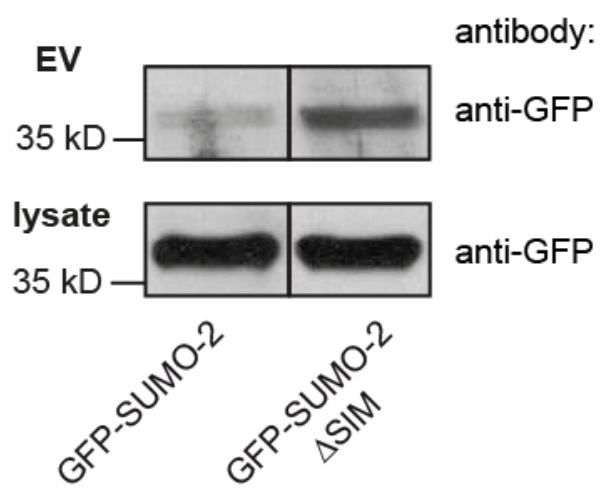

B

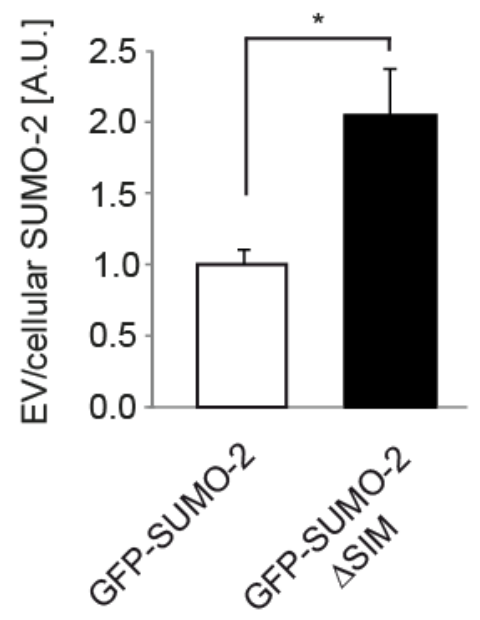

Fig. 30: Mutation of the SIM motif in SUMO-2 increases extracellular vesicle release of SUMO-2

(A) Western blotting of EV pellets and cell lysates from N2a cells transfected with either GFP-SUMO-2 or the triple A mutant (GFP-SUMO-2 $\triangle$ SIM). The blots were scanned and analysed for protein signal intensites. (B) The histogram depicted the ratio of GFP-SUMO-2 (white bar) versus the $\triangle$ SIM mutant (black bar) signal intensities of EV pellets versus the corresponding cell lysates. GFP-SUMO-2 was normalized to 1. All values are given as means + SEM for $n=10$ independent experiments, * indicates $p<0.05$, 2-side t-test.

Based on this observation, we assumed that the release of GFP-SUMO-2 within EVs is not mediated by a classical SIM protein-protein interaction. A possible explanation for this finding is that mutations of the SIM interaction motif might increases the amount of unbound cytosolic SUMO-2, which would then be available for EV release. 


\subsection{SUMO-lipid interaction}

\subsubsection{SUMO-2 interacts with phosphoinositols}

The previous section 3.5.3 has shown, that EV sorting of SUMO-2 is not promoted via SIMmediated protein interaction. We therefore investigated, whether the EV sorting and release of SUMO-2 was driven by an interaction with lipids at the ESCRT formation site. It has been shown that for a variety of SUMO-2 interacting proteins, like the EV protein polymyositisscleoderma overlap syndrome (PMSCL1), the tumor suppressor protein promyelocytic leukaemia (PML) and the E3 SUMO ligase protein inhibitor of activated STAT 1 (PIAS1), phosphorylation of serine residues in the SUMO interaction domain is required for SUMO binding. This suggests an interaction of SUMO-2 with negatively charged domains (Stehmeier et al. 2009).

We therefore established an assay to test for SUMO-2 binding to different, negatively charged lipids. For this assay, recombinant SUMO-2 was labelled with the polarity-sensitive excited state intramolecular proton transfer (ESIPT) probe MFM (Shvadchak et al. 2011) and purified as described in section 2.2.4.5. Small unilamellar vesicles (SUV) were prepared from mixtures of 1-palmitoyl,2-oleoyl-sn-glycero-3-phosphocholine (POPC) in various combinations with different negatively charged lipids: phosphatidylserine (POPS, 10\%) and a low fraction (5\%) of the phosphoinositides $\mathrm{PI}(3) \mathrm{P}, \mathrm{PI}(5) \mathrm{P}, \mathrm{PI}(3,5) \mathrm{P}_{2}, \mathrm{PI}(4,5) \mathrm{P}_{2}$ or $\mathrm{PI}(3,4,5) \mathrm{P}_{3}$. Briefly, a stock solution of labelled SUMO-2-MFM was diluted to $100 \mathrm{nM}, 200 \mathrm{nM}$ and $300 \mathrm{nM}$; the dilutions were mixed with different SUV concentrations of each lipid indicated above (up to $120 \mu \mathrm{M}$ ) and transferred into a 96 well quartz glass microplate. To equilibrate the whole system an incubation time of at least $10 \mathrm{~min}$ was chosen. Subsequently the fluorescence of the MFM probe was recorded in a plate reader and the data were analysed with a tool implemented in the program Mathematica (Wolfram Research).

As shown in Fig. 31 the individual affinity of each lipid for SUMO-2 was calculated from a global analysis of the obtained and combined data of the fluorescence recording (all data analysis for this approach was kindly performed by Thomas M. Jovin, Laboratory of Cellular Dynamics, MPI for Biophysical Chemistry, Göttingen). 


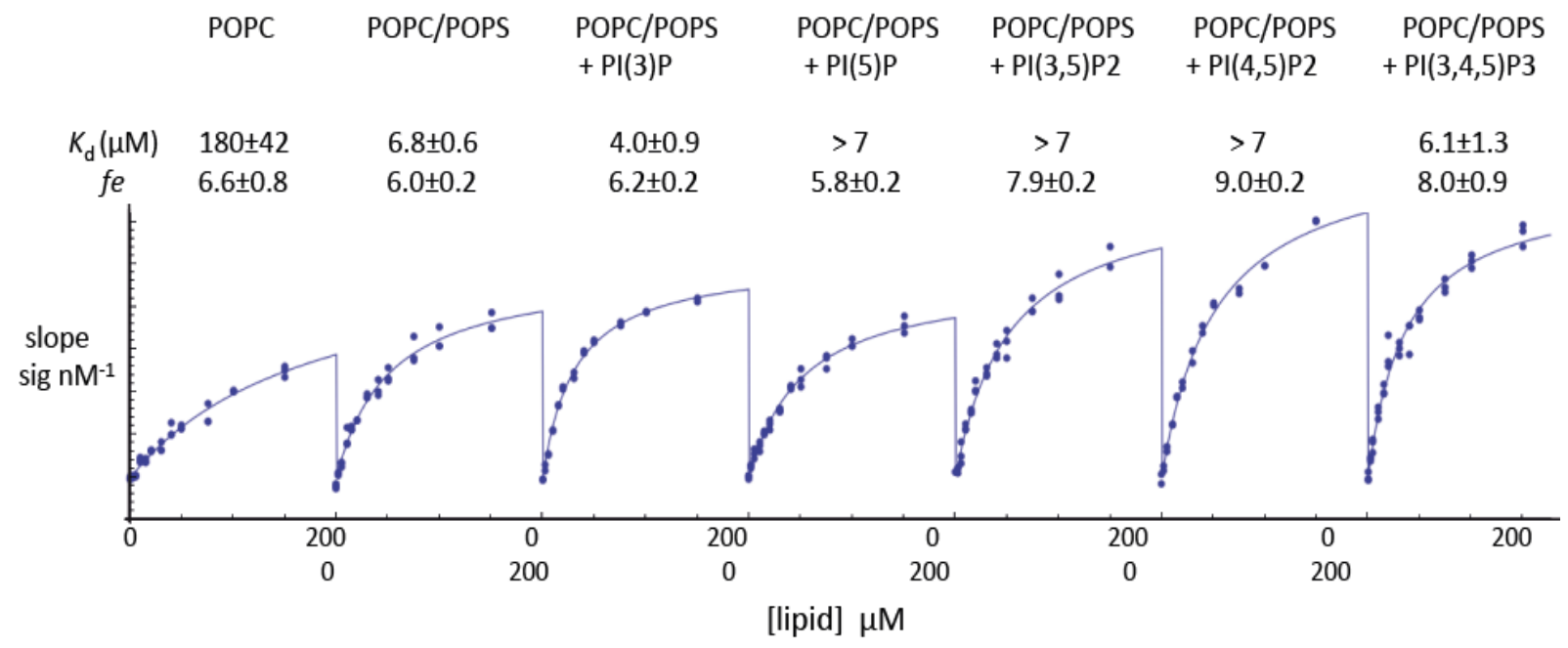

Fig. 31: Microplate titration assay of SUMO-2-MFM

The binding affinities of each lipid that contributes to the apparent affinity of the protein for the liposome was calculated as described in the Methods part. $K_{d} S$ are given \pm standard measurement errors. The values corresponding to $\mathrm{PI}(5) \mathrm{P}, \mathrm{PI}(3,5) \mathrm{P}_{2}$ and $\mathrm{PI}(4,5) \mathrm{P}_{2}$ were too high to be determined (affinity less than that of the POPS co-lipid, i.e. $>7 \mu \mathrm{M})$. Statistically significant differences were obtained for POPC versus POPS $(p<0.001)$, and POPS versus $\mathrm{PI}(3) \mathrm{P}(\mathrm{p}=0.01)$. No significant difference is found for $\mathrm{PI}(3,4,5) \mathrm{P}_{3}$ versus POPS; $n=19$ titrations for each lipid mixture. The fluorescence enhancement factors $(f e)$ are indicated with their respective standard measurement errors. See Methods for further details of this novel measurement approach.

As indicated in Fig. 31, SUMO-2 binds with weak affinity to uncharged membranes $\left(\mathrm{K}_{d}\right.$ for DOPC binding: $180 \mu \mathrm{M}$ ) and with moderate affinity to a variety of PIPs. Highest affinities were observed for $\mathrm{PI}(3) \mathrm{P}$ with a $\mathrm{K}_{d}$ of $4 \mu \mathrm{M}$ and for $\mathrm{P}(3,4,5) \mathrm{P}_{3}$ with a $\mathrm{K}_{d}$ of $6.1 \mu \mathrm{M}$, indicating a preferential binding to $\mathrm{PI} 3 \mathrm{P}$ and $\mathrm{PI}(3,4,5) \mathrm{P}_{3}$. 


\subsection{Identification of the membrane interaction motif of SUMO-2}

\subsubsection{The membrane interaction motif of SUMO-2 is localised to the hydrophobic cleft and nearby loops}

To map the membrane interaction motif in SUMO-2 we collaborated with the group of Prof. M. Zweckstetter, DZNE Göttingen. Germany. For this purpose, recombinant SUMO-2 was expressed and purified as described in section 2.2.1.6 and the NMR analysis was performed according to the protocol presented in section 2.2.6. To identify the membrane interaction

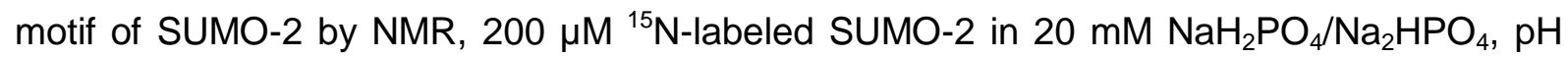
$6.8,100 \mathrm{mM} \mathrm{KCl}, 1 \mathrm{mM}$ DTT was titrated with increasing concentrations of 8,16 and $32 \mathrm{mM}$ DHPC (1,2-dihexanoyl-sn-glycero-3-phosphocholine).

By NMR the major residues which might mediate interaction with lipid membranes were mapped to the hydrophobic cleft of SUMO-2 between the second $\beta$-strand and the $\alpha$-helix (F31, K32, I33, L42 and Y46) Additional residues were located to the loops at the $\mathrm{N}$-terminus of SUMO-2 (H16, H36 and D62) (Fig. 32). To sum up these results, we assume that the main membrane interaction motif of SUMO-2 is localised at the N-terminal end of the hydrophobic cleft and nearby loops. 
A

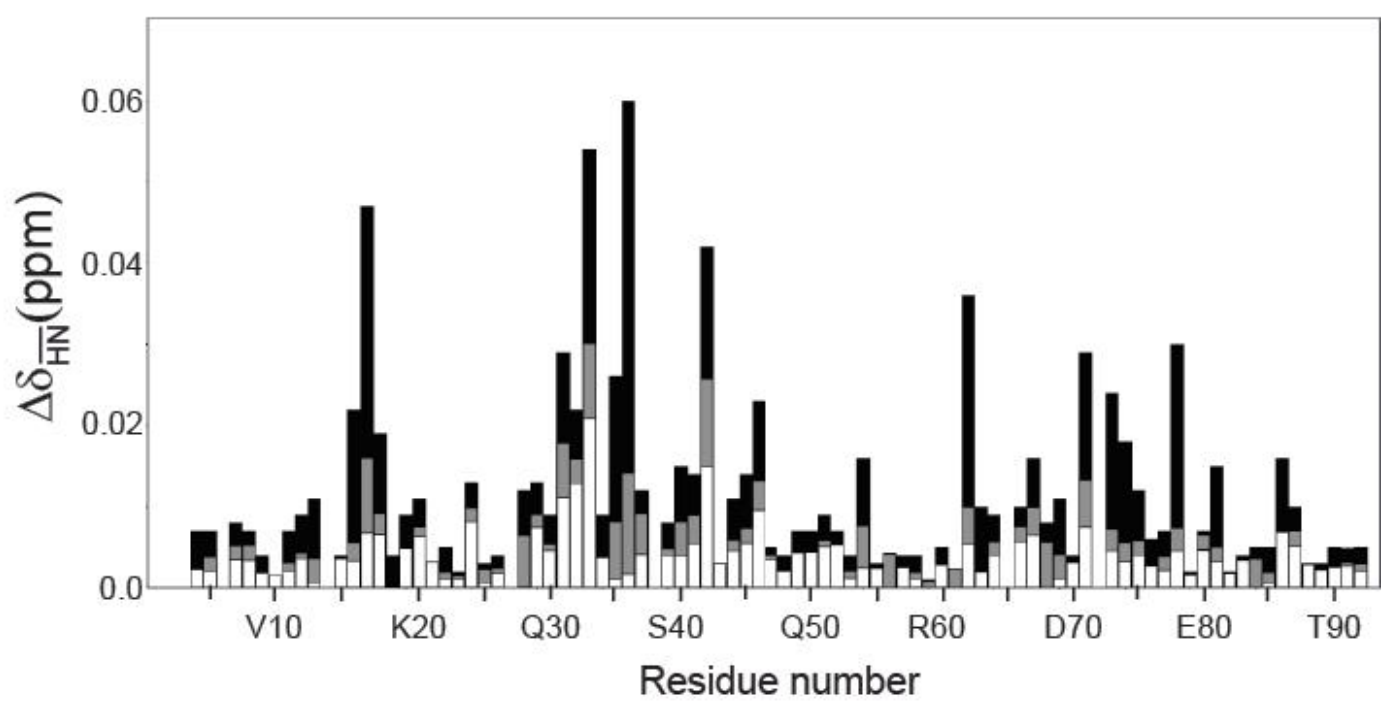

B

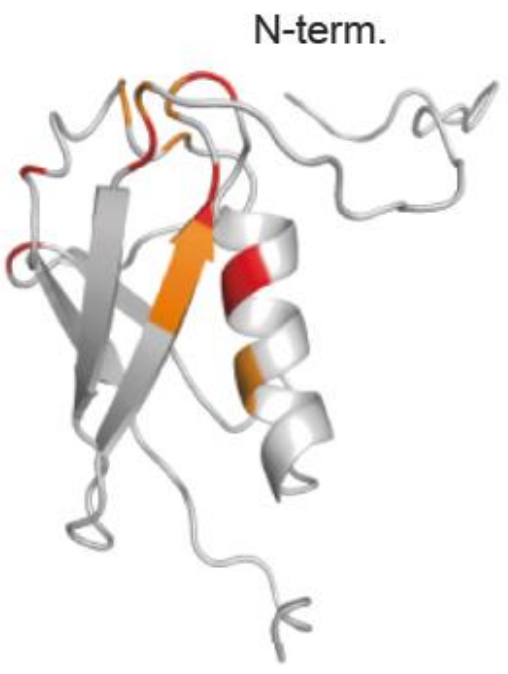

C-term.
C

C-term.

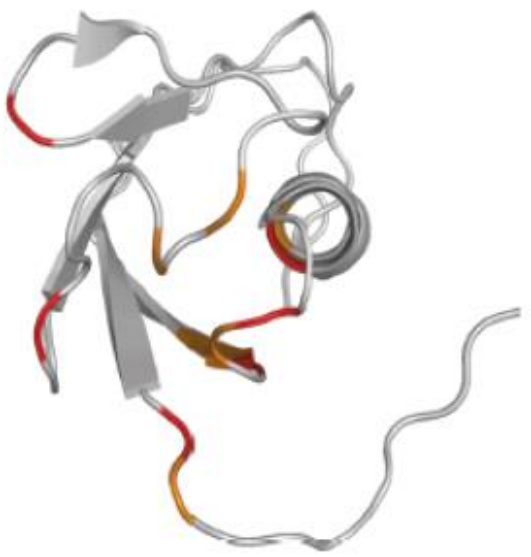

N-term.

Fig. 32: Membrane binding of SUMO-2 analysed by NMR spectroscopy

(A) Mean weighted ${ }^{1} \mathrm{H}^{15}{ }^{15} \mathrm{~N}$ chemical shifts of Sumo-2 at DHPC concentrations of $8 \mathrm{mM}$ (white bars), $16 \mathrm{mM}$ (grey bars) and $32 \mathrm{mM}$ (black bars). Below the critical micellar concentration (CMC) of DHPC of $16 \mathrm{mM}$ only few chemical shift changes in SUMO-2 were observed. $(\mathbf{B}+\mathbf{C})$ The chemical shift perturbation at $32 \mathrm{mM}$ DHPC is plotted onto the SUMO-2 NMR structure (pdb-code: 2AWT). Residues in red display a perturbation greater than $0.03 \mathrm{ppm}$ and residues in orange between $0.02-0.03 \mathrm{ppm}$. The structure in panel (C) is rotated by $90^{\circ}$ relative to panel. 


\subsubsection{Mutations in the hydrophobic cleft and N-terminally loop domains of SUMO-2} decreases the membrane binding propensity of SUMO-2

To prove that the membrane interaction motif of SUMO-2 is located to the hydrophobic cleft and the $\mathrm{N}$-terminally loop, additional experiments were performed to study how a mutation in the residues would affect the recruitment to membranes and the sorting of mutant SUMO into the EVs. Therefore, conjugation-deficient SUMO-2 $\triangle G G$ cDNA with BamH and Xhol restriction sites was synthesized containing the mutations Q30A, F31A, K32A, I33A, L42A, and Y46A ("cleft mutant") and with the mutations H16A, Q30A, F31A, K32A, I33A, H36A, L42A, Y46A, and D62A ("cleft and loop mutant"). The cDNA was cloned into pcDNA 3 Myc vector via $B a m H l$ and $X h o l$ restriction sites. To determine membrane binding of these mutants, N2a cells were transiently transfected either with myc-SUMO-2- $\Delta$ GG cleft, mycSUMO-2- $\Delta$ GG cleft+loop or myc-SUMO-2- $\Delta$ GG. To separate membranes and the cytosolic supernatant, cells were washed with PBS and collected in homogenization buffer. Subsequently, the cells were mechanically disrupted and centrifuged, to remove nuclei and cell debris. The obtained postnuclear supernatant was subjected to ultracentrifugation to separate the membrane and cytosol containing fractions. The membrane pellet and the corresponding cytosolic fractions were subjected to SDS-PAGE and Western blot analysis. As indicated in Fig. 33, we found that both, the myc-SUMO-2-cleft and the myc-SUMO-2 cleft+loop mutant, significantly decrease the membrane binding propensity of SUMO-2 compared to the myc-SUMO-2- $\triangle \mathrm{GG}$ construct (Fig. $33 \mathrm{~A}+\mathrm{B}$ ). 
A

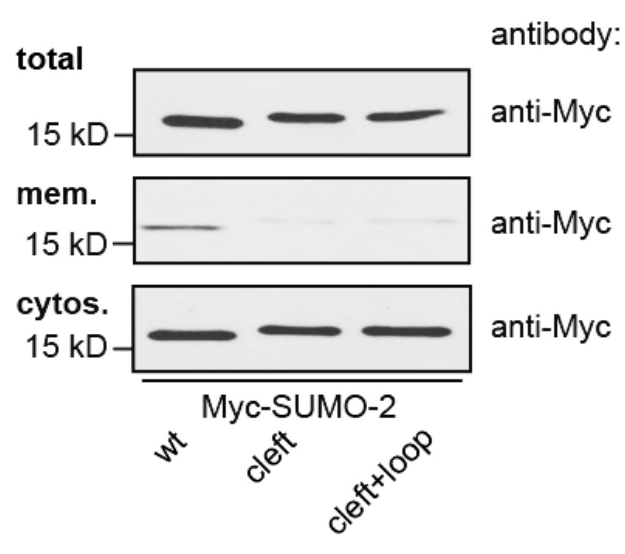

C

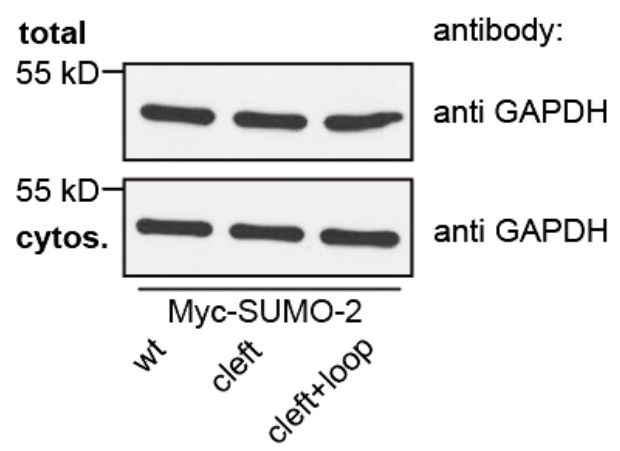

B
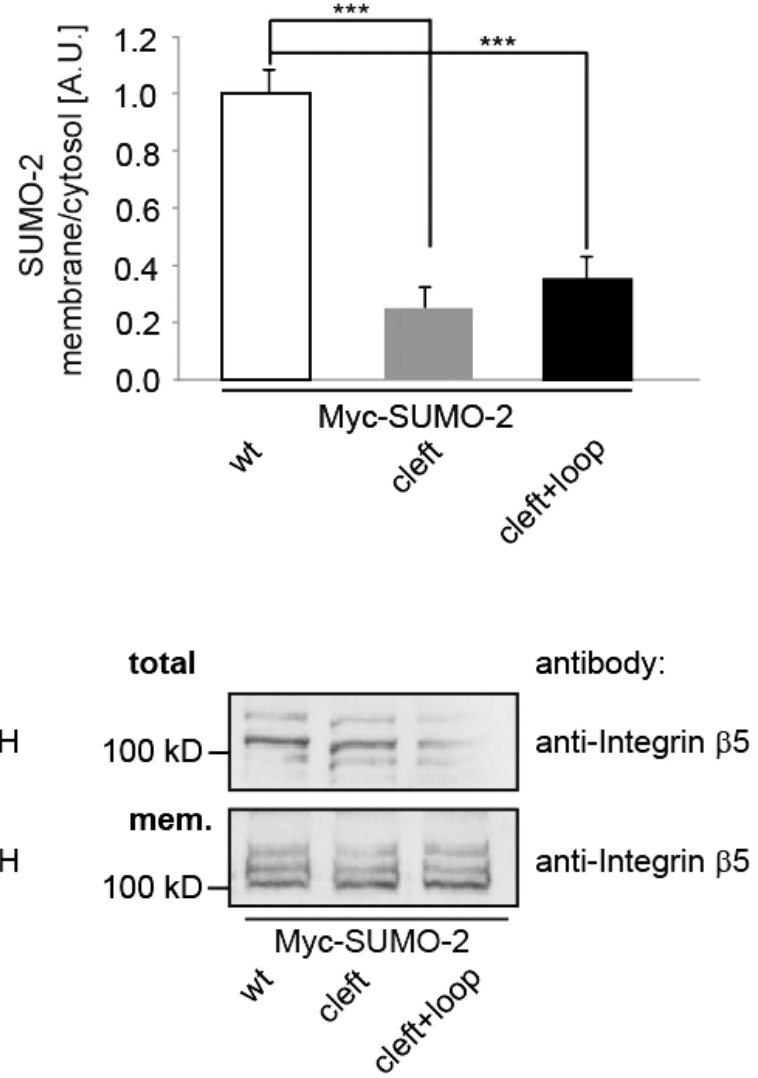

Fig. 33: Mutation of amino acids H16A, Q30A, F31A, K32A, I33A, H36, L42A, Y46A and D62 in the cleft and loop domains of SUMO-2 decreases membrane binding

(A) N2a cells were transfected with Myc-SUMO-2, Myc-SUMO-cleft mutant or Myc-SUMO-cleft+loop mutant. The postnuclear supernatant of the mechanically disrupted cells was centrifuged at $196,000 \times g$ for 30 min to separate the membrane containing pellet and the cytosolic supernatant. Membrane pellets and a proportion of the total cell lysate and the cytosol-containing supernatant were subjected to Western blot analysis and immunostained with an anti-myc antibody. (B) The Histogram displays the quantification of the Western blot analysis by calculating the ratio of signal intensities for membrane versus cytosolic fractions for wt (white bar, normalised to 1), for the cleft mutant (grey bar) and for the cleft+loop mutant (black bar). All values are given as means+SEM for $n=8$ independent experiments, ${ }^{* *}$ indicates $\mathrm{p}<0.0005,2$-side $\mathrm{t}$-test. (C) As fraction controls, blots were re-probed with an antibody against GAPDH as a cytosolic marker and with an antibody against $\beta 5$-Integrin, as a membrane marker. 


\subsubsection{Mutations in the hydrophobic cleft and N-terminally loop domains of SUMO-2} decreases sorting into extracellular vesicles

If SUMO-2 interaction with the ESCRT pathway requires lipid binding, we would expect that mutations which interfere with SUMO-2 lipid binding would inhibit SUMO-2 release with EVs. N2a cells were transfected with SUMO-2- $\Delta$ GG cleft, SUMO-2- $\Delta G$ G cleft+loop mutants and SUMO-2- $\triangle G G$ and quantified EV release. After $8 \mathrm{~h}$ post-transfection we changed the medium and collected EVs for 16 hours. EVs and the corresponding cell lysates were subjected to Western blot analysis (Fig. $34 \mathrm{~A}$ ). By determining the signal intensities of the Western blots and by calculating the ratio of SUMO-2 in EV versus cell lysates we found that both mutants were significantly decreased in the EV fractions (Fig. $34 \mathrm{~B}$, white bar).

A

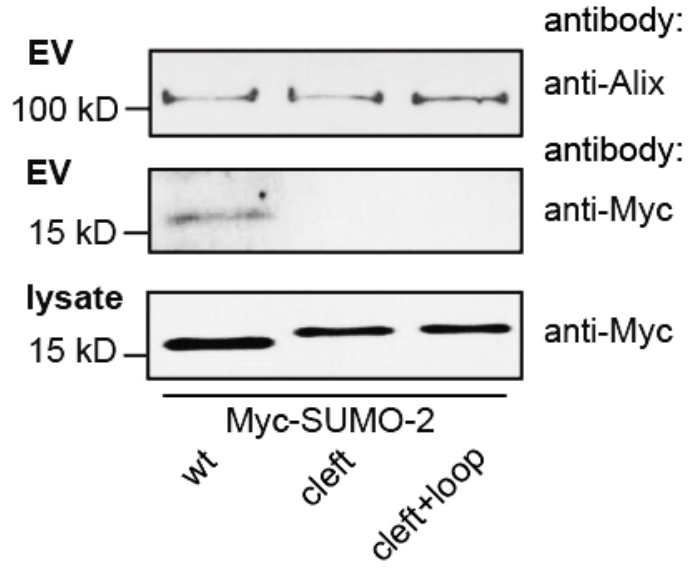

B

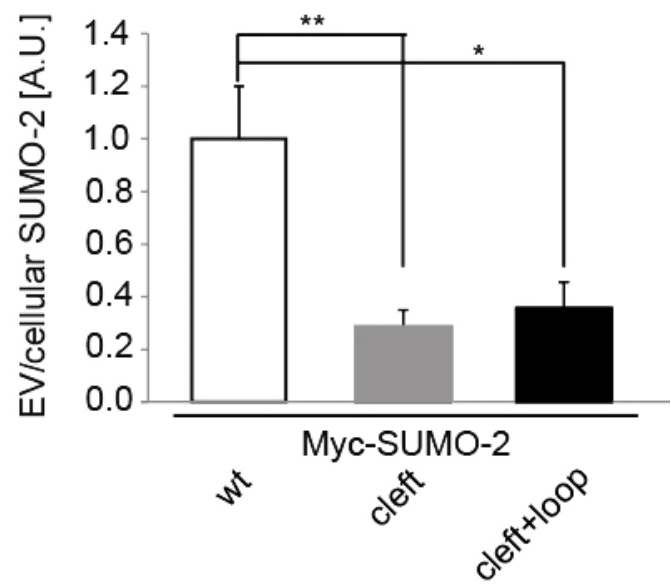

Fig. 34: Mutated amino acids H16A, Q30A, F31A, K32A, I33A, H36, L42A, Y46A and D62 in the cleft and loop domains of SUMO-2 decreases extracellular vesicle sorting

(A) Extracellular vesicles and corresponding lysates were prepared of cells, transfected either with Myc-SUMO-2cleft mutant or Myc-SUMO-2-cleft+loop mutant and with Myc-SUMO-2 serving as a control. Lysates and EV fractions were processed to Western blotting and probed with an antibody against the myc-tag. Membranes were re-probed with an antibody against Alix as a marker for EVs in the different preparations. (B) Blots were scanned and analysed with Image $\mathrm{J}$ software to determine the signal intensities. Thereafter the ratio between extracellular protein versus cellular protein was calculated. All values are given as mean+SEM of $n=9$ independent experiments, ${ }^{*}$ indicates $p<0.05,{ }^{* *}$ indicates $p<0.005,2$-side t-test.

To conclude this, we can assume that the membrane interaction motif of SUMO-2 is located to the hydrophobic cleft and the N-terminally loop of SUMO-2, due to the decreased membrane binding and release within EVs of both SUMO mutant versions. 


\subsection{Co-expression of the dominant negative mutant of VPS4 decreases extracellular vesicle release of $\alpha$-Synuclein}

In line with the observations in section 3.5.1 (Fig. 28) we wanted to elucidate the influence of the dominant negative ( $\mathrm{dn}$ ) mutant of VPS4 E233Q on the EV release of myc- $\alpha-S y n-S U M O-2$ fusion construct. To determine the EV release, a myc-a-Syn-SUMO-2 fusion construct (bearing the $\triangle G G$ mutation to prevent the conjugation to SUMO and other proteins) was cotransfected with a plasmid encoding for a dominant negative (dn) mutant of VPS4 E233Q. EVs and the corresponding parental lysates were prepared from conditioned cultured medium and conducted to SDS-PAGE and subsequently analysed by Western blotting (Fig. $35 \mathrm{~A})$. We detected a significant decrease in the EV release of the myc- $\alpha$-Syn-SUMO-2 fusion protein when co-expressed with the mutant Version of VPS4dn (Fig. 35 B).

A

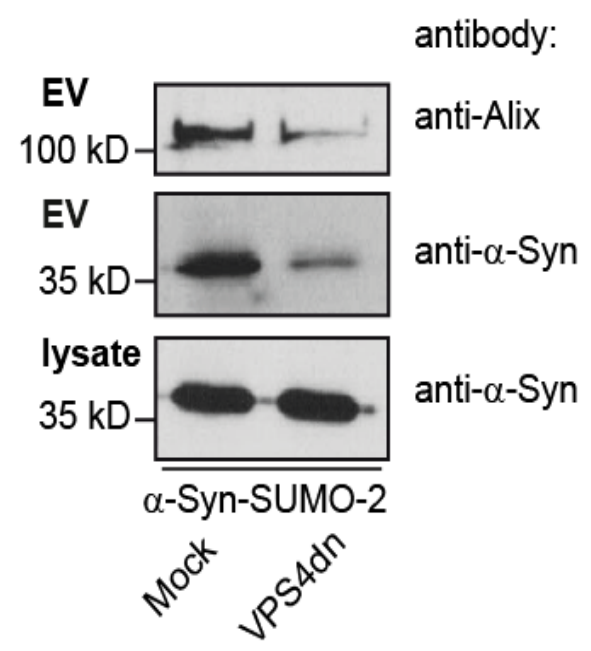

B

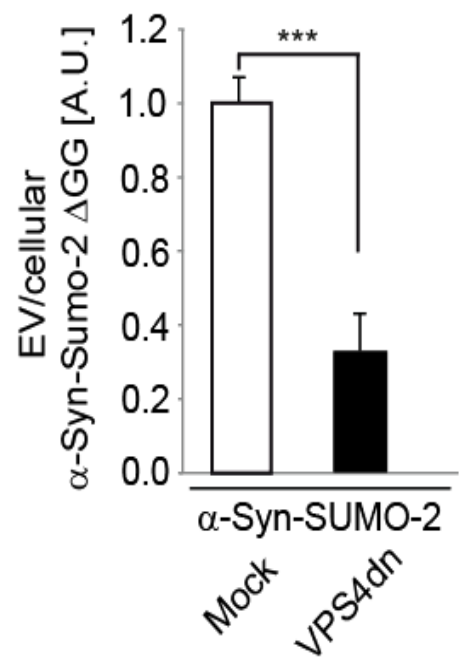

Fig. 35: Release of $\alpha$-Syn-SUMO-2 fusion protein with extracellular vesicles is dependent on the ESCRT

(A) Myc- $\alpha$-Syn-SUMO-2 were transiently co-transfected with the dominant negative VPS4 E233Q mutant and the EV release of both constructs was determined by Western blot analysis with antibodies against $\alpha$-Syn and myctagged SUMO-2. The blots of the extracellular vesicle fractions were subsequently probed with an antibody against the EV marker protein Alix. (B) Quantification of protein signal intensity analysis was performed via ImageJ software. The histogram indicates the calculated ratio of sumoylated extracellular vesicle $\alpha$-Syn versus the parental cell lysate of single transfected N2a cells (Mock, white bar), compare to cells which were cotransfected with the mutated version of VPS4. All values are given as mean + SEM of $n=12$ independent experiments. ${ }^{* *}$ indicates $\mathrm{p}<0.0005 ; 2$-side t-test.

By nanoparticle tracking analysis of the cultured medium (see section 2.2.3.3) we could observe a significant reduction of EV release in the case of VPS4dn co-transfection (see Appendix, Table 13). To conclude this, similar to SUMO-2, the release of $\alpha$-Syn within EVs was inhibited by co-expression of a dominant negative VPS4 mutant, indicating that EV sorting by SUMO modification is ESCRT-dependent. 


\subsection{Inhibition of endosome maturation by overexpression of dominant negative Rab5 does not trap a-Syn or SUMO-2 in enlarged intraluminal vesicles}

Next we wanted to differentiate between the release of vesicles promoted by plasma membrane shedding and release which is mediated by the MVB pathway. To address this issue we co-expressed constructs encoding for myc- $\alpha$-Syn wt or myc-SUMO-2 wt, together with the dominant negative mutant of Rab5 (Rab5Q79L).

Rab5Q79L induces homotypic fusion of early endosomes and stops the endosomal maturation at the level of early endosomes by inhibiting the intraendosomal trafficking (Stenmark et al. 1994a, Stenmark et al. 1994b, Raiborg et al. 2001). This leads to the trapping of ILV like structures within enlarged endosomes and allows the visualisation of proteins sorted into ILVs by immune fluorescence (Trajkovic 2008, Baietti 2012).

Previously, it was shown by electron microscopy that the giant early endosomes induced by Rab5Q79L overexpression are filled with intraluminal vesicle (Trajkovic et al. 2008). Their morphology does not differ from the ILVs detected in MVBs (Trajkovic et al. 2008). The authors also showed by immunofluorescence analysis that EV marker proteins such as Flotillin-2 were trapped in the intraluminal vesicles of Rab5Q79L positive endosomes. We cotransfected N2a cells either with myc-SUMO-2- $\Delta G G$ or with myc- $\alpha-S y n-S U M O-2-\Delta G G$ and rab5Q79L and performed an immunostaining after 24 hours with an antibody against the myc-epitope of SUMO-2, PLP and with an antibody against $\alpha$-Syn.

As a positive control, we performed the same experiment with co-expression of rab5Q79L and PLP-myc which has recently been shown to accumulate in ILVs of rab5Q79L positive endosomes (Trajkovic 2008). As indicated in Fig. 36 A and B (left panel red arrows) we found PLP in rab5Q79L induced endosomal ILVs. In contrast, SUMO-2 (Fig. $36 \mathrm{~A}$, right panel) and a-Syn (Fig. $36 \mathrm{~B}$, right panel) were absent from enlarged rab5Q79L positive endosomes. This data indicates that SUMO-2 and a-Syn may not be sorted via the endosomal MVB pathway but rather bud from the plasma membrane within shedding microvesicles. 
A
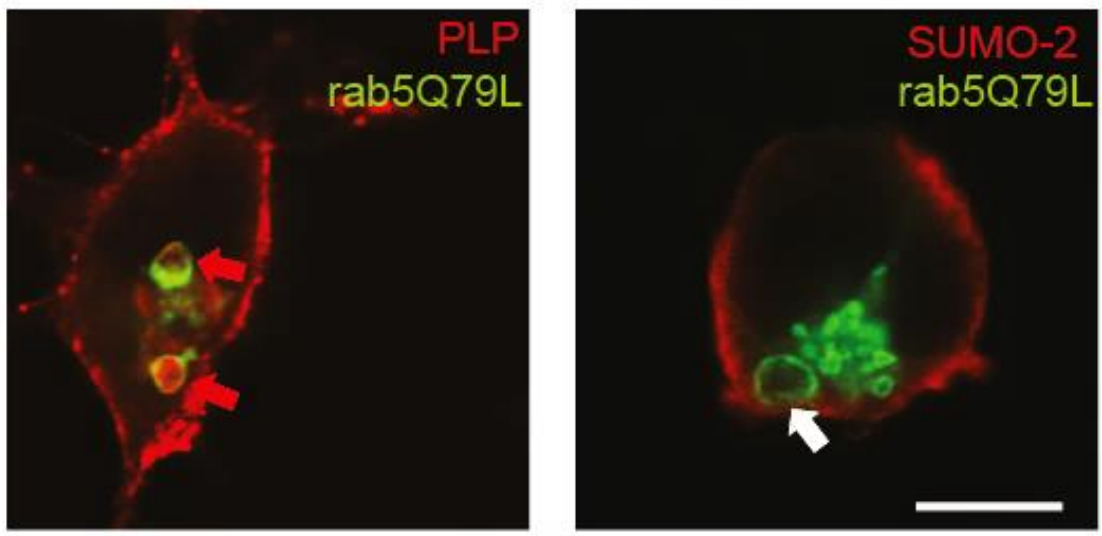

B
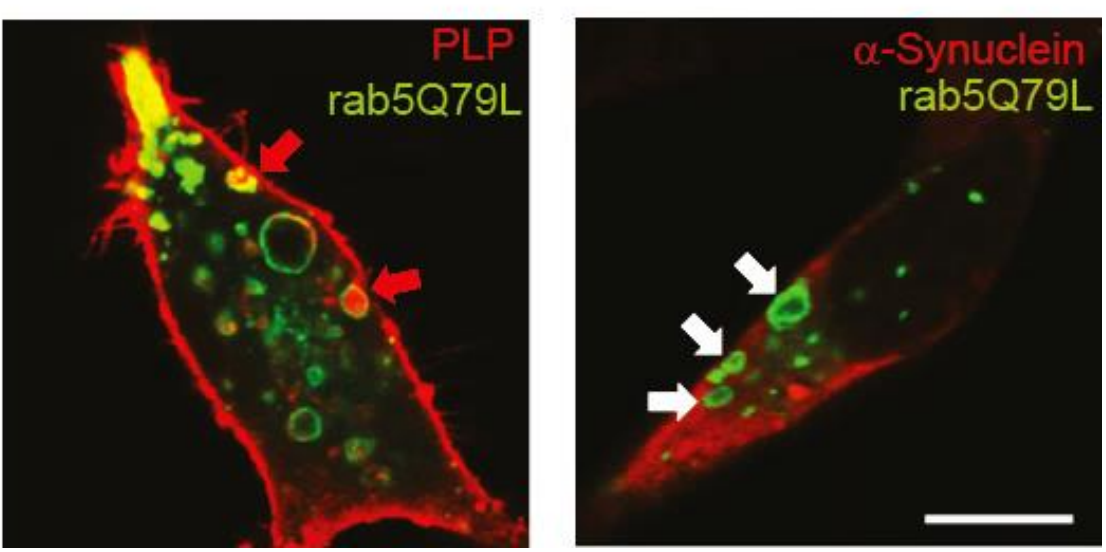

\section{Fig. 36: Subcellular distribution of SUMO-2 and $\alpha-S y n u c l e i n$}

(A) N2a cells were co-transfected with Rab5Q79L-GFP (green) and PLP-myc (red) or with Rab5Q79L-GFP (green) and Myc-SUMO-2 (red). (B) Same controls were transfected as indicated in (A) and N2a cells were cotransfected with or with Rab5Q79L-GFP (green) and Myc-a-Syn (red). Images were taken with by laser scanning confocal microscopy. Localization of positive control PLP-myc within Rab5Q79L endosomes are highlighted by red arrows. No localization for SUMO-2 and $\alpha$-Syn in Rab5Q79L endosomes was observed (white arrows right upper and lower panel). For each condition $n=20$ endosomes were analysed, scale bar $=10 \mu \mathrm{m}$. 


\section{Discussion}

\section{1. $\quad \alpha-S y n u c l e i n$ is localised in EVs in vitro}

Neuronal cells are able to release EVs to their environment (Fauré et al. 2006, Trajkovic et al. 2008). Consistent with previous studies (Danzer et al. 2012, Emmanouilidou et al. 2012), we could detect $\alpha$-Syn in EVs derived from N2a cells.

To clarify whether a-Syn is truly encapsulated in EVs or rather attached to the outer membrane, different trypsination assays were performed. Thus, we found that the vast bulk of $\alpha$-Syn recovered by ultracentrifugation resides within the EVs rather than being localised to the surface membrane of the vesicles. Our findings are contradictory to previous published results. Danzer et al. reported that only a small amount of $\alpha$-Syn in the EV preparation was affected by trypsin digestion, which lead to the assumption the $\alpha$-Syn is primarily localised to the outer vesicle membrane. However, EVs in this study were frozen after preparation and prior to trypsin digestion (Danzer, personal communication). In our assay, all EV preparations were digested immediately after preparation since freezing likely interferes with membrane integrity, making intravesicular protein accessible to trypsin. Under these experimental conditions, a-Syn was not degraded by trypsin and hence most likely localized within the vesicles.

Overexpression of exogenous a-Syn might artificially lead to its EV dependent release. To address this issue, we showed that cells also release endogenous $\alpha$-Syn with EVs. The detection of endogenous a-Syn is difficult due to the low expression levels, even in cell lysates (Lee et al. 2011). We have therefore isolated EVs from HEK cells and quantified intracellular and extracellular vesicle $\alpha$-Syn levels by the electrochemiluminescence assay described in section 2.2.4.4. For this approach we used HEK cells because this assay is only established for the detection of human $\alpha$-Syn and does not detect mouse $\alpha$-Syn (Kruse et al. 2012). (data not shown in the thesis). 


\subsection{Microvesicles or exosomes?}

For the purification of EVs a protocol with subsequent centrifugation steps including a final ultracentrifugation step was applied. By using this purification method, potential contaminations of the ultracentrifugation pellet ( $P-100)$ with protein aggregates (e.g. $\alpha-S y n$ which possibly aggregates in the cultured medium and could co-sediment during the ultracentrifugation process) and other vesicles (Mathivanan et al. 2012, Tauro et al. 2013) cannot be excluded.

Therefore, we additionally used sucrose gradient ultracentrifugation to eliminate contaminating proteins which might be non-specifically associated with EVs. However, for an accurate quantification of EV protein content we used ultracentrifugation rather than density gradient centrifugation, once we had established by sucrose gradient centrifugation that $\alpha$ Syn floats with EVs (Fig. 6 B).

Up to date it is not possible to discriminate between exosomes and (shedding) microvesicles in the size range of exosomes ( 100 nm) (Booth et al. 2006). It has been stated in several articles that "differences in properties such as size, morphology, buoyant density, and protein composition seem insufficient for a clear distinction" (Raposo et al. 2013) between both types of vesicles (Bobrie et al. 2011) (Simons et al. 2009) (Shen et al. 2011a). Therefore, exosomes and shedding microvesicles "remain mostly associated in the subcellular fractions isolated by differential centrifugation and by various types of gradient centrifugation"(Cocucci et al. 2009). Shen et al state that "differentiating between microvesicles and exosomes is problematic because (i) there is no known physical property or molecular marker that can unambiguously differentiate exosomes from microvesicles (Simons et al. 2009), and (ii) it is conceptually impossible to know where any particular secreted vesicle was made once it has left the cell (Shen et al. 2011a). The authors in this study therefore coined the umbrella term EMV for exosomes/microvesicles. Recently, due to the overlapping size range, the biochemical and physical properties of exosomes and microvesicles, it was recommended to use the term extracellular vesicle (EV) as "a generic term for all secreted vesicles" (Gould et al. 2013). Thus, we do not claim to distinguish between exosomes and microvesicles since clearly the methods we used do not allow to differentiate between both vesicle types. For this reason we use the term extracellular vesicles and not exosomes or microvesicles. Based on our ultracentrifugation/gradient density experiments, we cannot differentiate whether a-Syn and SUMO-2 are released via bona fide exosomes or shedding microvesicles.

Although the lack of $\alpha$-Syn and SUMO-2 in intraluminal vesicles after overexpression of rab5dn suggests that both proteins may be shedded from the plasma membrane, this experiment does not exclude that a-Syn and SUMO-2 are released by the exosomal/multivesicular body pathway (Fig. 36). 


\subsection{SUMO-2 interacts with phosphoinositols}

We show that SUMO-2 binds to PI3P with a $\mathrm{K}_{d}$ of $4 \mu \mathrm{M}$ and to $\mathrm{PI}(3,4,5) \mathrm{P}_{3}$ with a $\mathrm{K}_{d}$ of $6.1 \mu \mathrm{M}$ (Fig. 31). The similar lipid binding affinity of SUMO-2 for $\mathrm{PI}(3) \mathrm{P}$ and $\mathrm{PI}(3,4,5) \mathrm{P}_{3}$ would be compatible with both pathways, plasma membrane shedding and the MVB dependent SUMO-2 EV release. Phosphorylated inositol's can act as mediators of sorting cargo proteins to the ESRCT complex. The ESCRT-0 complex can be recruited to sites of intraluminal vesicle formation by PI(3)P binding of the Hrs FYVE domains (Hurley 2008).

In addition, further studies have revealed that $\mathrm{PI}(3,5) \mathrm{P} 2$ recruits cargo proteins to the ESCRT machinery localized at multivesicular endosomes (Friant et al. 2003, Whitley et al. 2003, Huotari et al. 2011). The cytosolic protein TyA can fuse with the $\mathrm{PI}(3,4,5) \mathrm{P} 3$-binding domain of AKT protein kinase and efficiently targets the protein to budding sites at the plasma membrane for extracellular vesicle release (Shen et al. 2011b). Both lipids, $\mathrm{PI}(3) \mathrm{P}$ and $\mathrm{P}(3,4,5) \mathrm{P}_{3}$ are known to recruit the ESCRT complex and interaction of SUMO-2 and SUMO-2 modified proteins with these lipids might explain the ESCRT dependent sorting of SUMO-2 into EVs. Of note, PI(3)P is known to be localized to endosomal membranes, while and $\mathrm{PI}(3,4,5) \mathrm{P}_{3}$ is predominantly enriched in plasma membranes (Henne et al. 2011).

\subsubsection{SUMO binding to plasma membranes is mediated by $\mathrm{PI}(3,4,5) \mathrm{P}_{3}$}

Based on our experiments with rab5Q79L overexpression (Fig. 36), it is likely that SUMO rather binds to the plasma membrane mediated by interaction with $\mathrm{PI}(3,4,5) \mathrm{P}_{3}$ than to endosomal membranes mediated by $\mathrm{PI}(3) \mathrm{P}$. The specificity of SUMO-2 binding to the plasma membrane (as opposed to endosomal $\mathrm{PI}(3) \mathrm{P}$ binding) may be caused by differences in the overall lipid composition of the inner plasma membrane leaflet compared to the endosomal membrane. One important difference regarding the lipid composition is the ratio of cholesterol to phospholipids which is significantly higher in the plasma membrane compared to endosomal membranes (van Meer et al. 2008). Interestingly, it has been shown that cholesterol can act as a spacer to segregate phosphoinositide lipids thereby reducing their electrostatic repulsion followed by a stabilization of membrane micro domains (Jiang et al. 2014). E.g., in the case of the tumor suppressor protein PTEN the presence of cholesterol enhances its binding to a variety of different phosphoinositides (Jiang et al. 2014). Thus, a preferential binding of SUMO to the plasma membrane may be mediated by a combination of high local cholesterol and the presence of $\mathrm{PI}(3,4,5) \mathrm{P} 3$. Clearly, further lipid binding experiments with different PIPs and a variety of PIP/cholesterol ratios would be required to unequivocally prove this assumption. 


\section{4. $\quad \alpha-S y n u c l e i n$ is localised in EVs in vivo}

At the beginning of this study it was not known whether a-Syn is present in EVs in vivo. EVs are abundant in different body fluids such as blood, plasma and urine (Keller et al. 2011) but have never been isolated from human CSF. We show for the first time, that EVs can be prepared from human CSF. From the presence of the CNS expressed proteins GluR1, 2, and 3 we conclude that these vesicles are at least partially derived from the CNS. We also deliver the first evidence of $\alpha$-Syn in extracellular vesicles in the human central nervous system in vivo (Fig. 7).

Interestingly, Danzer et al. could show that EVs contain a-Syn oligomers, that EV associated a-Syn is more likely to be taken up by target cells and is more neurotoxic than free, nonvesicular a-Syn oligomers (Emmanouilidou et al. 2010, Danzer et al. 2012, Luk et al. 2012a, Luk et al. 2012b, Mougenot et al. 2012).

Free $\alpha$-Syn may aggregate into oligomers and fibrils. Therefore, it could be difficult to avoid contaminations of free $\alpha$-Syn or its aggregated form when examining $\alpha$-Syn in Evs. To prove that a-Syn is indeed localised in EVs in vivo in human CSF, we provided evidence that $\alpha$-Syn can be detected in the "correct" sucrose gradient fractions. Due to the fact the sensitivity of Western blot analysis was not sufficient for this approach we decided to use an electrochemiluminescence based assay (2.2.4.4). We found that sucrose gradient ultracentrifugation of CSF derived EVs followed by electrochemiluminescence assay detection of $\alpha$-Syn (see section 3.1.2, Fig. 7 F) displayed a flotation behavior similar to the EV marker protein Flotilin-2 3.1.2, Fig. 7 D).

Thus, our findings that $\alpha$-Syn is present in EVs in vivo, strongly supports the hypothesis that EVs could contribute to disease propagation in PD and other synucleinopathies.

\subsection{Extracellular vesicles as carrier for pathogenic proteins}

EV transfer of pathogenic proteins has for example been shown for prion protein. The misfolded $\mathrm{PrP}^{\mathrm{Sc}}$ co-purifies with the cellular $\mathrm{PrP}^{\mathrm{c}}$ within $\mathrm{EVs}$ isolated from cell culture (Fevrier et al. 2004b, Vella et al. 2007), CSF (Vella et al. 2008) and blood (Robertson et al. 2006). PrP containing EVs can transmit infection to other cells, thus indicating a potential role for EVs mediated cell to cell spread of prion infection. A variety of neurodegenerative disorders are characterized by the misfolding and aggregation of proteins and peptides, including tau and $A \beta$ in Alzheimer's disease (Takahashi et al. 2015), $\mathrm{Cu} / \mathrm{Zn}$ superoxide dismutase (SOD1) and TAR-DNA binding protein 43 (TDP43) in Amyotrophic lateral sclerosis (ALS) (Pokrishevsky et al. 2012). 
Interestingly, these proteins have also been detected in EVs (Rajendran et al. 2006, Vingtdeux et al. 2012, Feneberg et al. 2014, Grad et al. 2014).

It is tempting to speculate that these proteins are packed into EVs in their aggregated misfolded form which would enable their pathological function as a nucleus to induce the aggregation of soluble proteins in recipient cells. In vitro, this has already been shown for EV associated $\alpha$-Syn (Danzer et al. 2012). It is interesting to note, that oligomerization of proteins alone is sufficient for their sorting and release with EVs (Booth et al. 2006). Therefore, it is feasible that especially pathological aggregates of proteins are enriched in EVs.

\subsubsection{Interneuronal spreading of $\alpha-S y n$ pathology}

Intracellular aggregates of $\alpha$-Syn, so called Lewy bodies (LB) are the neuropathological hallmark of Parkinson's disease (PD) and Lewy Body dementia (LBD) (Spillantini et al. 1998b). The progression of a-Syn pathology in PD seems to follow a stereotypical anatomical path through the brain (Braak et al. 2003). This, together with the emergence of LBs in transplanted embryonic nigral cells in PD patients lead to the assumption of interneuronal spreading of disease pathology (Kordower et al. 2008a, Li et al. 2008). Supporting this notion, cell to cell transfer of $\alpha$-Syn followed by aggregation of soluble $\alpha$-Syn in recipient cells was demonstrated in mouse brain and cell culture (Desplats et al. 2009, Hansen et al. 2011, Luk et al. 2012a, Luk et al. 2012b), however the transfer mode of pathogenic $\alpha$-Syn between neuronal cells is not known. Extracellular $\alpha-S y n$ has been proposed as a crucial mechanism for induction of pathological aggregate formation in previously healthy cells. Although $\alpha$-Syn does not contain a sorting signal for extracellular release, soluble and aggregated $\alpha$-Syn was detected in tissue culture medium and body fluids, such as brain interstitial fluid, plasma and CSF (El-Agnaf et al. 2003, Lee et al. 2005, El-Agnaf et al. 2006, Tokuda et al. 2010, Emmanouilidou et al. 2011, Hansson et al. 2014, Lee et al. 2014). 


\subsection{SUMO modification in neurodegenerative diseases}

We have identified SUMO modification as an important factor for EV release of cytosolic proteins. A growing body of evidence has linked SUMO modification to neurodegenerative diseases. E.g., Steffan and co-workers report an increased sumoylation of huntingtin in a Drosophila model which exacerbates neurodegeneration (Steffan et al. 2004). SUMOylation was also shown to induce the pathological hyper-phosphorylation of tau observed in Alzheimer's disease and also inhibits tau degradation (Luo et al. 2014) and sumoylated tau was also detected in AD brains (Luo et al. 2014). Importantly, an upregulation of tau SUMOylation was observed in vitro after exposure of neurons to toxic a (Luo et al. 2014). 


\section{Summary}

We hypothesized that cells release and transfer $\alpha$-Syn associated with EVs, followed by highly efficient internalization and induction of $\alpha$-Syn aggregation in previously healthy neurons. EVs of 40-100 nm diameter can either be derived from the multivesicular endosome (MVE) (exosomes) or shedded from the plasma membrane (microvesicles). Both types of vesicles are involved in the release of toxic cellular content and intercellular transfer of proteins, lipids and RNA and vesicular $\alpha$-Syn may be internalized more efficiently by recipient cells than the free protein and induce greater toxicity (Danzer et al. 2012).

We could show that $\alpha$-Syn is released with EVs and targeted to EVs by a completely novel mechanism based on SUMO modification. So far, ubiquitination had been regarded as an exclusive, necessary and sufficient signal for EV release of proteins. We could decipher the molecular mechanism of this novel sorting pathway, demonstrating that SUMO-dependent targeting to EVs depends on the endosomal sorting complex required for transport (ESCRT). Interestingly, the interaction of SUMO with ESCRT formation sites is mediated by SUMO binding to phosphoinositol containing membrane microdomains, most likely at the inner leaflet of the plasma membrane. By NMR spectrometry, we mapped the lipid interaction domain of SUMO to its hydrophobic cleft. Moreover, we could demonstrate that inhibition of SUMOylation by different genetic approaches strongly decreases the release of $\alpha$-Syn with EVs. In contrast, enhancing SUMOylation by co-expression of SUMO or fusion of SUMO to $\alpha$-Syn increased $\alpha$-Syn sorting to EVs. Similar to SUMO, the release of $\alpha$-Syn within EVs was inhibited by co-expression of a dominant-negative VPS4 mutant, indicating that EV sorting by SUMO modification is ESCRT-dependent.

Our findings are thus of highest relevance for the understanding of Parkinson's disease pathogenesis and progression at the molecular level. Moreover, we propose that SUMOdependent sorting constitutes a mechanism with more general implications for cell biology. 


\section{References}

1. M. Aalberts, F. M. van Dissel-Emiliani, N. P. van Adrichem, M. van Wijnen, M. H. Wauben, T. A. Stout, W. Stoorvogel, Identification of distinct populations of prostasomes that differentially express prostate stem cell antigen, annexin A1, and GLIPR2 in humans. Biology of reproduction 86(3):82, 81-88, (2012).

2. A. Abeliovich, Y. Schmitz, I. Farinas, D. Choi-Lundberg, W. H. Ho, P. E. Castillo, N. Shinsky, J. M. Verdugo, M. Armanini, A. Ryan, M. Hynes, H. Phillips, D. Sulzer, A. Rosenthal, Mice lacking alpha-synuclein display functional deficits in the nigrostriatal dopamine system. Neuron 25(1):239-252, (2000).

3. A. Aguzzi, L. Rajendran, The transcellular spread of cytosolic amyloids, prions, and prionoids. Neuron 64(6):783-790, (2009).

4. J. P. Anderson, D. E. Walker, J. M. Goldstein, R. de Laat, K. Banducci, R. J. Caccavello, R. Barbour, J. Huang, K. Kling, M. Lee, L. Diep, P. S. Keim, X. Shen, T. Chataway, M. G. Schlossmacher, P. Seubert, D. Schenk, S. Sinha, W. P. Gai, T. J. Chilcote, Phosphorylation of Ser-129 is the dominant pathological modification of alpha-synuclein in familial and sporadic Lewy body disease. The Journal of biological chemistry 281(40):2973929752, (2006).

5. E. Angot, J. A. Steiner, C. Hansen, J. Y. Li, P. Brundin, Are synucleinopathies prion-like disorders? Lancet neurology 9(11):1128-1138, (2010).

6. M. Babst, A protein's final ESCRT. Traffic 6(1):2-9, (2005).

7. M. Babst, MVB vesicle formation: ESCRT-dependent, ESCRT-independent and everything in between. Current opinion in cell biology 23(4):452-457, (2011).

8. M. Babst, B. Wendland, E. J. Estepa, S. D. Emr, The Vps4p AAA ATPase regulates membrane association of a $\mathrm{Vps}$ protein complex required for normal endosome function. The EMBO journal 17(11):2982-2993, (1998).

9. M. F. Baietti, Z. Zhang, E. Mortier, A. Melchior, G. Degeest, A. Geeraerts, Y. Ivarsson, F. Depoortere, C. Coomans, E. Vermeiren, P. Zimmermann, G. David, Syndecan-synteninALIX regulates the biogenesis of exosomes. Nature cell biology 14(7):677-685, (2012).

10. K. G. Baker, Y. Huang, H. McCann, W. P. Gai, P. H. Jensen, G. M. Halliday, P25alpha immunoreactive but alpha-synuclein immunonegative neuronal inclusions in multiple system atrophy. Acta neuropathologica 111(2):193-195, (2006).

11. T. Bartels, L. S. Ahlstrom, A. Leftin, F. Kamp, C. Haass, M. F. Brown, K. Beyer, The Nterminus of the intrinsically disordered protein alpha-synuclein triggers membrane binding and helix folding. Biophysical journal 99(7):2116-2124, (2010).

12. K. Beckett, S. Monier, L. Palmer, C. Alexandre, H. Green, E. Bonneil, G. Raposo, P. Thibault, R. Le Borgne, J. P. Vincent, Drosophila S2 cells secrete wingless on exosome-like vesicles but the wingless gradient forms independently of exosomes. Traffic 14(1):82-96, (2013).

13. S. A. Bellingham, B. B. Guo, B. M. Coleman, A. F. Hill, Exosomes: vehicles for the transfer of toxic proteins associated with neurodegenerative diseases? Frontiers in physiology $\mathbf{3}(124$, (2012).

14. J. T. Bendor, T. P. Logan, R. H. Edwards, The function of alpha-synuclein. Neuron 79(6):1044-1066, (2013).

15. V. Bernier-Villamor, D. A. Sampson, M. J. Matunis, C. D. Lima, Structural basis for E2mediated SUMO conjugation revealed by a complex between ubiquitin-conjugating enzyme Ubc9 and RanGAP1. Cell 108(3):345-356, (2002).

16. U. Bertsch, K. F. Winklhofer, T. Hirschberger, J. Bieschke, P. Weber, F. U. Hartl, P. Tavan, J. Tatzelt, H. A. Kretzschmar, A. Giese, Systematic identification of antiprion drugs by high-throughput screening based on scanning for intensely fluorescent targets. Journal of virology 79(12):7785-7791, (2005). 
17. J. Bieschke, A. Giese, W. Schulz-Schaeffer, I. Zerr, S. Poser, M. Eigen, H. Kretzschmar, Ultrasensitive detection of pathological prion protein aggregates by dual-color scanning for intensely fluorescent targets. Proceedings of the National Academy of Sciences of the United States of America 97(10):5468-5473, (2000).

18. N. Bishop, P. Woodman, ATPase-defective mammalian VPS4 localizes to aberrant endosomes and impairs cholesterol trafficking. Molecular biology of the cell 11(1):227-239, (2000).

19. A. Bjorklund, S. B. Dunnett, P. Brundin, A. J. Stoessl, C. R. Freed, R. E. Breeze, M. Levivier, M. Peschanski, L. Studer, R. Barker, Neural transplantation for the treatment of Parkinson's disease. Lancet neurology 2(7):437-445, (2003).

20. A. Bobrie, M. Colombo, G. Raposo, C. Thery, Exosome secretion: molecular mechanisms and roles in immune responses. Traffic 12(12):1659-1668, (2011).

21. A. M. Bodles, D. J. Guthrie, B. Greer, G. B. Irvine, Identification of the region of non-Abeta component (NAC) of Alzheimer's disease amyloid responsible for its aggregation and toxicity. Journal of neurochemistry 78(2):384-395, (2001).

22. C. R. Bodner, A. S. Maltsev, C. M. Dobson, A. Bax, Differential phospholipid binding of alpha-synuclein variants implicated in Parkinson's disease revealed by solution NMR spectroscopy. Biochemistry 49(5):862-871, (2010).

23. V. Bonifati, P. Rizzu, F. Squitieri, E. Krieger, N. Vanacore, J. C. van Swieten, A. Brice, C. M. van Duijn, B. Oostra, G. Meco, P. Heutink, DJ-1( PARK7), a novel gene for autosomal recessive, early onset parkinsonism. Neurological sciences : official journal of the Italian Neurological Society and of the Italian Society of Clinical Neurophysiology 24(3):159-160, (2003).

24. A. M. Booth, Y. Fang, J. K. Fallon, J. M. Yang, J. E. Hildreth, S. J. Gould, Exosomes and HIV Gag bud from endosome-like domains of the T cell plasma membrane. The Journal of cell biology 172(6):923-935, (2006).

25. H. Braak, K. Del Tredici, U. Rub, R. A. de Vos, E. N. Jansen Steur, E. Braak, Staging of brain pathology related to sporadic Parkinson's disease. Neurobiology of aging 24(2):197-211, (2003).

26. C. Braicu, C. Tomuleasa, P. Monroig, A. Cucuianu, I. Berindan-Neagoe, G. A. Calin, Exosomes as divine messengers: are they the Hermes of modern molecular oncology? Cell death and differentiation 22(1):34-45, (2015).

27. J. Braman, C. Papworth, A. Greener, Site-directed mutagenesis using double-stranded plasmid DNA templates. Methods Mol Biol 57(31-44, (1996).

28. J. F. Brouwers, M. Aalberts, J. W. Jansen, G. van Niel, M. H. Wauben, T. A. Stout, J. B. Helms, W. Stoorvogel, Distinct lipid compositions of two types of human prostasomes. Proteomics 13(10-11):1660-1666, (2013).

29. P. Brundin, J. Y. Li, J. L. Holton, O. Lindvall, T. Revesz, Research in motion: the enigma of Parkinson's disease pathology spread. Nature reviews. Neuroscience 9(10):741-745, (2008).

30. V. L. Buchman, H. J. Hunter, L. G. Pinon, J. Thompson, E. M. Privalova, N. N. Ninkina, A. M. Davies, Persyn, a member of the synuclein family, has a distinct pattern of expression in the developing nervous system. The Journal of neuroscience : the official journal of the Society for Neuroscience 18(22):9335-9341, (1998).

31. M. P. Caby, D. Lankar, C. Vincendeau-Scherrer, G. Raposo, C. Bonnerot, Exosomal-like vesicles are present in human blood plasma. International immunology 17(7):879-887, (2005).

32. M. Chaineau, L. Danglot, T. Galli, Multiple roles of the vesicular-SNARE TI-VAMP in postGolgi and endosomal trafficking. FEBS letters 583(23):3817-3826, (2009).

33. E. Cocucci, G. Racchetti, J. Meldolesi, Shedding microvesicles: artefacts no more. Trends in cell biology 19(2):43-51, (2009).

34. M. Colombo, G. Raposo, C. Thery, Biogenesis, secretion, and intercellular interactions of exosomes and other extracellular vesicles. Annual review of cell and developmental biology 30(255-289, (2014). 
35. J. Conde-Vancells, E. Rodriguez-Suarez, N. Embade, D. Gil, R. Matthiesen, M. Valle, F. Elortza, S. C. Lu, J. M. Mato, J. M. Falcon-Perez, Characterization and comprehensive proteome profiling of exosomes secreted by hepatocytes. Journal of proteome research 7(12):5157-5166, (2008).

36. M. R. Cookson, The biochemistry of Parkinson's disease. Annual review of biochemistry 74(29-52, (2005).

37. A. A. Cooper, A. D. Gitler, A. Cashikar, C. M. Haynes, K. J. Hill, B. Bhullar, K. Liu, K. Xu, K. E. Strathearn, F. Liu, S. Cao, K. A. Caldwell, G. A. Caldwell, G. Marsischky, R. D. Kolodner, J. Labaer, J. C. Rochet, N. M. Bonini, S. Lindquist, Alpha-synuclein blocks ERGolgi traffic and Rab1 rescues neuron loss in Parkinson's models. Science 313(5785):324328, (2006).

38. O. Corti, S. Lesage, A. Brice, What genetics tells us about the causes and mechanisms of Parkinson's disease. Physiological reviews 91(4):1161-1218, (2011).

39. M. Costanzo, S. Abounit, L. Marzo, A. Danckaert, Z. Chamoun, P. Roux, C. Zurzolo, Transfer of polyglutamine aggregates in neuronal cells occurs in tunneling nanotubes. Journal of cell science 126(Pt 16):3678-3685, (2013).

40. S. R. Danielson, J. M. Held, B. Schilling, M. Oo, B. W. Gibson, J. K. Andersen, Preferentially increased nitration of alpha-synuclein at tyrosine-39 in a cellular oxidative model of Parkinson's disease. Analytical chemistry 81(18):7823-7828, (2009).

41. K. M. Danzer, L. R. Kranich, W. P. Ruf, O. Cagsal-Getkin, A. R. Winslow, L. Zhu, C. R. Vanderburg, P. J. McLean, Exosomal cell-to-cell transmission of alpha synuclein oligomers. Molecular neurodegeneration 7(42, (2012).

42. A. de Gassart, C. Geminard, B. Fevrier, G. Raposo, M. Vidal, Lipid raft-associated protein sorting in exosomes. Blood 102(13):4336-4344, (2003).

43. L. M. de Lau, M. M. Breteler, Epidemiology of Parkinson's disease. Lancet neurology 5(6):525-535, (2006).

44. P. Desplats, H. J. Lee, E. J. Bae, C. Patrick, E. Rockenstein, L. Crews, B. Spencer, E. Masliah, S. J. Lee, Inclusion formation and neuronal cell death through neuron-to-neuron transmission of alpha-synuclein. Proceedings of the National Academy of Sciences of the United States of America 106(31):13010-13015, (2009).

45. J. M. Desterro, M. S. Rodriguez, G. D. Kemp, R. T. Hay, Identification of the enzyme required for activation of the small ubiquitin-like protein SUMO-1. The Journal of biological chemistry 274(15):10618-10624, (1999).

46. J. M. Desterro, J. Thomson, R. T. Hay, Ubch9 conjugates SUMO but not ubiquitin. FEBS letters 417(3):297-300, (1997).

47. P. C. Donaghy, I. G. McKeith, The clinical characteristics of dementia with Lewy bodies and a consideration of prodromal diagnosis. Alzheimer's research \& therapy 6(4):46, (2014).

48. V. Dorval, P. E. Fraser, Small ubiquitin-like modifier (SUMO) modification of natively unfolded proteins tau and alpha-synuclein. The Journal of biological chemistry 281(15):9919-9924, (2006).

49. V. Dorval, P. E. Fraser, SUMO on the road to neurodegeneration. Biochimica et biophysica acta 1773(6):694-706, (2007a).

50. V. Dorval, M. J. Mazzella, P. M. Mathews, R. T. Hay, P. E. Fraser, Modulation of Abeta generation by small ubiquitin-like modifiers does not require conjugation to target proteins. The Biochemical journal 404(2):309-316, (2007b).

51. L. M. Duncan, S. Piper, R. B. Dodd, M. K. Saville, C. M. Sanderson, J. P. Luzio, P. J. Lehner, Lysine-63-linked ubiquitination is required for endolysosomal degradation of class I molecules. The EMBO journal 25(8):1635-1645, (2006).

52. O. M. El-Agnaf, A. M. Bodles, D. J. Guthrie, P. Harriott, G. B. Irvine, The N-terminal region of non-A beta component of Alzheimer's disease amyloid is responsible for its tendency to assume beta-sheet and aggregate to form fibrils. European journal of biochemistry / FEBS 258(1):157-163, (1998). 
53. O. M. El-Agnaf, S. A. Salem, K. E. Paleologou, L. J. Cooper, N. J. Fullwood, M. J. Gibson, M. D. Curran, J. A. Court, D. M. Mann, S. Ikeda, M. R. Cookson, J. Hardy, D. Allsop, Alpha-synuclein implicated in Parkinson's disease is present in extracellular biological fluids, including human plasma. FASEB J 17(13):1945-1947, (2003).

54. O. M. El-Agnaf, S. A. Salem, K. E. Paleologou, M. D. Curran, M. J. Gibson, J. A. Court, M. G. Schlossmacher, D. Allsop, Detection of oligomeric forms of alpha-synuclein protein in human plasma as a potential biomarker for Parkinson's disease. FASEB J 20(3):419-425, (2006).

55. E. Emmanouilidou, D. Elenis, T. Papasilekas, G. Stranjalis, K. Gerozissis, P. C. loannou, K. Vekrellis, Assessment of alpha-synuclein secretion in mouse and human brain parenchyma. PLoS One 6(7):e22225, (2011).

56. E. Emmanouilidou, K. Melachroinou, T. Roumeliotis, S. D. Garbis, M. Ntzouni, L. H. Margaritis, L. Stefanis, K. Vekrellis, Cell-produced alpha-synuclein is secreted in a calciumdependent manner by exosomes and impacts neuronal survival. The Journal of neuroscience : the official journal of the Society for Neuroscience 30(20):6838-6851, (2010).

57. E. Emmanouilidou, T. Papasilekas, K. Gerozissis, P. C. loannou, K. Vekrellis, Investigation of the mechanisms of alpha-synuclein secretion in vivo. Movement Disord 27(S479-S479, (2012).

58. C. M. Fader, D. G. Sanchez, M. B. Mestre, M. I. Colombo, TI-VAMP/VAMP7 and VAMP3/cellubrevin: two V-SNARE proteins involved in specific steps of the autophagy/multivesicular body pathways. Biochimica et biophysica acta 1793(12):1901-1916, (2009).

59. L. J. Falomir-Lockhart, G. R. Franchini, M. X. Guerbi, J. Storch, B. Corsico, Interaction of enterocyte FABPs with phospholipid membranes: clues for specific physiological roles. Biochimica et biophysica acta 1811(7-8):452-459, (2011).

60. Y. Fang, N. Wu, X. Gan, W. Yan, J. C. Morrell, S. J. Gould, Higher-order oligomerization targets plasma membrane proteins and HIV gag to exosomes. PLoS biology 5(6):e158, (2007).

61. J. Fauré, G. Lachenal, M. Court, J. Hirrlinger, C. Chatellard-Causse, B. Blot, J. Grange, G. Schoehn, Y. Goldberg, V. Boyer, F. Kirchhoff, G. Raposo, J. Garin, R. Sadoul, Exosomes are released by cultured cortical neurones. Molecular and cellular neurosciences 31(4):642-648, (2006).

62. E. Feneberg, P. Steinacker, S. Lehnert, A. Schneider, P. Walther, D. R. Thal, M. Linsenmeier, A. C. Ludolph, M. Otto, Limited role of free TDP-43 as a diagnostic tool in neurodegenerative diseases. Amyotrophic lateral sclerosis \& frontotemporal degeneration 15(5-6):351-356, (2014).

63. B. Fevrier, G. Raposo, Exosomes: endosomal-derived vesicles shipping extracellular messages. Current opinion in cell biology 16(4):415-421, (2004a).

64. B. Fevrier, D. Vilette, F. Archer, D. Loew, W. Faigle, M. Vidal, H. Laude, G. Raposo, Cells release prions in association with exosomes. Proceedings of the National Academy of Sciences of the United States of America 101(26):9683-9688, (2004b).

65. S. Friant, E. I. Pecheur, A. Eugster, F. Michel, Y. Lefkir, D. Nourrisson, F. Letourneur, Ent3p Is a Ptdlns(3,5)P2 effector required for protein sorting to the multivesicular body. Developmental cell 5(3):499-511, (2003).

66. H. Fujiwara, M. Hasegawa, N. Dohmae, A. Kawashima, E. Masliah, M. S. Goldberg, J. Shen, K. Takio, T. Iwatsubo, alpha-Synuclein is phosphorylated in synucleinopathy lesions. Nature cell biology 4(2):160-164, (2002).

67. J. R. Gareau, C. D. Lima, The SUMO pathway: emerging mechanisms that shape specificity, conjugation and recognition. 11(12):861-871, (2010).

68. R. Gastpar, M. Gehrmann, M. A. Bausero, A. Asea, C. Gross, J. A. Schroeder, G. Multhoff, Heat shock protein 70 surface-positive tumor exosomes stimulate migratory and cytolytic activity of natural killer cells. Cancer research 65(12):5238-5247, (2005).

69. R. Geiss-Friedlander, F. Melchior, Concepts in sumoylation: a decade on. Nature reviews. Molecular cell biology 8(12):947-956, (2007). 
70. B. I. Giasson, J. E. Duda, I. V. Murray, Q. Chen, J. M. Souza, H. I. Hurtig, H. Ischiropoulos, J. Q. Trojanowski, V. M. Lee, Oxidative damage linked to neurodegeneration by selective alpha-synuclein nitration in synucleinopathy lesions. Science 290(5493):985-989, (2000).

71. B. I. Giasson, I. V. Murray, J. Q. Trojanowski, V. M. Lee, A hydrophobic stretch of 12 amino acid residues in the middle of alpha-synuclein is essential for filament assembly. The Journal of biological chemistry 276(4):2380-2386, (2001).

72. A. Giese, B. Bader, J. Bieschke, G. Schaffar, S. Odoy, P. J. Kahle, C. Haass, H. Kretzschmar, Single particle detection and characterization of synuclein co-aggregation. Biochemical and biophysical research communications 333(4):1202-1210, (2005).

73. A. Giese, J. Bieschke, M. Eigen, H. A. Kretzschmar, Putting prions into focus: application of single molecule detection to the diagnosis of prion diseases. Archives of virology. Supplementum 16):161-171, (2000).

74. A. Giese, J. Levin, U. Bertsch, H. Kretzschmar, Effect of metal ions on de novo aggregation of full-length prion protein. Biochemical and biophysical research communications 320(4):1240-1246, (2004).

75. J. M. Gil, A. C. Rego, Mechanisms of neurodegeneration in Huntington's disease. The European journal of neuroscience 27(11):2803-2820, (2008).

76. P. K. Giri, J. S. Schorey, Exosomes derived from M. Bovis BCG infected macrophages activate antigen-specific CD4+ and CD8+ T cells in vitro and in vivo. PloS one 3(6):e2461, (2008).

77. A. D. Gitler, B. J. Bevis, J. Shorter, K. E. Strathearn, S. Hamamichi, L. J. Su, K. A. Caldwell, G. A. Caldwell, J. C. Rochet, J. M. McCaffery, C. Barlowe, S. Lindquist, The Parkinson's disease protein alpha-synuclein disrupts cellular Rab homeostasis. Proceedings of the National Academy of Sciences of the United States of America 105(1):145-150, (2008).

78. C. B. Gocke, H. Yu, J. Kang, Systematic identification and analysis of mammalian small ubiquitin-like modifier substrates. The Journal of biological chemistry 280(6):5004-5012, (2005).

79. M. Goedert, B. Falcon, F. Clavaguera, M. Tolnay, Prion-like mechanisms in the pathogenesis of tauopathies and synucleinopathies. Current neurology and neuroscience reports 14(11):495, (2014).

80. G. Gomori, A modification of the colorimetric phosphorus determination for use with the photoelectric colorimeter. J. Lab. Clin. Med 27(955):1941-1942, (1942).

81. S. Goncalves, T. F. Outeiro, Assessing the subcellular dynamics of alpha-synuclein using photoactivation microscopy. Molecular neurobiology 47(3):1081-1092, (2013).

82. L. Gong, B. Li, S. Millas, E. T. Yeh, Molecular cloning and characterization of human AOS1 and UBA2, components of the sentrin-activating enzyme complex. FEBS letters 448(1):185189, (1999).

83. S. J. Gould, G. Raposo, As we wait: coping with an imperfect nomenclature for extracellular vesicles. Journal of extracellular vesicles 2((2013).

84. L. I. Grad, E. Pokrishevsky, J. M. Silverman, N. R. Cashman, Exosome-dependent and independent mechanisms are involved in prion-like transmission of propagated $\mathrm{Cu} / \mathrm{Zn}$ superoxide dismutase misfolding. Prion 8(5):331-335, (2014).

85. F. L. Graham, A. J. van der Eb, A new technique for the assay of infectivity of human adenovirus 5 DNA. Virology 52(2):456-467, (1973a).

86. F. L. Graham, A. J. van der Eb, Transformation of rat cells by DNA of human adenovirus 5 . Virology 54(2):536-539, (1973b).

87. J. C. Gross, V. Chaudhary, K. Bartscherer, M. Boutros, Active Wnt proteins are secreted on exosomes. Nature cell biology 14(10):1036-1045, (2012).

88. D. Guo, M. Li, Y. Zhang, P. Yang, S. Eckenrode, D. Hopkins, W. Zheng, S. Purohit, R. H. Podolsky, A. Muir, J. Wang, Z. Dong, T. Brusko, M. Atkinson, P. Pozzilli, A. Zeidler, L. J. Raffel, C. O. Jacob, Y. Park, M. Serrano-Rios, M. T. Larrad, Z. Zhang, H. J. Garchon, J. F. Bach, J. I. Rotter, J. X. She, C. Y. Wang, A functional variant of SUMO4, a new I kappa B alpha modifier, is associated with type 1 diabetes. Nature genetics 36(8):837-841, (2004). 
89. P. Gutwein, A. Stoeck, S. Riedle, D. Gast, S. Runz, T. P. Condon, A. Marme, M. C. Phong, O. Linderkamp, A. Skorokhod, P. Altevogt, Cleavage of L1 in exosomes and apoptotic membrane vesicles released from ovarian carcinoma cells. Clinical cancer research : an official journal of the American Association for Cancer Research 11(7):2492-2501, (2005).

90. B. György, T. G. Szabo, M. Pasztoi, Z. Pal, P. Misjak, B. Aradi, V. Laszlo, E. Pallinger, E. Pap, A. Kittel, G. Nagy, A. Falus, E. I. Buzas, Membrane vesicles, current state-of-the-art: emerging role of extracellular vesicles. Cellular and molecular life sciences : CMLS 68(16):2667-2688, (2011).

91. C. Haan, I. Behrmann, A cost effective non-commercial ECL-solution for Western blot detections yielding strong signals and low background. Journal of immunological methods 318(1-2):11-19, (2007).

92. K. Haglund, I. Dikic, Ubiquitylation and cell signaling. The EMBO journal 24(19):3353-3359, (2005).

93. J. T. Hannich, A. Lewis, M. B. Kroetz, S. J. Li, H. Heide, A. Emili, M. Hochstrasser, Defining the SUMO-modified proteome by multiple approaches in Saccharomyces cerevisiae. The Journal of biological chemistry 280(6):4102-4110, (2005).

94. C. Hansen, E. Angot, A. L. Bergstrom, J. A. Steiner, L. Pieri, G. Paul, T. F. Outeiro, R. Melki, P. Kallunki, K. Fog, J. Y. Li, P. Brundin, alpha-Synuclein propagates from mouse brain to grafted dopaminergic neurons and seeds aggregation in cultured human cells. The Journal of clinical investigation 121(2):715-725, (2011).

95. O. Hansson, S. Hall, A. Ohrfelt, H. Zetterberg, K. Blennow, L. Minthon, K. Nagga, E. Londos, S. Varghese, N. K. Majbour, A. Al-Hayani, O. M. El-Agnaf, Levels of cerebrospinal fluid alpha-synuclein oligomers are increased in Parkinson's disease with dementia and dementia with Lewy bodies compared to Alzheimer's disease. Alzheimer's research \& therapy 6(3):25, (2014).

96. C. Harding, J. Heuser, P. Stahl, Receptor-mediated endocytosis of transferrin and recycling of the transferrin receptor in rat reticulocytes. The Journal of cell biology 97(2):329-339, (1983).

97. C. Harding, J. Heuser, P. Stahl, Endocytosis and intracellular processing of transferrin and colloidal gold-transferrin in rat reticulocytes: demonstration of a pathway for receptor shedding. European journal of cell biology 35(2):256-263, (1984).

98. J. Hardy, Expression of normal sequence pathogenic proteins for neurodegenerative disease contributes to disease risk: 'permissive templating' as a general mechanism underlying neurodegeneration. Biochemical Society transactions 33(Pt 4):578-581, (2005).

99. R. T. Hay, SUMO: a history of modification. Molecular cell 18(1):1-12, (2005).

100. R. T. Hay, SUMO-specific proteases: a twist in the tail. Trends in cell biology 17(8):370-376, (2007).

101. C. M. Hecker, M. Rabiller, K. Haglund, P. Bayer, I. Dikic, Specification of SUMO1- and SUMO2-interacting motifs. The Journal of biological chemistry 281(23):16117-16127, (2006).

102. W. M. Henne, N. J. Buchkovich, S. D. Emr, The ESCRT pathway. Developmental cell 21(1):77-91, (2011).

103. C. Hess, S. Sadallah, A. Hefti, R. Landmann, J. A. Schifferli, Ectosomes released by human neutrophils are specialized functional units. J Immunol 163(8):4564-4573, (1999).

104. L. Hicke, R. Dunn, Regulation of membrane protein transport by ubiquitin and ubiquitinbinding proteins. Annual review of cell and developmental biology 19(141-172, (2003).

105. M. Hochstrasser, SP-RING for SUMO: new functions bloom for a ubiquitin-like protein. Cell 107(1):5-8, (2001).

106. M. Hochstrasser, Origin and function of ubiquitin-like proteins. 458(7237):422-429, (2009).

107. T. Högen, J. Levin, F. Schmidt, M. Caruana, N. Vassallo, H. Kretzschmar, K. Botzel, F. Kamp, A. Giese, Two different binding modes of alpha-synuclein to lipid vesicles depending on its aggregation state. Biophysical journal 102(7):1646-1655, (2012).

108. P. A. Holme, N. O. Solum, F. Brosstad, M. Roger, M. Abdelnoor, Demonstration of plateletderived microvesicles in blood from patients with activated coagulation and fibrinolysis using a filtration technique and western blotting. Thrombosis and haemostasis 72(5):666-671, (1994). 
109. C. Huang, T. E. Thompson, Preparation of homogeneous, single-walled phosphatidylcholine vesicles. Methods in enzymology 32(485-489, (1974).

110. F. Huang, D. Kirkpatrick, X. Jiang, S. Gygi, A. Sorkin, Differential regulation of EGF receptor internalization and degradation by multiubiquitination within the kinase domain. Molecular cell 21(6):737-748, (2006).

111. Y. Huang, Y. J. Song, K. Murphy, J. L. Holton, T. Lashley, T. Revesz, W. P. Gai, G. M. Halliday, LRRK2 and parkin immunoreactivity in multiple system atrophy inclusions. Acta neuropathologica 116(6):639-646, (2008).

112. M. P. Hunter, N. Ismail, X. Zhang, B. D. Aguda, E. J. Lee, L. Yu, T. Xiao, J. Schafer, M. L. Lee, T. D. Schmittgen, S. P. Nana-Sinkam, D. Jarjoura, C. B. Marsh, Detection of microRNA expression in human peripheral blood microvesicles. PloS one 3(11):e3694, (2008).

113. J. Huotari, A. Helenius, Endosome maturation. The EMBO journa/ 30(17):3481-3500, (2011).

114. J. H. Hurley, ESCRT complexes and the biogenesis of multivesicular bodies. Current opinion in cell biology 20(1):4-11, (2008).

115. J. H. Hurley, The ESCRT complexes. Critical reviews in biochemistry and molecular biology 45(6):463-487, (2010).

116. K. W. Hwang, T. J. Won, H. Kim, H. J. Chun, T. Chun, Y. Park, Erratum to "Characterization of the regulatory roles of the SUMO. Diabetes/metabolism research and reviews 28(2):196202, (2012).

117. M. C. Irizarry, T. W. Kim, M. McNamara, R. E. Tanzi, J. M. George, D. F. Clayton, B. T. Hyman, Characterization of the precursor protein of the non-A beta component of senile plaques (NACP) in the human central nervous system. Journal of neuropathology and experimental neurology 55(8):889-895, (1996).

118. A. Iwai, E. Masliah, M. Yoshimoto, N. Ge, L. Flanagan, H. R. de Silva, A. Kittel, T. Saitoh, The precursor protein of non-A $\beta$ component of Alzheimer's disease amyloid is a presynaptic protein of the central nervous system. Neuron 14(2):467-475, (1995).

119. R. Jakes, M. G. Spillantini, M. Goedert, Identification of two distinct synucleins from human brain. FEBS letters 345(1):27-32, (1994).

120. P. Jenner, Oxidative stress in Parkinson's disease. Annals of neurology 53 Suppl 3(S26-36; discussion S36-28, (2003).

121. T. Jia, Y. E. Liu, J. Liu, Y. E. Shi, Stimulation of breast cancer invasion and metastasis by synuclein gamma. Cancer research 59(3):742-747, (1999).

122. Z. Jiang, R. E. Redfern, Y. Isler, A. H. Ross, A. Gericke, Cholesterol stabilizes fluid phosphoinositide domains. Chemistry and physics of lipids 182(52-61, (2014).

123. H. Jin, K. Ishikawa, T. Tsunemi, T. Ishiguro, T. Amino, H. Mizusawa, Analyses of copy number and mRNA expression level of the alpha-synuclein gene in multiple system atrophy. Journal of medical and dental sciences 55(1):145-153, (2008).

124. E. S. Johnson, Protein modification by SUMO. Annual review of biochemistry 73(355-382, (2004).

125. E. S. Johnson, G. Blobel, Ubc9p is the conjugating enzyme for the ubiquitin-like protein Smt3p. Journal of Biological Chemistry 272(43):26799-26802, (1997).

126. E. S. Johnson, A. A. Gupta, An E3-like factor that promotes SUMO conjugation to the yeast septins. Cell 106(6):735-744, (2001).

127. R. M. Johnstone, M. Adam, J. R. Hammond, L. Orr, C. Turbide, Vesicle formation during reticulocyte maturation. Association of plasma membrane activities with released vesicles (exosomes). The Journal of biological chemistry 262(19):9412-9420, (1987).

128. H. Karube, M. Sakamoto, S. Arawaka, S. Hara, H. Sato, C. H. Ren, S. Goto, S. Koyama, M. Wada, T. Kawanami, K. Kurita, T. Kato, N-terminal region of alpha-synuclein is essential for the fatty acid-induced oligomerization of the molecules. FEBS letters 582(25-26):36933700, (2008).

129. S. Keller, J. Ridinger, A. K. Rupp, J. W. Janssen, P. Altevogt, Body fluid derived exosomes as a novel template for clinical diagnostics. Journal of translational medicine 9(86, (2011). 
130. S. Keller, M. P. Sanderson, A. Stoeck, P. Altevogt, Exosomes: from biogenesis and secretion to biological function. Immunology letters 107(2):102-108, (2006).

131. W. S. Kim, K. Kagedal, G. M. Halliday, Alpha-synuclein biology in Lewy body diseases. Alzheimer's research \& therapy 6(5):73, (2014).

132. C. Klein, K. Lohmann-Hedrich, E. Rogaeva, M. G. Schlossmacher, A. E. Lang, Deciphering the role of heterozygous mutations in genes associated with parkinsonism. Lancet neurology 6(7):652-662, (2007).

133. V. S. Kokhan, M. A. Afanasyeva, G. I. Van'kin, alpha-Synuclein knockout mice have cognitive impairments. Behavioural brain research 231(1):226-230, (2012).

134. J. H. Kordower, Y. Chu, R. A. Hauser, T. B. Freeman, C. W. Olanow, Lewy body-like pathology in long-term embryonic nigral transplants in Parkinson's disease. Nature medicine 14(5):504-506, (2008a).

135. J. H. Kordower, Y. Chu, R. A. Hauser, C. W. Olanow, T. B. Freeman, Transplanted dopaminergic neurons develop PD pathologic changes: a second case report. Movement disorders : official journal of the Movement Disorder Society 23(16):2303-2306, (2008b).

136. J. H. Kordower, T. B. Freeman, B. J. Snow, F. J. Vingerhoets, E. J. Mufson, P. R. Sanberg, R. A. Hauser, D. A. Smith, G. M. Nauert, D. P. Perl, et al., Neuropathological evidence of graft survival and striatal reinnervation after the transplantation of fetal mesencephalic tissue in a patient with Parkinson's disease. The New England journal of medicine 332(17):1118-1124, (1995).

137. R. Krüger, W. Kuhn, T. Muller, D. Woitalla, M. Graeber, S. Kosel, H. Przuntek, J. T. Epplen, L. Schols, O. Riess, Ala30Pro mutation in the gene encoding alpha-synuclein in Parkinson's disease. Nature genetics 18(2):106-108, (1998).

138. P. Krumova, E. Meulmeester, M. Garrido, M. Tirard, H. H. Hsiao, G. Bossis, H. Urlaub, M. Zweckstetter, S. Kügler, F. Melchior, M. Bähr, J. H. Weishaupt, Sumoylation inhibits alphasynuclein aggregation and toxicity. The Journal of cell biology 194(1):49-60, (2011).

139. N. Kruse, W. J. Schulz-Schaeffer, M. G. Schlossmacher, B. Mollenhauer, Development of electrochemiluminescence-based singleplex and multiplex assays for the quantification of alpha-synuclein and other proteins in cerebrospinal fluid. Methods 56(4):514-518, (2012).

140. T. Kuwahara, A. Koyama, S. Koyama, S. Yoshina, C. H. Ren, T. Kato, S. Mitani, T. Iwatsubo, A systematic RNAi screen reveals involvement of endocytic pathway in neuronal dysfunction in alpha-synuclein transgenic C. elegans. Human molecular genetics 17(19):29973009, (2008).

141. U. K. Laemmli, Cleavage of structural proteins during the assembly of the head of bacteriophage T4. Nature 227(5259):680-685, (1970).

142. K. Laulagnier, C. Motta, S. Hamdi, S. Roy, F. Fauvelle, J. F. Pageaux, T. Kobayashi, J. P. Salles, B. Perret, C. Bonnerot, M. Record, Mast cell- and dendritic cell-derived exosomes display a specific lipid composition and an unusual membrane organization. The Biochemical journal 380(Pt 1):161-171, (2004).

143. C. Lavedan, The synuclein family. Genome research 8(9):871-880, (1998).

144. C. Lavedan, E. Leroy, A. Dehejia, S. Buchholtz, A. Dutra, R. L. Nussbaum, M. H. Polymeropoulos, Identification, localization and characterization of the human gammasynuclein gene. Human genetics 103(1):106-112, (1998).

145. B. R. Lee, T. Kamitani, Improved immunodetection of endogenous alpha-synuclein. PloS one 6(8):e23939, (2011).

146. G. W. Lee, F. Melchior, M. J. Matunis, R. Mahajan, Q. Tian, P. Anderson, Modification of Ran GTPase-activating protein by the small ubiquitin-related modifier SUMO-1 requires Ubc9, an E2-type ubiquitin-conjugating enzyme homologue. The Journal of biological chemistry 273(11):6503-6507, (1998).

147. H. J. Lee, E. J. Bae, S. J. Lee, Extracellular alpha--synuclein-a novel and crucial factor in Lewy body diseases. Nat Rev Neurol 10(2):92-98, (2014).

148. H. J. Lee, S. Patel, S. J. Lee, Intravesicular localization and exocytosis of alpha-synuclein and its aggregates. The Journal of neuroscience : the official journal of the Society for Neuroscience 25(25):6016-6024, (2005). 
149. V. M. Lee, J. Q. Trojanowski, Mechanisms of Parkinson's disease linked to pathological alpha-synuclein: new targets for drug discovery. Neuron 52(1):33-38, (2006).

150. E. Leroy, R. Boyer, G. Auburger, B. Leube, G. Ulm, E. Mezey, G. Harta, M. J. Brownstein, S. Jonnalagada, T. Chernova, A. Dehejia, C. Lavedan, T. Gasser, P. J. Steinbach, K. D. Wilkinson, M. H. Polymeropoulos, The ubiquitin pathway in Parkinson's disease. Nature 395(6701):451-452, (1998).

151. J. Y. Li, E. Englund, J. L. Holton, D. Soulet, P. Hagell, A. J. Lees, T. Lashley, N. P. Quinn, S. Rehncrona, A. Bjorklund, H. Widner, T. Revesz, O. Lindvall, P. Brundin, Lewy bodies in grafted neurons in subjects with Parkinson's disease suggest host-to-graft disease propagation. Nature medicine 14(5):501-503, (2008).

152. Y. Li, H. Wang, S. Wang, D. Quon, Y. W. Liu, B. Cordell, Positive and negative regulation of APP amyloidogenesis by sumoylation. Proceedings of the National Academy of Sciences of the United States of America 100(1):259-264, (2003).

153. Y. Liu, M. Qiang, Y. Wei, R. He, A novel molecular mechanism for nitrated \{alpha\}-synucleininduced cell death. Journal of molecular cell biology 3(4):239-249, (2011).

154. L. M. Lois, C. D. Lima, Structures of the SUMO E1 provide mechanistic insights into SUMO activation and E2 recruitment to E1. 24(3):439-451, (2005).

155. V. Luga, L. Zhang, A. M. Viloria-Petit, A. A. Ogunjimi, M. R. Inanlou, E. Chiu, M. Buchanan, A. N. Hosein, M. Basik, J. L. Wrana, Exosomes mediate stromal mobilization of autocrine Wnt-PCP signaling in breast cancer cell migration. Cell 151(7):1542-1556, (2012).

156. K. C. Luk, V. Kehm, J. Carroll, B. Zhang, P. O'Brien, J. Q. Trojanowski, V. M. Lee, Pathological alpha-synuclein transmission initiates Parkinson-like neurodegeneration in nontransgenic mice. Science 338(6109):949-953, (2012a).

157. K. C. Luk, V. M. Kehm, B. Zhang, P. O'Brien, J. Q. Trojanowski, V. M. Lee, Intracerebral inoculation of pathological alpha-synuclein initiates a rapidly progressive neurodegenerative alpha-synucleinopathy in mice. The Journal of experimental medicine 209(5):975-986, (2012b).

158. K. C. Luk, V. M. Lee, Modeling Lewy pathology propagation in Parkinson's disease. Parkinsonism \& related disorders 20 Suppl 1(S85-87, (2014).

159. H. B. Luo, Y. Y. Xia, X. J. Shu, Z. C. Liu, Y. Feng, X. H. Liu, G. Yu, G. Yin, Y. S. Xiong, K. Zeng, J. Jiang, K. Q. Ye, X. C. Wang, J. Z. Wang, SUMOylation at K340 inhibits tau degradation through deregulating its phosphorylation and ubiquitination. Proceedings of the National Academy of Sciences of the United States of America 111(46):16586-16591, (2014).

160. J. P. Luzio, S. R. Gray, N. A. Bright, Endosome-lysosome fusion. Biochemical Society transactions 38(6): 1413-1416, (2010).

161. L. Maroteaux, J. T. Campanelli, R. H. Scheller, Synuclein: a neuron-specific protein localized to the nucleus and presynaptic nerve terminal. The Journal of neuroscience : the official journal of the Society for Neuroscience 8(8):2804-2815, (1988).

162. L. Maroteaux, R. H. Scheller, The rat brain synucleins; family of proteins transiently associated with neuronal membrane. Brain research. Molecular brain research 11(3-4):335343, (1991).

163. S. Martin, K. A. Wilkinson, A. Nishimune, J. M. Henley, Emerging extranuclear roles of protein SUMOylation in neuronal function and dysfunction. Nature reviews. Neuroscience 8(12):948-959, (2007).

164. I. F. Mata, W. J. Wedemeyer, M. J. Farrer, J. P. Taylor, K. A. Gallo, LRRK2 in Parkinson's disease: protein domains and functional insights. Trends in neurosciences 29(5):286-293, (2006).

165. S. Mathivanan, H. Ji, B. J. Tauro, Y. S. Chen, R. J. Simpson, Identifying mutated proteins secreted by colon cancer cell lines using mass spectrometry. Journal of proteomics 76 Spec No.(141-149, (2012). 
166. I. G. McKeith, D. W. Dickson, J. Lowe, M. Emre, J. T. O'Brien, H. Feldman, J. Cummings, J. E. Duda, C. Lippa, E. K. Perry, D. Aarsland, H. Arai, C. G. Ballard, B. Boeve, D. J. Burn, D. Costa, T. Del Ser, B. Dubois, D. Galasko, S. Gauthier, C. G. Goetz, E. GomezTortosa, G. Halliday, L. A. Hansen, J. Hardy, T. Iwatsubo, R. N. Kalaria, D. Kaufer, R. A. Kenny, A. Korczyn, K. Kosaka, V. M. Lee, A. Lees, I. Litvan, E. Londos, O. L. Lopez, S. Minoshima, Y. Mizuno, J. A. Molina, E. B. Mukaetova-Ladinska, F. Pasquier, R. H. Perry, J. B. Schulz, J. Q. Trojanowski, M. Yamada, Diagnosis and management of dementia with Lewy bodies: third report of the DLB Consortium. Neurology 65(12):1863-1872, (2005).

167. I. G. McKeith, E. Rowan, K. Askew, A. Naidu, L. Allan, N. Barnett, D. Lett, U. P. Mosimann, D. Burn, J. T. O'Brien, More severe functional impairment in dementia with lewy bodies than Alzheimer disease is related to extrapyramidal motor dysfunction. The American journal of geriatric psychiatry : official journal of the American Association for Geriatric Psychiatry 14(7):582-588, (2006).

168. P. J. McLean, S. Ribich, B. T. Hyman, Subcellular localization of alpha-synuclein in primary neuronal cultures: effect of missense mutations. Journal of neural transmission. Supplementum 58):53-63, (2000).

169. F. Melchior, SUMO--nonclassical ubiquitin. Annual review of cell and developmental biology 16(591-626, (2000).

170. I. Mellman, Endocytosis and molecular sorting. Annual review of cell and developmental biology 12(575-625, (1996).

171. I. Mendez, A. Vinuela, A. Astradsson, K. Mukhida, P. Hallett, H. Robertson, T. Tierney, R. Holness, A. Dagher, J. Q. Trojanowski, O. Isacson, Dopamine neurons implanted into people with Parkinson's disease survive without pathology for 14 years. Nature medicine 14(5):507-509, (2008).

172. E. Meulmeester, M. Kunze, H. H. Hsiao, H. Urlaub, F. Melchior, Mechanism and consequences for paralog-specific sumoylation of ubiquitin-specific protease 25. Molecular cell 30(5):610-619, (2008).

173. A. Michael, S. D. Bajracharya, P. S. Yuen, H. Zhou, R. A. Star, G. G. Illei, I. Alevizos, Exosomes from human saliva as a source of microRNA biomarkers. Oral diseases 16(1):3438, (2010).

174. A. Minty, X. Dumont, M. Kaghad, D. Caput, Covalent modification of p73alpha by SUMO-1. Two-hybrid screening with p73 identifies novel SUMO-1-interacting proteins and a SUMO-1 interaction motif. The Journal of biological chemistry 275(46):36316-36323, (2000).

175. S. Misra, J. H. Hurley, Crystal structure of a phosphatidylinositol 3-phosphate-specific membrane-targeting motif, the FYVE domain of Vps27p. Cell 97(5):657-666, (1999).

176. F. Mori, K. Tanji, M. Yoshimoto, H. Takahashi, K. Wakabayashi, Immunohistochemical comparison of alpha- and beta-synuclein in adult rat central nervous system. Brain research 941(1-2):118-126, (2002).

177. H. R. Morris, J. R. Vaughan, S. R. Datta, R. Bandopadhyay, H. A. Rohan De Silva, A. Schrag, N. J. Cairns, D. Burn, U. Nath, P. L. Lantos, S. Daniel, A. J. Lees, N. P. Quinn, N. W. Wood, Multiple system atrophy/progressive supranuclear palsy: alpha-Synuclein, synphilin, tau, and APOE. Neurology 55(12):1918-1920, (2000).

178. A. L. Mougenot, S. Nicot, A. Bencsik, E. Morignat, J. Verchere, L. Lakhdar, S. Legastelois, T. Baron, Prion-like acceleration of a synucleinopathy in a transgenic mouse model. Neurobiology of aging 33(9):2225-2228, (2012).

179. D. Mukhopadhyay, M. Dasso, Modification in reverse: the SUMO proteases. Trends in biochemical sciences 32(6):286-295, (2007).

180. I. V. Murray, B. I. Giasson, S. M. Quinn, V. Koppaka, P. H. Axelsen, H. Ischiropoulos, J. Q. Trojanowski, V. M. Lee, Role of alpha-synuclein carboxy-terminus on fibril formation in vitro. Biochemistry 42(28):8530-8540, (2003).

181. S. Nakajo, S. Shioda, Y. Nakai, K. Nakaya, Localization of phosphoneuroprotein 14 (PNP 14) and its mRNA expression in rat brain determined by immunocytochemistry and in situ hybridization. Brain research. Molecular brain research 27(1):81-86, (1994). 
182. T. Nakamura, H. Yamashita, T. Takahashi, S. Nakamura, Activated Fyn phosphorylates alpha-synuclein at tyrosine residue 125 . Biochemical and biophysical research communications 280(4):1085-1092, (2001).

183. V. M. Nemani, W. Lu, V. Berge, K. Nakamura, B. Onoa, M. K. Lee, F. A. Chaudhry, R. A. Nicoll, R. H. Edwards, Increased expression of alpha-synuclein reduces neurotransmitter release by inhibiting synaptic vesicle reclustering after endocytosis. Neuron 65(1):66-79, (2010).

184. M. Okochi, J. Walter, A. Koyama, S. Nakajo, M. Baba, T. Iwatsubo, L. Meijer, P. J. Kahle, C. Haass, Constitutive phosphorylation of the Parkinson's disease associated alphasynuclein. The Journal of biological chemistry 275(1):390-397, (2000).

185. C. W. Olanow, C. G. Goetz, J. H. Kordower, A. J. StoessI, V. Sossi, M. F. Brin, K. M. Shannon, G. M. Nauert, D. P. Perl, J. Godbold, T. B. Freeman, A double-blind controlled trial of bilateral fetal nigral transplantation in Parkinson's disease. Annals of neurology 54(3):403-414, (2003).

186. M. Ostrowski, N. B. Carmo, S. Krumeich, I. Fanget, G. Raposo, A. Savina, C. F. Moita, K. Schauer, A. N. Hume, R. P. Freitas, B. Goud, P. Benaroch, N. Hacohen, M. Fukuda, C. Desnos, M. C. Seabra, F. Darchen, S. Amigorena, L. F. Moita, C. Thery, Rab27a and Rab27b control different steps of the exosome secretion pathway. Nature cell biology 12(1):19-30; sup pp 11-13, (2010).

187. T. F. Outeiro, S. Lindquist, Yeast cells provide insight into alpha-synuclein biology and pathobiology. Science 302(5651):1772-1775, (2003).

188. T. F. Outeiro, P. Putcha, J. E. Tetzlaff, R. Spoelgen, M. Koker, F. Carvalho, B. T. Hyman, P. J. McLean, Formation of toxic oligomeric alpha-synuclein species in living cells. PloS one 3(4):e1867, (2008).

189. D. Owerbach, E. M. McKay, E. T. Yeh, K. H. Gabbay, K. M. Bohren, A proline-90 residue unique to SUMO-4 prevents maturation and sumoylation. Biochemical and biophysical research communications 337(2):517-520, (2005).

190. T. Ozawa, H. Takano, O. Onodera, H. Kobayashi, T. Ikeuchi, R. Koide, K. Okuizumi, T. Shimohata, K. Wakabayashi, H. Takahashi, S. Tsuji, No mutation in the entire coding region of the alpha-synuclein gene in pathologically confirmed cases of multiple system atrophy. Neuroscience letters 270(2):110-112, (1999).

191. J. J. Palvimo, PIAS proteins as regulators of small ubiquitin-related modifier (SUMO) modifications and transcription. Biochemical Society transactions 35(Pt 6):1405-1408, (2007).

192. B. T. Pan, R. M. Johnstone, Fate of the transferrin receptor during maturation of sheep reticulocytes in vitro: selective externalization of the receptor. Cell 33(3):967-978, (1983).

193. B. T. Pan, K. Teng, C. Wu, M. Adam, R. M. Johnstone, Electron microscopic evidence for externalization of the transferrin receptor in vesicular form in sheep reticulocytes. The Journal of cell biology 101(3):942-948, (1985).

194. K. H. Park, B. J. Kim, J. Kang, T. S. Nam, J. M. Lim, H. T. Kim, J. K. Park, Y. G. Kim, S. W. Chae, U. H. Kim, Ca2+ signaling tools acquired from prostasomes are required for progesterone-induced sperm motility. Science signaling 4(173):ra31, (2011).

195. I. Parolini, C. Federici, C. Raggi, L. Lugini, S. Palleschi, A. De Milito, C. Coscia, E. lessi, M. Logozzi, A. Molinari, M. Colone, M. Tatti, M. Sargiacomo, S. Fais, Microenvironmental $\mathrm{pH}$ is a key factor for exosome traffic in tumor cells. The Journal of biological chemistry 284(49):34211-34222, (2009).

196. D. M. Pegtel, K. Cosmopoulos, D. A. Thorley-Lawson, M. A. van Eijndhoven, E. S. Hopmans, J. L. Lindenberg, T. D. de Gruijl, T. Wurdinger, J. M. Middeldorp, Functional delivery of viral miRNAs via exosomes. Proceedings of the National Academy of Sciences of the United States of America 107(14):6328-6333, (2010).

197. H. R. B. Pelham, SNAREs and the specificity of membrane fusion. Trends in cell biology 11(3):99-101, (2001).

198. R. J. Perrin, W. S. Woods, D. F. Clayton, J. M. George, Interaction of human alphaSynuclein and Parkinson's disease variants with phospholipids. Structural analysis using sitedirected mutagenesis. The Journal of biological chemistry 275(44):34393-34398, (2000). 
199. A. Pichler, A. Gast, J. S. Seeler, A. Dejean, F. Melchior, The nucleoporin RanBP2 has SUMO1 E3 ligase activity. Cell 108(1):109-120, (2002).

200. R. C. Piper, P. J. Lehner, Endosomal transport via ubiquitination. Trends in cell biology 21(11):647-655, (2011).

201. T. Pisitkun, R. F. Shen, M. A. Knepper, Identification and proteomic profiling of exosomes in human urine. Proceedings of the National Academy of Sciences of the United States of America 101(36):13368-13373, (2004).

202. M. Pitschke, R. Prior, M. Haupt, D. Riesner, Detection of single amyloid beta-protein aggregates in the cerebrospinal fluid of Alzheimer's patients by fluorescence correlation spectroscopy. Nature medicine 4(7):832-834, (1998).

203. E. Pokrishevsky, L. I. Grad, M. Yousefi, J. Wang, I. R. Mackenzie, N. R. Cashman, Aberrant localization of FUS and TDP43 is associated with misfolding of SOD1 in amyotrophic lateral sclerosis. PloS one 7(4):e35050, (2012).

204. M. H. Polymeropoulos, C. Lavedan, E. Leroy, S. E. Ide, A. Dehejia, A. Dutra, B. Pike, H. Root, J. Rubenstein, R. Boyer, E. S. Stenroos, S. Chandrasekharappa, A. Athanassiadou, T. Papapetropoulos, W. G. Johnson, A. M. Lazzarini, R. C. Duvoisin, G. Di lorio, L. I. Golbe, R. L. Nussbaum, Mutation in the alpha-synuclein gene identified in families with Parkinson's disease. Science 276(5321):2045-2047, (1997).

205. K. Post, M. Pitschke, O. Schafer, H. Wille, T. R. Appel, D. Kirsch, I. Mehlhorn, H. Serban, S. B. Prusiner, D. Riesner, Rapid acquisition of beta-sheet structure in the prion protein prior to multimer formation. Biological chemistry 379(11):1307-1317, (1998).

206. D. L. Pountney, F. Chegini, X. Shen, P. C. Blumbergs, W. P. Gai, SUMO-1 marks subdomains within glial cytoplasmic inclusions of multiple system atrophy. Neuroscience letters 381(1-2):74-79, (2005).

207. P. Putcha, K. M. Danzer, L. R. Kranich, A. Scott, M. Silinski, S. Mabbett, C. D. Hicks, J. M. Veal, P. M. Steed, B. T. Hyman, P. J. McLean, Brain-permeable small-molecule inhibitors of Hsp90 prevent alpha-synuclein oligomer formation and rescue alpha-synuclein-induced toxicity. The Journal of pharmacology and experimental therapeutics 332(3):849-857, (2010).

208. G. Rabinowits, C. Gercel-Taylor, J. M. Day, D. D. Taylor, G. H. Kloecker, Exosomal microRNA: a diagnostic marker for lung cancer. Clinical lung cancer 10(1):42-46, (2009).

209. C. Raiborg, K. G. Bache, A. Mehlum, E. Stang, H. Stenmark, Hrs recruits clathrin to early endosomes. EMBO J 20(17):5008-5021, (2001).

210. C. Raiborg, T. E. Rusten, H. Stenmark, Protein sorting into multivesicular endosomes. Current opinion in cell biology 15(4):446-455, (2003).

211. L. Rajendran, M. Honsho, T. R. Zahn, P. Keller, K. D. Geiger, P. Verkade, K. Simons, Alzheimer's disease beta-amyloid peptides are released in association with exosomes. Proceedings of the National Academy of Sciences of the United States of America 103(30):11172-11177, (2006).

212. S. Rana, S. Yue, D. Stadel, M. Zoller, Toward tailored exosomes: the exosomal tetraspanin web contributes to target cell selection. The international journal of biochemistry \& cell biology 44(9):1574-1584, (2012).

213. S. Rana, M. Zoller, Exosome target cell selection and the importance of exosomal tetraspanins: a hypothesis. Biochemical Society transactions 39(2):559-562, (2011).

214. G. Raposo, H. W. Nijman, W. Stoorvogel, R. Liejendekker, C. V. Harding, C. J. Melief, H. J. Geuze, B lymphocytes secrete antigen-presenting vesicles. The Journal of experimental medicine 183(3):1161-1172, (1996).

215. G. Raposo, W. Stoorvogel, Extracellular vesicles: exosomes, microvesicles, and friends. The Journal of cell biology 200(4):373-383, (2013).

216. J. Ratajczak, M. Wysoczynski, F. Hayek, A. Janowska-Wieczorek, M. Z. Ratajczak, Membrane-derived microvesicles: important and underappreciated mediators of cell-to-cell communication. Leukemia 20(9):1487-1495, (2006). 
217. A. Recasens, B. Dehay, J. Bove, I. Carballo-Carbajal, S. Dovero, A. Perez-Villalba, P. O. Fernagut, J. Blesa, A. Parent, C. Perier, I. Farinas, J. A. Obeso, E. Bezard, M. Vila, Lewy body extracts from Parkinson disease brains trigger alpha-synuclein pathology and neurodegeneration in mice and monkeys. Annals of neurology 75(3):351-362, (2014).

218. I. Remy, S. W. Michnick, A highly sensitive protein-protein interaction assay based on Gaussia luciferase. Nature methods 3(12):977-979, (2006).

219. D. Reverter, C. D. Lima, Insights into E3 ligase activity revealed by a SUMO-RanGAP1Ubc9-Nup358 complex. Nature 435(7042):687-692, (2005).

220. N. L. Rey, G. H. Petit, L. Bousset, R. Melki, P. Brundin, Transfer of human alpha-synuclein from the olfactory bulb to interconnected brain regions in mice. Acta neuropathologica 126(4):555-573, (2013).

221. C. Robertson, S. A. Booth, D. R. Beniac, M. B. Coulthart, T. F. Booth, A. McNicol, Cellular prion protein is released on exosomes from activated platelets. Blood 107(10):3907-3911, (2006).

222. M. S. Rodriguez, C. Dargemont, R. T. Hay, SUMO-1 conjugation in vivo requires both a consensus modification motif and nuclear targeting. The Journal of biological chemistry 276(16):12654-12659, (2001).

223. G. Rosas-Acosta, W. K. Russell, A. Deyrieux, D. H. Russell, V. G. Wilson, A universal strategy for proteomic studies of SUMO and other ubiquitin-like modifiers. Molecular \& cellular proteomics : MCP 4(1):56-72, (2005).

224. I. Roxrud, H. Stenmark, L. Malerod, ESCRT \& Co. Biology of the cell / under the auspices of the European Cell Biology Organization 102(5):293-318, (2010).

225. H. Saitoh, J. Hinchey, Functional heterogeneity of small ubiquitin-related protein modifiers SUMO-1 versus SUMO-2/3. The Journal of biological chemistry 275(9):6252-6258, (2000).

226. J. Sambrook, D. W. Russell, Molecular cloning: a laboratory manual (3-volume set). (Cold spring harbor laboratory press Cold Spring Harbor, New York:, 2001), vol. 999.

227. D. A. Sampson, M. Wang, M. J. Matunis, The small ubiquitin-like modifier-1 (SUMO-1) consensus sequence mediates Ubc9 binding and is essential for SUMO-1 modification. The Journal of biological chemistry 276(24):21664-21669, (2001).

228. A. Sapetschnig, G. Rischitor, H. Braun, A. Doll, M. Schergaut, F. Melchior, G. Suske, Transcription factor Sp3 is silenced through SUMO modification by PIAS1. The EMBO journal 21(19):5206-5215, (2002).

229. J. S. Schorey, S. Bhatnagar, Exosome function: from tumor immunology to pathogen biology. Traffic 9(6):871-881, (2008).

230. D. Schubert, S. Humphreys, C. Baroni, M. Cohn, In vitro differentiation of a mouse neuroblastoma. Proceedings of the National Academy of Sciences of the United States of America 64(1):316-323, (1969).

231. S. Schulz, G. Chachami, L. Kozaczkiewicz, U. Winter, N. Stankovic-Valentin, P. Haas, K. Hofmann, H. Urlaub, H. Ovaa, J. Wittbrodt, E. Meulmeester, F. Melchior, Ubiquitin-specific protease-like 1 (USPL1) is a SUMO isopeptidase with essential, non-catalytic functions. EMBO reports 13(10):930-938, (2012).

232. P. Schwille, J. Bieschke, F. Oehlenschlager, Kinetic investigations by fluorescence correlation spectroscopy: the analytical and diagnostic potential of diffusion studies. Biophysical chemistry 66(2-3):211-228, (1997).

233. D. Scott, S. Roy, alpha-Synuclein inhibits intersynaptic vesicle mobility and maintains recycling-pool homeostasis. The Journal of neuroscience : the official journal of the Society for Neuroscience 32(30):10129-10135, (2012).

234. H. Sheldon, E. Heikamp, H. Turley, R. Dragovic, P. Thomas, C. E. Oon, R. Leek, M. Edelmann, B. Kessler, R. C. Sainson, I. Sargent, J. L. Li, A. L. Harris, New mechanism for Notch signaling to endothelium at a distance by Delta-like 4 incorporation into exosomes. Blood 116(13):2385-2394, (2010).

235. B. Shen, Y. Fang, N. Wu, S. J. Gould, Biogenesis of the Posterior Pole Is Mediated by the Exosome/Microvesicle Protein-sorting Pathway. J Biol Chem 286(51):44162-44176, (2011a). 
236. B. Shen, N. Wu, J. M. Yang, S. J. Gould, Protein targeting to exosomes/microvesicles by plasma membrane anchors. The Journal of biological chemistry 286(16):14383-14395, (2011b).

237. S. B. Shields, R. C. Piper, How ubiquitin functions with ESCRTs. Traffic 12(10):1306-1317, (2011).

238. E. J. Shin, H. M. Shin, E. Nam, W. S. Kim, J. H. Kim, B. H. Oh, Y. Yun, DeSUMOylating isopeptidase: a second class of SUMO protease. EMBO reports 13(4):339-346, (2012).

239. Y. Shinbo, T. Niki, T. Taira, H. Ooe, K. Takahashi-Niki, C. Maita, C. Seino, S. M. IguchiAriga, H. Ariga, Proper SUMO-1 conjugation is essential to DJ-1 to exert its full activities. Cell death and differentiation 13(1):96-108, (2006).

240. V. V. Shvadchak, L. J. Falomir-Lockhart, D. A. Yushchenko, T. M. Jovin, Specificity and kinetics of alpha-synuclein binding to model membranes determined with fluorescent excited state intramolecular proton transfer (ESIPT) probe. The Journal of biological chemistry 286(15):13023-13032, (2011).

241. M. Simons, G. Raposo, Exosomes--vesicular carriers for intercellular communication. Current opinion in cell biology 21(4):575-581, (2009).

242. J. Song, L. K. Durrin, T. A. Wilkinson, T. G. Krontiris, Y. Chen, Identification of a SUMObinding motif that recognizes SUMO-modified proteins. Proceedings of the National Academy of Sciences of the United States of America 101(40):14373-14378, (2004).

243. Y. J. Song, D. M. Lundvig, Y. Huang, W. P. Gai, P. C. Blumbergs, P. Hojrup, D. Otzen, G. M. Halliday, P. H. Jensen, p25alpha relocalizes in oligodendroglia from myelin to cytoplasmic inclusions in multiple system atrophy. The American journal of pathology 171(4):1291-1303, (2007).

244. M. G. Spillantini, R. A. Crowther, R. Jakes, N. J. Cairns, P. L. Lantos, M. Goedert, Filamentous alpha-synuclein inclusions link multiple system atrophy with Parkinson's disease and dementia with Lewy bodies. Neuroscience letters 251(3):205-208, (1998a).

245. M. G. Spillantini, R. A. Crowther, R. Jakes, M. Hasegawa, M. Goedert, alpha-Synuclein in filamentous inclusions of Lewy bodies from Parkinson's disease and dementia with lewy bodies. Proceedings of the National Academy of Sciences of the United States of America 95(11):6469-6473, (1998b).

246. M. G. Spillantini, M. L. Schmidt, V. M. Lee, J. Q. Trojanowski, R. Jakes, M. Goedert, Alpha-synuclein in Lewy bodies. Nature 388(6645):839-840, (1997).

247. J. S. Steffan, N. Agrawal, J. Pallos, E. Rockabrand, L. C. Trotman, N. Slepko, K. Illes, T. Lukacsovich, Y. Z. Zhu, E. Cattaneo, P. P. Pandolfi, L. M. Thompson, J. L. Marsh, SUMO modification of Huntingtin and Huntington's disease pathology. Science 304(5667):100-104, (2004).

248. P. Stehmeier, S. Müller, Phospho-regulated SUMO interaction modules connect the SUMO system to CK2 signaling. Molecular cell 33(3):400-409, (2009).

249. H. Stenmark, R. G. Parton, O. Steele-Mortimer, A. Lutcke, J. Gruenberg, M. Zerial, Inhibition of rab5 GTPase activity stimulates membrane fusion in endocytosis. EMBO J 13(6):1287-1296, (1994a).

250. H. Stenmark, A. Valencia, O. Martinez, O. Ullrich, B. Goud, M. Zerial, Distinct structural elements of rab5 define its functional specificity. EMBO J 13(3):575-583, (1994b).

251. J. Storch, A. M. Kleinfeld, Transfer of long-chain fluorescent free fatty acids between unilamellar vesicles. Biochemistry 25(7):1717-1726, (1986).

252. K. Strauss, C. Goebel, H. Runz, W. Möbius, S. Weiss, I. Feussner, M. Simons, A. Schneider, Exosome secretion ameliorates lysosomal storage of cholesterol in Niemann-Pick type C disease. The Journal of biological chemistry 285(34):26279-26288, (2010).

253. C. Subra, K. Laulagnier, B. Perret, M. Record, Exosome lipidomics unravels lipid sorting at the level of multivesicular bodies. Biochimie 89(2):205-212, (2007).

254. H. Sun, J. D. Leverson, T. Hunter, Conserved function of RNF4 family proteins in eukaryotes: targeting a ubiquitin ligase to SUMOylated proteins. The EMBO journal 26(18):4102-4112, (2007). 
255. T. Taira, Y. Saito, T. Niki, S. M. Iguchi-Ariga, K. Takahashi, H. Ariga, DJ-1 has a role in antioxidative stress to prevent cell death. EMBO reports 5(2):213-218, (2004).

256. M. Takahashi, H. Miyata, F. Kametani, T. Nonaka, H. Akiyama, S. I. Hisanaga, M. Hasegawa, Extracellular association of APP and tau fibrils induces intracellular aggregate formation of tau. Acta neuropathologica (2015).

257. Y. Takahashi, T. Kahyo, E. A. Toh, H. Yasuda, Y. Kikuchi, Yeast Ull1/Siz1 is a novel SUMO1/Smt3 ligase for septin components and functions as an adaptor between conjugating enzyme and substrates. The Journal of biological chemistry 276(52):48973-48977, (2001).

258. M. H. Tatham, E. Jaffray, O. A. Vaughan, J. M. Desterro, C. H. Botting, J. H. Naismith, R. T. Hay, Polymeric chains of SUMO-2 and SUMO-3 are conjugated to protein substrates by SAE1/SAE2 and Ubc9. The Journal of biological chemistry 276(38):35368-35374, (2001).

259. B. J. Tauro, D. W. Greening, R. A. Mathias, S. Mathivanan, H. Ji, R. J. Simpson, Two distinct populations of exosomes are released from LIM1863 colon carcinoma cell-derived organoids. Molecular \& cellular proteomics : MCP 12(3):587-598, (2013).

260. S. Tenreiro, K. Eckermann, T. F. Outeiro, Protein phosphorylation in neurodegeneration: friend or foe? Frontiers in molecular neuroscience 7(42, (2014).

261. J. E. Tetzlaff, P. Putcha, T. F. Outeiro, A. Ivanov, O. Berezovska, B. T. Hyman, P. J. McLean, CHIP targets toxic alpha-Synuclein oligomers for degradation. The Journal of biological chemistry 283(26):17962-17968, (2008).

262. N. Thayanidhi, J. R. Helm, D. C. Nycz, M. Bentley, Y. Liang, J. C. Hay, Alpha-synuclein delays endoplasmic reticulum (ER)-to-Golgi transport in mammalian cells by antagonizing ER/Golgi SNAREs. Molecular biology of the cell 21(11):1850-1863, (2010).

263. A. C. Theos, S. T. Truschel, D. Tenza, I. Hurbain, D. C. Harper, J. F. Berson, P. C. Thomas, G. Raposo, M. S. Marks, A lumenal domain-dependent pathway for sorting to intralumenal vesicles of multivesicular endosomes involved in organelle morphogenesis. Developmental cell 10(3):343-354, (2006).

264. C. Thery, S. Amigorena, The cell biology of antigen presentation in dendritic cells. Current opinion in immunology 13(1):45-51, (2001a).

265. C. Théry, S. Amigorena, G. Raposo, A. Clayton, Isolation and characterization of exosomes from cell culture supernatants and biological fluids. Current protocols in cell biology / editorial board, Juan S. Bonifacino ... [et al.] Chapter 3(Unit 3 22, (2006).

266. C. Thery, M. Boussac, P. Veron, P. Ricciardi-Castagnoli, G. Raposo, J. Garin, S. Amigorena, Proteomic analysis of dendritic cell-derived exosomes: a secreted subcellular compartment distinct from apoptotic vesicles. J Immunol 166(12):7309-7318, (2001b).

267. C. Théry, M. Ostrowski, E. Segura, Membrane vesicles as conveyors of immune responses. Nature reviews. Immunology 9(8):581-593, (2009).

268. T. Tokuda, M. M. Qureshi, M. T. Ardah, S. Varghese, S. A. Shehab, T. Kasai, N. Ishigami, A. Tamaoka, M. Nakagawa, O. M. El-Agnaf, Detection of elevated levels of alpha-synuclein oligomers in CSF from patients with Parkinson disease. Neurology 75(20):1766-1772, (2010).

269. H. Towbin, T. Staehelin, J. Gordon, Electrophoretic transfer of proteins from polyacrylamide gels to nitrocellulose sheets: procedure and some applications. Proceedings of the National Academy of Sciences of the United States of America 76(9):4350-4354, (1979).

270. K. Trajkovic, C. Hsu, S. Chiantia, L. Rajendran, D. Wenzel, F. Wieland, P. Schwille, B. Brugger, M. Simons, Ceramide triggers budding of exosome vesicles into multivesicular endosomes. Science 319(5867):1244-1247, (2008).

271. P. H. Tu, J. E. Galvin, M. Baba, B. Giasson, T. Tomita, S. Leight, S. Nakajo, T. Iwatsubo, J. Q. Trojanowski, V. M. Lee, Glial cytoplasmic inclusions in white matter oligodendrocytes of multiple system atrophy brains contain insoluble alpha-synuclein. Annals of neurology 44(3):415-422, (1998).

272. Y. Uchimura, M. Nakamura, K. Sugasawa, M. Nakao, H. Saitoh, Overproduction of eukaryotic SUMO-1- and SUMO-2-conjugated proteins in Escherichia coli. Analytical biochemistry 331(1):204-206, (2004). 
273. K. Ueda, H. Fukushima, E. Masliah, Y. Xia, A. Iwai, M. Yoshimoto, D. A. Otero, J. Kondo, Y. Ihara, T. Saitoh, Molecular cloning of cDNA encoding an unrecognized component of amyloid in Alzheimer disease. Proceedings of the National Academy of Sciences of the United States of America 90(23):11282-11286, (1993).

274. H. D. Ulrich, SUMO modification: wrestling with protein conformation. Current biology : $C B$ 15(7):R257-259, (2005).

275. A. Ulusoy, R. E. Musgrove, R. Rusconi, M. Klinkenberg, M. Helwig, A. Schneider, D. A. Di Monte, Neuron-to-neuron alpha-synuclein propagation in vivo is independent of neuronal injury. Acta neuropathologica communications 3(1):13, (2015).

276. A. Ulusoy, R. Rusconi, B. I. Perez-Revuelta, R. E. Musgrove, M. Helwig, B. WinzenReichert, D. A. Di Monte, Caudo-rostral brain spreading of alpha-synuclein through vagal connections. EMBO molecular medicine 5(7):1051-1059, (2013).

277. J. W. Um, K. C. Chung, Functional modulation of parkin through physical interaction with SUMO-1. Journal of neuroscience research 84(7):1543-1554, (2006).

278. V. N. Uversky, Neuropathology, biochemistry, and biophysics of alpha-synuclein aggregation. Journal of neurochemistry 103(1):17-37, (2007).

279. V. N. Uversky, J. Li, P. Souillac, I. S. Millett, S. Doniach, R. Jakes, M. Goedert, A. L. Fink, Biophysical properties of the synucleins and their propensities to fibrillate: inhibition of alphasynuclein assembly by beta- and gamma-synucleins. The Journal of biological chemistry 277(14):11970-11978, (2002).

280. H. Valadi, K. Ekstrom, A. Bossios, M. Sjostrand, J. J. Lee, J. O. Lotvall, Exosomemediated transfer of mRNAs and microRNAs is a novel mechanism of genetic exchange between cells. Nature cell biology 9(6):654-659, (2007).

281. E. M. Valente, F. Brancati, V. Caputo, E. A. Graham, M. B. Davis, A. Ferraris, M. M. Breteler, T. Gasser, V. Bonifati, A. R. Bentivoglio, G. De Michele, A. Durr, P. Cortelli, A. Filla, G. Meco, B. A. Oostra, A. Brice, A. Albanese, B. Dallapiccola, N. W. Wood, PARK6 is a common cause of familial parkinsonism. Neurological sciences : official journal of the Italian Neurological Society and of the Italian Society of Clinical Neurophysiology 23 Suppl 2(S117-118, (2002a).

282. E. M. Valente, F. Brancati, A. Ferraris, E. A. Graham, M. B. Davis, M. M. Breteler, T. Gasser, V. Bonifati, A. R. Bentivoglio, G. De Michele, A. Durr, P. Cortelli, D. Wassilowsky, B. S. Harhangi, N. Rawal, V. Caputo, A. Filla, G. Meco, B. A. Oostra, A. Brice, A. Albanese, B. Dallapiccola, N. W. Wood, PARK6-linked parkinsonism occurs in several European families. Annals of neurology 51(1):14-18, (2002b).

283. A. J. van der Eb, Intermediates in type 5 adenovirus DNA replication. Virology 51(1):11-23, (1973).

284. T. J. van Ham, K. L. Thijssen, R. Breitling, R. M. Hofstra, R. H. Plasterk, E. A. Nollen, C. elegans model identifies genetic modifiers of alpha-synuclein inclusion formation during aging. PLoS genetics 4(3):e1000027, (2008).

285. G. van Meer, D. R. Voelker, G. W. Feigenson, Membrane lipids: where they are and how they behave. Nature reviews. Molecular cell biology 9(2):112-124, (2008).

286. G. van Niel, S. Charrin, S. Simoes, M. Romao, L. Rochin, P. Saftig, M. S. Marks, E. Rubinstein, G. Raposo, The tetraspanin CD63 regulates ESCRT-independent and dependent endosomal sorting during melanogenesis. Developmental cell 21(4):708-721, (2011).

287. G. van Niel, I. Porto-Carreiro, S. Simoes, G. Raposo, Exosomes: a common pathway for a specialized function. Journal of biochemistry 140(1):13-21, (2006).

288. L. J. Vella, D. L. Greenwood, R. Cappai, J. P. Scheerlinck, A. F. Hill, Enrichment of prion protein in exosomes derived from ovine cerebral spinal fluid. Veterinary immunology and immunopathology 124(3-4):385-393, (2008).

289. L. J. Vella, R. A. Sharples, V. A. Lawson, C. L. Masters, R. Cappai, A. F. Hill, Packaging of prions into exosomes is associated with a novel pathway of PrP processing. The Journal of pathology 211(5):582-590, (2007). 
290. A. C. Vertegaal, J. S. Andersen, S. C. Ogg, R. T. Hay, M. Mann, A. I. Lamond, Distinct and overlapping sets of SUMO-1 and SUMO-2 target proteins revealed by quantitative proteomics. Molecular \& cellular proteomics : MCP 5(12):2298-2310, (2006).

291. C. Villarroya-Beltri, C. Gutierrez-Vazquez, F. Sanchez-Cabo, D. Perez-Hernandez, J. Vazquez, N. Martin-Cofreces, D. J. Martinez-Herrera, A. Pascual-Montano, M. Mittelbrunn, F. Sanchez-Madrid, Sumoylated hnRNPA2B1 controls the sorting of miRNAs into exosomes through binding to specific motifs. Nature communications 4(2980, (2013).

292. V. Vingtdeux, N. Sergeant, L. Buée, Potential contribution of exosomes to the prion-like propagation of lesions in Alzheimer's disease. Frontiers in physiology 3(229, (2012).

293. K. Wakabayashi, M. Yoshimoto, S. Tsuji, H. Takahashi, Alpha-synuclein immunoreactivity in glial cytoplasmic inclusions in multiple system atrophy. Neuroscience letters 249(2-3):180182, (1998).

294. F. O. Walker, Huntington's Disease. Seminars in neurology 27(2):143-150, (2007).

295. J. D. Walker, C. L. Maier, J. S. Pober, Cytomegalovirus-infected human endothelial cells can stimulate allogeneic CD4+ memory $\mathrm{T}$ cells by releasing antigenic exosomes. J Immunol 182(3):1548-1559, (2009).

296. P. Whitley, B. J. Reaves, M. Hashimoto, A. M. Riley, B. V. Potter, G. D. Holman, Identification of mammalian Vps24p as an effector of phosphatidylinositol 3,5-bisphosphatedependent endosome compartmentalization. The Journal of biological chemistry 278(40):38786-38795, (2003).

297. K. A. Wilkinson, Y. Nakamura, J. M. Henley, Targets and consequences of protein SUMOylation in neurons. Brain research reviews 64(1):195-212, (2010).

298. J. Wolfers, A. Lozier, G. Raposo, A. Regnault, C. Thery, C. Masurier, C. Flament, S. Pouzieux, F. Faure, T. Tursz, E. Angevin, S. Amigorena, L. Zitvogel, Tumor-derived exosomes are a source of shared tumor rejection antigens for CTL cross-priming. Nature medicine 7(3):297-303, (2001).

299. S. J. Wood, J. Wypych, S. Steavenson, J. C. Louis, M. Citron, A. L. Biere, alpha-synuclein fibrillogenesis is nucleation-dependent. Implications for the pathogenesis of Parkinson's disease. The Journal of biological chemistry 274(28):19509-19512, (1999).

300. R. Wubbolts, R. S. Leckie, P. T. Veenhuizen, G. Schwarzmann, W. Möbius, J. Hoernschemeyer, J. W. Slot, H. J. Geuze, W. Stoorvogel, Proteomic and biochemical analyses of human B cell-derived exosomes. Potential implications for their function and multivesicular body formation. The Journal of biological chemistry 278(13):10963-10972, (2003).

301. Y. Xia, T. Saitoh, K. Ueda, S. Tanaka, X. Chen, M. Hashimoto, L. Hsu, C. Conrad, M. Sundsmo, M. Yoshimoto, L. Thal, R. Katzman, E. Masliah, Characterization of the human alpha-synuclein gene: Genomic structure, transcription start site, promoter region and polymorphisms. Journal of Alzheimer's disease : JAD 3(5):485-494, (2001).

302. E. T. Yeh, SUMOylation and De-SUMOylation: wrestling with life's processes. The Journal of biological chemistry 284(13):8223-8227, (2009).

303. S. C. L. Yeo, L. H. Xu, J. H. Ren, V. J. Boulton, M. D. Wagle, C. Liu, G. Ren, P. Wong, R. Zahn, P. Sasajala, H. Y. Yang, R. C. Piper, A. L. Munn, Vps20p and Vta1p interact with Vps4p and function in multivesicular body sorting and endosomal transport in Saccharomyces cerevisiae. Journal of cell science 116(19):3957-3970, (2003).

304. M. Yonetani, T. Nonaka, M. Masuda, Y. Inukai, T. Oikawa, S. Hisanaga, M. Hasegawa, Conversion of wild-type alpha-synuclein into mutant-type fibrils and its propagation in the presence of A30P mutant. The Journal of biological chemistry 284(12):7940-7950, (2009).

305. J. J. Zarranz, J. Alegre, J. C. Gomez-Esteban, E. Lezcano, R. Ros, I. Ampuero, L. Vidal, J. Hoenicka, O. Rodriguez, B. Atares, V. Llorens, E. Gomez Tortosa, T. del Ser, D. G. Munoz, J. G. de Yebenes, The new mutation, E46K, of alpha-synuclein causes Parkinson and Lewy body dementia. Annals of neurology 55(2):164-173, (2004).

306. Y. Q. Zhang, K. D. Sarge, Sumoylation of amyloid precursor protein negatively regulates Abeta aggregate levels. Biochemical and biophysical research communications 374(4):673678, (2008). 
307. J. Zhu, S. Zhu, C. M. Guzzo, N. A. Ellis, K. S. Sung, C. Y. Choi, M. J. Matunis, Small ubiquitin-related modifier (SUMO) binding determines substrate recognition and paralogselective SUMO modification. The Journal of biological chemistry 283(43):29405-29415, (2008).

308. L. Zitvogel, A. Regnault, A. Lozier, J. Wolfers, C. Flament, D. Tenza, P. RicciardiCastagnoli, G. Raposo, S. Amigorena, Eradication of established murine tumors using a novel cell-free vaccine: dendritic cell-derived exosomes. Nature medicine 4(5):594-600, (1998).

309. A. Zomer, T. Vendrig, E. S. Hopmans, M. van Eijndhoven, J. M. Middeldorp, D. M. Pegtel, Exosomes: Fit to deliver small RNA. Communicative \& integrative biology 3(5):447-450, (2010). 


\section{Appendix}

Table 13: Summary of NTA measurements

\begin{tabular}{|c|c|c|}
\hline transfected constructs & $\begin{array}{c}\text { mean particle } \\
\text { concentration } \\
\text { adjusted to cell } \\
\text { number }\end{array}$ & SEM \\
\hline Myc-SUMO-2 wt/ $\alpha-S y n$ wt & $2.52 \mathrm{E}+07$ & $1.67 \mathrm{E}+06$ \\
\hline 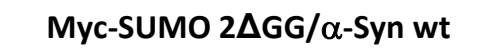 & $2.22 \mathrm{E}+07$ & $2.31 E+06$ \\
\hline $\mathbf{n}$ & 8 & \\
\hline t-test & 0.16738 & \\
\hline$\alpha-S y n$ wt & $2.24 \mathrm{E}+07$ & $2.98 \mathrm{E}+06$ \\
\hline$\alpha$-Syn-SUMO-2 & $2.71 \mathrm{E}+07$ & $4.15 \mathrm{E}+06$ \\
\hline $\mathbf{n}$ & 7 & \\
\hline t-test & 0.06368 & \\
\hline$\alpha-S y n$ wt & $2.16 \mathrm{E}+07$ & $1.94 \mathrm{E}+06$ \\
\hline$\alpha-S y n 2 K R$ & $2.07 E+07$ & $1.76 \mathrm{E}+06$ \\
\hline$\alpha-S y n$ AA & $2.36 \mathrm{E}+07$ & $4.95 E+06$ \\
\hline $\mathbf{n}$ & 6 & \\
\hline t-test (wt/2KR) & 0.55184 & \\
\hline t-test (wt/2AA) & 0.17285 & \\
\hline$\alpha-S y n$ wt & $3.33 \mathrm{E}+07$ & $3.78 \mathrm{E}+06$ \\
\hline$\alpha$-Syn wt/VPS4dn co-transfection & $2.50 \mathrm{E}+07$ & $5.12 \mathrm{E}+06$ \\
\hline $\mathbf{n}$ & 7 & \\
\hline t-test & 0.000054 & \\
\hline$\alpha-S y n-S U M O-2$ & $5.10 \mathrm{E}+07$ & $1.40 \mathrm{E}+05$ \\
\hline$\alpha-S y n-S U M O-2$ /VPS4dn & $4.07 E+07$ & $5.35 E+06$ \\
\hline $\mathbf{n}$ & 8 & \\
\hline t-test & 0.00281561 & \\
\hline YFP-APPsw & $3.30 \mathrm{E}+07$ & $3.50 \mathrm{E}+05$ \\
\hline YFP-APPsw-SUMO-2 & $3.85 \mathrm{E}+07$ & $2.96 \mathrm{E}+05$ \\
\hline n & 8 & \\
\hline t-test & 0.104734285 & \\
\hline YFP-APPsw/SUMO-2-wt & $4.60 \mathrm{E}+07$ & $3.47 E+06$ \\
\hline YFP-APPsw/SUMO-2-DGG & $4.02 \mathrm{E}+07$ & $1.82 \mathrm{E}+06$ \\
\hline $\mathbf{n}$ & 8 & \\
\hline t-test & 0.092198482 & \\
\hline
\end{tabular}




\section{Curriculum Vitae}

Marcel Kunadt

born on March $4^{\text {th }} 1984$ in Eisenhüttenstadt, Germany

Sültebecksbreite 1

37075 Göttingen, Germany

Phone: +49176 23420179

E-Mail: mkunadt@gwdg.de

\section{Education}

07/2011 - present

Georg-August Universität Göttingen, Germany

$\mathrm{PhD}$ Thesis "Extracellular vesicle release of $\alpha$-Synuclein is mediated by SUMOylation" in the Department of Psychiatry, University Medical Center Göttingen under supervision of Prof. Dr. Anja Schneider

07/2011 - present

Georg-August Universität Göttingen, Germany

GGNB PhD Program "Molecular Physiology of the Brain"

$10 / 2004-10 / 2010$

Georg-August Universität Göttingen, Germany

Institute of Microbiology and Genetics

Dept. Genetics of Eukaryotic, diploma degree in biology

$07 / 2000-07 / 2003$

OSZ Gottfried-Wilhelm Leibniz Eisenhüttenstadt, Germany Abitur degree (general qualification for university entrance) 


\section{Publications}

M. Kunadt, K. Eckermann, A. Stuendl, J. Gong, B. Russo, K. Strauss, S. Rai, S. Kügler, L. Falomir Lockhart, M. Schwalbe, P. Krumova, L. M. Oliveira, M. Bähr, W. Möbius, J. Levin, A. Giese, N. Kruse, B. Mollenhauer, R. Geiss-Friedländer, A. C. Ludolph, A. Freischmidt, M. S. Feiler, K. M. Danzer, M. Zweckstetter, T. M. Jovin, M. Simons, J. H. Weishaupt, A. Schneider, Extracellular vesicle sorting of $\alpha$-Synuclein is regulated by sumoylation. Acta neuropathologica, DOI 10.1007/s00401-015-1408-1, (2015). 